\title{
Estimativas das coordenadas de comprimento para um espaço de papel furado
}

\author{
Minoru Enrique Akiyama Figueroa \\ DisSERTAÇÃO/TESE APRESENTADA \\ $\mathrm{AO}$ \\ Instituto de Matemática e EstatísticA \\ DA \\ Universidade DE SÃo PAUlo \\ PARA \\ OBTENÇÃO DO TÍTULO \\ $\mathrm{DE}$ \\ Mestre/DOUTOR EM CiênCIAS \\ Programa: Doutorado em Matemática Aplicada \\ Orientador: Prof. Dr. André Salles de Carvalho
}

Durante o desenvolvimento deste trabalho o autor recebeu auxílio financeiro da CNPq

São Paulo, abril de 2018 
Estimativas das coordenadas de comprimento para um espaço de papel furado

Esta é a versão original da dissertação/tese elaborada pelo candidato (Minoru Enrique Akiyama Figueroa), tal como submetida à Comissão Julgadora. 


\section{Estimativas das coordenadas de comprimento para um espaço de papel furado}

\footnotetext{
Esta versão da dissertação/tese contém as correções e alterações sugeridas pela Comissão Julgadora durante a defesa da versão original do trabalho, realizada em 05/06/2018. Uma cópia da versão original está disponível no Instituto de Matemática e Estatística da Universidade de São Paulo.
}

Comissão Julgadora:

- Prof. Dr. André Salles de Carvalho (orientador) - IME-USP

- Prof. Dr. Sylvain Bonnot - IME-USP

- Prof. Dr. Salvador Addas Zanata - IME-USP

- Prof. Dr. Marcel Vinhas Bertolini - UFPA

- Prof. Dr. Zbigniew Nitecki - Tufts University 


\section{Agradecimentos}

Quiero agradecer en primer lugar a Adriana, por todo su amor, su apoyo y cariño conmigo, durante el tiempo que hemos estado junto y en particular durante el tiempo que hemos estado en São Paulo.

A mis padres María Helena Figueroa y Tamotsu Akiyama, a mi hermana Harumi Akiyama y mi cuñado Julien Lambert, a mis suegros Luz Elena Salcedo y Domingo Araujo, por su amor y por todo el apoyo que me han dado.

A todas las personas que he conocido aquí en São Paulo y que de una u otra manera me han ayudado durante estos años. En especial a Mirko, Catarina, Diego, Fernando, Segundo, Leticia, Eduardo, João, Felipe, Sylvain, Marcel, Raibel, Germán, Arcelino, Gerard, entre muchos otros.

Quiero agradecer de manera especial a mi orientador André Salle de Carvalho, por ser una gran persona que durante mi doctorado siempre estuvo dispuesto a enseñarme, aconsejarme y en general a ayudarme de cualquier forma y sin ninguna duda. 


\section{Resumo}

SOBRENOME, A. B. C. Estimativas das coordenadas de comprimento para um espaço de papel furado. 2018. 120 f. Tese (Doutorado) - Instituto de Matemática e Estatística, Universidade de São Paulo, São Paulo, 2018.

Espaços de papel são quocientes métricos obtidos ao identificar num multipolígono $P \subset \mathbb{C}$, pares de subsegmentos do bordo de $P$ por isometrías que revertem orientação. Tais espaços aparecem por exemplo em [dC05], onde mostra-se uma receita para construir, a partir de certos homeomorfismos definidos numa superfície compacta, um homeomorfismo chamado de pseudo-Anosov generalizado (pois generalizam os pseudo-Anosov clássicos definidos por Thurston), o qual está definido sobre um espaço de papel homeomorfo à superfície original. Por outro lado, um espaço de papel $S$ tem uma estrutura complexa natural em todos seus pontos exceto, possívelmente, num conjunto fechado $\mathcal{Q} \subset S$ de pontos chamados irregulares. Neste trabalho será considerado a superfície hiperbólica $S_{\infty}$ obtida a partir de uma esfera de papel $S$ com $m$ pontos irregulares, ao tirar dela seu conjunto de pontos irregulares junto com os pontos com curvatura positiva. Na esfera de papel furada $S_{\infty}$ será descrita uma família $\Gamma_{\infty}$ de curvas geodésicas que descompõem à $S_{\infty}$ em calças e serão calculados estimativas para os comprimentos hiperbólicos dessas curvas.

Palavras-chave: Superfícies gaita de fole, espaços de papel, pontos cônicos, pontos irregulares. 


\section{Abstract}

SOBRENOME, A. B. C. Estimates of the length coordinates for a punctured paper space. 2018. 120 f. Tese (Doutorado) - Instituto de Matemática e Estatística, Universidade de São Paulo, São Paulo, 2018.

Paper spaces are metric quotients obtained by identifying, given a multipolygon $P \subset \mathbb{C}$, pairs of subsegments of the edge of $P$ by orientation reversing isometries. Such spaces appear for example in [dC05], where a recipe is given to construct, starting from certain homeomorphism on a compact surface, an homeomorphism called generalized pseudo-Anosov map (as these generalize the classical pseudo-Anosov defined by Thurston), which is defined on a paper space homeomorphic to the original surface. On the other hand a paper space $S$ has a natural complex structure at each of its points except, possibly, on a closed set $\mathcal{Q} \subset S$ of points called irregular. In this work will be considerer the hyperbolic surface $S_{\infty}$ obtained, from a paper sphere $S$ with $m$ irregularities, by taking from it the set of irregular points together with the positive curvature points. On the punctured paper sphere $S_{\infty}$ will be described a family of geodesic curves $\Gamma_{\infty}$ which decompose $S_{\infty}$ into pairs of pants and will be also calculated stimates for the hyperbolic lengths of those curves.

Keywords: Bagpipes surfaces, paper spaces, conics points, irregular points. 


\section{Sumário}

1 Preliminares $\quad 5$

1.1 Espaços métricos . . . . . . . . . . . . . . . . . . . . . . . 5

1.2 Grupos Fuchsianos . . . . . . . . . . . . . . . . . . . 10

1.3 Curvas geodésicas em superfícies hiperbólicas . . . . . . . . . . . . . . . . 20

1.4 Superfícies flauta e superfícies gaita de fole . . . . . . . . . . . . 26

2 Espaços de papel $\quad 33$

2.1 Ferradura justa . . . . . . . . . . . . . . . . . . . . 33

2.2 Espaços de papel . . . . . . . . . . . . . . . . . . . . . . . . . . . . . . . . . . . .

2.3 Esferas de papel . . . . . . . . . . . . . . . . . . . . . . . . . 43

2.4 Pontos irregulares isolados . . . . . . . . . . . . . . . . . 48

3 Esfera de papel furada $\quad \mathbf{5 5}$

3.1 Decomposição de $S_{\infty} \ldots \ldots \ldots \ldots \ldots \ldots \ldots \ldots \ldots$

3.2 Modelo base . . . . . . . . . . . . . . . . . . . . 57

3.3 Estimativas superiores das coordenadas de comprimento . . . . . . . . . . . . 65

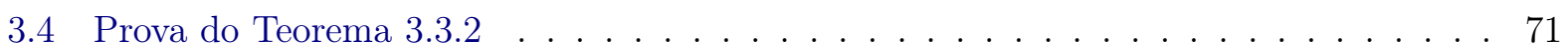

3.5 Estimativas inferiores das coordenadas de comprimento . . . . . . . . . . . . 78

3.6 A ferradura justa furada . . . . . . . . . . . . . . . . . 84

$\begin{array}{ll}\text { Referências Bibliográficas } & 89\end{array}$ 


\section{Introdução}

Este trabalho teve como origem uma pergunta em principio simple e um pouco inocente: Se numa esfera você tira uma sequência infinita de pontos especiais em algum sentido, que convergem à outro ponto especial, considera na nova superfície a métrica hiperbólica e olha para a sequência de geodésicas fechadas simples que separam um furo do seguinte, Qual é o comportamento asintótico dos comprimentos dessas geodésicas à medida que as curvas se "aproximam" ao limite da sequência dos pontos? Ficam eles limitados superiormente/inferiormente? Crescem para infinito? e nesse caso, Qual sería sua taxa de crescimento? Naturalmente a primeira pergunta, feita dessa forma, resulta muito vaga e pouco interessante. Assim, vou a precisar explicar com um pouco mais de detalhes os atores envolvidos e a motivação por trás dessa questão.

Em primeiro lugar a esfera a que me refiro não é uma esfera qualquer; é uma esfera de papel. Espaços de papel são quocientes obtidos ao identificar adequadamente pares de subsegmentos da fronteira de um polígono plano ou, mais geralmente de um multipolígono plano, de maneira que o espaço resultante seja, depois de tirar dele possívelmente alguns que outros pontos, uma superfície orientável com uma estrutura cônico-plana. Logo uma esfera de papel é um espaço de papel homeomorfo a uma esfera. Voltando ao caso geral dos espaços de papel, existem muitos exemplos deles na matemática, sendo o exemplo mais conhecido o caso do toro como quociente de um quadrado ao identificar pares de lados opostos; de maneira mais geral qualquer superfície fechada de gênero $g \geq 1$ pode ser realizada (Topológicamente) como um espaço de papel a partir de um $4 g$-ágono regular, colando pares de seus lados adequadamente. Outros exemplos de espaços de papel de grande importancia, em particular no estudo da teoría de bilhares, são as chamadas de superfície de translação, ver [Zor06], [Ran16].

Porém os exemplos que interessan principalmente nesta tese estão relacionados com uma classe especial de aplicações sobre superfícies chamados de homeomorfismos pseudo-Anosov generalizados, ver [dC05], [dCH10]. A definição formal de um homeomorfismo pseudo-Anosov generalizado será dada no començo do capítulo 2, embora estos não serão estudados com detalhes neste trabalho. Por enquanto posso antecipar que a diferença entre um pseudo-Anosov generalizado e um pseudoAnosov normal é que o primeiro admite na suas folheações invariantes uma quantidade infinita de singularidades, só que estas acumulan-se num número finito de pontos. Uma maneira de construir homeomorfismos pseudo-Anosov generalizados consiste em usar um train track generalizado invariante por um certo endomorfismo e logo, usando a matríz de transição associada ao train track generalizado, construir a partir de um número finito de retângulo cujos tamanhos são determinados por informação vindo dessa matríz, um espaço de papel cujas identificações são também determinados pelo train track generalizado. A ação do endomorfismo sobre o train track generalizado extende-se agora sobre o espaço de papel num homeomorfismo que é pseudo-Anosov generalizado e que será um pseudo-Anosov comum no caso que o train track tenha finitas arestas. Este método inspirado claramente no artigo clássico de Bestvina-Handel, ver [BH95], no qual serve para construir homeomorfismos pseudo-Anosov também sobre espaços de papel, foi desenvolvido por A. Salles de Carvalho em [dC05]. Aquí, o espaço de papel além de ser homeomorfo a uma superfície de gênero finito, possui uma estrutura complexa natural definido em todos seus pontos fora do conjunto de pontos irregulares, isto é, do conjunto de pontos de acumulação das singularidades do homeomorfismo pseudo-Anosov generalizado associado, mas sem embargo neste caso, esta estrutura complexa extende-se de maneira única a cada um destos pontos irregulares. As folheações mensuradas trans- 
versais do homeomorfismo correspondem à projeção das folheações verticais e horizontais naturais dos retângulos usados na construção antes (e muito vagamente) descritas, e correspondem a sua vez com as trajetórias verticais e horizontais de uma diferencial quadrática integrável cujo número de polos, todos de ordem um, são infinitos e são acumulados exatamente no conjunto de pontos irregulares.

Voltando à pergunta inicial feita ao começo desta introdução, esta tese começou analisando a esfera de papel associada a um homeomorfismo pseudo-Anosov generalizado particular chamado de ferradura justa, o qual têm infinitos 1-singularidades acumulando-se num único ponto fixo de tal homeomorfismo. A idéia foi tirar desta esfera de papel tanto o ponto irregular como os pontos 1singulares das folheações transversais e olhar para o comportamento das geodésicas nessa superfície a medida que estas separam cada vez mais o furo associado ao ponto irregular dos furos associados à 1-singularidades que na esfera de papel estão menos próximo do ponto irregular. A idéia também consistiu em começar com este exemplo para logo fazer o mesmo análise com uma família infinita de esferas de papel descritas em [dCH10], associadas a uma família de endomorfismos unimodais definidos sobre um intervalo da reta real. No artigo mencionado pode-se ver três possívels tipos de esferas de papel: O primeiro tipo só tem um ponto irregular e o conjunto de 1-singularidades conforman uma única órbita homoclínica desse ponto irregular; o segundo tipo têm $k+1$ pontos irregulares, sendo o conjunto de pontos 1-singulares também uma única órbita, só que ésta converge no pasado a um dos pontos irregulares e no futuro acumula-se nos outros $k$ pontos irregulares, os quais conforman uma órbita periódica do homeomorfismo pseudo-Anosov generalizado; o tercer tipo não tem pontos irregulares, logo o homeomorfismo na esfera de papel corresponde a um homeomorfismo pseudo-Anosov mesmo. Sendo o objetivo do trabalho analisar os casos de esferas de papel furadas com infinitos furos, o tercer tipo acima mencionado não era de interés. Com respeito aos outros dos tipos a evidência conhecida no momento fizeram pensar que aconteceriam dois fenómenos diferentes em cada um dos tipo: No segundo tipo, que os comprimentos das curvas geodésicas ficariam uniformemente limitadas e, no primeiro tipo, que os comprimentos das geodésicas cresceriam para infinito; neste caso, interesaba saber qual era a taxa de crescimento. Porém depois de estudar a esfera de papel furada associada a ferradura justa (a qual corresponde a um papel do primeiro tipo na família unimodal), resultou que em ambos tipos de espaços de papel os comprimentos das curvas geodésicas ficam limitadas por constantes positivas.

Boa parte desta tese pretende explicar como se chegou a este resultado. De fato o abordagem da tese virou a um estudo puramente geométrico das superfícies hiperbólicas obtidas ao tirar de uma esfera de papel qualquer seus pontos irregulares e pontos 1-cônicos; a definição de tais pontos encontra-se no capítulo 2 da tese, porém no caso de esferas de papel associadas a pseudo-Anosov generalizados, os pontos 1-cônicos correspondem as 1-singularidades das folheações transversais invariantes pelo homeomorfismo. A única hipótese imposta no espaço de papel foi que o número de pontos irregulares seja sempre finito, isto para ter presente os espaços de papel associados aos Pseudo-Anosov generalizados. Uma vez fixada uma esfera de papel com finitas irregularidades e uma vez furada esta esfera, foi descrita uma decomposição em calças desta superfície e foram estimados os comprimentos das geodésicas que realizam tal decomposição.

Aquí é importante destacar que a pergunta sobre o comportamento asintótico dos comprimentos das geodésicas feita ao começo não é tão inocente como se pode perceber inicialmente. A razão disto é a seguinte: Você pode ter a esfera de papel descrita em detalhe, tirar os respectivos pontos irregulares e 1-cônicos dela, a superfície resultante é claramente uma superfície hiperbólica e em consequência será biholomorfa a um quociente do tipo $\mathbb{H} / G$, onde $G$ é um grupo Fuchsiano, você até pode construir as curvas fechadas homotópicas as geodésicas que decompõem à esfera furada em calças. Mas daí a conhecer quais são exatamente as geodésicas da decomposição e conhecer quais são seus comprimentos hiperbólicos é outra história. Para isso é preciso conhecer com exactitude o grupo Fuchsiano $G$ ou a função de uniformização $\mathbb{H} \longrightarrow \mathbb{H} / G$. Aínda para o caso da esfera de Riemann menos um número finito de pontos isto não é um problema trivial, ver por exemplo [Hem], [ZT88]. Além disso, no caso de uma esfera de papel que seja biholomorfa à esfera de Riemann, a métrica 
conhecida da esfera de papel e a métrica esférica da esfera de Riemann não são necessariamente comparáveis, com o qual também não pode-se fazer úso de estimativas conhecidas da métrica de Poincaré num dominio do plano complexo. $\mathrm{O}$ argumento usado nesta tese para fazer as estimativas das geodésicas está explicado no Lema 3.2.2; aínda o argumento seja bem simple, este depende de uma boa escolha de uma densidade Boreliana definido sobre um anel conveniente, assim que a parte difícil foi conseguir a densidade Boreliana certa de maneira que com ela, das estimativas de comprimento das geodésicas calculadas, pode-se extrair informação substancial. Acredito que a técnica desenvolvida para conseguir tais estimativas superiores dos comprimentos de geodésicas numa esfera de papel furada com finitas irregularidades sirva também para qualquer espaço de papel furado com finitos ou infinitos pontos irregulares.

$\mathrm{Na}$ procura de material bibliográfico que me ajuda-se com alguma idéia de como clacular os comprimentos de geodésicas numa esfera de papel furada, achei um artigo de A. Basmajian cujos desenhos se ajustaram à superfície associada à ferradura justa furada. As superfícies flauta estudadas no artigo [Bas93] a que me refiro, foi de muita ajuda para o entendimento da geometría envolvida nas esferas de papel furadas desta tese, aínda não forem de muita ajuda para o cálculo das estimativas procuradas. Para o estudo das esferas de papel furadas com um número finito qualquer de pontos irregulares foi necessário generalizar um pouco o conceito de superfície flauta desse artigo; assim nascem as superfícies gaita de fole as quais são definidas formalmente no final do capítulo 1 deste trabalho. Tanto as flautas como as gaitas de fole resultam muito úteis no estudo de propriedades e, na procura de contra-exemplos, na geometría de superfícies do tipo infinito respeito à teoría das superfícies hiperbólicas do tipo finito, em especial, em relação a teoría de Teichmüller neste tipo de superfícies. Convido ao leitor a revisar os artigos [Shi03], [ALP $\left.{ }^{+} 11\right]$, [ALPS12b], [ALPS12a], [APLS11], [ALPS16], [BŠ17], [Sar15], [Kin11] e [Evr17], entre outros, onde são estudados diferentes aspectos relativos a teoría de Teichmüller em superfícies do tipo infinito; aínda nesta teses a teoría de Teichmüller é apenas rasgunhada, é importante ressaltar que na maioria dos artigos referidos uma característica desejável numa superfície do tipo infinito consiste em que exista uma decomposição em calças de uma superfície, onde os comprimentos da multicurva que realiza tal decomposição sejam uniformemente limitado por acima. Um dos objetivos deste trabalho tem que ver com conseguir condições para garantir a existencia de uma multicurva numa esfera de papel furada cujos comprimentos sejam limitados por uma constante positiva.

Este trabalho está dividido da seguinte forma. O capítulo 1, como seu nome o indica, é um capítulo de preliminares. Nele são apresentados alguns conceitos e resultados básicos na literatura especializada que serão usádos em maior ou menor medida no desenvolvimento da tese. Na seção 1.4 é apresentado o conceito de superfície flauta de A. Basmajian e algumas propriedades, para imediatamente depois introduzir o conceito de superfície gaita de fole e generalizar nelas propriedades válidas nas superfícies flauta. Aínda as superfícies gaita de fole sejam uma generalização natural das superfícies flautas, no meu conhecimento não existia uma definição formal delas nem um estudo sistemático das mesmas. Entre os resultados originais neste capítulo, além da definição de superfície gaita de fole, estão o Teorema 1.4.5 o qual garante a boa definição de gaita de fole, e o Teorema 1.4.6 o qual determina se a estrutura Fuchsiana de uma gaita de fole é um grupo Fuchsiano do primeiro ou de segundo tipo, dependendo se são satisfeitas certas hipóteses. Também está a Proposição 1.4.11 que caracteriza as superfícies gaita de fole em função do conjunto dos fins num dominio da esfera de Riemann.

No capítulo 2 é apresentado o conceito de espaço de papel. Este capítulo podería ser considerado parte dos preliminares da tese pois tanto o conceito de espaço de papel como as propriedades deles mostradas no capítulo forem desenvolvidos em diferentes artigos como por exemplo [dCH12], [dCH10], [dCH13], [Ber12] e [dC05]. Porém, sendo os espaços de papel um protagonista principal desta tese, colocou-se a teoría dedicada aos espaços de papel como um capítulo aparte e forem feitos as demostrações de algumas das suas propriedades para fazer o texto o mais auto-contído possível. Na seção 2.4 sim são apresentados dois resultados originais que são necessarios para o desenvolvimento do capítulo 3. O primeiro destos resultados, a Proposição 2.4.2, caracteriza os 
pontos irregulares num espaço de papel que não são pontos de acumulação de pontos cônicos; este resultado será importante pois, sob as suposições impostas sobre as esferas de papel do capítulo 3, permite descartar que esse fenómeno acontece. O segundo resultado, o Teorema 2.4.4, mostra que numa superfície de papel, um ponto irregular isolado não somente é ponto de acumulação de pontos cônicos, se não que em particular será ponto de acumulação de pontos 1-cônicos.

O capítulo 3 contém os princiapais resultados desta tese. Primeiramente serão identificadas as esferas de papel furadas com as superfícies gaita de fole justas definidas no final do capítulo 1, e será descrita uma decomposição relativamente natural desta superfície em calças. A seguir serão estimados por acima os comprimentos das curvas geodésicas da decomposição em calças num modelo simple de esfera de papel furada que foi chamado de modelo base. Os argumentos usádos nas estimativas feitas no modelo base serão generalizadas para obter um método que permite calcular estimativas superiores dos comprimentos de todas exceto um número finito das geodésicas na decomposição em calças de uma esfera de papel furada qualquer. Este resultado, o Teorema 3.3.2, será o resultado principal de toda a tese; com ele pode-se calcular de maneira relativamente fácil as estimativas superiores dos comprimentos a partir de informação local na cicatriz de uma esfera de papel furada perto de um ponto irregular. A Proposição 3.3.5 será uma conseqüência imediata do Teorema 3.3.2 e do Teorema 1.4.6; este criterio vai determinar sob certas hipóteses se a estrutura Fuchsiana de uma esfera de papel furada é do primeiro tipo. Que a estrutura Fuchsiana de uma superfície seja do primeiro tipo é uma caracterísitica desejável no caso particular de esferas de papel associadas a um homeomorfismo pseudo-Anosov generalizado pois, na procura de uma possível generalização do Teorema de hiperbolização de Thurston para estos homeomorfismos, isto facilitaria de maneiras diferentes o úso do Teorema de uniformização simultanea de Bers (Ver [Ota96], [Ber16] e [J.H06], Proposição 6.12.4, para mais detalhes). Na seção 3.5 consegue-se estimativas inferiores para os comprimentos das geodésicas na esfera furada do modelo base, ver Proposição 3.5.1. Nesta mesma seção consegue-se também uma estimativa superior para o comprimento de uma geodésica fechadas simple que separa dois furos escolhidos convenientemente do resto dos furos numa esfera de papel furada arbitraria; tal estimativa (Ver Proposição 3.5.4) permite a sua vez estimar por baixo os comprimentos das geodésicas que decompoem à uma esfera furada em calças. Achei apropriado finalizar com um análisis do espaço de papel furado associado a ferradura justa, considerando que foi com este exemplo particular que a maioria das idéias desenvolvida na tese forem realizadas; assim na última seção são aplicados os resultados do capítulo 3 para conseguir estimativas dos comprimentos de diferentes geodésicas nesta superfície flauta. 


\section{Capítulo 1}

\section{Preliminares}

Neste capítulo de preliminares serão apresentados algumas noções básicas necessárias em sua maioria para o desenvolvimento dos seguintes capítulos. O leitor pode se quiser pasar este capítulo e voltar a ele quando considere necessário. Algumas das referencias principais usadas são as seguintes: Para a seção 1.1 o livro [Iva01], para as seções 1.2, 1.3 e 1.4, [Kat88], [Bus92], [Bon09], [Mas88], [J.H06], [YT92], [Bas93] e [FM11].

\subsection{Espaços métricos}

Definição 1.1.1. Uma métrica em um conjunto $X$ é uma função $d_{X}: X \times X \longrightarrow \mathbb{R} \cup\{\infty\}$ que satisfaz as seguintes propriedades: Para quaisquer $x, y, z \in X$,

i) Não-negatividade: $d_{X}(x, y) \geq 0$;

ii) Simetría: $d_{X}(x, y)=d_{X}(y, x)$;

iii) Desigualdade triangular: $d_{X}(x, z) \leq d_{X}(x, y)+d_{X}(y, z)$;

iv) Identidade de indiscerníveis: $x=y$ se, e somente se, $d_{X}(x, y)=0$.

Se a propriedade iv) é sustituída pela seguinte:

iv') $x=y \Rightarrow d_{X}(x, y)=0$;

dize-se que $d_{X}$ é uma semi-métrica.

O par $\left(X, d_{X}\right)$ é dito um espaço métrico ou semi-métrico respectivamente. Se $x, y \in X$, o valor $d_{X}(x, y)$ é chamado de distância entre $x$ e $y$. Toda métrica (semi-métrica) em $X$ determina uma topología no mesmo conjunto, a qual é gerada pela coleção dos conjunto chamados de bolas abertas $B_{X}(x, r):=\left\{y \in X / d_{X}(x, y)<r\right\}, x \in X, r>0$. Se $d_{1}$ e $d_{2}$ são duas métricas no mesmo conjunto $X$, dize-se que $d_{1}$ e $d_{2}$ são topológicamente equivalentes se a topología induzida por $d_{1}$ e a topología induzida por $d_{2}$ são as mesmas.

Seja $\left(X, d_{X}\right)$ um espaço métrico, $x \in X$ e $A, B \subset X$ não-vazíos. A distância entre $x$ e $A$ é o valor:

$$
d_{X}(x, A):=\inf _{a \in A} d_{X}(x, a)
$$

A distância entre os conjuntos $A$ e $B$ é o valor:

$$
d_{X}(A, B):=\inf _{\substack{a \in A \\ b \in B}} d_{X}(a, b)
$$

O diámetro de $A$ é o valor: 


$$
\operatorname{diam}_{X}(A):=\sup _{x, y \in A} d_{X}(x, y)
$$

Se $r>0, B_{X}(A, r), \bar{B}_{X}(A, r)$ e $\mathbb{S}_{X}(A, r)$ denotam os conjuntos:

$$
\begin{aligned}
& B_{X}(A, r):=\left\{x \in X / d_{X}(x, A)<r\right\} \\
& \bar{B}_{X}(A, r):=\left\{x \in X / d_{X}(x, A) \leq r\right\} \\
& \mathbb{S}_{X}(A, r):=\left\{x \in X / d_{X}(x, A)=r\right\}
\end{aligned}
$$

As vezes será escrito $B_{\left(X, d_{X}\right)}(A, r)$ ou $B_{d_{X}}(A, r)$ para denotar a bola aberta $B_{X}(A, r)$ quando seja conveniente resaltar a métrica do espaço $X$; a mesma notação será adotada com bolas fechadas e esferas de centro $A$ e raio $r$ respectivamente. Por outro lado, para simplificar um pouco a notação, será escrito a métrica do espaço $X$ como $d$ em vez de $d_{X}$ se não há perigo de confução.

Definição 1.1.2. Uma aplicação $f:\left(X, d_{X}\right) \longrightarrow\left(Y, d_{Y}\right)$ entre espaços métricos dize-se que preserva distância, se para todo $x_{1}, x_{2} \in X, d_{Y}\left(f\left(x_{1}\right), f\left(x_{2}\right)\right)=d_{X}\left(x_{1}, x_{2}\right)$. Se além disso $f$ é sobrejetóra (logo bijetóra), dize-se que $f$ é uma isometría. Dize-se que $f$ é uma isometría local, se para todo $x \in X$ existe $r_{x}>0$ tal que a restrição $f: B_{d_{X}}\left(x, r_{x}\right) \longrightarrow B_{d_{Y}}\left(f(x), r_{x}\right)$ é uma isometría.

Definição 1.1.3. Dado um espaço métrico $(X, d)$ qualquer, um caminho em $X$ é uma aplicação continua $\gamma:[a, b] \longrightarrow X$. Seu comprimento é o valor:

$$
|\gamma|_{X}:=\sup \sum_{i} d\left(\gamma\left(t_{i}\right), \gamma\left(t_{i+1}\right)\right) \in \mathbb{R} \cup\{\infty\},
$$

onde o supremo é tomado sobre todas as partições $a=t_{0}<t_{1}<t_{2}<\ldots<t_{N}=b$ do intervalo $[a, b]$. Dize-se que o caminho $\gamma$ é retificável se $|\gamma|_{X}<\infty$. A métrica intrínseca induzida por $d$ é a função $\widehat{d}: X \times X \longrightarrow \mathbb{R} \cup\{\infty\}$ definida, para todo $x, y \in X$, por:

$$
\widehat{d}(x, y):=\inf \left\{|\gamma|_{X} / \gamma \text { é um caminho em } X \text { conectando } x \text { e } y\right\}
$$

Observação 1.1.4. i) É fácil ver que a função $\widehat{d}$ satisfaz as propriedades da definição 1.1.1. No caso que não exista um caminho conectando dois pontos $x, y \in X$, tem-se $\widehat{d}(x, y)=\infty$. Esta é a razão de permitir na definição de métrica que a distância entre dois pontos estem a uma distância infinita.

ii) Em geral as métricas $\widehat{d}$ e $d$ não são topológicamente equivalentes (ver [Iva01], exercício 2.3.9.), mas a topología induzida por $\widehat{d}$ sempre é mais fina que a induzida pela m étrica $d$; em particular ambas topologías coincidem se $(X, d)$ é um espaço métrico compacto. Por outro lado, se $\gamma$ é um caminho em $X$, é facil provar que o comprimento de $\gamma$ não depende de qual das duas métricas, $d$ ou $\widehat{d}$, é usada; logo a métrica intrínseca induzida por $\widehat{d}$ é a mesma métrica $\widehat{d}$.

Definição 1.1.5. Uma métrica $d$ em $X$ dize-se que é intrínseca, e que $(X, d)$ é um espaço de comprimento, se $d=\widehat{d}$. Dize-se que a métrica $d$ é estritamente intrínseca se ela é intrínseca e o infimo na definição de $\widehat{d}$ for realizado, é dizer, dados $x, y \in X$, existe um caminho $\gamma$ conectando $x$ e $y$ tal que $d(x, y)=|\gamma|_{X}$.

Proposição 1.1.6. Toda métrica intrínseca compacta é estritamente intrínseca. 
Seja $X$ um conjunto qualquer e $\mathcal{G}$ uma sub-coleção de subconjuntos de $X$. Dados $x, y \in X$, dize-se que $x$ e $y$ são identificados por $\mathcal{G}$ se $x=y$ ou existe $g \in \mathcal{G}$ tal que $x, y \in g$. Assím a coleção $\mathcal{G}$ determina em $X$ uma relação $R_{\mathcal{G}}$ refléxiva, simétrica, más não necessariamente transitiva; será transitiva se, e somente se, os elementos de $\mathcal{G}$ são dois a dois disjuntos: nesse caso a relação $R_{\mathcal{G}}$ é de equivalência e dize-se que $\mathcal{G}$ é uma partição. Em geral, se $R$ é uma relação de equivalência em $X$, o conjunto quociente $X / R$ é a coleção de $R$-clases de equivalência $R[x]:=\{y \in X / x R y\}$, $x \in X$, a qual determina uma partição de $X$; a aplicação $\pi_{R}: X \longrightarrow X / R$, dada por $x \longmapsto R[x]$, chama-se de $R$-projeção ou simplemente de projeção, se não houver perigo de confusão. Se $R$ é uma relação reflexiva e simétrica em $X$, um subconjunto $U$ de $X$ dize-se que é $R$-saturado se todo ponto $R$-relacionado com algum ponto de $U$ deve estar em $U$.

Definição 1.1.7. Considere-se um espaço métrico $(X, d)$ e uma relação $R$ em $X$ reflexiva e simétrica. Dados $x, y \in X$, Um $R$-itinerario de $x$ à $y$ é uma sequência finita $\left(\left(p_{i}, q_{i}\right)\right)_{i=0}^{k}$ em $X^{2}$ tal que $x R p_{0}, q_{i} R p_{i+1}$, para cada $i=0,1, \ldots, k-1$, e $q_{k} R y$. Seu $d$-custo define-se como a soma:

$$
L^{R}\left(\left(p_{i}, q_{i}\right)_{i=0}^{k}\right):=\sum_{i=0}^{k} d\left(p_{i}, q_{i}\right)
$$

A ideia aquí é que, para moverse do ponto $x$ ao ponto $y$ vía um $R$-itinerário $\left(\left(p_{i}, q_{i}\right)\right)_{i=0}^{k}$, cada passo de $p_{i}$ à $q_{i}$ tem custo $d\left(p_{i}, q_{i}\right)$, más o passo de $q_{i}$ à $p_{i+1}$, sendo ambos pontos $R$-relacionados, é gratuito.

A função $d^{R}(x, y): X \times X \longrightarrow \mathbb{R} \cup\{\infty\}$, definida para cada $x, y \in X$, por

$$
d^{R}(x, y):=\inf \left\{L^{R}\left(\left(p_{i}, q_{i}\right)\right) /\left(\left(p_{i}, q_{i}\right)\right) \text { é um } R \text {-itinerario de } x \text { a } y\right\}
$$

é uma semi-métrica em $X$ a qual, a sua vez, determina uma relação de equivalência $\sim_{R}$ no mesmo $X$, onde dois pontos são $\sim_{R}$-relacionados se a $d^{R}$-semidistância entre eles é 0 . A semi-métrica $d^{R}$ em $X$ projeta-se naturalmente numa métrica (a qual será denotada também por $d^{R}$ ) no conjunto quociente $X / \sim_{R}$.

Definição 1.1.8. Se $(X, d)$ é um espaço métrico e $R$ é uma relação em $X$ reflexiva e simétrica, o quociente métrico de $X$ por $R$ define-se como o espaço métrico $\left(X / \sim_{R}, d^{R}\right)$. Por simplicidade, será denotado $X / d^{R}$ a tal quociente métrico.

Observação 1.1.9. $\quad$ i) Se a relação em $X$ é induzida por uma coleção $\mathcal{G}$ de subconjuntos de $X$, chama-se a um $R_{\mathcal{G}}$-itinerário entre dois pontos de $\mathcal{G}$-itinerário, e usa-se a notação $d^{\mathcal{G}}$ e $\sim_{\mathcal{G}} \mathrm{em}$ vez de $d^{R_{\mathcal{G}}}$ e $\sim_{R_{\mathcal{G}}}$ respectivamente.

ii) Se $R$ é uma relação de equivalência, o quociente métrico $X / d^{R}$ e o conjunto quociente $X / R$ não necessariamente coincidem como conjunto. Por exemplo se $X=\mathbb{R}$ e $\mathcal{G}=\{\mathbb{Q}, \mathbb{R} \backslash \mathbb{Q}\}, \mathbb{R} / \mathcal{G}$ consiste em dois pontos enquanto que $X / d^{\mathcal{G}}$ só tem um ponto. Por outro lado, comparando o quociente métrico $X / d^{R}$ com o quociente toplógico $X / \sim_{R}$, a topología do segundo é mais fina que a topología do quociente métrico: Se $\pi_{d^{R}}: X \longrightarrow X / d^{R}$ denota a projeção sobre o quociente métrico, então ela é continua pois $d^{R}\left(\pi_{d^{R}}(x), \pi_{d^{R}}(y)\right)=d^{R}(x, y) \leq d(x, y)$; sendo $\pi_{d^{R}}=i d \circ \pi_{\sim_{R}}$, tem-se que $i d: X / \sim_{R} \longrightarrow X / d^{R}$ é continua. A seguinte proposição é consecüência imediata desta última observação.

Proposição 1.1.10. Se $(X, d)$ é um espaço métrico compacto e $R$ é uma relação em $X$ reflexiva e simétrica, o quociente métrico $X / d^{R}$ e o quociente topológico $X / \sim_{R}$ são homeomorfos. 
Referente ao quociente métrico de um espaço de comprimento tem-se o seguinte resultado, ver [Iva01], página 62-63.

Proposição 1.1.11. Se $(X, d)$ é um espaço de comprimento e $R$ é uma relação em $X$ reflexiva e simétrica, o quociente métrico $\left(X / d^{R}, d^{R}\right)$ é um espaço de comprimento.

A continuação vai ser enunciado um teorema devido a R. L. Moore, que dá condições suficientes para que o quociente topológico de uma 2-esfera seja novamente uma 2-esfera. Antes disso, algumas definições.

Definição 1.1.12. Uma separação de um espaço topológico $X$ é uma decomposição de $X$ como união disjunta $X=A \sqcup B$ onde $A$ e $B$ são subconjuntos fechados e não vazíos de $X$. Dize-se que $X$ é conexo se não existe uma separação de $X$. Um subconjunto $C \subset X$ separa dois pontos $x, y \in X$ (dois subconjuntos $D, E \subset X)$ se existir uma separação $X \backslash C=A \sqcup B$, com $x \in A$ e $y \in B(D \subset A$ e $E \subset B$ ). Se $X$ é conexo e $X \backslash C$ não é, dize-se que $C$ separa $X$. Um continuum é um espaço de Hausdorff compacto e conexo.

Definição 1.1.13. Uma relação de equivalência $R$ num espaço topológico $X$ dize-se fechada, se $R$ é fechado como subconjunto no espaço produto $X \times X$. Uma decomposição $\mathcal{G}$ de $X$ é semi-continua superiormente se cada $g \in \mathcal{G}$ é compacto e a relação $R_{\mathcal{G}}$ é fechada; e é monótona se cada $g \in \mathcal{G}$ for conexo.

Observação 1.1.14. No caso que $X$ seja um espaço métrico com métrica $d$, uma relação de equivalência $R$ em $X$ é fechada se, e somente se, $x_{n} \longrightarrow x, y_{n} \longrightarrow y$ e $\left(x_{n}, y_{n}\right) \in R$ implica que $(x, y) \in R$.

Teorema 1.1.15 (Moore, [Moo25]). O quociente topológico da 2-esfera por uma decomposição monótona e semi-continua superiormente cujos elementos não a separam é homeomorfa a 2-esfera.

Definição 1.1.16. Um espaço métrico é um dendrito se é um continuum localmente conexo que não contém nenhuma curva fechada simples. Um dendrito local é um continuum tal que cada ponto tem uma vizinhança fechada a qual é um dendrito.

A seguinte proposição mostra algumas propriedades básicas dos dendritos, ver [Why63].

Proposição 1.1.17. Se G é um dendrito, então:

i) Quaisquer dois pontos de $G$ são separados por um tercer ponto de G. recíprocamente, um continuum com essa propriedade é um dendrito.

ii) Se $x, y \in G, x \neq y$, existe um único arco em $G$ os conectando.

iii) $G$ é contrátil.

iv) Todo sub-continuum de $G$ é um dendrito.

v) Todo subconjunto conexo de $G$ é conexo por caminhos, e a interseção de quaisquer dois conexos em $G$ é conexa. 
Um ponto $p$ em um continuum $G$ chama-se de extremidade se possui vizinhanças arbitrariamente pequenha cuja fronteira é um único ponto. O ponto $p \in G$ chama-se de ponto de corte, se $\{p\}$ separa $G$.

Proposição 1.1.18. Um continuum $G$ é um dendrito se, e somente se, todos seus pontos são ou extremidades ou pontos de corte.

Definição 1.1.19. Uma coleção $\mathcal{G}$ de subconjuntos de um espaço topológico $X$ é não-separada, se nenhum elemento de $\mathcal{G}$ separa dois pontos pertenecentes a cada um dos outros elementos de $\mathcal{G}$. Dize-se que $\mathcal{G}$ é dendrítica se é não-separada e, dado $g \in \mathcal{G}$ e $y \in g$ existe $g^{\prime} \in \mathcal{G}$ que separa $g$ de $y$.

Teorema 1.1.20 (ver [Why63]). Uma decomposição dendrítica $\mathcal{G}$ de um continuum $X$ é semicontinua superiormente e o quociente topológico $X / \mathcal{G}$ é um dendrito.

Para finalizar a seção, serám definidos os conceitos de cone métrico e superfície cônico-plana, o qual servirá para determinar uma estrutura comforme nos espaços de papel no próximo capítulo.

Definição 1.1.21. Considere-se um espaço métrico $(X, d)$. Define-se o cone métrico sobre $(X, d)$, denotado por $\left(\operatorname{Cone}(X), d_{c}\right)$, como o quociente de $[0,+\infty) \times X$ pela relação de equivalência que identifica num mesmo ponto todos os pontos da forma $(0, x), x \in X$, e deixa os outros intactos, dotado da métrica $d_{c}: \operatorname{Cone}(X) \times \operatorname{Cone}(X) \longrightarrow \mathbb{R}$ definida, para cada classe $[s, x],[t, y] \in \operatorname{Cone}(X)$, por:

$$
d_{c}([s, x],[t, y]):=\left\{\begin{array}{ccc}
\sqrt{s^{2}+t^{2}-2 s t \cos (d(x, y))} & , \text { se } & d(x, y) \leq \pi \\
s+t & , \text { se } & d(x, y) \geq \pi
\end{array}\right.
$$

Observação 1.1.22. $\quad$ i) A função $d_{c}$ acima definida é em efeito uma métrica sobre o conjunto cone $(X)$; além disso, se $d$ é uma métrica estritamente intrínseca no espaço $X$, é possivel provar que $d_{c}$ também é estritamente intrínseca em Cone $(X)$, ver [Iva01], Teorema 3.6.1.

ii) Para todo $x, y \in X,[0, x]=[0, y]$, pelo qual será denotado por $[0]$ à classe de equivalência $[0, x]$; este ponto será chamado de ápice do cone. É imediato da definição de $d_{c}$ que, para todo $[t, x] \in \operatorname{Cone}(X), d_{c}([0],[t, x])=t$. Isto implica que para $r>0, B_{d_{c}}([0], r)=\{[t, x] / t<r\}$.

Seja $\mathbb{S}_{\xi}$ o círculo de raio $\xi>0$, dotado da métrica intrínseca $d_{\mathbb{S}_{\xi}}$ e considere-se o cone métrico sobre $\mathbb{S}_{\xi}$, o qual denotaremos por simplicidade por Cone $(\xi)$ em vez de Cone $\left(\mathbb{S}_{\xi}\right)$. Para todo $\xi$, o cone métrico Cone $(\xi)$ é homeomorfo ao plano complexo $\mathbb{C}$ : Tome o homeomorfismo $\phi_{\xi}$ : Cone $(\xi) \rightarrow \mathbb{C}$, $\phi_{\xi}\left(\left[t, \xi e^{i \theta}\right]\right):=t^{1 / \xi} e^{i \theta}$. Por outro lado, é fácil provar que os cones Cone $\left(\xi_{1}\right)$ e Cone $\left(\xi_{2}\right)$ são isométricos se, e somente se, $\xi_{1}=\xi_{2}$; sendo $\phi_{1}$ além de um homeomorfismo uma isometría entre $\mathbb{C}$ e Cone(1), o anterior implica que Cone $(\xi)$ nunca é isometrico ao plano quando $\xi \neq 1$. A função $\psi_{\xi}$ : Cone $(\xi) \rightarrow \mathbb{C}$ dada por $\psi_{\xi}\left(\left[t, \xi e^{i \theta}\right]\right):=t e^{i \xi \theta}$ é uma isometría local em todos os pontos do cone diferente do ápice. Em efeito, dado $t_{0}>0$ e $\theta_{0} \in \mathbb{R}$, considere-se $0<r<\min \left\{\frac{\pi}{2}, \frac{\pi}{2 \xi}\right\}$ e a vizinhança $U\left(\theta_{0}, r\right)$ do $\left[t_{0}, \xi e^{i \theta_{0}}\right]$ das classes $\left[t, \xi e^{i \theta}\right]$ tais que $t>0$ e $\left|\theta-\theta_{0}\right|<r$ (observe-se que $r$ foi escolhido suficientemente pequenho de forma que $d_{\mathbb{S}_{\xi}}\left(\xi e^{i \theta_{1}}, \xi e^{i \theta_{2}}\right)=\xi \cdot\left|\theta_{1}-\theta_{2}\right|<\pi$, para todo $\theta_{1}, \theta_{2} \in\left(\theta_{0}-r, \theta_{0}+r\right)$.). Para todo $x_{1}, x_{2} \in U\left(\theta_{o}, r\right)$, suponha que $x_{j}=\left[t_{j}, \xi e^{i \theta_{j}}\right]$, onde $\left|\theta_{j}-\theta_{0}\right|<r$, para $j=1,2, \log$, 


$$
\begin{aligned}
\left|\psi_{\xi}\left(x_{1}\right)-\psi_{\xi}\left(x_{2}\right)\right| & =\left|t_{1} e^{i \xi \theta_{1}}-t_{2} e^{i \xi \theta_{2}}\right| \\
& =\left(t_{1}^{2}+t_{2}^{2}-2 \operatorname{Re}\left(t_{1} \cdot t_{2} e^{i \xi\left(\theta_{1}-\theta_{2}\right)}\right)\right)^{1 / 2} \\
& =\left(t_{1}^{2}+t_{2}^{2}-2 t_{1} \cdot t_{2} \cos \left(\xi\left(\theta_{1}-\theta_{2}\right)\right)\right)^{1 / 2} \\
& =d_{c}\left(x_{1}, x_{2}\right) .
\end{aligned}
$$

Assim $\psi_{\xi}$ é uma isometría quando é restrito ao aberto $U\left(\theta_{0}, r\right)$. Naturalmente o homeomorfismo $\phi_{\xi}$ determina uma estrutura complexa no cone métrico Cone $(\xi)$; o que é interesante aquí é que as cartas locais complexas $\left(U(\theta, r), \psi_{\xi}\right)$, com $\theta \in \mathbb{R}$ e $r>0$ suficientemente pequenho são compatíveis com tal estrutura pois, para todo $z=\rho e^{i \theta} \in \mathbb{C}^{*}:=\mathbb{C} \backslash\{0\}$,

$$
\psi_{\xi} \circ \phi_{\xi}^{-1}(z)=\psi_{\xi}\left(\left[\rho^{\xi}, \xi e^{i \theta}\right]\right)=\rho^{\xi} e^{i \theta \xi}=z^{\xi}
$$

Definição 1.1.23. Uma superfície cônico-plana é um espaço métrico $(X, d)$ tal que, para cada $x \in X$, existem $\xi, r>0$ tais que a bola $B_{X}(x, r)$ é isométrica à $B_{\text {Cone }(\xi)}([0], r)$.

Denote-se por $\mathcal{V}$ o conjunto de pontos em uma superfície cônico-plana $(X, d)$ que satisfazem a seguinte propriedade: $x \in \mathcal{V}$ se existe uma isometría $f: B_{X}(x, r) \rightarrow B_{\text {Cone }(\xi)}([0], r)$ tal que $f(x)=[0]$, para algum $r>0$ e $\xi \neq 1$. Tais pontos são chamados de pontos cônicos de $X$ enquanto que os pontos de $X \backslash \mathcal{V}$ são chamados de pontos planares de $X$. Observe-se que pelas propriedades dos cones métricos sobre um círculo, o conjunto de pontos cônicos $\mathcal{V}$ é discreto pois qualquer ponto perto de $x \in \mathcal{V}$ diferente de $x$ tem uma vizinhança localmete isométricoa um aberto de $\mathbb{C}$, logo deve ser planar. Daí é fácil concluir que toda superfície cônico-plana admite uma estrutura complexa natural, a qual é localmente plana no subespaço $X \backslash \mathcal{V}$.

\subsection{Grupos Fuchsianos}

Nesta tese será considerado o modelo do semiplano superior $\mathbb{H}=\{z \in \mathbb{C} / \operatorname{Im} z>0\}$ como modelo do plano hiperbólico, equipado com a métrica Riemanniana de curvatura constante igual à $-1, \lambda_{\mathbb{H}}(z)|d z|$ onde,

$$
\lambda_{\mathbb{H}}(z)|d z|:=\frac{|d z|}{\operatorname{Im} z}
$$

Assim, se $\gamma:[a, b] \longrightarrow \mathbb{H}, \gamma(t)=(x(t), y(t))$, é uma curva parametrizada diferenciável por pedaços, o comprimento hiperbólico de $\gamma$ é o valor,

$$
\ell_{\mathbb{H}}(\gamma):=\int_{\gamma} \frac{|d z|}{\operatorname{Im} z}=\int_{a}^{b} \frac{\sqrt{\left(x^{\prime}(t)\right)^{2}+\left(y^{\prime}(t)\right)^{2}}}{y(t)} d t
$$

Dados $z_{1}, z_{2} \in \mathbb{H}$, a distância hiperbólica entre estos pontos define-se como,

$$
d_{\mathbb{H}}\left(z_{1}, z_{2}\right):=\inf _{\gamma} \ell_{\mathbb{H}}(\gamma)
$$

onde o ínfimo é tomado sobre todas as curvas $\gamma \subset \mathbb{H}$ diferenciável por pedaços que conectam ambos pontos. O bordo no infinito de $\mathbb{H}$, denotado por $\partial_{\infty} \mathbb{H}$, é a fronteira de $\mathbb{H}$ como subespaço da esfera de Riemann $\hat{\mathbb{C}}=\mathbb{C} \cup\{\infty\}$. Assim $\partial_{\infty} \mathbb{H}$ é a reta real extendida $\hat{\mathbb{R}}=\mathbb{R} \cup\{\infty\} ; \overline{\mathbb{H}}$ denotará a união $\mathbb{H} \cup \partial_{\infty} \mathbb{H}$ com a topología de subespaço de $\widehat{\mathbb{C}}$. É importante resaltar que a topología em $\mathbb{H}$ induzida 
pela distância $d_{\mathbb{H}}$ coincide com a topología de $\mathbb{H}$ como subespaço da esfera de Riemann, porém ambos espaços não são métricamente equivalentes pois $\left(\mathbb{H}, d_{\mathbb{H}}\right)$ é completo em quanto que $\left(\mathbb{H}, d_{\widehat{\mathbb{C}}}\right)$ não é.

Definição 1.2.1. Um arco geodésico em $\mathbb{H}$ é uma curva $\gamma:(a, b) \longrightarrow \mathbb{H}$ que realiza a distância hiperbólica entre os pontos que conecta. Uma linha geodésica ou simplemente uma geodésica em $\mathbb{H}$, é um arco geodésico que não pode ser extendido a um arco geodésico maior.

Dados dois pontos $z_{1}, z_{2} \in \mathbb{H}$ existe um único arco geodésico com extremidades estos pontos: tal arco será denotado por $\left[z_{1}, z_{2}\right]$. As geodésicas em $\mathbb{H}$ são semicírculos euclideanos que intersectam ortogonalmente o círculo no infinito $\hat{\mathbb{R}}$; igualmente, dados $a_{1}, a_{2} \in \hat{\mathbb{R}}$ diferentes, existe uma única geodésica com extremidades no infinito $a_{1}$ e $a_{2}$, a qual será denotada por $\left[a_{1}, a_{2}\right]$, aínda suas extremidades não são parte de $\mathbb{H}$ (Observe-se que $[a, \infty], a \in \mathbb{R}$, é uma semireta ortogonal à $\mathbb{R}$ cuja base é $a)$. Um conjunto $X \subset \overline{\mathbb{H}}$ dize-se que é convexo se para todo $z_{1}, z_{2} \in X$, tem-se $\left[z_{1}, z_{2}\right] \subset X$.

Definição 1.2.2. Uma sequência aninhada de geodésicas é uma sequência $\left\{L_{n}\right\}_{n \geq 0}$ de linhas geodésicas em $\mathbb{H}$ mutuamente disjuntas em $\overline{\mathbb{H}}$ tais que, para cada $n \geq 1, L_{n-1}$ e $L_{n+1}$ estão em diferentes componentes de $\mathbb{H} \backslash L_{n}$. Dize-se que a sequência aninhada converge à geodésica $L$, se as extremidades de $L_{n}$ convergem as extremidades de $L$, como sequência em $\hat{\mathbb{R}}$. Se em vez disso as extremidades de $L_{n}$ convergem a um ponto, dize-se que a sequência diverge ao bordo de $\mathbb{H}$.

Observação 1.2.3. As vezes será considerado o primeiro elemento de uma sequência aninhada de geodésicas seja um horocíclo como na figura 1.1.

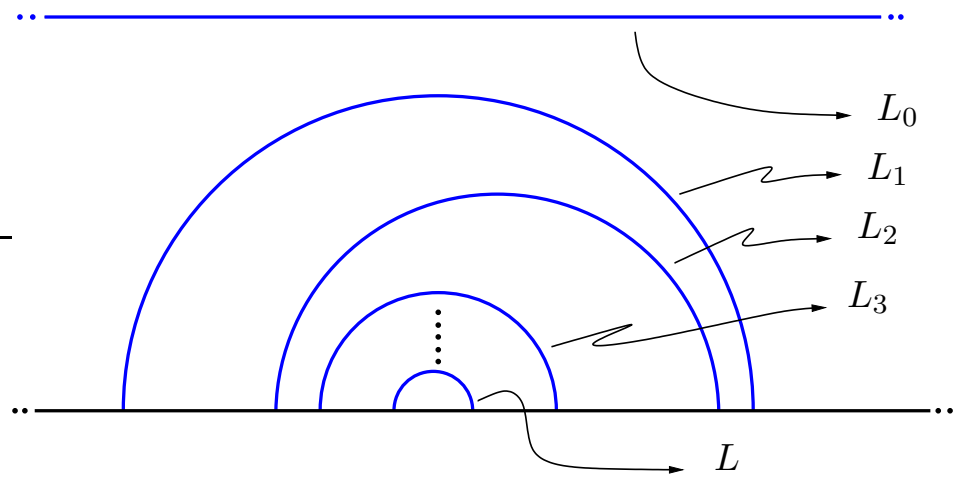

Figura 1.1: Uma sequência aninhada de geodésicas que converge à geodésica $L$

Proposição 1.2.4 (ver [Bas93]). Se uma sequência aninhada de geodésicas $\left\{L_{n}\right\}_{n \geq 0}$ converge à geodésica $L$, então

$$
\sum_{n \geq 0} d_{\mathbb{H}}\left(L_{n}, L_{n+1}\right)<+\infty
$$

Suponha que as geodésicas de uma sequência aninhada $\left\{L_{n}\right\}_{n \geq 0}$. estão orientadas de maneira que $L_{n+1}$ fica na direita de $L_{n}$, para $n \geq 0$. Dado qualquer $n \geq 0$, existe um único arco geodésico $O_{n}$ ortogonal à $L_{n}$ e $L_{n+1}$ que conecta ambas curvas; denote-se por $z_{n}^{-}$o ponto de interseção de $L_{n}$ com $O_{n-1}$, e por $z_{n}^{+}$o ponto de interseção de $L_{n}$ com $O_{n}$, ver figura 1.2. Considere-se os números reais $s_{n}, n \geq 1$, definidos por $s_{n}:=\epsilon_{n} \cdot d_{\mathbb{H}}\left(z_{n}^{-}, z_{n}^{+}\right)$, onde $\epsilon_{n}=+1$ se $z_{n}^{-}$está na esquerda de $z_{n}^{+}$e $\epsilon_{n}=-1$ se $z_{n}^{-}$está na direita de $z_{n}^{+}$, isto relativo à orientação da geodésica $L_{n}$. A sequência $\left(s_{n}\right)_{n \geq 1}$ será chamada de coordenadas de torção da sequência aninhada $\left\{L_{n}\right\}_{n \geq 0}$. 


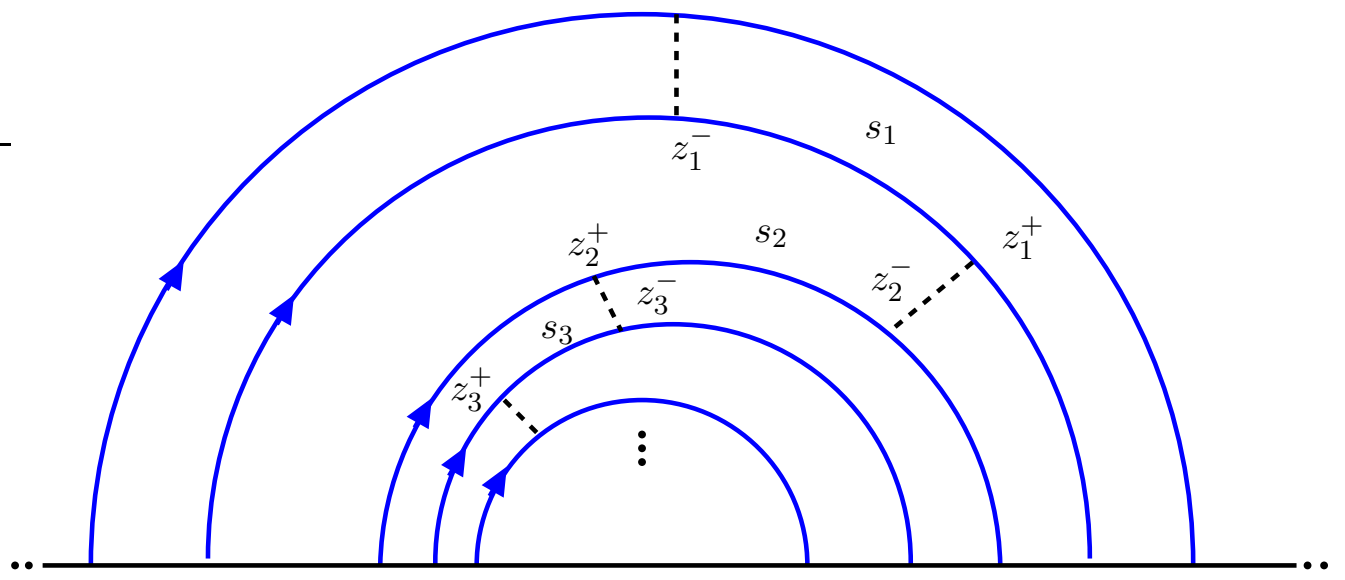

Figura 1.2: Torção de uma sequência de geodésica. Observe-se que no desenho $s_{1}>0, s_{2}<0, s_{3}<0$.

Proposição 1.2.5 (ver [Bas93]). Seja $\left\{L_{n}\right\}_{n \geq 0}$ uma sequência aninhada de geodésicas com coordenadas de torsão $\left(s_{n}\right)_{n \geq 1}$. A sequência $\left\{L_{n}\right\}_{n \geq 0}$ converge a uma geodésica se,

$$
\sum_{n \geq 0} d_{\mathbb{H}}\left(L_{n}, L_{n+1}\right)<+\infty \quad \text { e } \sum_{n \geq 1}\left|s_{n}\right|<+\infty
$$

Denote-se por $\operatorname{Isom}^{+}(\mathbb{H})$ ao grupo de isometría de $\mathbb{H}$ que preservam orientação. $\operatorname{Isom}^{+}(\mathbb{H})$ consiste nas transformações lineares fraccionarias $z \mapsto \frac{a z+b}{c z+d}$ tais que $a, b, c, d \in \mathbb{R}$ e $a d-b c=1$. Assim, identifica-se este grupo com $\mathrm{PSL}_{2}(\mathbb{R}):=S L_{2}(\mathbb{R}) /\{ \pm \mathrm{Id}\}$.

Definição 1.2.6. Seja $f \in \operatorname{Isom}^{+}(\mathbb{H})$ diferente da identidade e suponha que $A \in S L_{2}(\mathbb{R})$ representa à $f$. Dize-se que $f$ é:

i) Parabólico, se $|\operatorname{Tra} A|=2$

i) Hiperbólico, se $|\operatorname{Tra} A|>2$

i) Elítico, se $|\operatorname{Tra} A|<2$

Observação 1.2.7. Lembre-se que toda transformação linear fraccionaria diferente da identidade tem no máximo dois pontos fixos. Os elementos parabólicos correspondem as isometrías com um único ponto fixo o qual está em $\hat{\mathbb{R}}$; éstos são conjugados com a transformação $z \mapsto z-1$ ou $z \mapsto z+1$. Um elemento $f$ é hiperbólico quando tem dois pontos fixos em $\hat{\mathbb{R}}$. Neste caso a geodésica com extremidades os pontos fixos de $f$ chama-se o eixo de $f$ e será denotado por $\mathcal{E}_{f}$ : O eixo de $f$ é a única geodésica invariante pela ação de $f$. É fácil ver que um elemento hiperbólico é conjugado em $P S L_{2}(R)$ a uma transformação $z \mapsto \lambda \cdot z$, para algum $\lambda>1$. Os elementos elíticos são os únicos que tem um ponto fixo em $\mathbb{H}$ (O seu segundo ponto fixo está no complemento de $\overline{\mathbb{H}})$; eles são conjugados a transformações da forma $z \mapsto e^{i \theta} \cdot z, \operatorname{com} \theta \in \mathbb{R} \backslash 2 \pi \mathbb{Z}$. A figura 1.3 mostra os pontos fixos de cada uma das classes de isometrías anteriores e a ação delas sobre $\overline{\mathbb{H}}$.

Definição 1.2.8. Um grupo Fuchsiano é um subgrupo discreto de $\mathrm{PSL}_{2}(\mathbb{R})$.

Proposição 1.2.9. $G<\mathrm{PSL}_{2}(\mathbb{R})$ é Fuchsiano se, e somente se, $G$ age descontinuamente em $\mathbb{H}$, isto é, para todo $z \in \mathbb{H}$, existe uma vizinhança $U$ de $z$ tal que o conjunto $\{g \in G / g \cdot U \cap U \neq \emptyset\}$ é finito. 


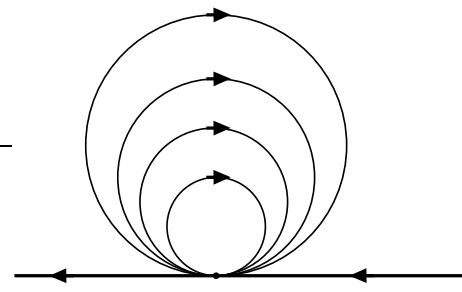

Parabólico

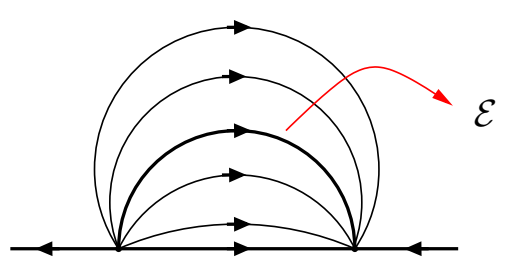

Hiperbólico

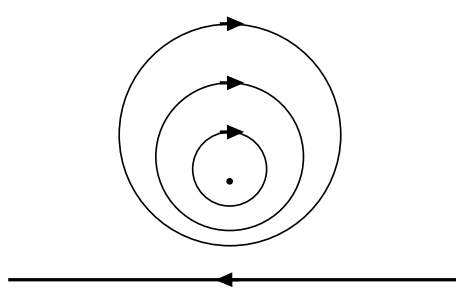

Elítico

Figura 1.3: Isometrías do tipo parabólico, hiperbólico e elítico respectivamente.

Na proposição acima, basta mostrar que $G$ age descontinuamente em algum $z \in \mathbb{H}$ para concluir que este grupo age descontinuamente em cada ponto de $\mathbb{H}$, ver [Kat88]. Como consequência da proposição acima, dado um grupo Fuchsiano $G$ e um ponto $z \in \mathbb{H}$, a $G$-órbita de $z, G z:=\{g(z) / g \in$ $G\}$ não pode acumular-se em nenhum ponto de $\mathbb{H}$. Porém, se $G$ é infinito, a $G$-órbita de um ponto de $\mathbb{H}$ como subespaço em $\hat{\mathbb{C}}$ deve ter pelo menos um ponto de acumulação em $\hat{\mathbb{C}}$; sendo $\mathbb{H}$ invariante por $G$, os pontos de acumulação de $G z$ estão contidos no bordo $\hat{\mathbb{R}}$, para qualquer $z \in \mathbb{H}$. É fácil ver que o conjunto de pontos limite das $G$-órbitas de pontos de $\mathbb{H}$ é o mesmo indiferentemente do ponto de $\mathbb{H}$ escolhido, pelo qual a notação na seguinte definição tem sentido.

Definição 1.2.10. Seja $G$ um grupo Fuchsiano. O conjunto limite de $G$, denotado por $\Lambda_{G}$, consiste nos pontos de acumulação (como subespaço de $\hat{\mathbb{C}}$ ) da órbita $G z$, para algum $z \in \mathbb{H}$.

Proposição 1.2.11. Se $G<\mathrm{PSL}_{2}(\mathbb{R})$ é um grupo Fuchsiano, o conjunto limite $\Lambda_{G}$ é $G$-invariante. Além disso, uma das seguintes três possibilidades é satisfeita:

i) $\Lambda_{G}$ tem máximo dois pontos.

ii) $\Lambda_{G}=\hat{\mathbb{R}}$.

iii) $\Lambda_{G}$ é um conjunto de Cantor.

Quando $\Lambda_{G}$ tem 0,1 ou 2 pontos somente, é dizer, no caso $i$ ) da proposição acima, dize-se que o grupo é elementar. Todo grupo Fuchsiano elementar, ou é um grupo cíclico ou é conjugado com o grupo gerado pelas transformações $z \mapsto \lambda \cdot z, \lambda>1$, e $z \mapsto-\frac{1}{z}$, ver [Bea83], Teorema 8.1.2. Em particular um grupo Fuchsiano elementar e sem elementos elíticos é cíclico infinito. Nesta tese serão considerados somente grupos Fuchsianos sem elementos elíticos e, salvo em alguns casos específicos, grupos não-elementares. Observe-se que $G$ não tem elementos elíticos se, e somente se, $G$ é livre de torção, é dizer, se $g^{n}=\mathrm{Id}$ para $g \in G$ e $n \geq 1$, então $g=\mathrm{Id}$. Quando o grupo Fuchsiano é nãoelementar, éste deve conter uma família infinita de elementos hiperbólicos nenhum dos quais tem um ponto fixo común. Cabe resaltar também que todo grupo Fuchsiano não-elementar é não-Abeliano.

Definição 1.2.12. Um grupo Fuchsiano não elementar $G$ chama-se do primeiro tipo se $\Lambda_{G}=\hat{\mathbb{R}}$ e chama-se do segundo tipo se $\Lambda_{G}$ é um conjunto de Cantor.

Suponha que $G$ é um grupo Fuchsiano do segundo tipo, então $\hat{\mathbb{R}} \backslash \Lambda_{G}$ é a união disjunta de uma coleção enumerável de intervalos abertos do círculo no infinito; cada um destes intervalos será chamado de intervalo de descontinuidade de G. O semi-plano aberto limitado por um intervalo de descontinuidade e pela geodésica cujas extremidades são as extremidades desse intervalo será chamado de semi-espaço de bordo de $G$. Se existe um elemento $g \in G$ cujo eixo limita um semiespaço de bordo de $G$, dize-se que $g$ é um elemento hiperbólico de bordo de $G$. Dado $X \subset \overline{\mathbb{H}}$ e $G$ um subgrupo de $\mathrm{PSL}_{2}(\mathbb{R})$, denota-se por $S_{t a}(X)$ ao estabilizador de $X$ relativo à ação de $G$, isto é, $\operatorname{Sta}_{G}(X):=\{g \in G / g X=X\}$. 
Definição 1.2.13. Seja $X \subset \overline{\mathbb{H}}$ um conjunto não-vazío, $G$ um grupo Fuchsiano e $G_{0}$ um subgrupo de $G$. Dize-se que $X$ é precisamente invariante pelo $G_{0}$ em $G$ se,

i) $G_{0}=S t a_{G}(X)$.

ii) $g X \cap X=\emptyset$, se $g \in G \backslash G_{0}$.

Proposição 1.2.14 (Ver [Mas88], pagina 106). Seja $G$ um grupo Fuchsiano do segundo tipo e $I \subset \hat{\mathbb{R}}$ um intervalo de descontinuidade de $G$. Denote-se por $L$ a linha geodésica que limita o intervalo I $e$ por $H$ o semiplano limitado por $I \cup L$. Então $G_{0}=\operatorname{Sta}_{G}(I)=\operatorname{Sta}_{G}(L)=S t a_{G}(H)$ é trivial ou é um grupo cíclico gerado por um elemento hiperbólico de $G$. Além disso, $I, L$ e $H$ são precisamente invariantes pelo $G_{0}$ em $G$.

O seguinte lema resulta útil para determinar se um grupo Fuchsiano é do segundo tipo; observese que este é quase um recíproco da proposição anterior.

Lema 1.2.15. Seja $G$ um grupo Fuchsiano não-elementar e $I \subset \widehat{\mathbb{R}}$ um intervalo aberto. Se $I$ é precisamente invariante por $\operatorname{Sta}_{G}(I)$ em $G$, então $G$ é um grupo Fuchsiano do segundo tipo e I está contido num intervalo de descontinuidade de $G$.

Demonstração. Basta provar que $I \cap \Lambda_{G}=\emptyset$. Suponha por redução ao absurdo que existe $x \in$ $I \cap \Lambda_{G}=\emptyset$. Não há perda de generalidade em supor que $x$ é um ponto fixo de um elemento hiperbólico $g \in G$, ver Corolario 2.3.5 em [J.H06]. $g \notin S t a_{G}(I)$ pois nesse caso fixaria, além do ponto $x$, as extremidades de $I$, logo sería a identidade. Sendo $I$ precisamente invariante por $\operatorname{Sta}_{G}(I)$, tem-se $g I \cap I=\emptyset$ o qual é uma contradição pois $x=g(x) \in I \cap g(I)$.

Observe-se que na proposição acima, se $S t a_{G}(I)$ é um subgrupo não trivial, então o intervalo $I$ será um intervalo de descontinuidade do grupo $G$ pois nesse caso qualquer elemento em $\operatorname{Sta}_{G}(I)$ diferente da identidade deve fixar as extremidades de $I$, logo será um elemento de bordo de $G$ e as extremidades de $I$ pertencem ao conjunto limite de $G$. Se em cambio $\operatorname{Sta}_{G}(I)$ é trivial, pode acontecer que $I$ seja um subconjunto proprio de um intervalos de descontinuidade de $G$.

Definição 1.2.16. Considere-se um grupo Fuchsiano não-elementar $G$. A região de Nielsen de $G$, denotado por $\mathcal{N}(G)$, define-se como o menor subespaço de $\mathbb{H}$ não-vazío, fechado, convexo e $G$-invariante.

Observação 1.2.17. $\quad$ i) Se $G$ é do primeiro tipo claramente $\mathcal{N}(G)=\mathbb{H}$. Se $G$ é do segundo tipo e $\left\{H_{n}\right\}_{n}$ denota a coleção dos semiplanos de bordo de $G$, então $\mathcal{N}(G)=\mathbb{H} \backslash \cup_{n} H_{n}$. Observe-se que $\mathcal{N}(G)$ é o menor convexo em $\mathbb{H}$ que contém todas as linhas geodésicas cujas extremidades estão em $\Lambda_{G}$.

ii) Suponha que $G$ é um grupo Fuchsiano e $G_{0}$ um subgrupo de $G$ (logo também Fuchsiano), então $\Lambda_{G_{0}} \subset \Lambda_{G}$ com o qual $\mathcal{N}\left(G_{0}\right) \subset \mathcal{N}(G)$.

Se $G$ é um subgrupo de $\mathrm{PSL}_{2}(\mathbb{R})$ e $X, Y \subset \overline{\mathbb{H}}$, dize-se que $X$ e $Y$ são $G$-conjugados se os estabilizadores de $X$ e de $Y$ relativos à $G$ são $G$-conjugados, é dizer, se existe $g \in G$ tal que $g \cdot \operatorname{Sta}_{G}(X) \cdot g^{-1}=\operatorname{Sta}_{G}(Y)$. Um elemento $g \in G$ é primitivo em $G$, se não existe $h \in G$ e $n \geq 2$ tal que $h^{n}=g$. 
Proposição 1.2.18 (Ver [Mas88]). Sejam $G_{1}$ e $G_{2}$ dois grupos Fuchsianos, $g_{0} \in G_{1} \cap G_{2}$ um elemento hiperbólico primitivo de bordo tanto para $G_{1}$ como para $G_{2}$, tais que,

$$
\mathcal{N}\left(G_{1}\right) \cap \mathcal{N}\left(G_{2}\right)=\mathcal{E}_{g_{0}}
$$

então,

i) $\left\langle G_{1}, G_{2}\right\rangle$ é um grupo Fuchsiano.

ii) $\mathcal{N}\left(G_{i}\right)$ é precisamente invariante por $G_{i}$ em $\left\langle G_{1}, G_{2}\right\rangle$, para $i=1,2$.

iii) Se $I \subset \hat{\mathbb{R}}$ é um intervalo de descontinuidade de $G_{2}$ o qual não é $G_{2}$-conjugado ao intervalo de descontinuidade de $G_{2}$ limitado por $\mathcal{E}\left(g_{0}\right)$, então $I$ é um intervalo de descontinuidade de $\left\langle G_{1}, G_{2}\right\rangle$.

Demonstração. Pode-se assumir, depois de fazer uma conjugação, que o eixo de $g_{0}$ coincide com a geodésica $[0, \infty]$. Suponha que $\mathcal{N}\left(G_{1}\right)$ está na componente "esquerda"e $\mathcal{N}\left(G_{2}\right)$ está na componente "direita"de $\mathbb{H} \backslash \mathcal{E}_{g_{0}}$, como mostra a figura 1.4. Sejam $I_{1}$ e $I_{2}$ os intervalos de descontinuidade de $G_{1}$ e $G_{2}$ respectivamente limitados por $\mathcal{E}_{g_{0}}$, isto é $I_{1}=[0,+\infty]$ e $I_{2}=[-\infty, 0]$ (Como subconjuntos de $\hat{\mathbb{R}}$ ); denote-se por $H_{1}$ e $H_{2}$ os respectivos semiplanos de bordo limitados por $I_{1} \cup \mathcal{E}_{g_{0}}$ e $I_{2} \cup \mathcal{E}_{g_{0}}$, logo $\mathcal{N}\left(G_{1}\right) \subset H_{2} \cup \mathcal{E}_{g_{0}}, \mathcal{N}\left(G_{2}\right) \subset H_{1} \cup \mathcal{E}_{g_{0}}$. Observe-se que $\left\langle g_{0}\right\rangle=S t a_{G_{i}}\left(I_{i}\right)$, para $i=1,2$.

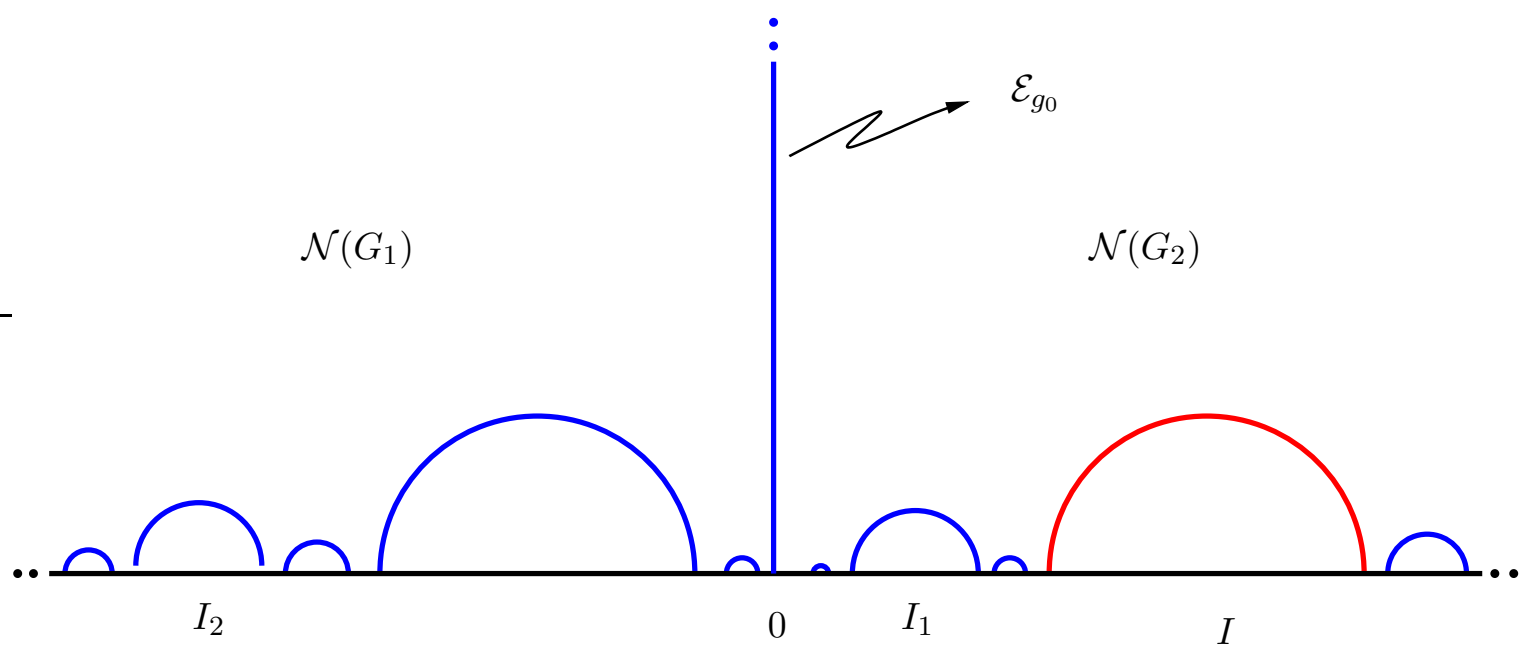

Figura 1.4: $O$ eixo $\mathcal{E}_{g_{0}}$ e alguns dos semiespaços de $\mathcal{N}\left(G_{1}\right)$ e $\mathcal{N}\left(G_{2}\right)$

Mostraremos primeiro que $\mathcal{N}\left(G_{1}\right)$ é precisamente invariante por $G_{1}$ em $\left\langle G_{1}, G_{2}\right\rangle$. Pela definição da região de Nielsen, $g \mathcal{N}\left(G_{1}\right)=\mathcal{N}\left(G_{1}\right)$, quando $g \in G_{1}$, logo basta mostrar que para todo $g \in$ $G_{2} \backslash G_{1}, g \mathcal{N}\left(G_{1}\right) \cap \mathcal{N}\left(G_{1}\right)=\emptyset$., para concluir que $\mathcal{N}\left(G_{1}\right)$ é precisamente invariante por $G_{1}$ em $\left\langle G_{1}, G_{2}\right\rangle$. Em efeito, se $g \in G_{2} \backslash G_{1}$, em particular $g \in\left\langle g_{0}\right\rangle=S t a_{G_{2}}\left(I_{2}\right)$, logo pela Proposição 1.2.14, $g H_{2} \cap H_{2}=\emptyset$ e $g \mathcal{E}_{g_{0}} \cap \mathcal{E}_{g_{0}}=\emptyset$. Como $\mathcal{N}\left(G_{1}\right) \subset H_{2} \cup \mathcal{E}_{g_{0}}$, então $g \mathcal{N}\left(G_{1}\right) \cap \mathcal{N}\left(G_{1}\right)=\emptyset$. Similarmente, prova-se que $\mathcal{N}\left(G_{2}\right)$ é precisamente invariante por $G_{2}$ em $\left\langle G_{1}, G_{2}\right\rangle$. Isto termina a prova de $\left.i i\right)$.

Considere-se $z$ um ponto interior de $\mathcal{N}\left(G_{1}\right)$. Sendo $G_{1}$ um grupo Fuchsiano, existe uma vizinhança $U \subset \mathcal{N}\left(G_{1}\right)$ de $z$ tal que $\left\{g \in G_{1} / g U \cap U \neq \emptyset\right\}$ é finito. Como $\mathcal{N}\left(G_{1}\right)$ é precisamente invariante por $G_{1}$ em $\left\langle G_{1}, G_{2}\right\rangle$, então $g U \cap U \neq \emptyset$ para finitos $g \in\left\langle G_{1}, G_{2}\right\rangle$, com o qual $\left\langle G_{1}, G_{2}\right\rangle$ age descontinuamente no ponto $z \in \mathbb{H}$, $\operatorname{logo}$ em cada ponto de $\mathbb{H}$. Assim $\left\langle G_{1}, G_{2}\right\rangle$ é Fuchsiano.

Resta provar a afirmação $i$ iii). Observe-se que $I \subsetneq I_{1}$ e por hipotese, para $g \in G_{2}, \operatorname{Sta}_{G_{2}}(g I)=$ $g \cdot S t a_{G_{2}}(I) \cdot g^{-1} \neq S t a_{G_{2}}\left(I_{2}\right)=\left\langle g_{0}\right\rangle$, com o qual $g I \neq I_{2}$, para todo $g \in G_{2}$. Denote-se por $K$ ao subgrupo $S t a_{G_{2}}(I)$. Procuramos demostrar que $I$ é precisamente invariante por $K$ em $\left\langle G_{1}, G_{2}\right\rangle$, pois nesse caso, pelo Lema 1.2.15, $I$ sería um intervalo de descontinuidade de $\left\langle G_{1}, G_{2}\right\rangle$. 
Se $g \in G_{2} \backslash K, g I \cap I=\emptyset$ pela Proposição 1.2.14. Se $g \in G_{1} \backslash K$, suponha que $g \notin\left\langle g_{0}\right\rangle$ (pois nesse caso $\left.g \in G_{2} \backslash K\right)$, logo $g \notin S t a_{G_{1}}\left(I_{1}\right)$. Pela Proposição 1.2.14, $g I \subset g I_{1} \subset \hat{\mathbb{R}} \backslash I_{1} \subset \hat{\mathbb{R}} \backslash I$, logo $g I \cap I=\emptyset$. Em geral, se $g \in\left\langle G_{1}, G_{2}\right\rangle \backslash K$, pode-se supor que $g=a_{1} b_{1} a_{2} b_{2} \ldots a_{k} b_{k}, k \geq 1$, onde para cada $1 \leq i \leq k, a_{i} \in G_{1} \backslash\left\langle g_{0}\right\rangle$ e $b_{i} \in G_{2}$. Como $I$ e $I_{2}$ não são $G_{2}$-conjugados, $b_{k}$ transforma o intervalo $I$ num intervalo de descontinuidade de $G_{2}$ diferente de $I_{2}$. $a_{k}$ transforma $b_{k}(I)$ num intervalo contido num intervalo de descontinuidade $J_{0}$ de $G_{1}$ diferente de $I_{1}$ pois $a_{k} \notin\left\langle g_{0}\right\rangle$, com o qual $a_{k} b_{k}(I) \cap I=\emptyset$. Como $b_{k-1}\left(J_{0}\right) \subset b_{k-1}\left(I_{2}\right) \neq I$, tem-se que $b_{k-1} a_{k} b_{k}(I) \cap I=\emptyset$. Agora, se $b_{k-1}\left(I_{2}\right) \subset I_{1}$, como $a_{k-1} \notin\left\langle g_{0}\right\rangle=S t a_{G_{1}}\left(I_{1}\right)$, então $a_{k-1} b_{k-1}\left(I_{2}\right) \subset I_{2}$ e, se $b_{k-1}\left(I_{2}\right)=I_{2}$, então como $\Lambda_{G_{1}} \cap I_{1}=\emptyset$ e $\Lambda_{G_{1}}$ é $G_{1}$-invariante, $a_{k-1} b_{k-1} a_{k} b_{k}\left(I_{2}\right) \subset I_{2}$; em qualquer dos dois casos, $a_{k-1} b_{k-1} a_{k} b_{k}(I) \cap I=\emptyset$. Repetendo o argumento uma e outra vez conclui-se que $g I \cap I=\emptyset$ e com isto termina a prova da parte $i i i)$.

Corolario 1.2.19. Se $G_{1}, G_{2}<\mathrm{PSL}_{2}(\mathbb{R})$ são Fuchsianos, $g_{0}$ um elemento hiperbólico de bordo para $G_{1}$ e $G_{2}$ tal que $\mathcal{N}\left(G_{1}\right) \cap \mathcal{N}\left(G_{2}\right)=\mathcal{E}\left(g_{0}\right)$ e $h \in G_{2}$ é um elemento hiperbólico primitivo de bordo de $G_{2}$ tal que $\langle h\rangle$ e $\left\langle g_{0}\right\rangle$ não são $G_{2}$-conjugados, então $h$ é um elemento hiperbólico de bordo de $\left\langle G_{1}, G_{2}\right\rangle$.

Lembre-se que uma superfície de Riemann é uma variedade complexa de dimensão complexa igual à um. Se $f: S_{1} \longrightarrow S_{2}$ é uma aplicação entre superfícies de Riemann, dize-se que $f$ é um biholomorfismo se $f$ é holomorfa e bijetóra (nesse caso a inversa de $f$ também é holomorfa pelo Teorema da função inversa). Duas superfícies de Riemann são conformemente equivalentes se existe um biholomorfismo entre as superfícies. Pelo Teorema de uniformização, toda superfície de Riemann simplemente conexa é conformemente equivalente à $\hat{\mathbb{C}}$ ou à $\mathbb{C}$ ou ao plano hiperbólico $\mathbb{H}$, com o qual o recobridor universal de qualquer superfície de Riemann pode-se assumir que uma das três superfícies anteriores. A esfera de Riemann recobre somente a ela mesma, logo qualquer 2-esfera topológica com alguma estrutura complexa será conformemente equivalente à $\widehat{\mathbb{C}}$. O plano $\mathbb{C}$ recobre somente à $\mathbb{C}$, ao plano furado e aos toros. Todas as demais superfícies são recobertas pelo plano hiperbólico $\mathbb{H}$; nesse caso dize-se que a superfície é hiperbólica. Assim, se $S$ é uma superfície hiperbólica e se $\pi: \mathbb{H} \longrightarrow S$ denota a aplicação recobridora de $S$, é bem conhecido que a superfície $S$ é conformemente equivalente ao quociente $\mathbb{H} / G$, sendo $G$ o grupo de biholomorfismo $g: \mathbb{H} \longrightarrow \mathbb{H}$ tais que $\pi \circ g=g$; além disso $G$ é isomorfo ao grupo fundamental $\pi_{1}(S)$ da superfície e age descontinuamente no espaço $\mathbb{H}, \operatorname{logo} G$ é um grupo Fuchsiano que não contém elementos elíticos pois $\pi_{1}(S)$ é livre de torsão.

Definição 1.2.20. Seja $S$ uma superfície hiperbólica. Um grupo $G<\mathrm{PSL}_{2}(\mathbb{R})$ chama-se de um modelo Fuchsiano de $S$, se $G$ é um grupo Fuchsiano e $S$ é conformemente equivalente ao quociente $\mathbb{H} / G$. Denote-se por $\pi_{G}: \mathbb{H} \longrightarrow S$ à correspondente aplicação quociente. Se $G$ não é elementar, o núcleo de Nielsen de $S$ define-se como $\pi_{G}(\mathcal{N}(G))=\mathcal{N}(G) / G$.

Observação 1.2.21. i) O modelo Fuchsiano de uma superfície hiperbólica não é única, porém, se $G$ e $G^{\prime}$ são grupos Fuchsianos, eles são conjugados se, e somente se, as superfícies $\mathbb{H} / G$ e $\mathbb{H} / G^{\prime}$ são conformementes equivalentes.

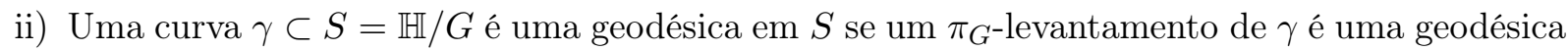
em $\mathbb{H}$; neste caso qualquer componente de $\pi_{G}^{-1}(\gamma)$ será uma linha geodésica de $\mathbb{H}$. Se $g \in G$ é um elemento hiperbólico primitivo, o eixo $\mathcal{E}_{g}$ é precisamente invariante pelo grupo $\langle g\rangle$, logo $\pi_{G}\left(\mathcal{E}_{g}\right)$ coincide com o quociente $\mathcal{E}_{g} /\langle g\rangle$ o qual é uma geodésica em $S$ fechada e simples. Recíprocamente, se $\gamma$ é uma geodésica fechada e simples em $S$, cada componente de $\pi_{G}^{-1}(\gamma)$ será o eixo de de um elemento hiperbólico primitivo de $G$ e, se $\mathcal{E}_{g_{1}}$ e $\mathcal{E}_{g_{2}}$ são duas componentes de $\pi_{G}^{-1}(\gamma)$, então os grupos $\left\langle g_{1}\right\rangle$ e $\left\langle g_{2}\right\rangle$ são $G$-conjugados. 
iii) Se $g \in G$ é um elemento parabólico, conjugando adequadamente $G$ pode-se assumir que $g(z)=z \pm 1$. O aberto $U=\{z \in \mathbb{H} / \operatorname{Im} z>c\}$ é um horodisco precisamente invariante pelo grupo $\langle g\rangle$ para $c$ suficientemente grande, $\operatorname{logo} \pi_{G}(U)=U /\langle g\rangle$, o qual é conformemente equivalente a um disco furado $\mathbb{D}^{*}=\{0<|z|<1\}$. Isto implica que se $G$ tem elementos parabólicos, $S$ tem furos.

iv) Dize-se que a superfície $S$ é do tipo finito se seu grupo fundamental é finitamente gerado; caso contrario a superfície é do tipo infinito. Observe-se que tanto $\hat{\mathbb{C}}$ como $\mathbb{C}, \mathbb{C}^{*}$ e qualquer toro são todos do tipo finito, logo uma superfície do tipo infinito só pode ser uma superfície hiperbólica e em consequência deve ser realizado por algum modelo Fuchsiano. Uma superfície de Riemann $S$ do tipo finito dize-se que é do tipo conforme $(g, n, b)$, se é conformemente equivalente à uma superfície fechada de gênero $g$ menos $n$ pontos e menos $b$ discos fechados (dize-se coloquialmente que $S$ têm $n$ "furos"e $b$ "buracos"). Observe-se que uma superfície do tipo $(g, n, b)$ é hiperbólica see $2 g+n+b>2$.

v) Se $S=\mathbb{H} / G$ seu núcleo de Nielsen $K=\mathcal{N}(G) / G$ resulta ser o menor subespaço fechado e convexo de $S$ tal que a inclusão $K \hookrightarrow S$ é uma equivalência homotópica. De fato, $K$ é uma subsuperfície de $S$ possívelmente com bordo: Se $G$ é do primeiro tipo, então $K=S$; quando $G$ é do segundo tipo, o eixo de cada elemento hiperbólico primitivo de bordo de $G$ projeta-se sobre uma componente de bordo da subsuperfície $K$. Em qualquer caso, $K$ contém todas as curvas geodésicas fechadas de $S$. A figura 1.5 mostra um desenho de uma superfície cujo núcleo de Nielsen tem dois componentes de bordo.

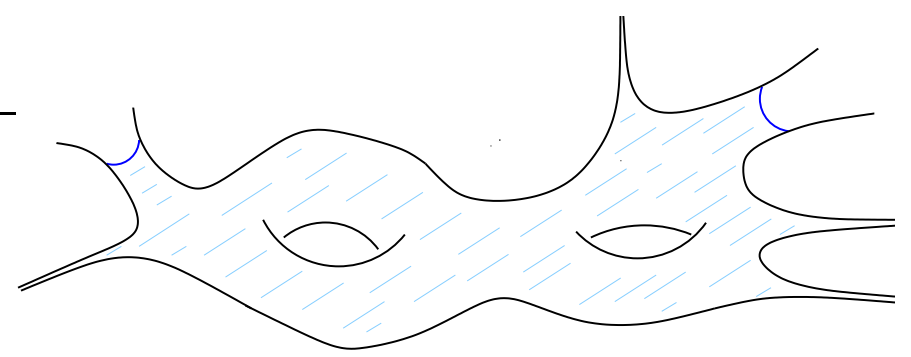

Figura 1.5: Núcleo de nielsen de uma superfície. Este têm duas geodésicas como componentes de bordo

Definição 1.2.22. A métrica de Poincaré ou métrica hiperbólica de uma superfície $S=\mathbb{H} / G$, a qual será denotada por $\lambda_{S}(z)|d z|$, define-se em cada ponto $z \in S$ por,

$$
\lambda_{S}(z):=\frac{\lambda_{\mathbb{H}}(w)}{\left|\pi_{G}^{\prime}(w)\right|}
$$

onde $w \in \mathbb{H}$ é um ponto qualquer tal que $\pi_{G}(w)=z$.

É fácil ver que na definição acima o valor $\lambda_{S}(z)$ independe da escolha do ponto $w \in \pi_{G}^{-1}(z)$. Uma vez conhecida a métrica de Poincaré de uma superfície $S$ é possível calcular o comprimento hiperbólico de uma curva $\gamma:[a, b] \longrightarrow S$; denotaremos por $\ell_{S}(\gamma)$ ao comprimento hiperbólico da curva $\gamma$. De maneira similar a como foi definido a distância $d_{\mathbb{H}}$, a métrica de Poincaré de $S$ determina uma distância $d_{S}$ na superfície $S$ fazendo que a projeção $\pi_{G}:\left(\mathbb{H}, d_{\mathbb{H}}\right) \longrightarrow\left(S, d_{S}\right)$ seja uma isometría local. A seguinte proposição, aínda que seja um resultado simple, revela-se de grande importância no desenvolvimento desta tese (ver o Lema 3.2.2)

Proposição 1.2.23. Considere-se uma superfície hiperbólica com sua métrica de Poincaré $\lambda_{S}(z)|d z|$, e suponha que $S_{0} \subset S$ é uma subsuperfície de $S$ com métrica de Poincaré $\lambda_{S_{0}}(z)|d z|$. Então $\lambda_{S_{0}}(z) \geq \lambda(z)$, para todo $z \in S_{0}$. Em particular, se $\gamma:[a, b] \longrightarrow S_{0}$ é uma curva parametrizada, então $\ell_{S_{0}}(\gamma) \geq \ell_{S}(\gamma)$. 
O seguinte resultado devido a A. Basmajian (Ver [Bas93]), Teorema 3, permite colar uma sequência de superfícies hiperbólicas até obter uma nova superfície mais complexa. Referimo-nos ao artigo acima mencionado para ver a demostração deste teorema.

Teorema 1.2.24. Seja $\left\{G_{n}\right\}_{n \geq 0}$ uma familia de grupos Fuchsianos que satisfazem as seguintes propriedades para cada $n \geq 0$ :

1) $g_{n}$ e $g_{n+1}$ são elementos hiperbólicos primitivos de bordo do grupo $G_{n}$.

2) Os grupos cíclicos $\left\langle g_{n}\right\rangle$ e $\left\langle g_{n+1}\right\rangle$ não são $G_{n}$-conjugados.

3) $\mathcal{N}\left(G_{n}\right) \cap \mathcal{N}\left(G_{n+1}\right)=\mathcal{E}_{g_{n+1}}$.

Se $G:=\left\langle G_{n}\right\rangle_{n=0}^{+\infty}$, então,

a) Gé um grupo Fuchsiano.

b) $\left\{\pi_{G}\left(\mathcal{E}_{g_{n}}\right)\right\}$ é uma família de geodésicas fechadas, simples, duas a duas disjuntas, cada uma das quais separa a superficie $\mathbb{H} / G$.

c) $\mathcal{N}(G) / G$ é a união topológica dos núcleos de Nielsen $\left\{\mathcal{N}\left(G_{n}\right) / G_{n}\right\}_{n \geq 0}$, os quais são colados consecutivamente no longo das curvas $\pi_{G}\left(\mathcal{E}_{g_{n}}\right)$.

d) Cada superfície $\mathcal{N}\left(G_{n}\right) / G_{n}$ está isométricamente mergulhada na superfície $\mathcal{N}(G) / G$.

Da mesma forma como Basmajian usa este teorema para definir suas superfícies flauta, usaremos este resultado na seção 1.4 para definir as superfícies que chamaremos de gaita de fole. Antes de passar para a próxima seção, consideraremos um tipo especial de superfície hiperbólica chamadas de calças (alguns autores chaman-lhe de $Y$-peças ou de trousser)

Definição 1.2.25. Uma calça é uma superfície de Riemann do tipo conforme $(0, n, b)$, onde $n+b=3$ e $b \geq 1$. Se $b \neq 3$, dize-se que a calça é justa.

Observação 1.2.26. $\quad$ i) Topológicamente uma calça é o dominio do plano

$$
\{|z|<2\} \backslash\left(\left\{|z-1|<\frac{1}{2}\right\} \cup\left\{|z+1|<\frac{1}{2}\right\}\right)
$$

Na literatura usual uma calça é definido como uma superfície com bordo; isto corresponde ao núcleo de Nielsen de uma calça como está definida acima. Nesta tese não faremos diferença entre a calça e seu núcleo a menos que isto seja necessario.

ii) Dado qualquer terna de números reais positívos $\left(\ell_{1}, \ell_{2}, \ell_{3}\right)$, existe uma única calça, a menos de isometría, cujas componentes de bordo (do seu núcleo de Nielsen) são geodésicas de comprimento $\ell_{1}, \ell_{2}$ e $\ell_{3}$ respectivamente. Este mesmo resultado é aínda válido quando a calça é justa; neste caso considera-se que um furo tem comprimento $\ell_{i}=0$. A figura 1.6 mostra o desenho de três calças, sendo as dois da direita calças justas.

iii) Numa calça $S$ pode-se escolher o modelo Fuchsiano de $S$ como o grupo $G$ gerado pelos elementos $g_{0}, g_{1} \in \mathrm{PSL}_{2}(\mathbb{R})$ de maneira que: 

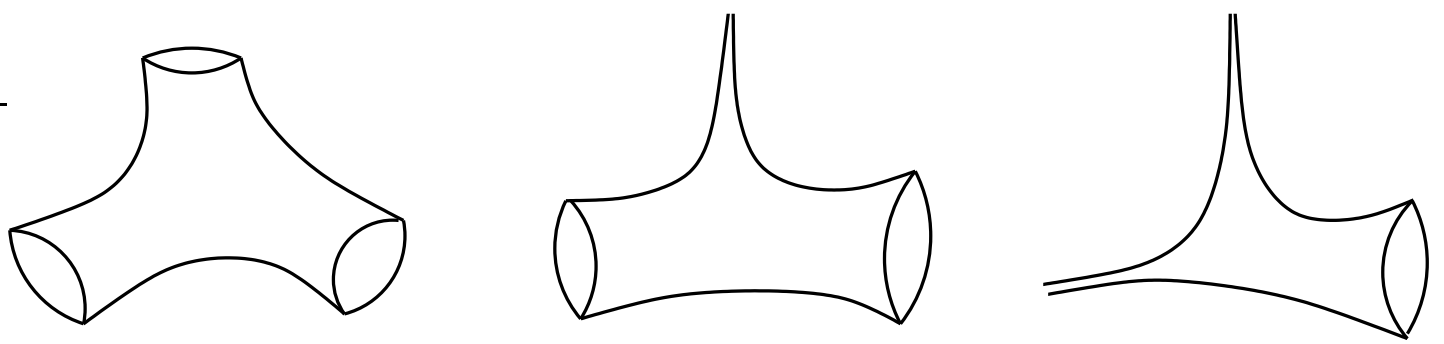

Figura 1.6

1. Se $S$ é do tipo $(0,0,3), g_{0}$ e $g_{1}$ são elementos hiperbólicos primitivos de bordo que representam duas das componentes de bordo da calça e $g_{0} g_{1}$ é um elemento hiperbólico que representa a terceira componente de bordo da calça.

2. Se $S$ é do tipo $(0,1,2), g_{0}$ e $g_{1}$ são elementos hiperbólicos como acima, mas $g_{0} g_{1}$ é um elemento parabólico que representa o furo da calça.

3. Se $S$ é do tipo $(0,2,1), g_{0}$ é um elemento parabólico representando um furo de $S, g_{1}$ é um elemento hiperbólico primitivo de bordo que representa uma das componentes de bordo de $S$ e $g_{0} g_{1}$ é um elemento parabólico que representa o segundo furo de $S$.

Em qualquer dos três casos acima dize-se que $\left(g_{0}, g_{1}\right)$ é um par de geradores estándar da calça.

As calças são importantes pois elas são consideradas as peças fundamentais na construção de superfícies hiperbólicas no seguinte sentido: Se $S$ é uma superfície hiperbólica do tipo finito, é possível escolher uma família finita $\Gamma$ de curvas geodésicas em $S$ fechadas e simples tal que $S \backslash \Gamma$ é uma união disjunta de calças; na figura 1.7 mostra-se um exemplo de decomposição em calças de uma superfície do tipo $(2,4,2)$.

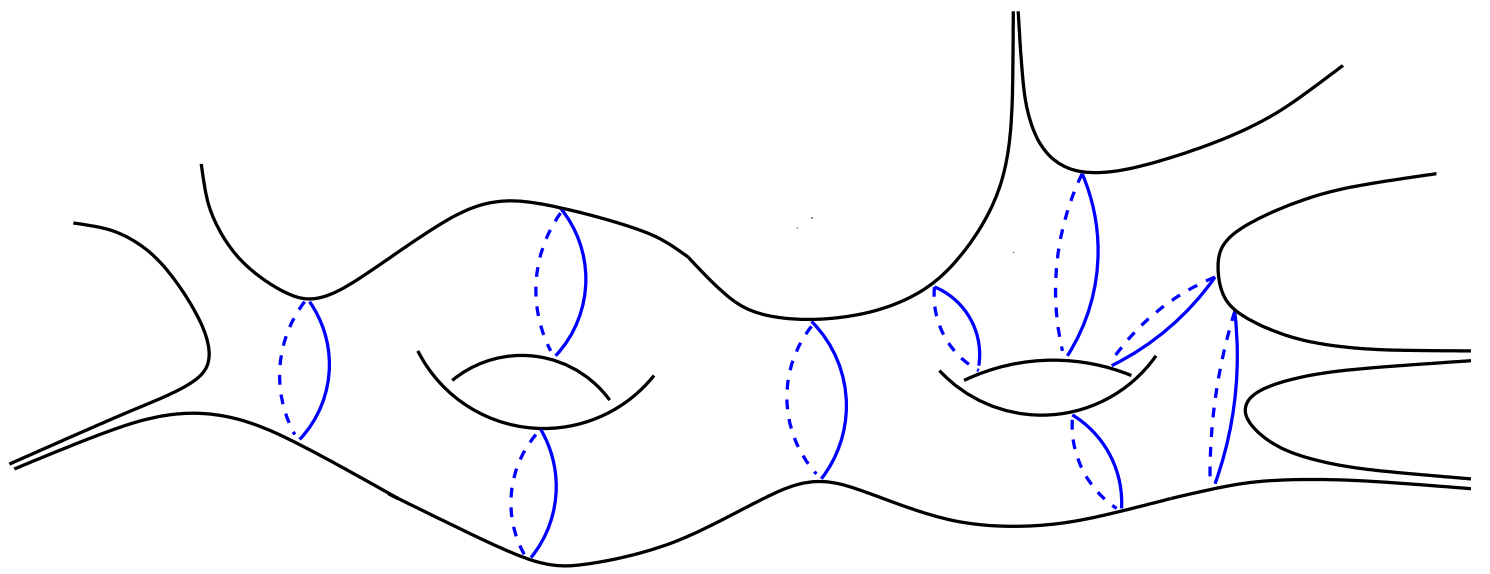

Figura 1.7: Decomposição de uma superfície em 8 calças, 3 delas sendo calças justas

Porém, numa superfície do tipo infinito pode acontecer que não exista uma multicurva $\Gamma$ que decompõe a superfície somente em calças (ver por exemplo a superfície na figura 3.6.3 do [J.H06]). O que sim é possível é decompor qualquer superfície em peças básicas que são, ou calças ou peças simplemente conexas.

Proposição 1.2.27 (Ver [J.H06]). Seja S uma superfície hiperbólica diferente de $\mathbb{H}$, de um cilindro ou de um disco furado. Existe em $S$ uma família $\Gamma$ de geodésicas fechadas simples as quais não são homotópicas entre sí, tal que, se $\bar{\Gamma}$ denota o fecho da união dessas curvas, então cada componente de $S \backslash \bar{\Gamma}$ é conformemente equivalente ou a uma calça ou a um semiplano. Aliás, as peças equivalentes a semiplanos são limitados por componentes de $\bar{\Gamma} \backslash \Gamma$. 
Abusando um pouco do idioma chamaremos de decomposição em calças de uma superfície a uma multicurva como na Proposição 1.2.27, ainda alguns das componentes do complementar da multicurva seja um semiplano. É importante mencionar que as peças na decomposição em calças serão calças mesmas, por exemplo quando o modelo Fuchsiano da superfície é do primeiro tipo.

\subsection{Curvas geodésicas em superfícies hiperbólicas}

Considere-se uma superfície hiperbólica $S$. Uma curva fechada $\gamma \subset S$ é primitivo se a classe de homotopía $c=[\gamma]$ não é potencia de um elemento diferente de $\pi_{1}(S)$. Observe-se que se $S=\mathbb{H} / G$ e $g \in G$ representa a classe de homotopía de $\gamma$, então $\gamma$ é primitivo se, e somente se, $g$ é primitivo como um elemento em $G$. Dize-se que uma curva fechada $\gamma$ é essencial, se $\gamma$ não é homotópica a um ponto nem homotópica a um furo de $S$. Se um elemento hiperbólico $z \mapsto \frac{a z+b}{c z+d}$, $a d-b c=1$, representa uma clase de homotopía de uma geodésica fechada $\gamma \subset S$, o traço de $\gamma$ é $\operatorname{Tra}(\gamma):=a+d$. Suponha que $a$ e $b$ são duas classes de homotopía de curvas fechadas simples em $S$. O número de interseção (geométrica) entre a e b, que será denotado por $i(a, b)$, é o mínimo número de interseções entre um representante na classe $a$ e um representante na classe $b$. Observe-se que $i(a, b)=i(b, a)$ e que $i(a, a)=0$. Por outro lado se um representante de $a$ separa a superfície em dois componentes conexas, então para qualquer classe $b$, tem-se que $i(a, b)$ é um número par. A seguinte observação lista uma serie de propriedades bem conhecidas

Proposição 1.3.1 (Ver [Bea83, Bus92, FM11, J.H06]). ㄴ) Se $\gamma \subset S$ é uma curva fechada simples não homotópica a um ponto, então $\gamma$ é primitivo.

ii) Se $\gamma \subset S$ é uma curva geodésica fechada homotópica a uma curva fechada simples, então $\gamma$ também é simples.

iii) Na classe de homotopía de uma curva fechada essencial em $S$ existe uma única curva geodésica fechada $\gamma$. Além disso $\ell_{S}(\gamma)$ realiza o mínimo dos comprimentos hiperbólicos das curvas na classe $[\gamma]$.

iv) Se $\gamma$ é uma geodésica fechada em $S$, então $\operatorname{Tra}^{2}(\gamma)=4 \cosh ^{2}\left(\frac{\ell_{S}(\gamma)}{2}\right)$

v) Sejam a e b classes de homotopía diferentes de duas curvas fechadas simples em $S$ e suponha que $\alpha$ e $\beta$ são as geodésicas em a e b respectivamente. Então $i(a, b)$ coincide com o número de interseções entre as curvas $\alpha$ e $\beta$.

Observe-se que em particular a Proposição 1.3 .1 v) implica o seguinte: Se $\alpha_{1}, \beta_{1}$ são duas curvas fechadas e simples que não são homotópicas, e se $\alpha$ e $\beta$ são as geodésicas nas classes de homotopía de $\alpha_{1}$ e $\beta_{1}$ respectivamente então, $\alpha_{1} \cap \beta_{1}=\emptyset$ implica que $\alpha \cap \beta=\emptyset$.

A continuação serão mostrados alguns resultados relativos a certas superfícies hiperbólicas simples que fazem um papel importante no desenvolvimento do capítulo 3

Definição 1.3.2. Um dominio anular ou simplemente um anel é uma superfície de Riemann cujo grupo fundamental é cíclico infinito.

Se $A$ é um anel qualquer, então acontece somente uma das seguintes possibilidades, ver [J.H06], Proposição 3.2.1.

i) $A$ é conformemente equivalente à $\mathbb{C}^{*}$. 
i) $A$ é conformemente equivalente à $\mathbb{D}^{*}$.

i) $A$ é conformemente equivalente à $A_{M}$, para algum $M>0$, onde

$$
A_{M}:=\left\{z \in \mathbb{C} / 1<|z|<e^{2 \pi M}\right\}
$$

Nos primeiros dois casos dize-se que o anel tem módulo infinito, enquanto que no caso iii), o módulo do anel $A$, denotado por Mod $A$, define-se como o número real positivo $M$. É fácil ver que dois aneis de módulo finito são biholomorfos se, e somente se, têm o mesmo módulo.

Proposição 1.3.3. Num anel $A$ de módulo finito existe uma única geodésica fechada que denotaremos por $\gamma_{A}$, a qual é simples e separa as duas componentes de fronteira de A. Além disso, o comprimento hiperbólico de $\gamma_{A}$ é:

$$
\ell_{A}\left(\gamma_{A}\right)=\frac{\pi}{\operatorname{Mod} A}
$$

Determinar se um anel tem módulo finito é uma tarefa fácil, porém conhecer qual é seu módulo, salvo em casos excepcionais, é difícil pois em principio precisa-se conseguir o biholomorfismo entre o anel e o respectivo anel $A_{M}$. A teoría dos comprimentos extremais permite estimar o módulo de um anel arbitrario e, em conseqüência, estimar o comprimento de sua curva geodésica. Suponha que $A$ é um anel. Uma densidade Boreliana em $A$ é uma função Borel-mensurável $\rho: A \longrightarrow \mathbb{R}$ tal que $\rho \geq 0$ e a $\rho$-área de $A$, definida por

$$
\mathcal{A}_{\rho}(A):=\iint_{A} \rho(z)^{2} d x d y
$$

seja positivo. Denote-se por $m_{A}$ a coleção de todas as densidades Borelianas de $A$. Se $\Gamma_{A}$ é a família de todas as curvas no anel $A$ que conectam as duas componentes de fronteira do $A$, a $\rho$-distância entre essas componentes para algum $\rho \in m_{A}$ define-se como,

$$
\mathcal{L}_{\rho}(A):=\inf _{\gamma \in \Gamma_{A}} \int_{\gamma} \rho(z)|d z|
$$

O seguinte teorema (ver [Ahl73], capítulo 4) determina o módulo de um anel usando as densidades Borelianas dele.

Teorema 1.3.4. Seja A um anel, então

$$
\operatorname{Mod} A=\sup _{\rho \in m_{A}} \frac{\mathcal{L}_{\rho}(A)^{2}}{\mathcal{A}_{\rho}(A)}
$$

Seja $\gamma$ uma geodésica fechada simples numa superfície hiperbólica $S$. Dado $r>0$, a vizinhança $\mathbb{A}(\gamma, r):=\left\{x \in S / d_{S}(x, \gamma)<r\right\}$ chama-se de colar de $\gamma$ se $\mathbb{A}(\gamma, r)$ é um anel mergulhado em $S$; nesse caso o raio $r$ é a largura do colar. Considere-se a função decrescente $\eta:(0,+\infty) \longrightarrow(0,+\infty)$ onde, para cada $\ell>0$,

$$
\eta(\ell):=\log \left(1+\frac{2}{e^{\ell / 2}-1}\right)
$$

A função $\eta$ será chamada simplemente de função colar. O seguinte resultado, chamado o lema do colar, é devido a L. Keen, ver [Kee74]. 
Teorema 1.3.5. Seja $\gamma$ uma geodésica fechada simples em $S$, então o subconjunto $\mathbb{A}_{\gamma}:=\mathbb{A}\left(\gamma, \eta\left(\ell_{S}(\gamma)\right)\right)$ é um colar. Além disso, se $\gamma_{1}$ e $\gamma_{2}$ são duas curvas geodésicas fechadas simples diferentes, os respectivos colares $\mathbb{A}_{\gamma_{1}}$ e $\mathbb{A}_{\gamma_{2}}$ de largura $\eta\left(\ell_{S}\left(\gamma_{1}\right)\right)$ e $\eta\left(\ell_{S}\left(\gamma_{2}\right)\right)$ respectivamente, são disjuntos.

Observação 1.3.6. Em diante $\mathbb{A}_{\gamma}$ denotará o colar da curva $\gamma$ de largura $\eta\left(\ell_{S}(\gamma)\right)$. Claramente $\mathbb{A}(\gamma, r)$ é um colar para todo $0<r \leq \eta\left(\ell_{S}(\gamma)\right)$ e, é possível que aínda seja para algum $r>\eta\left(\ell_{S}(\gamma)\right)$, sem embargo a função colar é maximal em geral no sentido que dá o máximo valor que garante as conclusões do teorema acima. Por exemplo, numa calça justa do tipo $(0,1,2)$, se $\gamma_{1}$ e $\gamma_{2}$ são as geodésicas do bordo de seu núcleo de Nielsen, então a distância hiperbólica entre $\gamma_{1}$ e $\gamma_{2}$ é exatamente $\eta\left(\ell_{S}\left(\gamma_{1}\right)\right)+\eta\left(\ell_{S}\left(\gamma_{2}\right)\right)$.

Corolario 1.3.7. Sejam $\gamma$ e $\beta$ duas geodésicas fechadas, simples e diferentes numa superfície hiperbólica $S$. Se $\beta \cap \mathbb{A}_{\gamma} \neq \emptyset$, então $\beta \cap \gamma \neq \emptyset$.

Considere-se agora uma superfície hiperbólica $S$ conformemente equivalente a um dominio de $\hat{\mathbb{C}}$ diferente da esfera menos três pontos, com o qual não há perda de generalidade em supor que $S=\hat{\mathbb{C}} \backslash X$, onde $X$ é um fechado com pelo menos quatro pontos. Suponha que $X$ é a união disjunta de dois subconjuntos fechados $D$ e $E$, cada um dos quais têm pelo menos dois pontos. Em geral podem existir diferentes geodésicas fechadas e simples em $S$ que separam os conjuntos $D$ e $E$, é dizer, tais que o complemento em $\hat{\mathbb{C}}$ da curva tenha duas componentes, uma contendo à $D$ e a outra à $E$. Uma condição suficiente para garantir a unicidade da geodésica que satisfaz a propriedade anterior é a seguinte.

Proposição 1.3.8 (Ver [Com11], Teorema 1.5). Se $S=\hat{\mathbb{C}} \backslash(D \cup E)$, onde $D$ e E são fechados que contém pelo menos dois pontos cada um e onde $D$ é um subconjunto conexo, então existe uma única curva geodésica fechada simples $\gamma \subset S$ que separa $D$ de $E$.

Proposição 1.3.9 (Ver [Com11]). Seja $S=\hat{\mathbb{C}} \backslash X$, onde $X=D_{1} \cup E_{1}=D_{2} \cup E_{2}, D_{i}$, $E_{i}$ são fechados e com pelo menos dois pontos cada um, para $i=1,2$, e $D_{1}$ é conexo, propriamente contido em $D_{2}$. Sejam $\gamma_{1}$ e $\gamma_{2}$ curvas geodésicas fechadas simples que separam $D_{1}$ de $E_{1}$ e $D_{2}$ de $E_{2}$, respectivamente. Então $\gamma_{1}$ está contido na componente de $\hat{\mathbb{C}} \backslash \gamma_{2}$ que contém $D_{2}$. Em particular $\gamma_{1}$ e $\gamma_{2}$ são disjuntos.

Um caso especial é quando a superfície $S$ é do tipo conforme $(0, n, b)$ com $n+b=4$. Alguns autores chaman a tal superfície de $X$-peça (Naturalmente os mesmos autores que chaman de $Y$-peças as calças!). Observe-se que esta superfície é obtida ao colar duas calças como mostra a figura 1.8. No núcleo de Nielsen de $S$ existem cinco geodésicas simples quando $n=0$ : quatro delas são as componentes de bordo de comprimento $\ell_{i}, i=1,2,3,4$, e a outra, de comprimento hiperbólico $\ell$ é a geodésica que decompõe $S$ em duas calças.

A superfície $S$ fica determinada por os comprimentos $\ell, \ell_{i}, i=1,2,3,4$, e por um parámetro adicional $\tau$ chamado de torção o qual corresponde à torção feita ao colar ambas calças ao longo da geodésica central de comprimento $\ell$; os seis parámetros anteriores correspondem com as coordenadas de Fenchel-Nielsen da superfície $S$, ver [J.H06] ou [YT92] para mais detalhes sobre estas coordenadas 

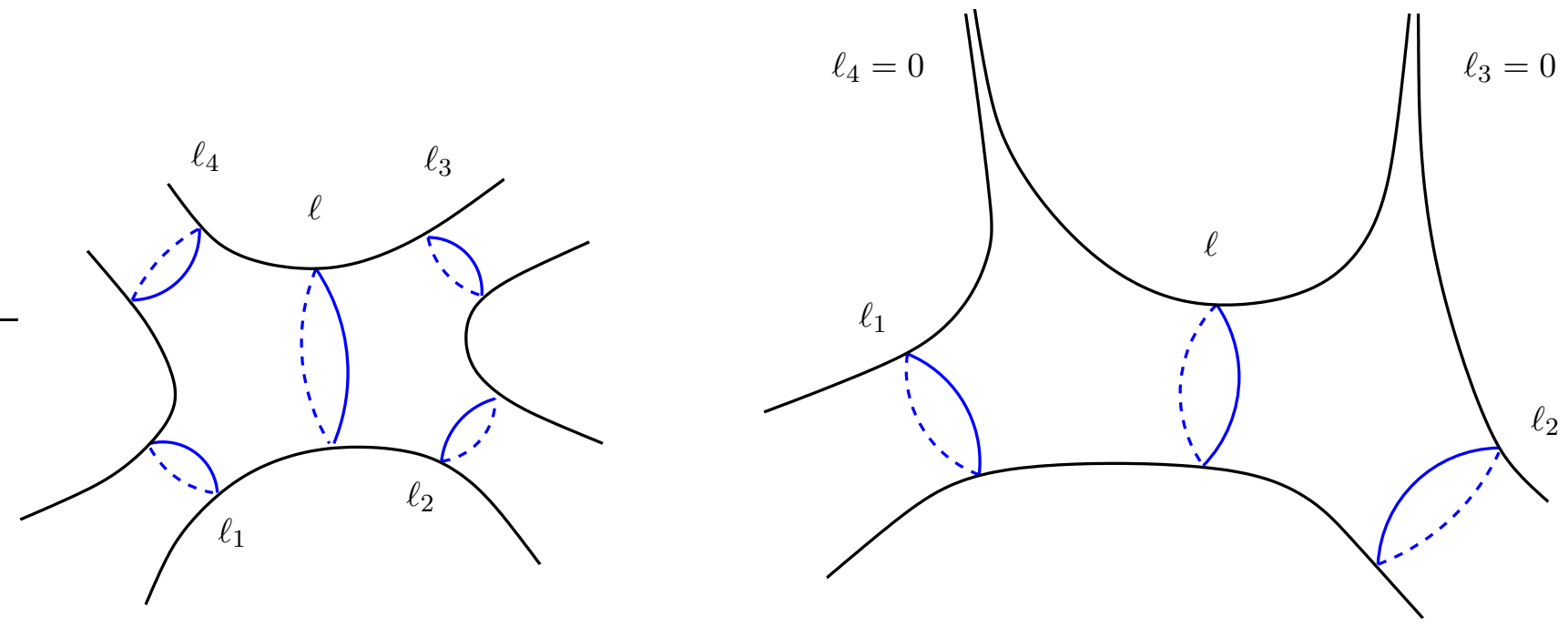

Figura 1.8: Desenho de dois superfícies, uma do tipo $(0,0,4)$ e a outra do tipo $(0,2,2)$

em qualquer superfície hiperbólica. O mesmo vale quando $n \geq 1$ mas nesse caso o número de coordenadas de comprimento é menor; aínda assim toma-se o valor $\ell_{i}=0$ quando este corresponda a um furo. Uma maneira simple de definir a coordenada de torção é a seguinte: Se $\gamma, \gamma_{1}$ e $\gamma_{2}$ são as geodésicas de comprimento $\ell, \ell_{1}$ e $\ell_{2}$ respectivamente, podem-se levantar para $\mathbb{H}$ a três linhas geodésicas $L, L_{1}$ e $L_{2}$ as quais estão aninhadas como mostra a figura 1.9.

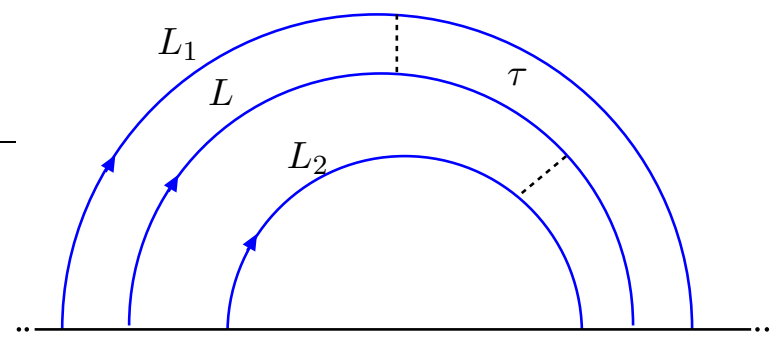

Figura 1.9

Considere-se $z^{-}$o ponto de interseção entre a geodésica ortogonal à $L$ e $L_{1}$ com a linha $L$, e $z^{+}$ o ponto de interseção entre a geodésica ortogonal à $L$ e $L_{2}$ com a linha $L$. Então $\tau:= \pm d_{\mathbb{H}}\left(z^{-}, z^{+}\right)$, onde o sinal será positivo se o segmento geodésico $\left[z^{-}, z^{+}\right]$tem a mesma orientação da geodésica $L$, e será negativo no caso contrario. Suponha agora que $\beta$ é uma geodésica fechada e simples como mostra a figura 1.10. Em [Oka93] é escrito uma fórmula que expresa o comprimento hiperbólico da curva $\beta$ em função das coordenadas de Fenchel-Nielsen anteriormente descritas para o caso $n=0$; em [APLS11] prova-se que a mesma fórmula vale no caso $n>0$ tomando os respectivos parámetros de comprimento igual à 0 .

Proposição 1.3.10 (Ver [Oka93], [APLS11]). Nas superfícies $S$ mostrada na figura 1.10 , se $\ell_{i}=$ $\ell_{S}\left(\gamma_{i}\right), i=1,2,3,4, \ell=\ell_{S}(\gamma), \ell^{*}=\ell_{S}(\beta)$ e $\tau$ é a coordenada de torção relativo a curva $\gamma$, então, 


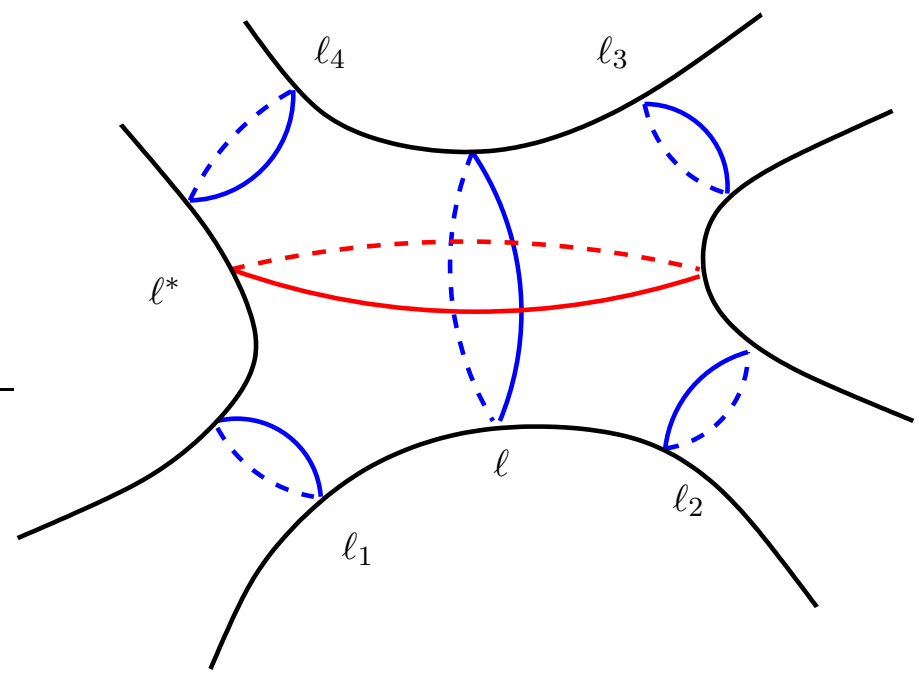

Figura 1.10

$$
\begin{aligned}
\cosh \left(\frac{\ell^{*}}{2}\right) \cdot \sinh ^{2}\left(\frac{\ell}{2}\right)= & \cosh \left(\frac{\ell_{1}}{2}\right) \cdot \cosh \left(\frac{\ell_{2}}{2}\right)+\cosh \left(\frac{\ell_{3}}{2}\right) \cdot \cosh \left(\frac{\ell_{4}}{2}\right) \\
& +\cosh \left(\frac{\ell}{2}\right) \cdot\left[\cosh \left(\frac{\ell_{1}}{2}\right) \cdot \cosh \left(\frac{\ell_{3}}{2}\right)+\cosh \left(\frac{\ell_{2}}{2}\right) \cdot \cosh \left(\frac{\ell_{4}}{2}\right)\right] \\
& +\cosh (\tau) \cdot\left[\cosh ^{2}\left(\frac{\ell}{2}\right)+2 \cosh \left(\frac{\ell_{1}}{2}\right) \cdot \cosh \left(\frac{\ell_{4}}{2}\right) \cdot \cosh \left(\frac{\ell}{2}\right)\right. \\
& \left.+\cosh ^{2}\left(\frac{\ell_{1}}{2}\right)+\cosh ^{2}\left(\frac{\ell_{4}}{2}\right)-1\right]^{1 / 2} \times\left[\cosh ^{2}\left(\frac{\ell}{2}\right)\right. \\
& \left.+2 \cosh \left(\frac{\ell_{2}}{2}\right) \cdot \cosh \left(\frac{\ell_{3}}{2}\right) \cdot \cosh \left(\frac{\ell}{2}\right)+\cosh ^{2}\left(\frac{\ell_{2}}{2}\right)+\cosh ^{2}\left(\frac{\ell_{3}}{2}\right)-1\right]^{1 / 2}
\end{aligned}
$$

Quando $S$ é do tipo $(0,2,2)$ como na figura 1.8 à direita, tomando $\ell_{3}=\ell_{4}=0$ a fórmula acima simplifica-se considerablemente. Neste caso tem-se:

Corolario 1.3.11. Nas condiçôes acima, onde $S$ é do tipo conforme $(0,2,2)$, tem-se,

$$
\begin{aligned}
\cosh \left(\frac{\ell^{*}}{2}\right) \cdot \sinh ^{2}\left(\frac{\ell}{2}\right)= & 1+\cosh \left(\frac{\ell_{1}}{2}\right) \cdot \cosh \left(\frac{\ell_{2}}{2}\right)+\cosh \left(\frac{\ell}{2}\right) \cdot\left[\cosh \left(\frac{\ell_{1}}{2}\right)+\cosh \left(\frac{\ell_{2}}{2}\right)\right] \\
& +\cosh (\tau) \cdot\left[\cosh \left(\frac{\ell}{2}\right)+\cosh \left(\frac{\ell_{1}}{2}\right)\right] \cdot\left[\cosh \left(\frac{\ell}{2}\right)+\cosh \left(\frac{\ell_{2}}{2}\right)\right]
\end{aligned}
$$

Em conseqüência,

$$
1+\cosh (\tau)=\frac{\sinh ^{2}\left(\frac{\ell}{2}\right) \cdot\left[\cosh \left(\frac{\ell^{*}}{2}\right)+1\right]}{\left[\cosh \left(\frac{\ell}{2}\right)+\cosh \left(\frac{\ell_{1}}{2}\right)\right] \cdot\left[\cosh \left(\frac{\ell}{2}\right)+\cosh \left(\frac{\ell_{2}}{2}\right)\right]}
$$

Para finalizar a seção, alguns comentários sobre o espaço de Teichmüller de uma superfície hiperbólica, aínda este não seja um tema central no desenvolvimento da tese. Fixando uma superfície 
de Riemann $S$, a qual assumimos que seja hiperbólica, considere-se a coleção dos pares $(R, f)$, onde $R$ é outra superfície de Riemann e $f: S \longrightarrow R$ é um homeomorfismo quaseconforme. O espaço de Teichmüller quaseconforme $\mathcal{T}(S)$ é o quociente desta coleção pela relação de equivalência onde dois pares $\left(R_{1}, f_{1}\right)$ e $\left(R_{2}, f_{2}\right)$ são equivalentes se $f_{2} \circ f_{1}^{-1}: R_{1} \longrightarrow R_{2}$ é homotópica a um biholomorfismo.

A distância de Teichmüller, denotada por $d_{\mathcal{T}}$, define-se da seguinte maneira: Se $\left[R_{1}, f_{1}\right]$ e $\left[R_{2}, f_{2}\right]$ são duas classes em $\mathcal{T}(S)$, então

$$
d_{\mathcal{T}}\left(\left[R_{1}, f_{1}\right],\left[R_{2}, f_{2}\right]\right):=\inf _{f} \log K(f),
$$

onde o ínfimo é tomado sobre todos os homeomorfismos quaseconformes $f: R_{1} \longrightarrow R_{2}$ homotópicos à $f_{2} \circ f_{1}^{-1}$ e $K(f)$ denota a dilatação máxima da aplicação $f$. Com a distância de Teichmüller o espaço $\left(\mathcal{T}(S), d_{\mathcal{T}}\right)$ resulta completo (Ver [ALPS12b]).

Outra distância em $\mathcal{T}(S)$ é a chamada distância de comprimento espectral: Denote-se por $\Sigma_{S}$ a coleção de todas as geodésicas fechadas na superfície $S$, então a distância de comprimento espectral que denotamos por $d_{\mathcal{L}}$ definese como,

$$
d_{\mathcal{L}}\left(\left[\left(R_{1}, f_{1}\right],\left[R_{2}, f_{2}\right]\right):=\log \max _{\gamma \in \Sigma_{S}}\left\{\frac{\ell_{S_{1}}\left[f_{1}(\gamma)\right]}{\ell_{S_{2}}\left[f_{2}(\gamma)\right]}, \frac{\ell_{S_{2}}\left[f_{2}(\gamma)\right]}{\ell_{S_{1}}\left[f_{1}(\gamma)\right]}\right\},\right.
$$

onde $\ell_{S_{i}}\left[f_{i}(\gamma)\right]$ denota o comprimento hiperbólico na superfície $S_{i}$ da geodésica na classe de homotopía da curva $\left[f_{i}(\gamma)\right]$.

Se $S$ é uma superfície do tipo finito as duas métricas são topológicamente equivalentes; este resultado foi conjeturado por T. Sorvali e foi provado em 1999 por L. Liu em [Lix99]; ele a sua vez conjeturó que o mesmo resultado é valido para superfícies do tipo infinito. Em [Shi03], H. Shiga constroi em cambio uma superfície de tipo infinito onde ambas métricas não são topológicamente equivalentes; além disso no mesmo artigo ele dá o seguinte teorema.

Teorema 1.3.12 ([Shi03]). Seja $S$ uma superfície hiperbólica tal que $S=\bigcup_{k \geq 1} P_{k}$, onde cada $P_{k}$ é uma calça (Algumas das quais podem ser justa). Se existe $M>0$ tal que, para toda curva $\gamma$ que seja uma componente de bordo de alguma das calças $P_{k}$, tem-se,

$$
\frac{1}{M}<\ell_{S}(\gamma)<M
$$

então as distâncias $d_{\mathcal{T}}$ e $d_{\mathcal{L}}$ geram a mesma topología em $\mathcal{T}(S)$.

Por outro lado uma condição necessaria para que estas métrica definam a mesma topología em $\mathcal{T}(S)$ é a seguinte.

Proposição 1.3.13 ([LSW08]). Se existe uma sequência de curvas geodésicas $\left(\gamma_{k}\right)_{k \geq 1}$ em $S$ tais que $\lim _{k \rightarrow+\infty} \ell_{S}\left(\gamma_{k}\right)=0$, então as métricas $d_{\mathcal{T}}$ e $d_{\mathcal{L}}$ não são topológicamente equivalentes.

Existem outros resultados referentes a teoría de Teichmüller em superfícies do tipo infinito, alguns deles generalizam os dois resultados acima (Ver por exemplo [Kin11]), alguns estudam outros espaços de Teichmüller como por exemplo o espaço de Teichmüller do comprimento espectral ou o espaço de Teichmüller com coordenadas de Fenchel-Nielsen, etc (Ver [ALP $\left.{ }^{+} 11\right]$, [ALPS12b], [ALPS16]). 


\subsection{Superfícies flauta e superfícies gaita de fole}

De maneira simple uma superfície flauta consiste em uma sequência infinita de calças conectadas uma seguida da outra como mostram as figuras 1.11 e 1.12 abaixo.

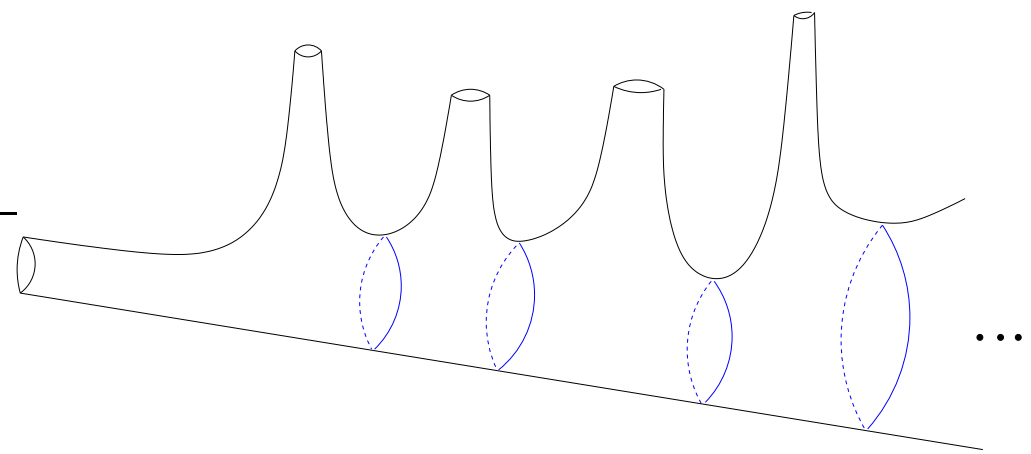

Figura 1.11: Superfície flauta

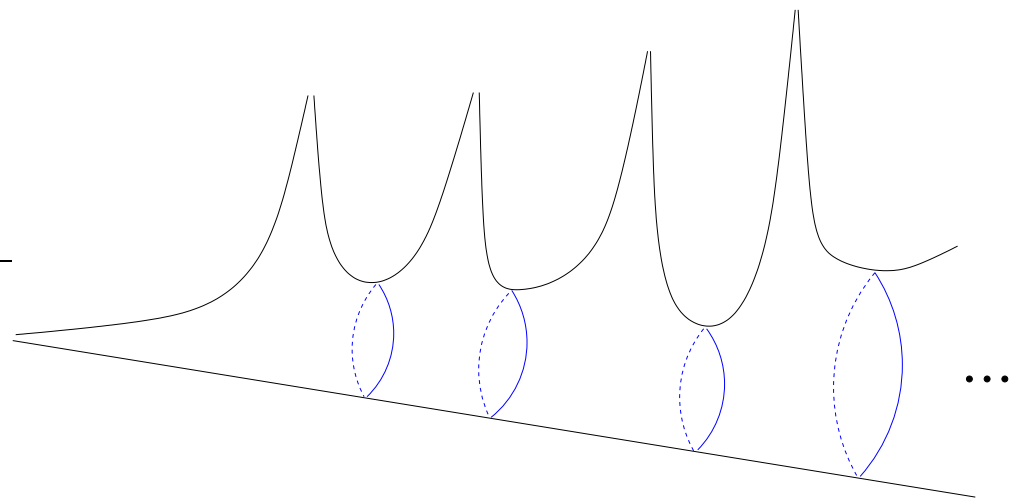

Figura 1.12: Superfície flauta justa

Estas superfícies aparecem estudiadas com detalhe pela primeira vez no artigo [Bas93] onde são consideradas como as superfícies do tipo infinito mais simples, embora com propriedades muito interesantes. Uma superfície gaita de fole é uma generalização do conceito de flauta cuja motivação reside no estudo das chamadas esferas de papel furadas que serão tratadas no capítulo 3. O desenho na figura 1.13 mostra um exemplo de gaita de fole. Este é obtido ao colar flautas numa esfera com um número finito de buracos, daí seu nome.

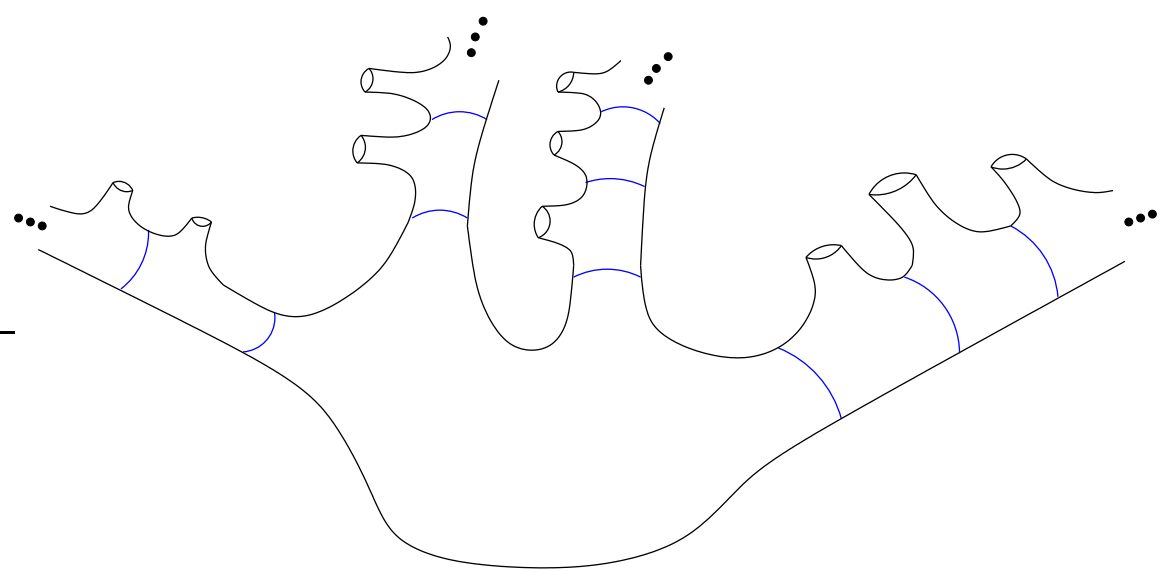

Figura 1.13: Gaita de fole de ordem 4. 
Esta seção comença com a definição de superfície flauta e algumas propriedades delas. Logo serão definidos as superfícies gaita de fole e serão generalizadas algumas das propriedades válidas para flautas.

Definição 1.4.1 ([Bas93]). Uma superfície flauta é uma superfície hiperbólica $S$ cujo modelo Fuchsiano $G$ é gerado por uma sequência de grupos Fuchsianos $\left\{G_{n}\right\}_{n \geq 0}$, sendo cada $G_{n}$ a sua vez o modelo Fuchsiano de uma calça com geradores estándar $\left(g_{n}, g_{n+1}\right)$ e tal que para cada $n \geq 1$ é satisfeita a seguinte propriedade:

$$
\mathcal{N}\left(G_{n}\right) \cap \mathcal{N}\left(G_{n+1}\right)=\mathcal{E}_{g_{n+1}}
$$

Dize-se que a superfície $S$ é uma flauta justa (Ver a figura 1.12) quando cada calça $\mathbb{H} / G_{n}$ é uma calça justa.

Observe-se que o conceito de flauta fica bem definido em virtude do Teorema 1.2.24. Os eixos dos geradores $g_{n}, n \geq 0$ (No caso de uma flauta justa o eixo de $g_{0}$ que é parabólico corresponde com um horocíclo, pois a primeira calça tem dois furos) determinam uma sequência aninhada de geodésicas $\left\{\mathcal{E}_{g_{n}}\right\}$ em $\mathbb{H}$ que são projetadas na flauta em curvas geodésicas fechadas que a decompoem em calças. A sequência $\left\{\mathcal{E}_{g_{n}}\right\}$ a sua vez determina um par de sequências $d=\left(d_{n}\right)_{n \geq 1}$ e $\tau=\left(\tau_{n}\right)_{n \geq 1}$, onde $d_{n}$ corresponde à distância entre os eixos $\mathcal{E}_{g_{n-1}}$ e $\mathcal{E}_{g_{n}}$, e $\tau$ corresponde com as coordenadas de torção da sequência aninhada de geodésica $\left\{\mathcal{E}_{g_{n}}\right\}_{n \geq 0}$. Os seguintes são alguns das propriedades das superfícies flautas que aparecem em [Bas93]. Outras propriedades encontram-se em [BK08], [BŠ17], [Evr17], [Haa96], [HS07], [PRT $\left.{ }^{+} 11\right]$, etc.

Proposição 1.4.2. Dada uma sequência de números reais positivos $d=\left(d_{n}\right)_{n \geq 1}$ e uma sequência de números reais $\tau=\left(\tau_{n}\right)_{n \geq 1}$, existe uma superfície flauta com coordenadas $(d, \tau)$.

Proposição 1.4.3. Considere-se uma flauta justa com modelo Fuchsiano G como na definição 1.4.1 e coordenadas $(d, \tau)$.

i) $S e \sum_{n \geq 1} d_{n}=+\infty$, então $G$ é um grupo Fuchsiano do primeiro tipo.

ii) Se $\sum_{n \geq 1} d_{n}<+\infty e \sum_{n \geq 1}\left|\tau_{n}\right|<+\infty$, então $G$ é um grupo Fuchsiano do segundo tipo.

Numa flauta $S$, sejam $\gamma_{n}:=\pi_{G}\left(\mathcal{E}_{g_{n}}\right)$, $\operatorname{logo} \gamma_{n}$ e $\gamma_{n+1}$ são as geodésicas de bordo na calça $\mathbb{H} / G_{n}$, sendo $\gamma_{n}$ a geodésica común com a claça $\mathbb{H} / G_{n-1}$ e $\gamma_{n+1}$ a geodésica común com a calça $\mathbb{H} / G_{n+1}$. $d_{n}$ é por definição a distância hiperbólica entre $\gamma_{n}$ e $\gamma_{n+1}$ como é mostrado na figura 1.14. Em particular quando a flauta seja justa observe-se que $d_{n}=\eta\left(\ell_{s}\left(\gamma_{n}\right)\right)+\eta\left(\ell_{S}\left(\gamma_{n+1}\right)\right)$, ver [Bas90], pelo qual a serie $\sum_{n \geq 1} d_{n}$ diverge se, e somente se, a serie $\sum_{n \geq 1} \eta\left(\ell_{S}\left(\gamma_{n}\right)\right)$ diverge.

Generalizemos agora a definição 1.4 .1 para definir as superfícies gaita de fole. Fixemos em principio um inteiro $m \geq 3$. A ideia é començar com uma esfera $S_{0}$ com $m$ discos fechados disjuntos removidos, para colar depois uma flauta $S^{j}, 1 \leq j \leq m$, em cada buraco de $S_{0}$, ver figura 1.15. Chamaremos de semiflauta justa a uma flauta obtido colando somente calças justas do tipo $(0,1,2)$ (Observe-se que numa flauta justa a primeira calça deve ser do tipo $(0,2,1)$ ).

Definição 1.4.4. Uma superfície gaita de fole de ordem $m \geq 3$ é uma superfície hiperbólica $S$ com modelo Fuchsiano $G=\left\langle G^{0}, G^{1}, \ldots, G^{m}\right\rangle$, onde: 


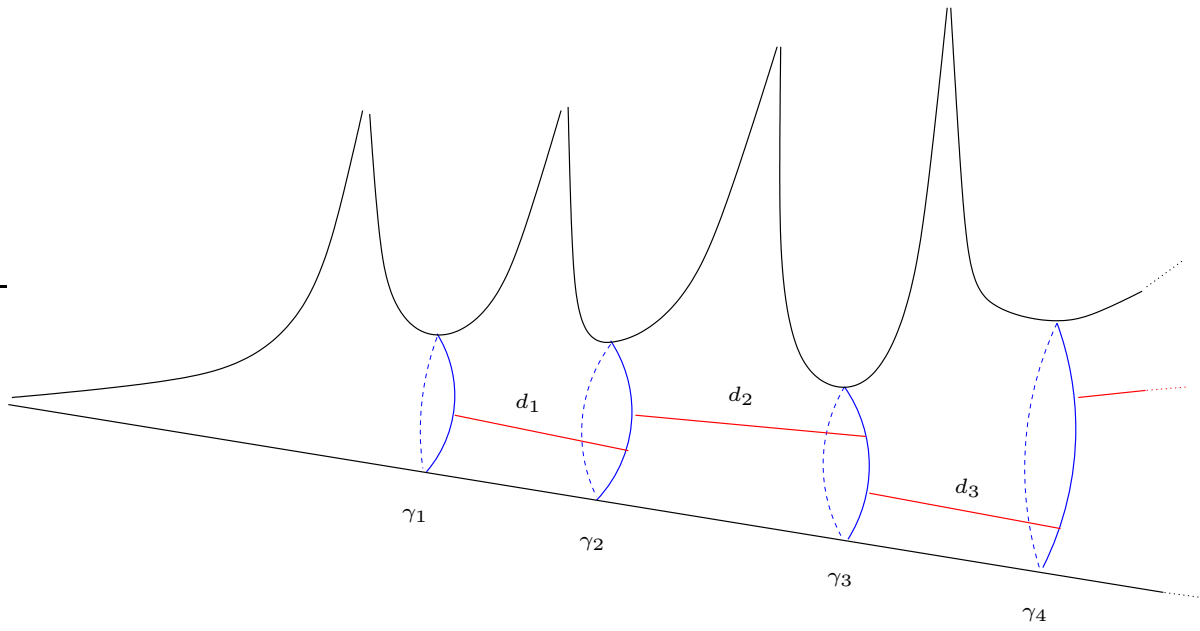

Figura 1.14: Coordenadas $d_{n}$ de uma flauta justa e curvas geodésicas $\gamma_{n}$
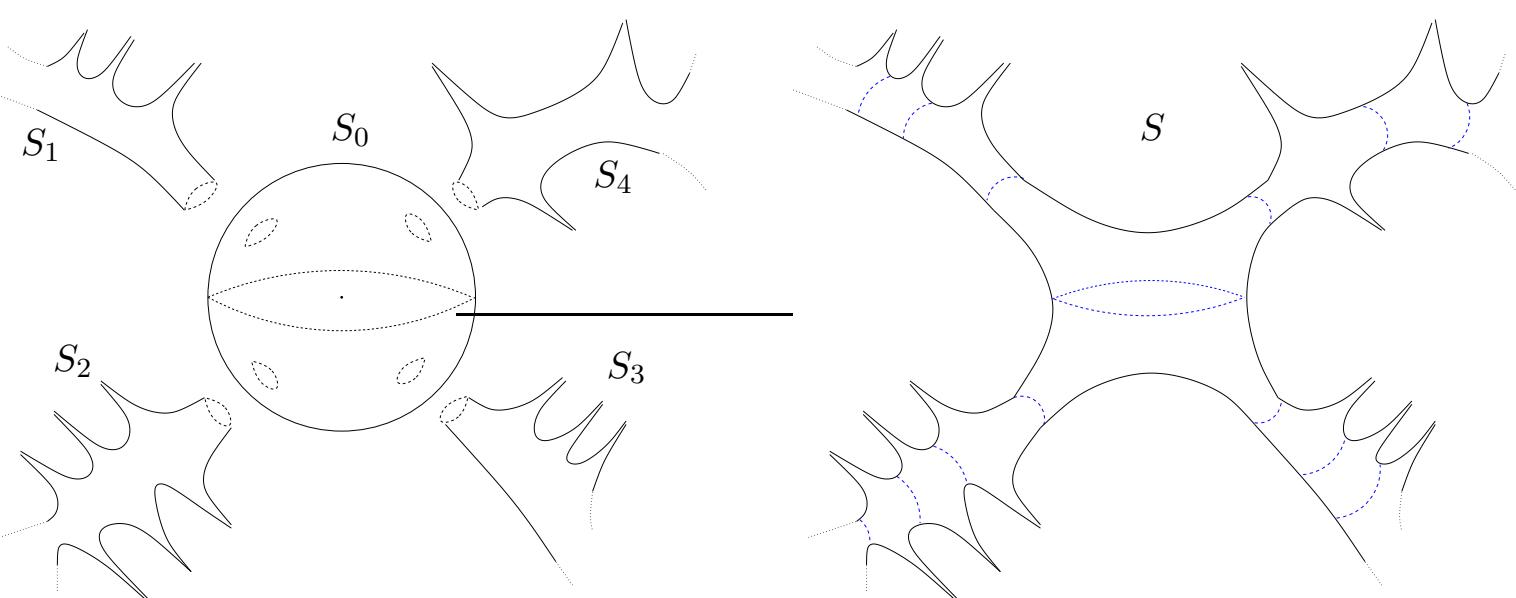

Figura 1.15: Construção de uma gaita de fole com 4 semiflautas justas.

1. $G^{0}$ é o modelo Fuchsiano de uma superfície do tipo conforme $(0,0, m)$ com $m$ elementos hiperbólicos $g_{0}^{1}, g_{0}^{2}, \ldots g_{0}^{m} \in G^{0}$ que satisfazem o seguinte:

i) Se $j_{1} \neq j_{2}$, então $\left\langle g_{0}^{j_{1}}\right\rangle$ e $\left\langle g_{0}^{j_{2}}\right\rangle$ não são $G_{0}$-conjugados.

ii) Se $g \in G_{0}$ é um elemento hiperbólico de bordo de $G_{0}$, então $\langle g\rangle$ e $\left\langle g_{0}^{j}\right\rangle$ são $G_{0}$-conjugados para algum $1 \leq j \leq m$.

2. Para $j=1, \ldots, m, G^{j}=\left\langle G_{n}^{j}\right\rangle_{n=1}^{+\infty}$, sendo cada $G_{n}^{j}$ o modelo Fuchsiano de uma calça com geradores estándar $\left(g_{n-1}^{j}, g_{n}^{j}\right)$, onde cada $g_{n}^{j}$ é um elemento hiperbólico primitivo.

3. $\mathcal{N}\left(G^{0}\right) \cap \mathcal{N}\left(G_{1}^{j}\right)=\mathcal{E}_{g_{0}^{j}}$, para $j=1, \ldots, m$.

4. $\mathcal{N}\left(G_{n}^{j}\right) \cap \mathcal{N}\left(G_{n+1}^{j}\right)=\mathcal{E}_{g_{n}^{j}}$, para todo $n \geq 1$ e $j=1, \ldots, m$.

Se $S^{j}=\mathbb{H} / G^{j}$ é uma semiflauta justa para todo $1 \leq j \leq m$, dize-se que $S$ é uma gaita de fole justa.

Teorema 1.4.5. O grupo $G$ da definição 1.4.4 é Fuchsiano. Além disso, se para cada $k \geq 1 e$ $1 \leq j \leq m, G(k, j)$ e $G(k)$ denotam os grupos:

$$
G(k, j):=\left\langle G_{1}^{j}, G_{2}^{j}, \ldots, G_{k}^{j}\right\rangle,
$$




$$
G(k):=\left\langle G^{0}, G(k, 1), \ldots, G(k, m)\right\rangle
$$

então,

i) $S^{j}$ é uma superfície flauta.

ii) $g_{k}^{1}, g_{k}^{2}, \ldots, g_{k}^{m}$ são elementos hiperbólicos primitivos de bordo de $G(k)$ tais que $\left\langle g_{k}^{j_{1}}\right\rangle$ e $\left\langle g_{k}^{j_{2}}\right\rangle$ não são $G(k)$-conjugados se $j_{1} \neq j_{2}$.

Demonstração. Tome-se $j=1$. Da definição 1.4.4, o grupo $G(j=1):=\left\langle G^{0}, G_{1}^{1}, G_{2}^{1}, G_{3}^{1} \ldots\right\rangle$ satisfaze as hipótese do Teorema 1.2.24, logo é Fuchsiano. Tome-se agora $j=2$ e usando novamente o Teorema 1.2.24 tem-se que o grupo $G(j=1,2):=\left\langle G(j=1), G_{1}^{2}, G_{2}^{2}, G_{3}^{2}, \ldots\right\rangle$ é Fuchsiano. Repitindo o argumento até $j=m$, conclui-se que $G=G(j=1,2, \ldots, m)$ é Fuchsiano. O ítem 2. e o ítem 4. da definição 1.4.4 implicam a afirmação $i$ ).

Considere-se o grupo $\left\langle G^{0}, G_{1}^{1}\right\rangle$. Pelo ítem 3. da definição 1.4.4 tem-se $\mathcal{N}\left(G^{0}\right) \cap \mathcal{N}\left(G_{1}^{1}\right)=\mathcal{E}_{g_{1}^{0}}$, logo pelo Lema 1.2.18 $g_{1}^{1}$ é um elemento de bordo de $\left\langle G^{0}, G_{1}^{1}\right\rangle$. Observe-se que, se $I_{1}^{1}$ é o intervalo de descontinuidade de $G^{0}$ limitado por $\mathcal{E}_{g_{0}^{1}}$ e se $I_{1}^{1}$ é o intervalo de descontinuidade de $G_{1}^{1}$ limitado por $\mathcal{E}_{g_{1}^{1}}$, então $I_{1}^{0} \supset I_{1}^{1}$. Logo, pelo ítem 1. da definição $1.4 .4,\left\langle g_{1}^{1}\right\rangle$ não pode ser $\left\langle G^{0}, G_{1}^{1}\right\rangle$-conjugado como qualquer $\left\langle g_{0}^{j}\right\rangle, j \neq 1$.

Tome-se $\left\langle G^{0}, G_{1}^{1}, G_{1}^{2}\right\rangle$. Como $\mathcal{N}\left(G^{0}\right) \cap \mathcal{N}\left(G_{1}^{2}\right)=\mathcal{E}_{g_{0}^{2}}$, então a convexidade dos núcleos de Nielsen implicam que $\mathcal{N}\left(\left\langle G^{0}, G_{1}^{1}\right\rangle\right) \cap \mathcal{N}\left(G_{1}^{2}\right)=\mathcal{E}_{g_{1}^{2}}$, logo pelo Lema 1.2.18, $g_{1}^{2}$ é um elemento de bordo de $\left\langle G^{0}, G_{1}^{1}, G_{1}^{2}\right\rangle$. Igual que acima, $\left\langle g_{1}^{2}\right\rangle$ não é $\left\langle G^{0}, G_{1}^{1}, G_{1}^{2}\right\rangle$-conjugado com $\left\langle g_{0}^{j}\right\rangle, j \neq 2$ nem com $\left\langle g_{1}^{1}\right\rangle$. Repetindo o argumento e adicionando em cada etapa o grupo $G_{1}^{j}$, conclui-se que $g_{1}^{1}, g_{1}^{2}, \ldots, g_{1}^{m}$ são elementos de bordo de $G(1)=\left\langle G^{0}, G_{1}^{1}, \ldots G_{1}^{m}\right\rangle$, e que os grupos cíclicos gerados por estes elementos não são $G(1)$-conjugados entre sim. Sigue-se a afirmação $i i$ ) ao repetir o argumento acima e usando hipótese de indução nos inteiros $k$.

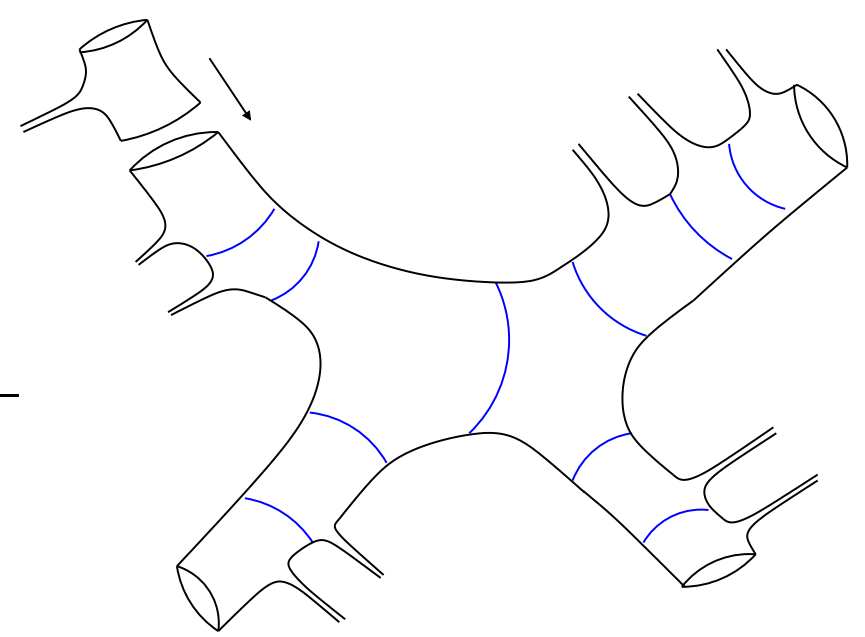

Figura 1.16: Processo de construção da superfície $\mathbb{H} / G(3)$ a partir de $\mathbb{H} / G(2)$.

A proposição anterior, ao garantir que $G$ seja um grupo Fuchsiano, mostra que as superfícies gaita de fole estão bem definidas. Observe-se que do Teorema 1.2.24 tem-se que cada calça $\mathcal{N}\left(G_{n}^{j}\right) / G_{n}^{j}$ está isométricamente mergulhados na superfície $S$, de fato, pode-se ver que cada flauta $S^{j}$ tamém está isométricamnete mergulhada em $S$. Denote-se por $\gamma_{n}^{j}$ a geodésica fechada simples em $S$ dada por $\pi_{G}\left(\mathcal{E}_{g_{n}^{j}}\right), 1 \leq j \leq m, n \geq 0$. Ao tomar $\Gamma$ como a união de $\left\{\gamma_{n}^{j} / 1 \leq j \leq m, n \geq 0\right\}$ e uma multicurva que decompõe em calças a $S_{0}=\mathbb{H} / G^{0}$, obtemos uma multicurva que decompõe em calças a superfície gaita de fole $S$. Por outro lado, sendo cada $S^{j}$ uma flauta, ésta tem associada um par de coordenadas $d^{j}=\left(d_{n}^{j}\right)_{n \geq 0}$ e $\tau^{j}=\left(\tau_{n}^{j}\right)_{n \geq 0}$ onde $d_{n}^{j}>0$ e $\tau_{n}^{j} \in \mathbb{R}$, para todo $j, n$. 
Procuremos à continuação generalizar a Proposição 1.4.3 para os casos das superfícies gaita de fole de ordem $m \geq 3$.

Teorema 1.4.6. Seja $S$ uma gaita de fole justa de ordem $m$ com modelo Fuchsiano $G=\left\langle G^{0}, G^{1}, \ldots, G^{m}\right\rangle$ como na definição 1.4.4, e com coordenadas $\left(d^{1}, d^{2}, \ldots, d^{m}, \tau^{1}, \tau^{2}, \ldots, \tau^{m}\right)$.

i) Se para cada $1 \leq j \leq m, \sum_{n=0}^{+\infty} d_{n}^{j}$ diverge, então $G$ é um grupo Fuchsiano do primeiro tipo.

ii) Se para algum $1 \leq j \leq m, \sum_{n=0}^{+\infty} d_{n}^{j}$ converge e $\sum_{n=0}^{+\infty}\left|\tau_{n}^{j}\right|$ converge, então G é um grupo Fuchsiano do segundo tipo.

Antes de fazer a demostração da proposição acima, precisaremos dos dois seguintes lemas que são a versão para gaitas do lema 1 e 2 da seção 6 de [Bas93].

Lema 1.4.7. Com as notações anteriores suponha que para algum $1 \leq j \leq m,\left\{\mathcal{E}_{h_{n}^{j}}\right\}_{n \geq 0}$, sendo cada $h_{n}^{j} \in \mathrm{PSL}_{2}(\mathbb{R})$ um elemento hiperbólico, é uma sequência aninhada de geodésicas que converge à geodésica $L$, onde para cada $n \geq 0,\left\langle h_{n}^{j}\right\rangle$ é $G(n)$-conjugado com $\left\langle g_{n}^{j}\right\rangle$. Se $I \subset \mathbb{R}$ é o intervalo aberto limitado pela geodésica L, então I é um intervalo de descontinuidade de G.

Demonstração. É suficiente provar que $I$ é precisamente invariante pela identidade em $G$ pois sendo as extremidades no infinito de cada geodésica $\mathcal{E}_{h_{n}^{j}}$ pontos limites de $\Lambda_{G}$, pelo Lema 1.2 .15 , I deve ser intervalo de descontinuidade do grupo $G$.

Seja $g \in G, g \neq \mathrm{Id}$. Tomando $n$ suficientemente grande pode-se assumir sem perda de generalidade que $g \in G(n) \backslash \bigcup_{f \in G(n)} f \cdot\left\langle g_{n}^{j}\right\rangle \cdot f^{-1}$. Como $\left\langle g_{n}^{j}\right\rangle$ é $G(n)$-conjugado à $\left\langle h_{n}^{j}\right\rangle$, então o intervalo $I_{n}$ limitado por $\mathcal{E}_{h_{n}^{j}}$ é um intervalo de descontinuidade de $G(n)$. Assim, pela Proposição 1.2.14, $I_{n}$ é precisamente invariante por $\left\langle h_{n}^{j}\right\rangle$ em $G(n)$. Como $g \notin\left\langle h_{n}^{j}\right\rangle$, então $g I_{n} \cap I_{n}=\emptyset$. Portanto $g I \cap I=\emptyset$ pois $I \subset I_{n}$.

Lema 1.4.8. Com as notações anteriores, se I é um intervalo de descontinuidade de $G$, então ou I é limitado pelo eixo de um elemento hiperbólico de bordo de $G$, ou I está limitado por uma geodésica limite de uma sequência aninhada de eixos $\left\{\mathcal{E}_{h_{n}^{j}}\right\}_{n \geq 1}$ para algum $1 \leq j \leq m$, onde $\left\langle h_{n}^{j}\right\rangle$ é $G^{j}$-conjugado à $\left\langle g_{n}^{j}\right\rangle$ para todo $n \geq 0$.

Demonstração. Suponha que $I$ não é o intervalo de descontinuidade de um elemento hiperbólico de bordo de $G$. Como $G(n)<G$ para todo $k \geq 0$, então pela observação $1.2 .17 i i), I$ está contido num intervalo de descontinuidade de $G(n)$ o qual, pelo Teorema $1.4 .5 \mathrm{ii}$ ) deve ser $G(n)$-conjugado à algum $\left\langle g_{n}^{j}\right\rangle$, sendo o superíndice $j=1, \ldots, m$ o mesmo. Assim, existem intervalos $I_{n}$ tais que $I \subsetneq I_{n}$, sendo $I_{n}$ um intervalo de descontinuidade de $G(n)$ limitado pelo eixo $h_{n}^{j}$, onde $\left\langle h_{n}^{j}\right\rangle$ é $G(n)$-conjugado com $\left\langle g_{n}^{j}\right\rangle$. Como $G(n)<G(n+1)$, então $I_{n+1} \subsetneq I_{n}$. Por outro lado $\mathcal{E}_{h_{n}^{j}}$ e $\mathcal{E}_{h_{n+1}^{j}}$ não tem uma extremidade común (Ver Lema 2.20 em [YT92]). Em consequência a sequência de eixos acima é em efeito uma sequência aninhada e, além disso, existe uma linha geodésica $L$ que limita o intervalo aberto $I^{\prime}=\bigcap_{n \geq 1} I_{n} \supset I$ tal que a sequência aninhada $\left\{\mathcal{E}_{h_{n}^{j}}\right\}_{n \geq 0}$ converge à $L$. Pelo Lema 1.4.7, $I^{\prime}$ é um intervalo de descontinuidade de $G$, mas como $I$ também é intervalo de descontinuidade tem-se necessariamente que $I=I^{\prime}$. 
Demostração da Proposição 1.4.7. i). Suponha que $G$ é do segundo tipo e seja $I \subset \hat{\mathbb{R}}$ um intervalo de descontinuidade de $G$. Como $S$ é uma gaita justa, $G$ não têm elementos hiperbólicos de bordo, logo pelo Lema 1.4 .8 a geodésica $L$ que limita à $I$ é o limite de uma sequência aninhada de eixos $\left\{\mathcal{E}_{h_{n}^{j}}\right\}_{n \geq 0}$ para algum $1 \leq j \leq m$, sendo $\left\langle h_{n}^{j}\right\rangle G^{j}$-conjugado com $\left\langle g_{n}^{j}\right\rangle$. Observe-se que $d_{n}^{j} \leq d_{\mathbb{H}}\left(\mathcal{E}_{h_{n}^{j}}, \mathcal{E}_{h_{n+1}^{j}}\right)$ para todo $n \geq 0$. Assim, pela Proposição 1.2.4, $\sum_{n \geq 0} d_{n}^{j}<+\infty$.

ii). Suponha que $\sum_{n \geq 0} d_{n}^{j}<+\infty$ e $\sum_{n \geq 0}\left|\tau_{n}^{j}\right|<+\infty$ para algum $1 \leq j \leq m$. Pela Proposição 1.2 .5 a sequência aninhada $\left\{\mathcal{E}_{g_{n}^{j}}\right\}_{n \geq 0}$ converge a uma geodésica $L$ que limita um intervalo aberto $I \subset \hat{\mathbb{R}}$. Pelo Lema 1.4.7 conclui-se que $G$ é do segundo tipo.

Definição 1.4.9. Uma superfície gaita de fole de ordem 1 é uma flauta. Uma superfície gaita de fole de ordem 2 é uma superfície $S$ com modelo Fuchsiano $G=\left\langle G^{0}, G^{1}, G^{2}\right\rangle$, como na definição 1.4.4, onde o ítem 1. é substituído pelo seguinte:

1'. $G^{0}$ é o modelo Fuchsiano de uma calça do tipo $(0,1,2)$.

Se as calças $S^{j}=\mathbb{H} / G^{j}, j=1,2$, são todas justas, dize-se que a gaita é justa.

O Teorema 1.4.5 e a Proposição 1.4.6 valem também para uma gaita de fole de ordem 2. A figura 1.17 mostra o desenho de uma 2-gaita de fole a qual também podem chamar-lhe de biflauta.

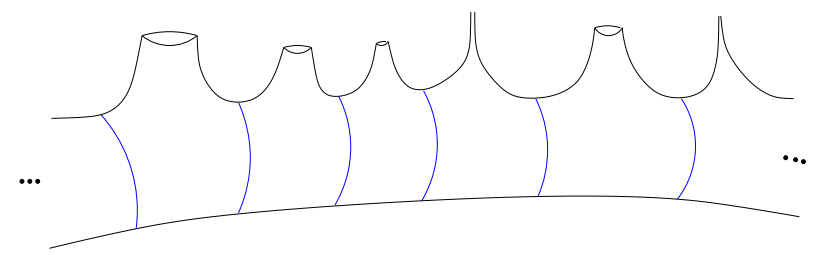

Figura 1.17: Superfície gaita de fole de ordem 2 ou biflauta

Para finalizar vamos a considerar uma caracterização alternativa e de fato mais simple para as superfícies gaita de fole. A caracterização é baseada no conceito de fin de uma superfície e foi dada por A. Haas e P. Susskind para o caso das superfćies flauta, ver [HS07]. Começamos com a definição do espaços dos fins. Considere-se uma superfície de Riemann $S$ (A verdade basta que $S$ seja um espaço hemicompacto) e uma sequência crescente de compactos $K_{1} \subset K_{2} \subset \ldots$ tais que $\bigcup_{n \geq 1} K_{n}=S$; chama-se de exaustão à sequência $\left\{K_{n}\right\}_{n \geq 1}$. Seja $E_{1} \supset E_{2} \supset \ldots$, uma sequência decrescente de subconjuntos de $S$, sendo cada $E_{n}$ uma componente conexa de $S \backslash K_{n}$. Denote-se por $\mathcal{F}_{0}(S)$ a coleção de sequências $\left\{E_{n}\right\}_{n \geq 1}$ como acima para alguma exaustão $\left\{K_{n}\right\}_{n \geq 1}$ de $S$ e considere-se a seguinte relação de equivalência em $\mathcal{F}_{0}(S):\left\{E_{n}\right\}_{n \geq 1} \sim\left\{E_{n}^{\prime}\right\}_{n \geq 1}$ se existem $N \geq 1$ e $k \geq 0$ tal que $E_{n+k} \subset E_{n}^{\prime}, n \geq k$, e vice-versa.

Definição 1.4.10. O espaço dos fins da superfície $S$ define-se como o quociente $\mathcal{F}(S):=\mathcal{F}_{0}(S) / \sim$, dotado da topología induzida pela seguinte coleção de vizinhanças: Dado um conjunto aberto $U \subset S$, $U^{*}$ consiste em todos os fins $\left[E_{n}\right]_{n \geq 1}$ tais que $E_{n} \subset U$ para $n$ grande. Um fin $\left[E_{n}\right]_{n \geq 1}$ é um furo se para algum representante $\left\{E_{n}\right\}_{n \geq 1}$ existe $E_{N}$ conformemente equivalente a um disco furado; dize-se em cambio que é um buraco se para algum representante $\left\{E_{n}\right\}_{n \geq 1}$ existe $N \geq 1$ tal que $E_{n}$ é um anel de módulo finito para todo $n \geq N$. Um fin chama-se do tipo finito se é um furo ou um buraco, caso contrario chama-se do tipo infinito. 
Proposição 1.4.11. S é uma superfície gaita de fole de ordem $m$ se, e somente se, $S$ é conformemente equivalente a um dominio de $\hat{\mathbb{C}}$ com $m$ fins infinitos.

Demonstração. A implicação direta é consequência do Teorema de planaridade de Koebe pois numa superfície gaita toda curva fechada simples separa à superfície em dois componentes conexas. Suponha-se que $S$ é conformemente equivalente a um dominio de $\widehat{\mathbb{C}}$ com $m$ fins infinitos; suponha por simplicidade que $m \geq 3$. Não há perda de generalidade em supor que $S=\hat{\mathbb{C}} \backslash X$, onde $X$ é um subespaço fechado de $\widehat{\mathbb{C}}$. Por outro lado, em qualquer superfície, o espaço dos fins é homeomorfo a um subespaço fechado de um conjunto de Cantor, assim que no caso da superfície $S$ que têm $m$ fins infinitos, estos correspondem com $m$ componentes $X^{1}, \ldots X^{m}$ de $X$ nos quais acumulan-se o resto das componentes de $X$ que a sua vez correspondem com fins de $S$ do tipo finito (Logo são pontos se o fin é um furo e são continuos não triviais se o fin é um buraco). Usando uma versão fraca da Proposição 1.3.8 pode-se escolher $m$ geodésicas fechadas simples $\gamma_{0}^{1}, \ldots, \gamma_{0}^{m} \subset S$ que separem à $\hat{\mathbb{C}}$ em $m+1$ componentes onde $X^{j}$ e infinitas componentes de $X$ que se acumulam neste fin do tipo infinito estão contidos no disco limitado por $\gamma_{0}^{j}, 1 \leq j \leq m$ e, uma única componente de $\hat{\mathbb{C}} \backslash \cup_{j} \gamma_{0}^{j}$ que não contém o conjunto $X$; observe-se que esta última componente é um dominio conformemente equivalente a uma esfera com $m$ buracos, enquanto que as outras $m$ componentes são conformemente equivalentes a um dominio de um disco aberto com um único fin infinito.

Seja $U^{j}, 1 \leq j \leq m$, a componente de $S \backslash \cup_{1 \leq j \leq m} \gamma_{0}^{j}$ que contém à $X^{j}$. Para finalizar a prova basta mostrar que cada $U^{j}$ é uma flauta; isto segue-se da Proposição 1 do [HS07], porém será reproduzida a prova para fazer o texto o mais auto-contido possível. Os fins finitos de $X$ "contidos"em $U^{j}$ podemse ordenar como uma sequência $\left\{X_{n}^{j}\right\}_{n \geq 1}$, por exemplo tomando $d_{\widehat{\mathbb{C}}}\left(X_{n}^{j}, X^{j}\right) \geq d_{\widehat{\mathbb{C}}}\left(X_{n+1}^{j}, X^{j}\right)$, de forma que na topología de $\mathcal{F}(S)$ tenha-se que $X_{n}^{j} \longrightarrow X^{j}$. Ao tomar para cada $n \geq 1$, uma geodésica $\gamma_{n}^{j} \subset S$ fechada e simples tal que a componente de $U^{j} \backslash \gamma_{n}^{j}$ que contenha à $X^{j}$ contenha também à $X_{k}^{j}$ para todo $k>n$, em quanto que a outra componente contenha os fechados $X_{1}^{j}, \ldots, X_{n}^{j}$, construyese uma sequência $\left\{\gamma_{n}^{j}\right\}_{n \geq 1}$ que separa em calças à $U^{j}$. Daí cada dominio $U^{j}$ é obtido colando consecutivamente uma sequência de calças a través das geodesicas $\gamma_{n}^{j}$, logo $U^{j}$ é uma flauta.

Como comentário final observe-se que as superfícies gaita de fole podem-se generalizar um pouco mais pedindo-lhes que a superfície $S_{0}=\mathbb{H} / G^{0}$ tenha gênero positivo com o qual teriam-se superfícies gaitas de ordem $m$ e gênero $g$. Acredito que as proposições anteriores valeríam também neste caso. 


\section{Capítulo 2}

\section{Espaços de papel}

Neste capítulo serão definidos os espaços chamados de papel fazendo úso do conceito de quociente métrico da seção 1.1 do capítulo anterior, e serão mostrados alguns exemplos e propriedades básicas de estos espaços. As seções 2.2 e 2.3 são tomadas do [dCH12], [Ber12], e a seção 2.1 essencialmente é tomado de [dC05] e [CGL06]. Convido ao leitor a dar uma olhada a estas seções para comprender o conceito de espaço de papel, os exemplos e os tipos de pontos nele sem entrar nos detalhes se quiser. A seção 2.4, principalmente o Teorema 2.4.4 será importante para o seguinte capítulo.

\subsection{Ferradura justa}

A ferradura justa é uma tranformação definida sobre uma esfera topológica que reproduz a ação da conhecida ferradura de Smale com a diferença de que a ferradura justa não possui pontos com dinâmica irrelevante: $\mathrm{O}$ conjunto não-errante desta aplicação é a superfície toda. A ferradura justa é o exemplo mais simple de uma classe de homeomorfismos sobre superfícies chamados de pseudo-Anosov generalizados; como seu nome indica, estos generalizam o concito clássico de homeomorfismos pseudo-Anosov mas, neste caso, são permitidos um número infinito de singularidades, só que estos acumulan-se num número finito de pontos da superfície. A definição formal de homeomorfismo pseudo-anosov generalizado será a seguinte.

Definição 2.1.1. Seja $f: S \longrightarrow S$ um homeomorfismo definido sobre uma superfície diferenciável $S$. Dize-se que $f$ é um homeomorfismo pseudo-Anosov generalizado se existem:

a) Um conjunto finito $Q \subset S$ invariante por $f$;

b) Um par de folheações mensuradas transversais $\left(\mathcal{F}^{s}, \mu^{s}\right)$ e $\left(\mathcal{F}^{u}, \mu^{u}\right)$ de $S \backslash Q$ com uma quantidade enumerável de singularidades de grau $n \geq 1$ (dos tipos mostrados na figura 2.1) os quais acumulan-se somente nos pontos de $Q$;
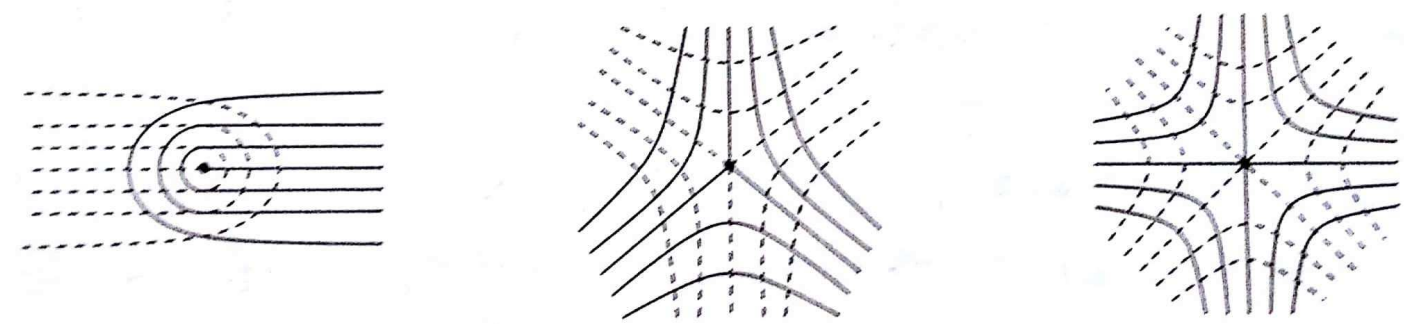

Figura 2.1: Singularidades de grau 1, 3 e 4 admitidas nas folheações de um pseudo-Anosov generalizado 
c) Um número real $\lambda>1$;

Tais que,

$$
\begin{aligned}
& f\left(\mathcal{F}^{s}, \mu^{s}\right)=\left(\mathcal{F}^{s}, \frac{1}{\lambda} \cdot \mu^{s}\right) \\
& f\left(\mathcal{F}^{u}, \mu^{u}\right)=\left(\mathcal{F}^{u}, \lambda \cdot \mu^{u}\right)
\end{aligned}
$$

Em particular o homeomorfismo $f$ será um pseudo-Anosov no sentido tradicional quando tenha um número finito de singularidades ou, equivalentemente, quando $Q=\emptyset$.

Este capítulo comença com a descrição da ferradura justa e principalmente da superfície onde tal aplicação é definida: este corresponde com uma esfera de papel com pelo menos um ponto irregular os quais são protagonistas importantes nesta tese. Assim começamos com um primeiro exemplo interesante de um espaço de papel que espero sirva de motivação para o resto do conteúdo das seções a seguir.

Considere-se o quadrado unitario $P=\{x+i y / 0 \leq x, y \leq 1\}$ e a aplicação $\hat{f}: P \longrightarrow P$ definida, para todo $z=x+i y \in P$, por,

$$
\hat{f}(z):=\left\{\begin{array}{ccc}
2 x+i \frac{y}{2} ; & \text { se } & x \leq \frac{1}{2} \\
2(1-x)+i\left(1-\frac{y}{2}\right) ; & \text { se } & x>\frac{1}{2}
\end{array}\right.
$$

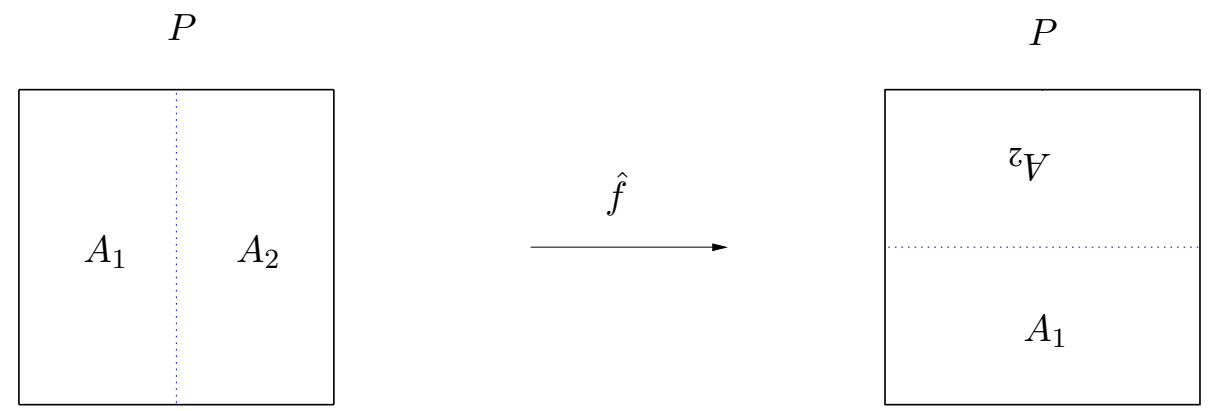

Figura 2.2: Ação de $\hat{f}$ sobre o quadrado.

No desenho acima mostra-se a ação de $\hat{f}$ sobre $P: \hat{f}$ contrai verticalmente por um fator $\frac{1}{2}$, e expande horizontalmente por um fator igual a 2 , dobrando a metade direita $A_{2}$ de $P$ justamente acima da metade esquerda $A_{1}$. Claramente $\hat{f}$ não é uma aplicação injetora nem sobrejetora e pior aínda, $\hat{f}$ não é continua pois pontos a ambos lados do segmento vertical central $\operatorname{Re} z=\frac{1}{2}$ são considerablemente separados entre sim. A ideia será fazer identificações adequadas de pares de subsegmentos de igual comprimento contidos na fronteira do quadrado para convertir a aplicação $\hat{f}$ num homeomorfismo.

Para corrigir a descontinuidade de $\hat{f}$ observe-se que o lado direito de $A_{1}$ é levado pela $\hat{f}$ no subsegmento $\alpha_{-1}=\left\{1+i y / 0 \leq y \leq \frac{1}{2}\right\}$, enquanto que o lado esquerdo de $A_{2}$ e levado pela $\hat{f}$ no subsegmento $\alpha_{-1}^{\prime}=\left\{1+i y / \frac{1}{2} \leq y \leq 1\right\}$ mas invertendo este segmento. Ao identificar cada ponto $1+i\left(\frac{1}{2}-t\right) \in \alpha_{-1}$ com $1+i\left(\frac{1}{2}+t\right) \in \alpha_{-1}^{\prime}$, para $0 \leq t \leq \frac{1}{2}$, a aplicação $\hat{f}$ fica continua no segmento $\operatorname{Re} z=\frac{1}{2}$; a figura 2.3 na esquerda mostra a identificação descrita. Observe-se que o ponto 

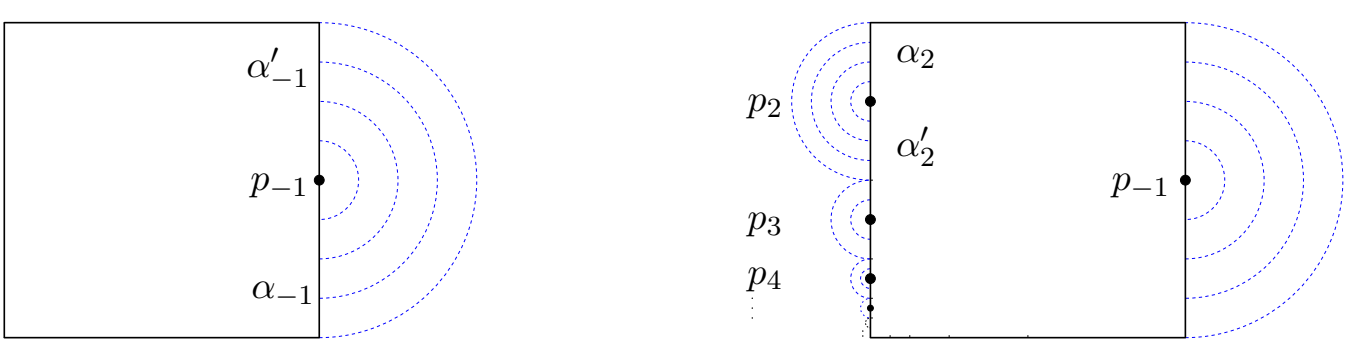

Figura 2.3: Identificação dos lados verticais do quadrado.

$\hat{p}_{-1}=1+\frac{i}{2}$ é o único que é identificado com ele mesmo; este tipo de ponto também é chamado de ponto de dobradura por rações obvias.

Definindo para cada $n \geq 1$ os pares de subsegmentos $\alpha_{n+1}:=\hat{f}^{n}\left(\alpha_{-1}\right)$ e $\alpha_{n+1}^{\prime}:=\hat{f}^{n}\left(\alpha_{-1}^{\prime}\right)$, a identificação feita no lado direito do quadrado deve ser reproduzido na união de todos estos pares de forma que a aplicação fique bem definida, ver o outro desenho na figura ??. Assim o segmento $\alpha_{n+1}$ é dobrado acima no segmento $\alpha_{n+1}^{\prime}$, sendo o ponto meio $\hat{p}_{n+1}$ de $\alpha_{n+1} \cup \alpha_{n+1}^{\prime}$ um novo ponto de dobradura. Com as identificações anteriores a aplicação $\hat{f}$ vira numa transformação continua.

Para fazer agora que a aplicação seja injetora observe-se que tanto o subsegmento $\alpha_{1}=\{x+$ $\left.i / 0 \leq x \leq \frac{1}{2}\right\}$ como o subsegmento $\alpha_{1}^{\prime}=\left\{x+i / \frac{1}{2} \leq x \leq 1\right\}$ são mapeados no mesmo segmento horizontal $\operatorname{Im} z=\frac{1}{2}$ de $P$, sendo o segundo subsegmento virado acima do segmento. Dobrando o lado superior do $P$ pela mitade e identificando o ponto $\left(\frac{1}{2}-t\right)+i \in \alpha_{1}$ com o ponto $\left(\frac{1}{2}+t\right)+i \in \alpha_{1}^{\prime}$, para cada $0 \leq t \leq \frac{1}{2}$, fazemos que a aplicação seja injetora no lado superior de $P$; denote-se por $\hat{p}_{1}$ o ponto de dobradura ao fazer a identificação anterior. Como o lado inferior de $A_{2}$ é transformado no lado superior de $P$, para preservar a injetividade precisa-se dividir em dois partes iguais este subsegmento e identificar ambas metades. Específicamente cada ponto $\frac{3}{4}-t$ no subsegmento $\alpha_{-2}^{\prime}=\hat{f}^{-1}\left(\alpha_{1}^{\prime}\right)$ é identificado com o ponto $\frac{3}{4}+t$ no subsegmento $\alpha_{-2}=\hat{f}^{-1}\left(\alpha_{1}\right)$, para $0 \leq t \leq \frac{1}{4}$; denota-se por $\hat{p}_{-2}$ ao ponto de dobradura de esta identificação, é dizer, $\hat{p}_{-2}=\frac{3}{4}$. Repetindo este processo para cada $n \geq 2$, definindo $\alpha_{-(n+1)}=\hat{f}^{-n}\left(\alpha_{1}\right)$ e $\alpha_{-(n+1)}^{\prime}=\hat{f}^{-n}\left(\alpha_{1}^{\prime}\right)$, e dobrando cada um destos subsegmentos pelo ponto de dobradura $\hat{p}_{-(n+1)}=\frac{3}{2^{n+1}}$, consegue-se fazer de $\hat{f}$ uma aplicação injetora em todos os pontos. A coleção de pares de intervalos $\alpha_{n}^{\prime} \cup \alpha_{n}, n \in \mathbb{Z}^{*}$ cobrem a fronteira de $P$ exceto no vértice 0 . A figura 2.4 mostra o quadrado $P$ junto com todas as identificações descritas anteriormente.

A união de todas as relações de equivalências definidas em cada $\alpha_{n}^{\prime} \cup \alpha_{n}$ determina uma relação $\mathcal{P}$ em $P$ simétrica, reflexiva más não transitiva. Porém, ao considerar o quociente métrico $S=P / d_{P}^{\mathcal{P}}$, sendo $d_{P}$ a métrica Euclideana intrínsica no quadrado, a aplicação $\hat{f}$ projeta-se num homeomorfismo $f: S \longrightarrow S$ que chamaremos de ferradura justa. Se $\pi_{\mathcal{P}}$ denota a aplicação projeção sobre este quociente, é fácil ver que $q=\pi_{\mathcal{P}}(0)$ é o único ponto com infinitas $\pi_{\mathcal{P}}$-preimagems e além disso é o ponto de acumulação de todos os pontos de dobraduras $p_{n}=\pi_{\mathcal{P}}\left(\hat{p}_{n}\right), n \neq 0$. De fato $q$ é um ponto fixo de $f$ e o conjunto $\left\{p_{n}\right\}_{n \in \mathbb{Z}^{*}}$ é a órbita de qualquer de seus pontos, a qual no futuro e no pasado converge para o ponto $q$ relativo a dinámica dada pelo homeomorfismo $f$. A figura 2.5 mostra a ação de $f$ sobre o quociente $S$.

Com relação as propriedade geométricas do $S$, este é homeomorfo a uma esfera e admite uma estrutura complexa, logo é biholomorfo à $\widehat{\mathbb{C}}$, ver [dC05] para mais detalhes. As folheações vertical e horizontal do quadrado $P$ são projetados sobre $S$ num par de folheações mensuradas transversais $\left(\mathcal{F}^{s}, \mu^{s}\right)$ e $\left(\mathcal{F}^{u}, \mu^{u}\right)$ respectivamente, ambas invariantes pelo $f$; A aplicação $f$ expande por um fator $\lambda=2$ na folheação horizontal e contrai por um fator $\frac{1}{2}$ a folheação vertical. Os pontos de dobraduras $p_{n}$ correspondem com as singularidades de ambas folheações, sendo todas estas de grau 1. Assim conclui-se que a ferradura justa é um homeomorfismo pseudo-Anosov generalizado como na definição 2.1.1, onde $Q=\{q\}$. O exemplo acima desenvolvido é uma simplificação de uma receita 


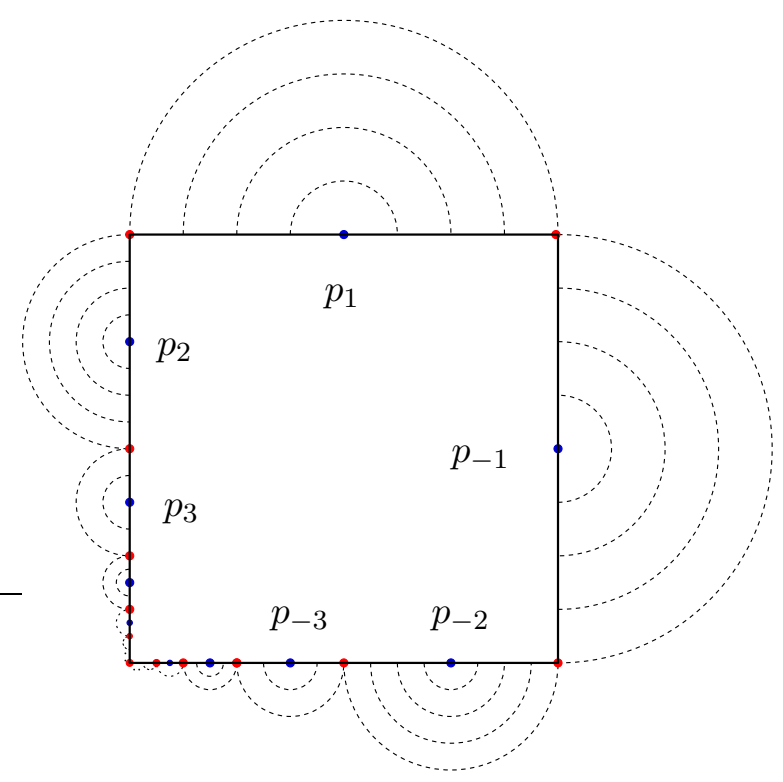

Figura 2.4: $O$ quadrado $P$ com todas as identificações e os pontos de dobradura.
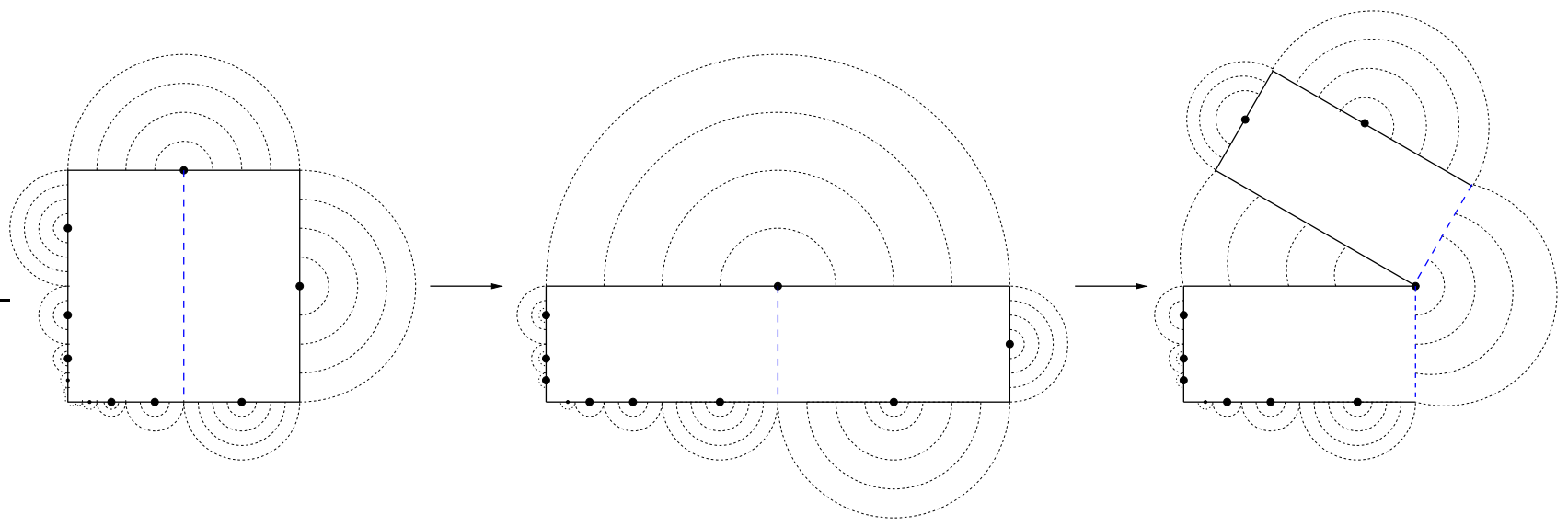

Figura 2.5: Ação de $f$ sobre a superfície $S$.

mais geral feita em [dC05] (ver também [dCH04], [dCH10]), que permite construir homeomorfismos pseudo-Anosov generalizados definidos sobres espaços de papel, a partir de uma classe especial de aplicações chamadas de homeomorfismos sobre grafos grossos. O leitor interesado pode consultar as refencias citadas para conhecer os detalhes desta receita.

\subsection{Espaços de papel}

Um arco num espaço métrico $X$ é uma imagem homeomorfa $\gamma \subset X$ do intervalo [0,1]. Seus pontos extremos são as imagens de 0 e 1 e seu interior, denotado por $\dot{\gamma}$, é a imagem de $(0,1)$. Uma curva fechada simple em $X$ é a imagem homeomorfa de um círculo. Um segmento é um arco em $\mathbb{C}$ o qual está contido numa linha reta. O comprimento de um segmento $\alpha$ denota-se $|\alpha|$. Um arco ou curva fechada simples em $\mathbb{C}$ chama-se de poligonal se ela é a concatenção de um número finito de segmentos; cada segmento maximal numa poligonal chama-se de lado, e a interseção de dois lados consecutivos de uma poligonal chama-se de vértice.

Um polígono é um disco topológico fechado em $\mathbb{C}$, cuja fronteira é uma poligonal fechada simples. Um multipolígono é uma união finita e disjunta de polígonos em $\mathbb{C}$. Os vértices e lados de um 
multipolígono são os respectivos vértices e lados de sua fronteira. Num multipolígono $P$ sempre será considerada a métrica intrínseca induzida pela métrica euclideana $d_{\mathbb{C}}$, a qual será denotada por $d$ em vez de $d_{P}$ desde que não exista perigo de confusão. Observe-se que a topología induzida por $d_{P}$ é a mesma que a topología de subespaço de $P$ (Observação 1.1.4, ii)), logo $\left(P, d_{P}\right)$ é um espaço métrico compacto. Num multipolígono, sempre será fixada a orientação anti-horaria na fronteira de cada um do seus polígonos. Para cada ponto $x \in \partial P$, denota-se por $\xi_{P}(x)$ ao ângulo interno de $P$ em $x$; se $x$ está no interior do multipolígono $P$, então $\xi_{P}(x):=2 \pi$.

Definição 2.2.1. Seja $P \subset \mathbb{C}$ um multipolígono e $\alpha, \alpha^{\prime} \subset \partial P$ dois segmentos de igual comprimento com interiores disjuntos (não necessariamente maximales). O pareamento de $\alpha$ e $\alpha^{\prime}$, denotado $\left\langle\alpha, \alpha^{\prime}\right\rangle$, é a relação que identifica pares de pontos de $\alpha$ e $\alpha^{\prime}$ por uma isometría $\alpha \longrightarrow \alpha^{\prime}$ que reverte orientação. Os segmentos $\alpha$ e $\alpha^{\prime}$ e quaisquer par de pontos identificados por $\left\langle\alpha, \alpha^{\prime}\right\rangle$ são ditos pareados. O comprimento de um pareamento, denotado por $\left|\left\langle\alpha, \alpha^{\prime}\right\rangle\right|$, é o comprimento común dos segmentos pareados $|\alpha|=\left|\alpha^{\prime}\right|$.

Uma coleção $\left\{\left\langle\alpha_{i}, \alpha_{i}^{\prime}\right\rangle\right\}_{i}$ de pareamentos em $P$ são interiormente disjuntas se o conjunto $\left\{\stackrel{\circ}{\alpha}_{i}, \stackrel{\circ}{\alpha}_{i}^{\prime}\right\}_{i}$ são disjuntos dois a dois. Se $\mathcal{P}=\left\{\left\langle\alpha_{i}, \alpha_{i}^{\prime}\right\rangle\right\}_{i}$ é uma união enumerável de pareamentos, o qual é interiormente disjunto, seu comprimento, denotado por $|\mathcal{P}|$, é a soma dos comprimentos de seus pareamentos. Neste caso dize-se que $\mathcal{P}$ é total, se $|\mathcal{P}|$ for igual a metade do perímetro de $\partial P$.

Quando $\mathcal{P}\left\{\left\langle\alpha_{i}, \alpha_{i}^{\prime}\right\rangle\right\}_{i}$ é total e interiormente disjunto, a união $\cup_{i}\left(\alpha_{i} \cup \alpha_{i}^{\prime}\right)$ cobre $\partial P$ a menos de um conjunto de medida de Lebesgue 1-dimensional igual à 0 . Uma coleção de pareamentos $\mathcal{P}$ num multipolígono $P$ determina uma relação em $P$ reflexiva e simétrica chamada relação de pareamento a qual será denotada igualmente por $\mathcal{P}$, onde para todo par de pontos $x, x^{\prime} \in P,\left(x, x^{\prime}\right) \in \mathcal{P}$ se $x=x^{\prime}$ ou $x, x^{\prime}$ são pareados. Observe-se que em geral a relação de pareamento não é transitiva e que todo ponto em $P$ está $\mathcal{P}$-relacionado com a lo más dois pontos diferentes.

Definição 2.2.2 (Espaço de papel). Uma dobradura de papel, ou simplemente dobradura, é um par $(P, \mathcal{P})$, onde $P$ é um multipolígono e $\mathcal{P}$ é uma coleção de pareamentos interiormente disjunto e total de $\partial P$. O quociente métrico $S_{\mathcal{P}}:=P / d_{P}^{\mathcal{P}}$ chama-se de espaço de papel associado a dobradura de papel $(P, \mathcal{P})$.

Observe-se que ao ser $(P, d)$ um espaço métrico compacto, o quociente métrico $P / d_{P}^{P}$ é homeomorfo ao quociente topológico $P / \sim_{\mathcal{P}}$ (proposição 1.1.10), logo o espaço de papel $\left(S_{\mathcal{P}}, d^{\mathcal{P}}\right)$ sempre será um espaço métrico compacto. Além disso e como conseqüência das proposições 1.1.11 e 1.1.6, a métrica $d^{\mathcal{P}}$ é estritamente intínseca em $S_{\mathcal{P}}$.

Denote-se por $\pi_{\mathcal{P}}: P \longrightarrow S_{\mathcal{P}}$ a $\sim_{\mathcal{P}}$-projeção do multipolígono $P$ sobre o espaço de papel $S_{\mathcal{P}}$. O subespaço $G_{\mathcal{P}}:=\pi_{\mathcal{P}}(\partial P)$ chama-se de cicatriz do $S_{\mathcal{P}}$; observe-se que $\pi_{\mathcal{P}}$ : int $P \longrightarrow S_{\mathcal{P}} \backslash G_{\mathcal{P}}$ é um homeomorfismo. Sobre a cicatriz $G_{\mathcal{P}}$ de um espaço de papel $S_{\mathcal{P}}$ será considerada a métrica intrínseca, denotada por $d_{G_{\mathcal{P}}}$, como subespaço de $S_{\mathcal{P}}$; também em $G_{\mathcal{P}}$ será considerada a medida $m_{G_{\mathcal{P}}}$ definida por $m_{G_{\mathcal{P}}}(D):=m_{\partial P}\left(\pi_{\mathcal{P}}^{-1}(D)\right)$, para todo Boreliano $D \subset G_{\mathcal{P}}$, sendo $m_{\partial P}$ a medida de Lebesgue 1-dimensional em $\partial P$.

Definição 2.2.3. Seja $(P, \mathcal{P})$ uma dobradura de papel e $S_{\mathcal{P}}$ o espaço de papel associado. Dado $k \in \mathbb{N} \cup\{\infty\}$, um ponto $x \in G_{\mathcal{P}}$ é um vértice de valência $k$ ou $k$-vértice, se $\# \pi_{\mathcal{P}}^{-1}(x)=k \neq 2$, ou se $\# \pi_{\mathcal{P}}^{-1}(x)=2$ e pelo menos uma das preimagem de $x$ é um vértice do multipolígono $P$.

Os pontos de um espaço de papel dividen-se em tres tipos:

- Pontos irregulares: Vértices de valência $\infty$ ou pontos de acumulação de vértices. Denote-se

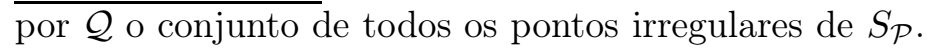

- Pontos cônicos: Vértices de valência $k$ não irregulares. Se a valência de um ponto cônico é $k$, chama-se ao ponto de $k$-cônico. Denote-se por $\mathcal{V}$ o conjuntos de pontos cônicos de $S_{\mathcal{P}}$. 
- Pontos planares: Os demais pontos de $S_{\mathcal{P}}$, é dizer os pontos em $S_{\mathcal{P}} \backslash(\mathcal{V} \cup \mathcal{Q})$.

Observação 2.2.4. Se $\left\langle\alpha, \alpha^{\prime}\right\rangle \in \mathcal{P}$ e $x \in \stackrel{\circ}{\alpha}$, é claro que $\pi_{\mathcal{P}}(x) \in S_{\mathcal{P}}$ é um ponto planar, pois $\pi_{\mathcal{P}}^{-1}\left(\pi_{\mathcal{P}}(x)\right)$ só tem dois ponto, nenhum deles vêrtice de $P$ (esse é o motivo de diferenciar na definição acima os 2 -vêrtices dos $k$-vêrtices com $k \neq 2$ ). Por outro lado as extremidades de segmentos pareados não necessariamente são projetados em pontos cônicos ou irregulares: Se $\left\langle\alpha, \alpha^{\prime}\right\rangle \in \mathcal{P}$, ambos segmentos podem-se dividir em dois subsegmentos $\alpha_{1} \cup \alpha_{2}, \alpha_{1}^{\prime} \cup \alpha_{2}^{\prime}$ com um único ponto común respectivamente, de forma que $\alpha_{1}, \alpha_{1}^{\prime}$ e $\alpha_{2}, \alpha_{2}^{\prime}$ estén pareados, logo neste novo pareamento $\mathcal{P}^{\prime}$, onde obviamente $S_{\mathcal{P}}=S_{\mathcal{P}^{\prime}}$, a extremidade común de $\alpha_{1}$ e $\alpha_{2}$ é projetado num ponto planar do espaço de papel. No entanto, é possível sempre escolher a dobradura de papel $(P, \mathcal{P})$ de forma que as extremidades de qualquer pareamento de segmentos sejam projetadas em pontos não-planares do espaço de papel; chaman-se a tais dobraduras de papel de eficientes, ver [Ber12], seção 2.4. Nesta tese sempre será assumido sem perda de generalidade que uma dobradura de papel $(P, \mathcal{P})$ é eficiente.

Proposição 2.2.5 ([dCH12]). Seja $(P, \mathcal{P})$ uma dobradura de papel e $G_{\mathcal{P}}$ a cicatriz do espaço de papel associado $S_{\mathcal{P}}$, então as seguintes propriedades são satisfeitas:

i) $O$ conjunto de pontos planares em $G_{\mathcal{P}}$ é aberto e denso em $G_{\mathcal{P}}$, enquanto que o conjunto $\mathcal{V} \cup \mathcal{Q}$ é fechado e de interior vazio em $G_{\mathcal{P}}$, com $m_{G_{\mathcal{P}}}$-medida nula. Em particular, o conjunto de pontos planares em $S_{\mathcal{P}}$ é aberto e denso.

ii) A métrica intrínseca $d_{G_{\mathcal{P}}}$ sobre $G_{\mathcal{P}}$ coincide com a métrica do quociente métrico $\partial P /\left(d_{\partial P}\right)^{\mathcal{P}}$.

iii) $G_{\mathcal{P}}$ tem dimensão de Hausdorff 1.

iv) Todo arco $\gamma$ em $G_{\mathcal{P}}$ é retificável, e $|\gamma|_{G_{\mathcal{P}}}=\frac{1}{2} m_{G_{\mathcal{P}}}(\gamma)$.

Definição 2.2.6. Considere-se um espaço de papel $S_{\mathcal{P}}$ associado à dobradura de papel $(P, \mathcal{P})$. O angulo de um ponto $x \in S_{\mathcal{P}} \backslash \mathcal{Q}$, denotado por $\xi_{\mathcal{P}}(x)$, define-se da seguinte forma: se $\pi_{\mathcal{P}}^{-1}(x)=$ $\left\{x_{1}, x_{2}, \ldots, x_{k}\right\}, k \geq 1$, então $\xi_{\mathcal{P}}(x):=\xi_{P}\left(x_{1}\right)+\xi_{P}\left(x_{2}\right)+\ldots+\xi_{P}\left(x_{k}\right)$, onde $\xi_{P}\left(x_{n}\right)$ denota o ângulo interno de $P$ no ponto $x_{n}$. A curvatura num ponto $x \in S_{\mathcal{P}} \backslash \mathcal{Q}$ define-se como $\kappa_{\mathcal{P}}(x):=2 \pi-\xi_{\mathcal{P}}(x)$.

Todo ponto planar num espaço de papel tem curvatura igual à cero. Por outro lado a curvatura num ponto 1-cônico qualquer é sempre un número positivo. Denote-se por $\mathcal{V}_{+}$o subespaço de $S_{\mathcal{P}} \backslash \mathcal{Q}$ dos pontos com curvatura positiva. Observe-se que o conjunto $\mathcal{V}_{+}$, além de conter todos os pontos 1-cônicos, podería conter alguns pontos $k$-cônicos com $k \geq 2$ mas, nesse caso, seriam somente um número finito de pontos: Se $x \in S_{\mathcal{P}}$ é $k$-cônico com $k \neq 1$ e $\kappa_{\mathcal{P}}(x)>0$, então alguma $\pi_{\mathcal{P}}$-preimagem de $x$ deve ser um vértice do polígono $P$.

Mostran-se a continuação alguns exemplos de espaços de papel para ilustrar mais claramente os conceitos antes definidos.

Exemplo 2.2.7. Considere-se $P$ um polígono regular de $n$-lados, e divida o $i$-ésimo lado em dois subsegmentos $\alpha_{i}$ e $\alpha_{i}^{\prime}, i=1,2, \ldots, n$, de igual comprimento. Considere-se $\mathcal{P}$ constituida por os $n$ pareamentos $\left\langle\alpha_{i}, \alpha_{i}^{\prime}\right\rangle$, ver figura 2.6. Observe-se que cada lado do polígono é dobrado pela metade, logo a identificação dos subsegmentos $\alpha_{i}$ e $\alpha_{i}^{\prime}$ é realizada por um isometría que reverte orientação com relação à $\partial P$. O espaço de papel associado $S_{\mathcal{P}}$ tem $n$ pontos 1-cônicos (também chamados de pontos de dobras) $p_{1}, p_{2}, \ldots, p_{n}$, cada um dos quais tem ângulo $\xi_{\mathcal{P}}\left(p_{i}\right)=\pi$, logo a curvatura é $\kappa_{\mathcal{P}}\left(p_{i}\right)=\pi$. Em $S_{\mathcal{P}}$ há um único ponto $n$-cônico $v$ cujas $\pi_{\mathcal{P}}$-preimagems são os vértices de $P$, com ângulo $\xi_{p}(v)=\pi(n-2)$. Observe-se que $\kappa_{\mathcal{P}}(v)<0$ se, e somente se, $n \geq 5$. 

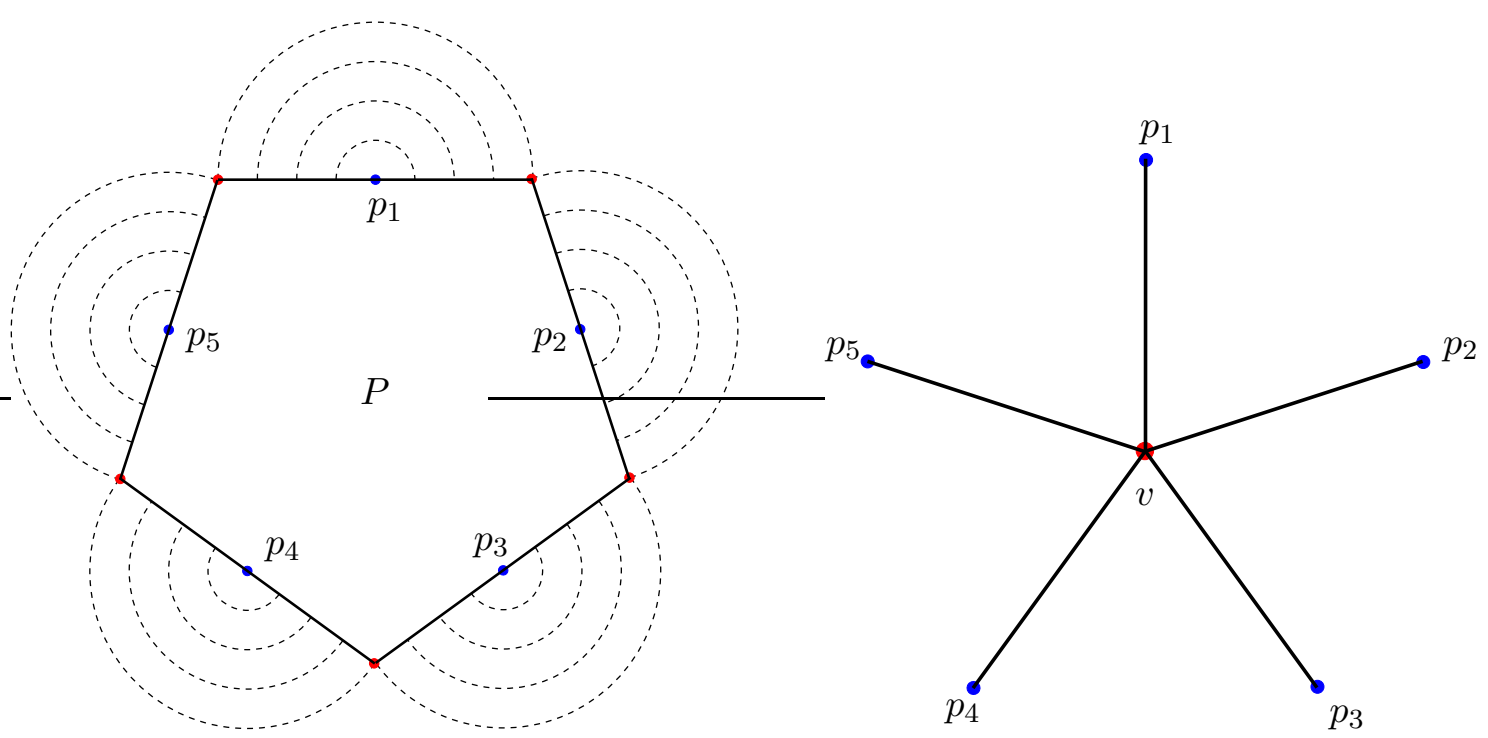

Figura 2.6: Espaço de papel do exemplo 2.2.7 para $n=5$ e sua cicatriz.

Exemplo 2.2.8. Considere-se novamente o quadrado unitario $P$ como na ferradura justa e a transformação $\hat{f}: P \longrightarrow P$ cuja ação é mostrada na figura 2.7. $\hat{f}$ de maneira similar a transformação da seção anterior não é bijetora nem continua. Porém identificando adequadamente subsegmentos de $\partial P$ como no caso da ferradura justa construye-se um espaço de papel $S=P / d^{\mathcal{P}}$ cujo pareamento é mostrado na figura 2.8 à esquerda, de forma que agora $\hat{f}$ projeta-se num homeomorfismo $f: S \longrightarrow S$ o qual será pseudo-Anosov generalizado. Este têm infinitos pontos 1-cônicos e 4-cônicos acumulandose em dois pontos $q^{1}$ e $q^{2}$, ambos de valência 1: o primeiro tem uma única preimagem no lado inferior do quadrado $P$ e o segundo tem uma única preimagem no lado esquerdo do quadrado $P$. Além dos pontos de valência 1 e $4, S_{\mathcal{P}}$ tem um único ponto 6-cônico, o qual tem ângulo $4 \pi$ (cuatro de suas seis preimagems são os vértices do $P$ ), logo de curvatura $-2 \pi$.

\section{$P$}

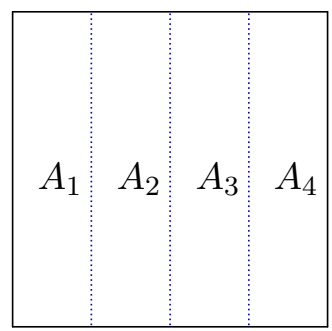

$P$

\begin{tabular}{|c|}
\hline${ }^{7} V$ \\
$A_{1}$ \\
${ }^{\nabla} V$ \\
\hline$A_{3}$ \\
\hline
\end{tabular}

Figura 2.7

Exemplo 2.2.9. O seguinte é um exemplo clásico de espaço quociente: O toro como quociente de um quadrado como mostra a figura 2.9 , na esquerda. O pareamento $\mathcal{P}$ consiste em duas translações: $z \mapsto z+i$ que identifica os lados horizontais, e $z \mapsto z+1$ que identifica os lados verticais, ambas isometrías revertem a orientação nos correspondentes segmentos pareados (Aínda as isometrías 

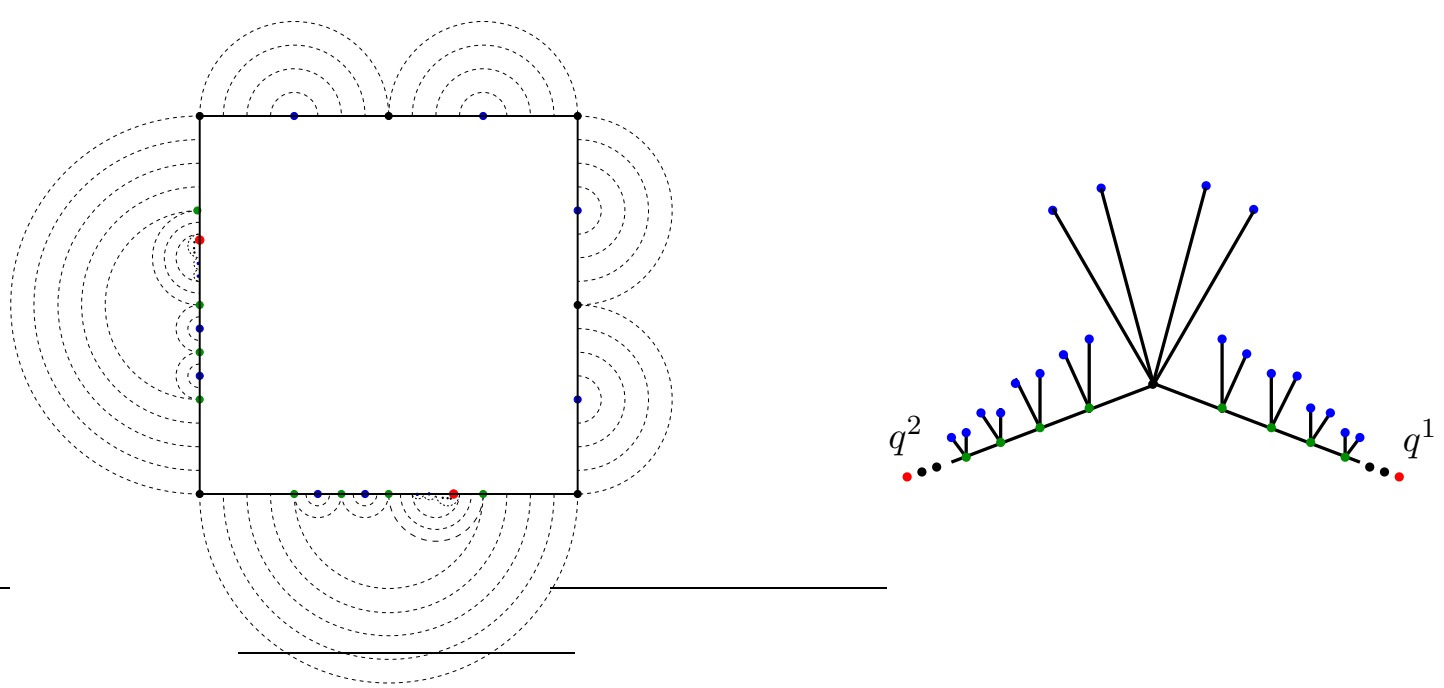

Figura 2.8: Espaço de papel do exemplo 2.2.8 e sua cicatriz. Os pontos azuis e os pontos verdes correspondem com pontos 1-cônicos e 4-cônicos respectivamente

preservam orientação vistas como transformações no plano). O espaço de papel associado tem um único ponto cônico de valência 4 e curvatura 0 correspondente à projeção dos vêrtices do quadrado.
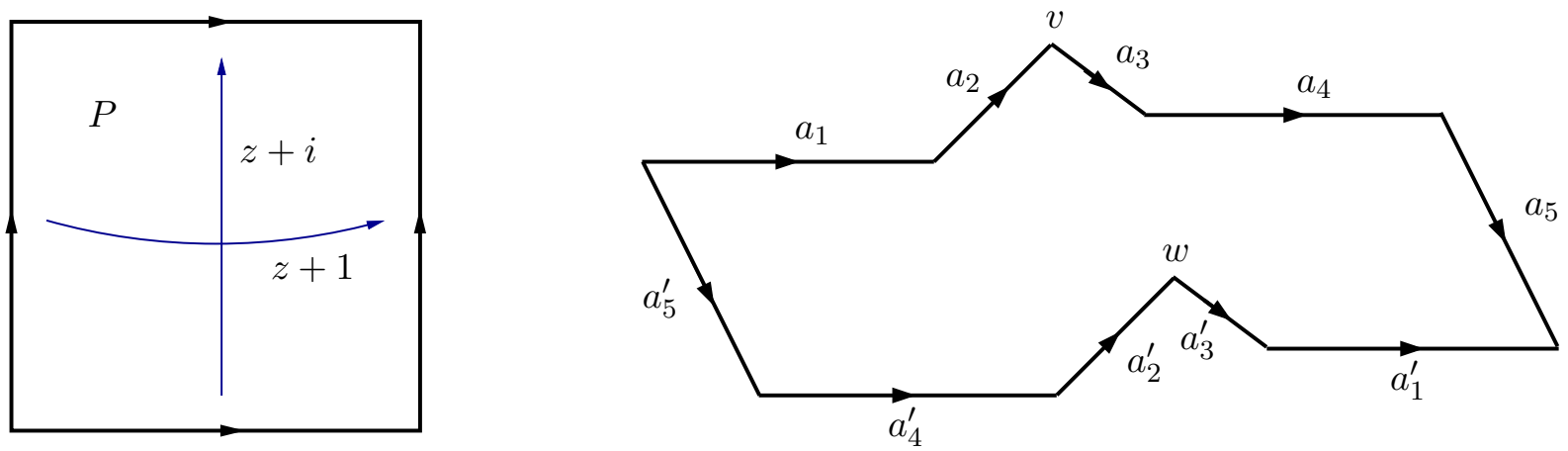

Figura 2.9: Espaço de papel que também são superfície de translação

De maneira mais geral, considere-se um polígono $P$ com as seguintes características: $O$ número de lados de $P$ é igual a $2 n, n \geq 2$, e se $a_{1}, a_{2}, \ldots a_{n}, a_{1}^{\prime}, a_{2}^{\prime}, \ldots a_{n}^{\prime}$ denotam os lados de $P$, existem vetores $t_{1}, t_{2}, t_{n}$ tais que para cada $1 \leq i \leq n, a_{i}^{\prime}=a_{i}+t_{i}$, ver figura 2.9 , na direita.

Tomando $\left.\mathcal{P}=\left\{\left\langle a_{i}, a_{i}^{\prime}\right\rangle / 1 \leq i \leq n\right\rangle\right\}$, o espaço de papel associado $S_{\mathcal{P}}$ é homeomorfo a uma superfície de gênero $g=\frac{n+\# \mathcal{V}-1}{2}$, onde o conjunto de pontos cônicos $\mathcal{V}$ de $S_{\mathcal{P}}$ é a $\pi_{\mathcal{P}}$-projeção dos vértices do polígono $P$. As isometrías que identificam $a_{i}$ com $a_{i}^{\prime}$ são definidas pelas translações $z \mapsto z+t_{i}$. Espaços de papel definidos deste jeito correspondem a uma categoría muito interesantes de espaços chamados de superfícies de translação, ver [Zor06]. No caso da figura $2.9, S_{\mathcal{P}}$ tem um ponto 2-cônico correspondente a $\pi_{\mathcal{P}}$-projeção do vértice común nos lados $a_{2}$ e $a_{3}, a_{2}^{\prime}$ e $a_{3}^{\prime}$; os demais vértices projetan-se num ponto 8-cônico, $\operatorname{logo} S_{\mathcal{P}}$ tem gênero $g=3$.

Uma superfície de translação mais extrema é a conhecida como a superfície de Chamanara, ver [Cha04], cujo pareamento é mostrado na figura 2.10. Fixado $\lambda \in(0,1)$, considere-se um quadrado $P$ de lado igual a $\sum_{j \geq 1} \lambda^{j}$, e divida os lados em sub-segmentos $a_{j}, b_{j}, a_{j}^{\prime}, b_{j}^{\prime}, j \geq 1$, como mostra a figura, onde $\left|a_{j}\right|=\lambda^{j}=\left|a_{j}^{\prime}\right|,\left|b_{j}\right|=\lambda^{j}=\left|b_{j}^{\prime}\right|$. 


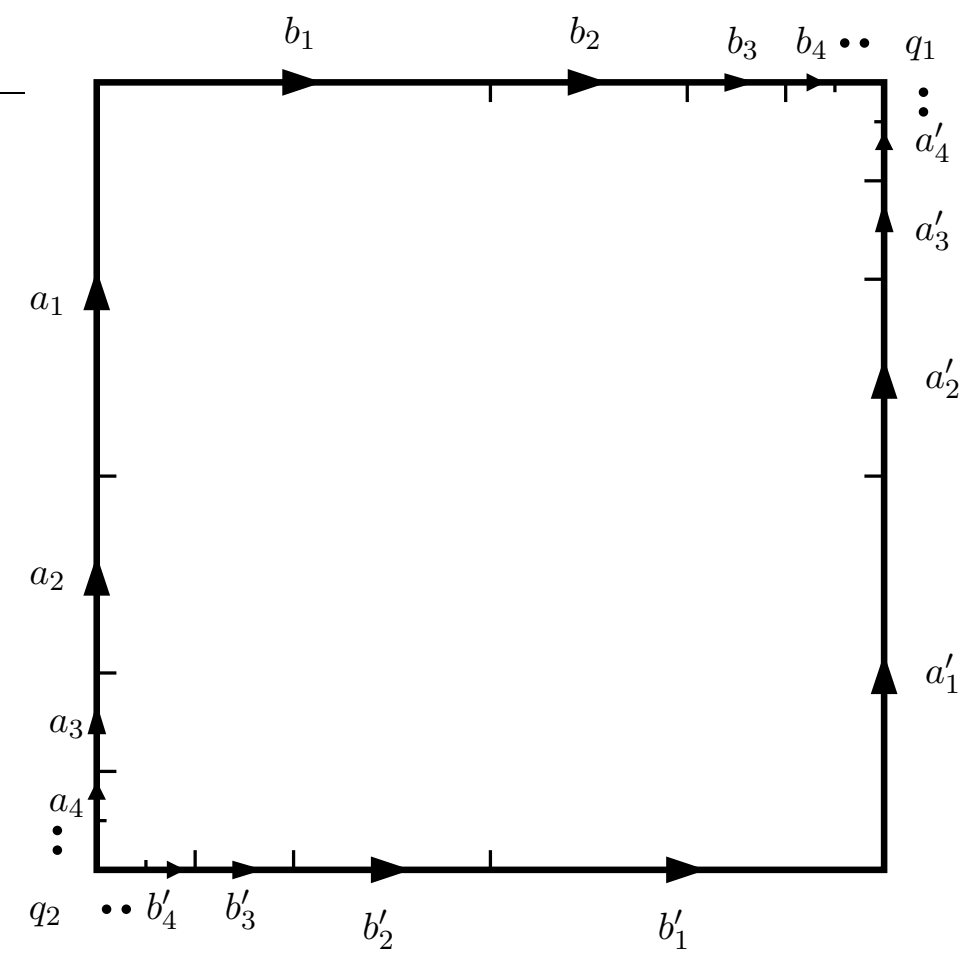

Figura 2.10: Superfície de Chamanara. Observe-se que $d^{\mathcal{P}}\left(q_{1}, q_{2}\right)=0$.

Para cada $j \geq 1$, o segmento $a_{j}$ é identificado com o segmento $a_{j}^{\prime}$ vía uma translação, e o segmento $b_{j}$ é identificado com $b_{j}^{\prime}$ também por uma translação. As extremidades de todos os segmentos, assim como os vértices superior-direito e inferior-esquerdo, projectam-se num único ponto irregular (pois tem valência $\infty$ ) o qual é o único ponto não-planar do espaço de papel. Observe-se que a cicatriz do espaço de papel consiste numa família infinita de círculos com um ponto común, o ponto irregular. O espaço de papel menos sua irregularidade é uma superfície de gênero infinito chamada freqüentemente de Monstro do lago Ness, ver [CGL06], [Ran16].

Exemplo 2.2.10. O seguinte exemplo é um espaço de papel $S_{\mathcal{P}}$ associado à dobradura $(P, \mathcal{P})$, onde $P$ é a união de cuatro retângulos $P=P_{1} \sqcup P_{2} \sqcup P_{3} \sqcup P_{4}$ do mesmo tamanho, ver figura 2.11. No disenho observa-se que $S_{\mathcal{P}}$ têm infinitos pontos 1-cônicos, todos de ângulo $\pi$, acumulando-se em dois pontos irregulares $q^{1}$ e $q^{2}$, os quais também são pontos de valência $\infty$.

Suponha que $\left\langle\alpha_{1}, \alpha_{1}^{\prime}\right\rangle$ é o pareamento em $\mathcal{P}$ que conecta $P_{1}$ e $P_{2}$, onde $\alpha_{1} \subset \partial P_{1}$ e $\alpha_{1}^{\prime} \subset$ $\partial P_{2}$. Igualmente sejam $\left\langle\alpha_{2}, \alpha_{2}^{\prime}\right\rangle,\left\langle\alpha_{3}, \alpha_{3}^{\prime}\right\rangle$ os pareamentos que conectam $P_{2}$ com $P_{3}$ e $P_{3}$ com $P_{4}$ respectivamente. Ao fazer as três identificações acima somente, é obtido um novo polígono $P^{\prime}$ como mostra a figura 2.12; observe-se que no interior do polígono $P^{\prime}$ estão contido três $\operatorname{arcos} \delta_{i}$ obtidos do colagem dos pareamentos $\left\langle\alpha_{i}, \alpha_{i}^{\prime}\right\rangle, i=1,2,3$. se $\mathcal{P}^{\prime}$ consiste nos pareamentos restantes de $\mathcal{P}$, claramente $S_{\mathcal{P}^{\prime}}=S_{\mathcal{P}}$. A figura 2.13 mostra a cicatriz do $S_{\mathcal{P}}$

Em [dCH12], é dado uma condição necessaria e suficiente para que um espaço de papel $S_{\mathcal{P}}$ associado à dobradura $(P, \mathcal{P})$ seja homeomorfo à uma superfície de gênero finito. Como nesta tese interesa principalmente o caso dos espaços de papel homeomorfos à esferas, não será desenvolvida 


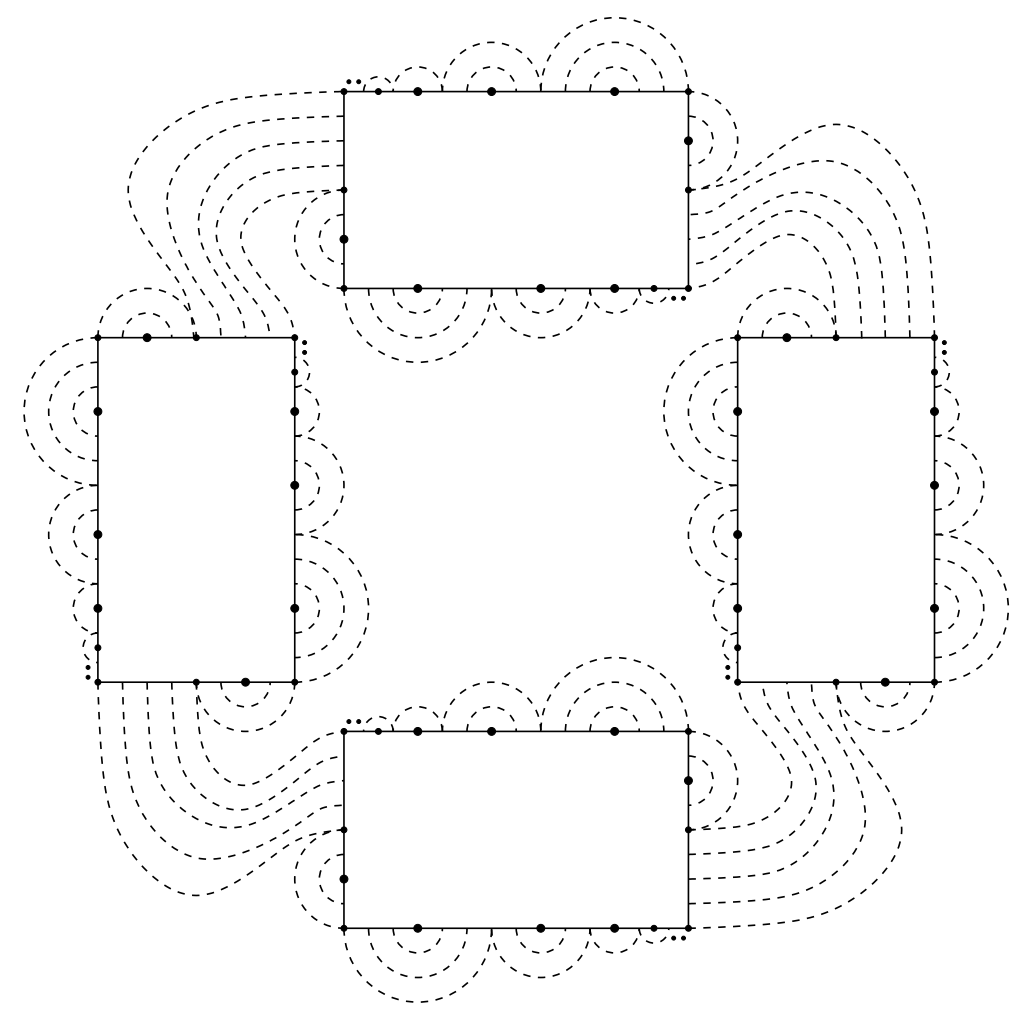

Figura 2.11: Espaço de papel do exemplo 2.2.10 obtido como quociente de cuatro rectángulos

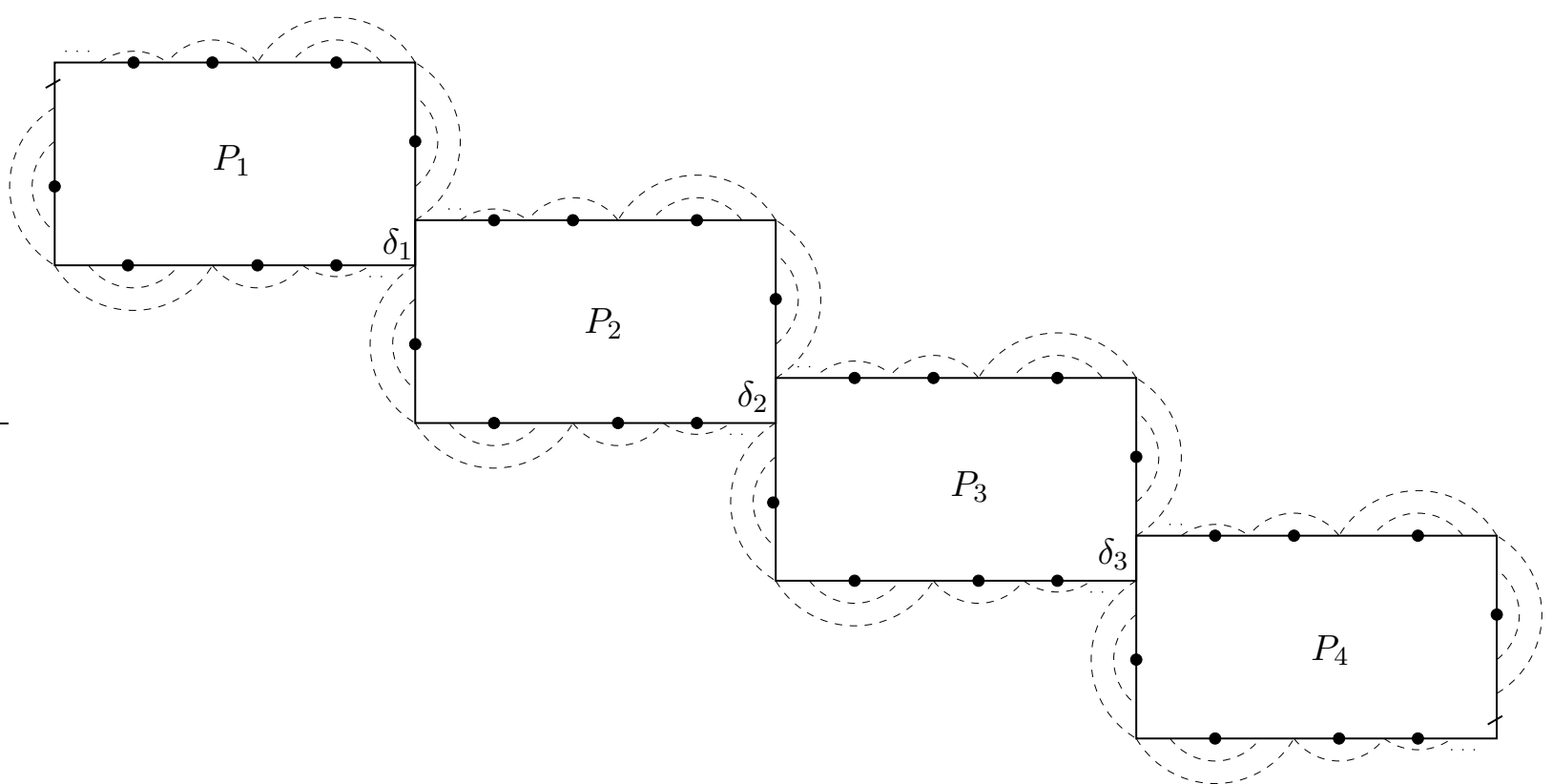

Figura 2.12: Espaço de papel associado à dobradura $\left(P^{\prime}, \mathcal{P}^{\prime}\right)$ do exemplo 2.2.10

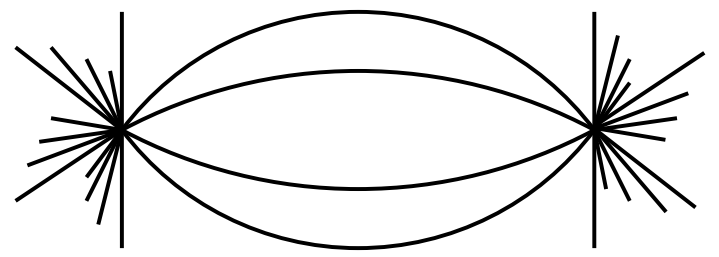

Figura 2.13: Cicatriz do espaço de papel do exemplo 2.2.10 
esta caracterização geral, mas sim será caracterizado os espaços de papel de gênero 0 na seguinte seção para fazer o texto o mais auto-contido possível. Por enquanto os seguintes dois resultados são conseqüências direitas da caracterização feita em [dCH12].

Proposição 2.2.11 ([dCH12]). Se o espaço de papel $S_{\mathcal{P}}$ é homeomorfo à uma superfície de gênero finito, então sua cicatriz $G_{\mathcal{P}}$ é um dendrito local.

Proposição 2.2.12 ([dCH12]). Se o espaço de papel $S_{\mathcal{P}}$ não é homeomorfo à uma superfície de gênero finito, então $S_{\mathcal{P}}$ contém uma superfície de gênero infinito.

Por outro lado é fácil ver que em qualquer espaço de papel $S_{\mathcal{P}}$, se $x \in S_{\mathcal{P}}$ é um ponto planar, existe $r>0$ tal que a bola $B_{S_{\mathcal{P}}}(x, r)$ é isométrica à bola $B_{\mathbb{C}}(0, r)$. No capítulo 2 de [Ber12] provase com detalhe que em cada ponto cônico $x \in S_{\mathcal{P}}$ de ângulo $\xi_{\mathcal{P}}(x)$, existe $r>0$ suficientemente pequenho tal que a bola $B_{S_{\mathcal{P}}}(x, r)$ é isométrica à bola de raio $r$ e centro [0] no cone métrico Cone $\left(\frac{\xi_{\mathcal{P}}(p)}{2 \pi}\right)$. Em conseqüência, em todo espaço de papel $S_{\mathcal{P}}$, o subespaço $S_{\mathcal{P}} \backslash \mathcal{Q}$ está equipado de uma estrutura de superfície cônico-plana, com o qual possui uma estrutura complexa natural associada á tal estrutura cônico-plana. A pergunta agora é se tal estrutura complexa pode ser extendida ao conjunto de pontos irregulares fazendo todo o espaço $S_{\mathcal{P}}$ uma superfície de Riemann. No mesmo artigo [dCH12] (veja-se também [dCH13]) mostra-se um criterio que garante a extensão da estrutura complexa ao conjunto de pontos $\mathcal{Q}$. Considere-se o seguinte: Dado $q \in G_{\mathcal{P}}$ e $r>0$ suficientemente pequenho, as funções $m_{q}(r)$ e $n_{q}(r)$ são definidas por,

$$
\begin{gathered}
m_{q}(r):=m_{G_{\mathcal{P}}}\left(B_{G_{\mathcal{P}}}(q, r)\right) \\
n_{q}(r):=\# \mathbb{S}_{G_{\mathcal{P}}}(q, r)
\end{gathered}
$$

Teorema 2.2.13 (ver [dCH12], Teorema 59). Seja $S_{\mathcal{P}}$ um espaço de papel de gênero finito associado à dobradura $(P, \mathcal{P})$. Se para todo $q \in \mathcal{Q}$,

$$
\int_{0} \frac{d r}{m_{q}(r)+r \cdot n_{q}(r)}=\infty
$$

então a estrutura complexa de $S_{\mathcal{P}} \backslash \mathcal{Q}$ extende-se de maneira única para toda a superfície de papel.

É importante resaltar aqui que aínda fica em aberto conhecer se sempre é possível extender a estrutura complexa aos pontos $\mathcal{Q}$ num espaço de papel arbitrario de gênero finito. Na maioria dos exemplos interesantes em algum sentido a resposta é afirmativa, mas é fácil conseguir outros exemplos com irregularidades isoladas onde não se sabe se tal extensão acontece.

\subsection{Esferas de papel}

O objetivo principal desta seção é mostrar uma condição necessaria e suficiente para que o espaço de papel associado a uma dobradura $(P, \mathcal{P})$ seja uma esfera topológica. Como já foi mencionado na seção anterior, tal caracterização foi feita em [dCH12] (Ver Teorema 42) no caso onde $P$ é um único polígono; porém a prova vale também para o caso onde $P$ é um multipolígono e por isso será reproduzida a prova desse caso nesta seção. Também será demostrado a proposição 2.2.11 para o 
caso particular dos espaços de papel de gênero 0, os quais serão chamados simplemente de esferas de papel.

Considere-se uma dobradura de papel $(P, \mathcal{P})$ onde o multipolígono $P$ é a união dos polígonos $P_{1} \cup P_{2} \cup \ldots \cup P_{k}$. Seja $\Upsilon_{\mathcal{P}}$ o grafo simple cujos vértices são os polígonos $P_{i}$, e existe uma aresta conectando o vértice $P_{i}$ com o vértice $P_{j}, i \neq j$, se e somente se, existe um pareamento $\left\langle\alpha, \alpha^{\prime}\right\rangle \in \mathcal{P}$ tal que $\alpha \subset \partial P_{i}$ e $\alpha^{\prime} \subset \partial P_{j}$. Claramente o espaço de papel associado $S_{\mathcal{P}}$ é conexo se, e somente se, o grafo $\Upsilon_{\mathcal{P}}$ é conexo; nesta tese também será sempre considerada esta hipótese. Seja $T$ um árvore de extensão do grafo $\Upsilon_{\mathcal{P}}$, escolha-se um pareamento $\left\langle\alpha_{l}, \alpha_{l}^{\prime}\right\rangle$ associado a cada aresta de $T$ e cole todos os polígonos $P_{i}$ vía as identificações escolhidas. O espaço resultante é um disco topológico $P^{\prime}$ e os restantes pareamentos de $\mathcal{P}$ induzem um pareamento $\mathcal{P}^{\prime}$ de $\operatorname{arcos}$ de $\partial P^{\prime}$ interiormente disjunta e total (Ver a figura 2.12 no exemplo 2.2.10). Ainda o disco $P^{\prime}$ não é um polígono no sentido descrito ao começo da seção 2.2, a estrutura poligonal é irrelevante para a estrutura topológica do quociente $P^{\prime} / d^{\mathcal{P}^{\prime}}$. Por outro lado, sendo o quociente métrico $P^{\prime} / d^{\mathcal{P}^{\prime}}$ igual ao quociente métrico $P / d^{\mathcal{P}}$, também não é relevante a escolha do árvore de extensão $T$ do grafo $\Upsilon_{\mathcal{P}}$ nem as escolhas dos pareamentos associados as arestas do árvore $T$. Para garantir que o espaço de papel $S_{\mathcal{P}}$ seja uma homeomorfo a uma esfera precisa-se dar condições para que o quociente $P^{\prime} / d^{\mathcal{P}^{\prime}}$ seja uma esfera. Por simplicidade, as seguintes definições e proposições serám feitas para o caso que $P$ seja um único polígono, mas valerão também para o caso do disco $P^{\prime}$ obtido ao colar parcialmente os polígonos de um multipolígono qualquer como foi explicado anteriormente.

Definição 2.3.1. Seja $\gamma$ um arco ou laço poligonal. Dois pares de pontos não necessariamente distintos $\left\{x, x^{\prime}\right\},\left\{y, y^{\prime}\right\} \subset \gamma$ são desvinculados, se um dos pares está contido no fecho de uma componente conexa do complemento do outro; caso contrario, eles são vinculados. Uma relação simétrica e reflexiva $R$ em $\gamma$ é desvinculada, se qualquer par de pares $\left\{x, x^{\prime}\right\},\left\{y, y^{\prime}\right\} \subset \gamma$, com $x R x^{\prime}$, $y R y^{\prime}$ e $x$ e $x^{\prime}$ não identificado por $R$ nem a $y$ nem a $y^{\prime}$, são desvinculados.

Definição 2.3.2. Seja $(P, \mathcal{P})$ uma dobradura de papel e $\gamma \subset \partial P$ um arco ou laço (em cujo caso sería $\gamma=\partial P$ ). dize-se que $\gamma$ é $\mathcal{P}$-simple se,

i) A restrição de $\mathcal{P}$ à $\gamma$ é desvinculada.

ii) Se $\left\langle\alpha, \alpha^{\prime}\right\rangle$ é um pareamento de $\mathcal{P}$ e $\alpha \cap$ int $\gamma \neq \emptyset$ ou $\alpha^{\prime} \cap$ int $\gamma \neq \emptyset$, então $\alpha \cup \alpha^{\prime} \subset \gamma$.

Lema 2.3.3 ([dCH12]). Se $\gamma \subset \partial P$ é $\mathcal{P}$-simple, suas extremidades são $\sim_{\mathcal{P}}$-equivalentes.

Demonstração. Sejam $a$ e $b$ as extremidades de $\gamma$, logo basta provar que para todo $\varepsilon>0, d^{\mathcal{P}}(a, b)<$ $\varepsilon$. Por conveniencia, considere-se os pontos de $\gamma$ ordenados de esquerda a direita, sendo $a$ a extremidade esquerda e $b$ a direita. Pela totalidade de $\mathcal{P}$, existe uma familía finita de pareamentos $\left\{\left\langle\alpha_{j}, \alpha_{j}^{\prime}\right\rangle\right\}_{j=1}^{N}$ contida em $\gamma$ tal que $\cup_{j=1}^{N}\left(\alpha_{j} \cup \alpha_{j}^{\prime}\right)$ cobre $\gamma$ a menos de comprimento menor que $\varepsilon$. Suponha que para todo $j$ o segmento $\alpha_{j}$ está a esquerda do segmento $\alpha_{j}^{\prime}$.

Seja $p_{0}=a$ e construya-se um $\mathcal{P}$-itinerário de $a$ até $b$ inductivamente da seguinte manera: Para cada $i \geq 1$, seja $q_{i}$ o ponto de $\left[p_{i}, b\right]$ más próximo de $p_{i}$ que seja uma extremidade esquerda de algum $\alpha_{j(i)}$; Seja $p_{i+1}$ a extremidade direita de $\alpha_{j(i)}^{\prime}$, logo $\left(q_{i}, p_{i+1}\right) \in \mathcal{P}$. Como a familía de pareamentos é finita, existe $k \geq 1$ tal que $\left[p_{k}, b\right]$ é disjunto de todos os $\alpha_{j}$; tome $q_{k}=b$. Por construção $p_{0} \leq$ $q_{0}<p_{1} \leq q_{1}<\ldots<p_{k} \leq q_{k}$. Os intervalos $\left(p_{i}, q_{i}\right)$ são disjuntos de cada $\alpha_{j}$ pela escolha de $q_{i}$. Por outro lado, os intervalos $\left(p_{i}, q_{i}\right)$ são também disjuntos de cada $\alpha_{j}^{\prime}$ pois, se $\left(p_{i}, q_{i}\right)$ intersecta algum $\alpha_{j}^{\prime}$, então $\alpha_{j}$ deve estar contida em $\left(q_{s}, p_{s+1}\right)$ para algum $s<j$, logo $\alpha_{j(s)}<\alpha_{j}<\alpha_{j(s)}^{\prime}<\alpha_{j}^{\prime}$ seriam vinculados, contrariando a simplicidade de $\gamma$. Assim $\cup_{i=0}^{k}\left(p_{i}, q_{i}\right) \subset \gamma \backslash \cup_{j=1}^{N}\left(\alpha_{j} \cup \alpha_{j}^{\prime}\right)$, pelo qual,

$$
d^{\mathcal{P}}(a, b) \leq L^{\mathcal{P}}\left(\left(p_{i}, q_{i}\right)_{i=0}^{k}\right)=\sum_{i=0}^{k} d\left(p_{i}, q_{i}\right)<\varepsilon
$$


Suponha que $\left\langle\alpha, \alpha^{\prime}\right\rangle$ é um pareamento de $\mathcal{P}, \beta$ é um sub-segmento de $\alpha$ e $\beta^{\prime}$ é o correspondente sub-segmento de $\alpha^{\prime}$. dize-se neste caso que $\left\langle\beta, \beta^{\prime}\right\rangle$ é um sub-pareamento de $\left\langle\alpha, \alpha^{\prime}\right\rangle$.

Lema 2.3.4 ([dCH12]). Seja $\gamma$ um arco $\mathcal{P}$-simple em $\partial P,\left\langle\alpha, \alpha^{\prime}\right\rangle$ um sub-pareamento de algum

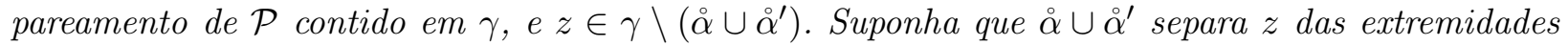
de $\gamma$. então o comprimento de $\left|\left\langle\alpha, \alpha^{\prime}\right\rangle\right|$ é uma cota inferior de $d_{\partial P}^{\mathcal{P}}(z, w)$, para todo $w \in \partial P$ que não

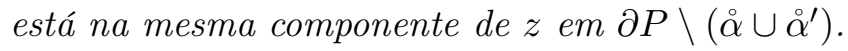

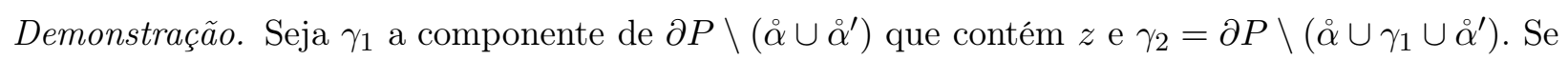
$x \in \gamma_{1}$ e $y \in \partial P$ é um ponto tal que $x \mathcal{P} y$, existe um sub-pareamento $\left\langle\beta, \beta^{\prime}\right\rangle$ tal que $x \in \beta, y \in \beta^{\prime}$, e $\stackrel{\circ}{\beta} \cup \dot{\beta}^{\prime}$ é disjunto de $\stackrel{\alpha}{\alpha} \stackrel{\circ}{\alpha}^{\prime}$. Claramente $\beta \subset \gamma_{1}, \operatorname{logo} \beta^{\prime} \subset \gamma$. Como a relação $\mathcal{P}$ é desvinculada em $\gamma$, tem-se que $\beta^{\prime} \subset \gamma_{1}$, logo $y \in \gamma_{1}$. Isto prova que $\gamma_{1}$ é $\mathcal{P}$-saturado. Um argumento similar mostra que $\gamma_{2}$ também é $\mathcal{P}$-saturado.

Para demostrar que $d_{\partial P}^{\mathcal{P}}(z, w) \geq\left|\left\langle\alpha, \alpha^{\prime}\right\rangle\right|$, para todo $w \in \gamma_{2}$, basta provar que o $d_{\partial P^{-c u s t o}}$ de qualquer $\mathcal{P}$-itinerário $\left\{\left(p_{i}, q_{i}\right)\right\}_{i=0}^{N}$ de $z$ à $w$ é limitado inferiormente por $\left|\left\langle\alpha, \alpha^{\prime}\right\rangle\right|$. A saturação de $\gamma_{1}$ e $\gamma_{2}$ implicam que existem $r \leq s$ tal que $p_{r}$ é o último ponto no $\mathcal{P}$-itinerário em $\gamma_{1}$ e $q_{s}$ é o primeiro ponto no $\mathcal{P}$-itinerário em $\gamma_{2}$.

Se $r=s, L^{\mathcal{P}}\left(\left(p_{i}, q_{i}\right)_{i=0}^{N}\right) \geq d_{\partial P}^{\mathcal{P}}\left(p_{r}, q_{s}\right) \geq\left|\left\langle\alpha, \alpha^{\prime}\right\rangle\right|$. Se $r<s$, os pontos $q_{r}, p_{r+1}, q_{r+1}, \ldots, p_{s}$ estão em $\alpha \cup \alpha^{\prime}$. Sejam $\zeta, \zeta^{\prime}:\left[0,\left|\left\langle\alpha, \alpha^{\prime}\right\rangle\right|\right] \longrightarrow \partial P$ parametrizações de $\alpha$ e $\alpha^{\prime}$ por comprimento de arco, e sejam $t_{i}, r \leq i<s$, parámetros tais que $\zeta\left(t_{i}\right)=q_{i}, \zeta\left(t_{i}\right)=p_{i+1}$, então

$$
L^{\mathcal{P}}\left(\left(p_{i}, q_{i}\right)_{i=0}^{N}\right) \geq \sum_{i=r}^{s} d_{\partial P}\left(p_{i}, q_{i}\right) \geq t_{r}+\sum_{i=r+1}^{s-1}\left|t_{i}-t_{i-1}\right|+||\left\langle\alpha, \alpha^{\prime}\right\rangle\left|-t_{s}\right| \geq\left|\left\langle\alpha, \alpha^{\prime}\right\rangle\right|
$$

Lema 2.3.5 ([dCH12]). Se $\gamma \subset \partial P$ é um arco $\mathcal{P}$-simple e $a, b$ denota suas extremidades, então a restrição de $\sim_{\mathcal{P}}$ em $\gamma$ é desvinculada e $\gamma \backslash[a]=\gamma \backslash[b]$ é $\sim_{\mathcal{P}}$-saturada, onde $[x]$ denota $a \sim_{\mathcal{P}}$-classe de equivalência de um ponto $x$.

Demonstração. Suponha-se que os pontos de $\gamma$ estão ordenados de esquerda à direita, sendo $a$ a extremidade esquerda. Para provar que $\sim_{\mathcal{P}}$ é desvinculado em $\gamma$, basta mostrar que, se existem pontos $x, x^{\prime}, y, y^{\prime} \in \gamma$ tais que $a \leq x<y<x^{\prime}<y^{\prime} \leq b, x \sim_{\mathcal{P}} x^{\prime}$ e $y \sim_{\mathcal{P}} y^{\prime}$, então $y \sim_{\mathcal{P}} x^{\prime}$ (Neste caso os cuatro pontos estão $\sim_{\mathcal{P}}$-relacionados); para provar isso basta mostrar, usando o lema 2.3.3, que o arco $\left[y, x^{\prime}\right]$ é simple. Como a relação $\mathcal{P}$ em $\gamma$ é desvincualda, ela também é desvinculada em $\left[y, x^{\prime}\right]$. Suponha por redução ao absurdo que $\left[y, x^{\prime}\right]$ não é simple, logo existe um sub-pareamento $\left|\left\langle\alpha, \alpha^{\prime}\right\rangle\right|$ de algum pareamento de $\mathcal{P}$ tal que $\alpha \subset\left(y, x^{\prime}\right)$ e $\alpha^{\prime} \subset \gamma \backslash\left[y, x^{\prime}\right]$. Se $\alpha^{\prime} \subset[a, y)$, pelo lema 2.3.4, $d^{\mathcal{P}}\left(y, y^{\prime}\right)>0$, logo $y \nsim_{\mathcal{P}} y^{\prime}$, e se $\alpha^{\prime} \subset\left(x^{\prime}, b\right]$, então pelo mesmo lema $d^{\mathcal{P}}\left(x, x^{\prime}\right)>0$, logo $x \nsim_{\mathcal{P}} x^{\prime}$. As duas opções leva a uma contradição, pelo qual $\left[y, x^{\prime}\right]$ é simple.

Para demostrar que $\gamma \backslash[a]$ é $\sim_{\mathcal{P}}$-saturado é suficiente provar que dado qualquer $x \in \dot{\gamma}, x \sim_{\mathcal{P}} a$ ou a $\sim_{\mathcal{P}}$-classe de equivalência de $x$ está contida em $\gamma$. Se existe um sub-pareamento em $\gamma$ que separa a $x$ de $a$ e $b$, pelo lema 2.3.4 tem-se que $d^{\mathcal{P}}(z, w)>0$ para todo $w \in \partial P \backslash \gamma$, $\log [x] \subset \gamma$. Se nemhum sub-pareamento separa a $x$ das extremidades de $\gamma$, então os arcos $[a, x]$ e $[x, b]$ são simples, $\log 0[x]=[a]=[b]$.

Definição 2.3.6. Seja $P=P_{1} \cup P_{2} \cup \ldots \cup P_{k}, k \geq 1$, um multipolígono e $\mathcal{P}$ uma coleção de pareamentos de $P$. A dobradura de papel $(P, \mathcal{P})$ é simple se para alguma escolha da dobradura $\left(P^{\prime}, \mathcal{P}^{\prime}\right)$ associada a um árvore de extensão de $\Upsilon_{\mathcal{P}}$, a relação $\mathcal{P}^{\prime}$ é desvinculada em $\partial P^{\prime}$. 
Observação 2.3.7. $\quad$ i) Para o caso $k=1$, tem-se trivialmente que $P=P^{\prime}$ e $\mathcal{P}=\mathcal{P}^{\prime}$, logo a dobradura $(P, \mathcal{P})$ é simple se $\mathcal{P}$ é desvinculada em $\partial P$.

ii) Em principio a definição acima parece depender da escolha da dobradura $\left(P^{\prime}, \mathcal{P}^{\prime}\right)$, mas como será mostrado no seguinte resultado (que é a caracterização das esferas de papel), se $\left(P^{\prime \prime}, \mathcal{P}^{\prime \prime}\right)$ é outra escolha de dobradura associada a otro árvore de extensão de $\Upsilon_{\mathcal{P}}$, a relação $\mathcal{P}^{\prime \prime}$ é desvinculada em $\partial P^{\prime \prime}$ se $\mathcal{P}^{\prime}$ é desvinculada em $\partial P^{\prime}$.

Teorema 2.3.8 ([dCH12]). O espaço de papel $S_{\mathcal{P}}$ associado a dobradura de papel $(P, \mathcal{P})$ é uma esfera topológica se, e somente se, a dobradura $(P, \mathcal{P})$ é simple.

Demonstração. Suponha que $(P, \mathcal{P})$ é simple e seja $\left(P^{\prime}, \mathcal{P}^{\prime}\right)$ uma dobradura associada a um árvore de extensão de $\Upsilon_{\mathcal{P}}$ tal que $\mathcal{P}^{\prime}$ é desvinculada em $\partial P^{\prime}$. Lembre-se que $\mathcal{P}^{\prime}$ é um disco topológico pelo qual existe um homeomorfismo que leva $\partial P^{\prime}$ no círculo unitario $\mathbb{S}^{1} \subset \mathbb{C}$, e leva o interior do disco $P^{\prime}$ no exterior do disco unitario $\mathbb{D} \subset \widehat{\mathbb{C}}$, pelo qual identifica-se o disco $P^{\prime}$ com $\mathbb{C} \backslash \mathbb{D}$. A ideia agora é construir uma decomposição $\mathcal{G}$ de $\widehat{\mathbb{C}}$ monótona e semi-continua superiormente de forma que $P^{\prime} / d^{\mathcal{P}^{\prime}} \cong \hat{\mathbb{C}} / \mathcal{G}$, logo pelo Teorema 1.1 .15 o espaço de papel $S_{\mathcal{P}}=P / \mathcal{P}=P^{\prime} / \mathcal{P}^{\prime}$ é homeomorfo a uma 2-esfera. É importante salientar que a identificação de $P^{\prime}$ com $\widehat{\mathbb{C}} \backslash \mathbb{D}$ é simplemente uma mudança de coordenadas conveniente e que a construção de $\mathcal{G}$ não usa a geometría hiperbólica de nenhuma maneira essencial.

Se $\left\langle\alpha, \alpha^{\prime}\right\rangle$ é um pareamento de $\mathcal{P}^{\prime}$, dois pontos pareados por tal pareamento que estão no interior de $\alpha$ e de $\alpha^{\prime}$ são chamados de pares de pontos interiores. Conecte-se cada par de pontos interiores em $\partial \mathcal{P}^{\prime}$ com a linha geodésica em $\mathbb{D}$ que tem tais pontos como extremidades. O fato de ser a relação $\mathcal{P}^{\prime}$ em $\partial P^{\prime}$ desvinculada garante que a coleção de geodésicas assim construidas sejam duas a duas disjuntas. Seja $\mathcal{L}$ a laminação geodésica obtida do fecho da união de todas as linhas geodésicas que conectam pares de pontos interiores de $\partial P^{\prime}$. Como a relação $\sim_{\mathcal{P}^{\prime}}$ é fechada, toda geodésica em $\mathcal{L}$

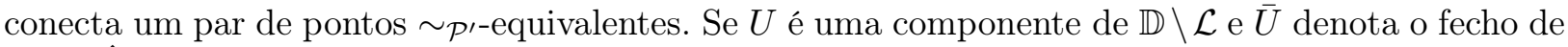
$U$ em $\hat{\mathbb{C}}$, então $\bar{U}$ é um disco fechado cuja fronteira é a união de algumas geodésicas em $\mathcal{L}$, incluindo suas extremidades em $\mathbb{S}^{1}$, conectando pontos $\sim_{\mathcal{P}^{\prime}}$-equivalentes que não são pares interiores.

Sejam $x, y \in \partial P^{\prime}$ dois pontos diferentes e que não estão em um par interior, e seja $[x, y] \subset \partial P^{\prime}$ um arco com extremidades $x$ e $y$. As seguintes condições são equivalentes:

i) $x \sim \sim_{\mathcal{P}^{\prime}} y$.

ii) $[x, y]$ é $\mathcal{P}^{\prime}$-simple.

iii) $x, y$ pertencem à $\bar{U}$, sendo $U$ uma componente de $\mathbb{D} \backslash \mathcal{L}$ ou, $x, y$ são as extremidades de uma geodésica em $\mathcal{L}$.

Para provar i) $\Leftrightarrow$ ii), suponha que $[x, y]$ não é $\mathcal{P}^{\prime}$-simple. $\mathcal{P}^{\prime}$ é desvinculado em $\partial P^{\prime}$ e como $x$ nem $y$ estão num par interior, deve existir um pareamento $\left\langle\alpha, \alpha^{\prime}\right\rangle$ em $\mathcal{P}^{\prime}$ tal que $\alpha \subset[x, y] \mathrm{e}$ $\alpha^{\prime} \cap(x, y)=\emptyset$. Isto implica que $x$ e $y$ pertencem a diferentes componentes de $\partial P^{\prime} \backslash\left(\stackrel{\circ}{\alpha} \cup \dot{\alpha}^{\prime}\right)$, $\operatorname{logo}$ pelo lema 2.3.4 e considerando a $\partial P^{6}$ como o arco $\mathcal{P}^{\prime}$-simple com a mesma extremidade $x$, tem-se que $x \nsim_{\mathcal{P}^{\prime}} y$. O recíproco é conseqüência do lema 2.3.3.

Para provar ii) $\Leftrightarrow$ iii), suponha que $[x, y]$ é $\mathcal{P}^{\prime}$-simple e que a linha geodésica com extremidades $x$ e $y$ não pertence à $\mathcal{L}$. Se existe uma geodésica em $\mathcal{L}$ com uma extremidade em $(x, y)$ e a outra fora de $[x, y]$ existirá uma geodésica com extremidade $a \in(x, y)$ e $b \in \partial P^{\prime} \backslash[x, y]$, sendo $a$ e $b$ um

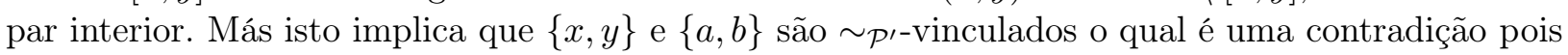
$\sim \mathcal{P}^{\prime}$ é desvinculado em $\partial P^{\prime}$. Assim, não existe uma geodésica com um extremo em $(x, y)$ e o outro em $\partial P^{\prime} \backslash[x, y]$, logo a geodésica que conecta $x$ e $y$ está contido numa componente de $\mathbb{D} \backslash \mathcal{L}$. Se $[x, y]$ não é $\mathcal{P}^{\prime}$-simple, existe um pareamento $\left\langle\alpha, \alpha^{\prime}\right\rangle$ em $\mathcal{P}^{\prime}$ tal que $\stackrel{\alpha}{\alpha} \subset(x, y)$ e ${ }^{\prime} \cap[x, y]=\emptyset$, $\operatorname{logo}$ qualquer geodésica que conecta pontos pareados em $\stackrel{\alpha}{\cup} \cup \stackrel{\circ}{\alpha}^{\prime}$ separa a $x$ de $y$ em $\overline{\mathbb{D}}$. 
Das equivalências mostradas acima conclui-se que dois pontos diferentes $x, y \in \partial P^{\prime}$ são $\sim_{\mathcal{P}^{\prime-}}$ relacionados se, e somente se, ou eles são extremidades de uma geodésica em $\mathcal{L}$ ou eles estão no mesmo fecho de uma componente de $\mathbb{D} \backslash \mathcal{L}$. Seja $\mathcal{G}$ a decomposição de $\hat{\mathbb{C}}$ que consiste em elementos dos seguintes tipos:

I) Fecho de uma componente de $\mathbb{D} \backslash \mathcal{L}$.

II) Geodésica em $\mathcal{L}$ incluindo suas extremidades, que não estão em elementos do tipo II).

III) Pontos que não estão em elementos do tipo I) e II).

Como $P^{\prime}$ é compacto, o quociente métrico $P^{\prime} / d^{\mathcal{P}^{\prime}}$ é homeomorfo ao quociente topológico $P^{\prime} / \sim_{\mathcal{P}^{\prime}}$ o qual, pelos argumentos feitos antes, coincide com o quociente topológico $\hat{\mathbb{C}} / \mathcal{G}$. Por outro lado, os elementos de $\mathcal{G}$ sendo discos fechados, arcos simples e pontos, são conexos e não separam a $\hat{\mathbb{C}}$. Logo para aplicar o Teorema de Moore somente falta provar que a relação de equivalência $R_{\mathcal{G}}$ é fechada.

Sejam $\left(x_{n}\right)_{n \geq 1}$ e $\left(y_{n}\right)_{n \geq 1}$ sequências em $\hat{\mathbb{C}}$ tais que $x_{n} \rightarrow x, y_{n} \rightarrow y$ e para cada $n \geq 1$ existe um elemento $g_{n} \in \mathcal{G}$ tal que $x_{n}, y_{n} \in g_{n}$. Procura-se provar que existe $g \in \mathcal{G}$ tal que $x, y \in g$. Podemos assumir sem perda de generalidade que todos os elementos $g_{n}$ são do mismo tipo.

Se os $g_{n}$ são todos do tipo III), então $x_{n}=y_{n}$, para cada $n \geq 1, \operatorname{logo} x=y$. Se cada $g_{n}$ é uma geodésica do tipo II), existem duas possibilidades: Se a distância em $\partial P^{\prime}$ entre as extremidades de $\left(g_{n}\right)_{n}$ não estão limitadas inferiormente por uma constante positiva, então $x=y$; caso contrario, as geodésicas $g_{n}$ convergem a uma geodésica $g \in \mathcal{G}$ (junto com as extremidades) entre que contem os pontos $x$ e $y$. O último caso é que cada $g_{n}$ seja o fecho de uma componente $U_{n}$ de $\mathbb{D} \backslash \mathcal{L}$. Para cada $n \geq 1$, seja $\beta_{n}$ o arco geodésico que conecta $x_{n} \operatorname{com} y_{n}$ (assuma que $x_{n} \neq y_{n}$, se não, a conclusão é trivial), e $\gamma_{n}$ a linha geodésica em $\overline{\mathbb{D}}$ que contém $\beta_{n}$. Se a distância entre as extremidades de $\left(\gamma_{n}\right)_{n}$ não estão limitadas inferiormente por uma constante positiva novamente tem-se $x=y$. Se a distância entre as extremidades de $\left(\gamma_{n}\right)_{n}$ estão limitadas inferiormente por uma constante positiva, as geodésicas $\gamma_{n}$ convergem a uma geodésica $\gamma$ contemdo a $x$ e $y$. Se $x=y$ ou $\gamma \in \mathcal{L}$, então $x R_{\mathcal{G}} y$; caso contrario, o arco geodésico $\beta$ que conecta $x$ com $y$ é disjunto da laminação $\mathcal{L}$, logo $x$ e $y$ estão na mesma componente conexa de $\mathbb{D} \backslash \mathcal{L}$.

Portanto $S_{\mathcal{P}}$ é homeomorfo a uma 2-esfera se a dobradura de papel $(P, \mathcal{P})$ é simple. Para provar o recíproco do toerema, suponha que $(P, \mathcal{P})$ não é simple e tome uma dobradura $\left(P^{\prime}, \mathcal{P}^{\prime}\right)$ associada a um árvore de extensão de $\Upsilon_{\mathcal{P}}$ qualquer. Existem dois pareamentos $\left\langle\alpha, \alpha^{\prime}\right\rangle,\left\langle\beta, \beta^{\prime}\right\rangle$ em $\mathcal{P}^{\prime}$ que são vinculados, é dizer, para cada $x \in \stackrel{\alpha}{\alpha}, x^{\prime} \in \stackrel{\circ}{\alpha}^{\prime}, y \in \stackrel{\circ}{\beta}$ e $y^{\prime} \in \stackrel{\circ}{ }^{\prime}, x$ e $x^{\prime}, y$ e $y^{\prime}$ pontos respectivamente pareados, os pares $\left\{x, x^{\prime}\right\},\left\{y, y^{\prime}\right\}$ são vinculados. A identificação de $\alpha \operatorname{com} \alpha^{\prime}$, e de $\beta \operatorname{com} \beta^{\prime}$ vão produzir no espaço de papel $S_{\mathcal{P}}$ pelo menos uma alça, logo $S_{\mathcal{P}}$ não pode ser uma esfera.

Teorema 2.3.9 ([dCH12]). A cicatriz $G_{\mathcal{P}}$ de uma esfera de papel $S_{\mathcal{P}}$ é um dendrito local que pode ser escrito como $G_{\mathcal{P}}=G_{0} \cup \Delta$, onde $G_{0}$ é um dendrito e $\Delta$ é vazio ou, é uma união finita de arcos simples com as seguintes características:

i) As extremidades de cada arco $\delta \in \Delta$ estão em $G_{0}$ e int $\delta \cap G_{0}=\emptyset$.

ii) Se $\delta, \delta^{\prime}$ são dois arcos diferentes de $\Delta$, então int $\delta \cap$ int $\delta^{\prime}=\emptyset$.

Demonstração. Se $(P, \mathcal{P})$ é a dobradura de $S_{\mathcal{P}}$, considere-se um árvore de extensão $T$ de $\Upsilon_{\mathcal{P}}$, pareamentos $\left\langle\alpha_{j}, \alpha_{j}^{\prime}\right\rangle, 1 \leq j \leq k$, associados as arestas de $T$ e a dobradura $\left(P^{\prime}, \mathcal{P}^{\prime}\right)$ obtida ao fazer as identificações dos segmentos $\alpha_{j}$ com $\alpha_{j}^{\prime}$, para cada $j$. Observe-se que cada pareamento $\left\langle\alpha_{j}, \alpha_{j}^{\prime}\right\rangle$ determina um arco $\delta_{j}$ com extremidades em $\partial P^{\prime}$ cujos interiores são dois a dois disjuntos e contidos em int $P^{\prime}$. Se $G_{0}$ é a cicatriz do quociente $P^{\prime} / d^{\mathcal{P}^{\prime}}$, então $G_{\mathcal{P}}=G_{0} \cup \Delta$, sendo $\Delta=\pi_{\mathcal{P}^{\prime}}\left(\cup_{j} \delta_{j}\right)$, pelo qual faltaría provar somente que $G_{0}$ é um dendrito.

Como $\partial P^{\prime}$ é compacto, a cicatriz $G_{0}$ é homeomorfo ao quociente topológico $\partial P^{\prime} / \sim_{\mathcal{P}^{\prime}}$. Por outro lado, sendo $S_{\mathcal{P}}$ uma esfera, a relação $\mathcal{P}^{\prime}$ é desvinculada em $\partial P^{\prime}$ e pelo lema 2.3 .5 , a relação $\sim_{\mathcal{P}^{\prime}}$ 


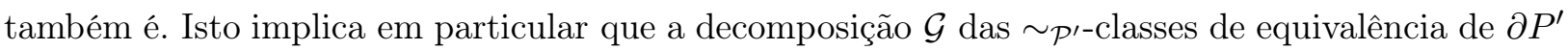
é não-separada. Assím, para demostrar que $G_{0}$ é um dendrito basta provar que $\mathcal{G}$ é dendrítica e usar o Teorema 1.1.20.

Seja $y \notin g \in \mathcal{G}$. Considere-se $A \subset \partial P^{\prime}$ o arco maximal contendo $y$ disjunto de $g$. Essa poligonal é aberta com extremidades $x_{1}, x_{2} \in g$. Como em $\partial P^{\prime}, \sim_{\mathcal{P}^{\prime}}$ é desvinculada, então o arco $A$ é $\sim_{\mathcal{P}^{\prime-}}$ saturado. Suponha por absurdo que não houverem pontos $p$ e $p^{\prime}$ em componenetes distintas de $A \backslash\{y\}$ pareados por algum pareamento de $\mathcal{P}^{\prime}$, logo as poligonals $\left[x_{1}, y\right]$ e $\left[y, x_{2}\right]$ são simples, mas isto implica que $y \in g$ (lema 2.3.3), o qual é uma contradição. Assim existem $p \in\left(x_{1}, y\right)$ e $p^{\prime} \in\left(y, x_{2}\right)$ pareados. Tome um par interior $z, z^{\prime}$ próximo deles tal que $g^{\prime}=\left\{z, z^{\prime}\right\}$ não contém $y$, logo $g^{\prime} \in \mathcal{P}^{\prime}$ separa $y$ de $g$.

\subsection{Pontos irregulares isolados}

Lembre-se que um ponto $q$ num espaço de papel é irregular se ele tem valência $\infty$ ou ele é ponto de acumulação de pontos cônicos, sendo possivel que sejam satisfeitas as duas propriedades anteriores ao mesmo tempo. Na maioria dos exemplos mostrados até o momento, os pontos de valência $\infty$ também são pontos de acumulação de pontos cônicos. Em geral isto não sempre é assim: A superfíce de Chamanara do exemplo 2.2.9 tem um ponto irregular que não é ponto de acumulação de pontos cônicos, de fato, tal espaço não tem nenhum ponto cônico. Outro exemplo de um espaço de papel com um ponto irregular que não é ponto de acumulação de pontos cônicos é o seguinte:

Exemplo 2.4.1. Seja $P=\{x+i t / 0 \leq x, y \leq 1\}$ e $\mathcal{P}$ a dobradura descrita a continuação: O lado superior de $P$ é dobrado pela mitade e os dois lados laterais são colados por uma translação. Em quanto ao lado inferior de $P$, considerese uma sequência infinita de número reais positivos $\left(a_{n}\right)_{n \geq 1}$ tal que $\sum_{n \geq 1} a_{n} \leq \frac{1}{4}$, e uma sequência de pareamentos $\left\langle\alpha_{n}, \alpha_{n}^{\prime}\right\rangle, n \geq 1$, tais que $\left|\left\langle\alpha_{n}, \alpha_{n}^{\prime}\right\rangle\right|=a_{n}$, para todo $n$ como mostra a figura 2.14 .
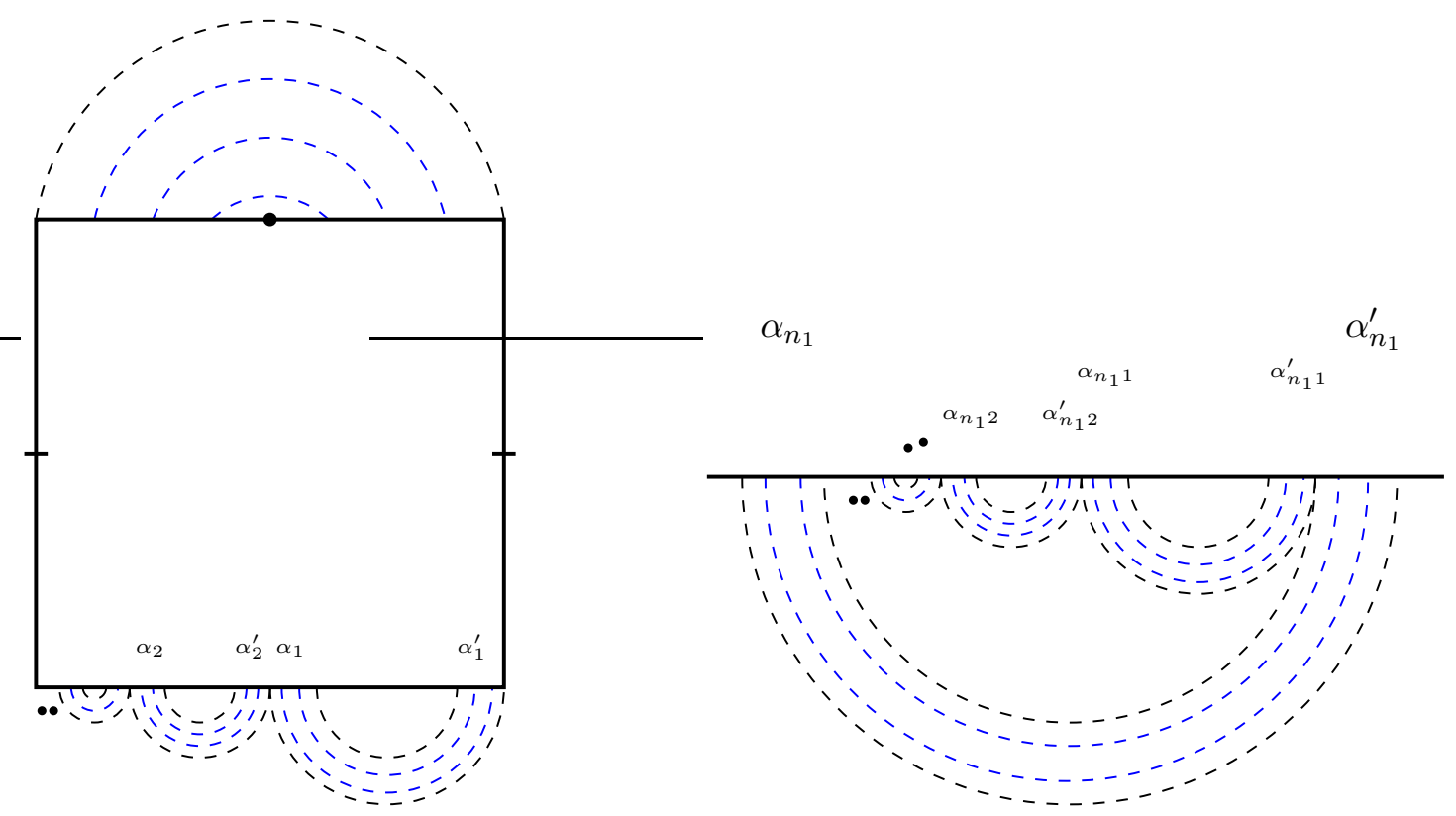

Figura 2.14: Detalhe dos pareamentos no exemplo 2.4.1

$[0,1] \backslash \cup_{n \geq 1}\left(\stackrel{\circ}{\alpha}_{n} \cup \stackrel{\circ}{\alpha}_{n}^{\prime}\right)$ é uma união disjunta de intervalos fechados $\cup_{n \geq 1} I_{n}^{1}$, onde $I_{n}^{1}$ é o intervalo que está entre os intervalos $\alpha_{n}$ e $\alpha_{n}^{\prime}$. Seja $\varphi_{n}:[0,1] \longrightarrow I_{n}^{1}, n \geq 1$, a contração que leva o ponto 0 
na extremidade esquerda de $I_{n}^{1}$ e o ponto 1 na extremidade direita de $I_{n}^{1}$, e defina-se o pareamento $\left\langle\alpha_{n_{1} n_{2}}, \alpha_{n_{1} n_{2}}^{\prime}\right\rangle, n_{1}, n_{2} \geq 1$, onde $\alpha_{n_{1} n_{2}}:=\varphi_{n_{2}}\left(\alpha_{n_{1}}\right), \alpha_{n_{1} n_{2}}^{\prime}:=\varphi_{n_{2}}\left(\alpha_{n_{1}}^{\prime}\right)$, ver figura 2.14 a direita.

Agora $I_{m}^{1} \backslash \cup_{n \geq 1}\left(\stackrel{\circ}{\alpha m}_{n m} \cup \stackrel{\circ}{\alpha m}_{n m}^{\prime}\right)=\cup_{n \geq 1} I_{n}^{2}$, onde $I_{n}^{2}=\varphi_{m}\left(I_{n}^{1}\right)$, para todo $m, n \geq 1$. Completase recursivamente a dobradura de papel $\mathcal{P}$ definiendo-se, para toda sequência finita de naturais positivos $n_{0}, n_{1}, n_{2}, \ldots, n_{k}$, o pareamento $\left\langle\alpha_{n_{1} n_{2} \ldots n_{k}}, \alpha_{n_{1} n_{2} \ldots n_{k}}^{\prime}\right\rangle$, sendo $\alpha_{n_{1} n_{2} \ldots n_{k}}:=\varphi_{n_{k}} \circ \varphi_{n_{k-1}} \circ$ $\ldots \circ \varphi_{n_{1}}\left(\alpha_{n_{0}}\right)$ e $\alpha_{n_{1} n_{2} \ldots n_{k}}^{\prime}:=\varphi_{n_{k}} \circ \varphi_{n_{k-1}} \circ \ldots \circ \varphi_{n_{1}}\left(\alpha_{n_{0}}^{\prime}\right)$.

A esfera de papel $S_{\mathcal{P}}$ associada a $(P, \mathcal{P})$ (observe-se que tal dobradura é simple) tem um único ponto 1-cônico e um único ponto 2-cônico, que são o ponto de dobradura do lado superior e a projeção dos vértices superiores do quadrado respectivamente. No lado inferior do $P$, todas as extremidades dos intervalos $\alpha_{n_{0} n_{1} \ldots n_{k}}, \alpha_{n_{0} n_{1} \ldots n_{k}}^{\prime}, k \geq 0, n_{0}, n_{1}, \ldots, n_{k} \geq 1$, são projetados por $\pi_{\mathcal{P}}$ em algum ponto de $S_{\mathcal{P}}$ de valência $\infty$; o conjunto de todos esses pontos irregulares é infinito e nenhum deles é ponto de acumulção de pontos $k$-cônicos, com $k$ finito. Observe-se que neste caso o conjunto $\mathcal{Q}$ de irregulares é um conjunto perfecto, isto é, todos eles acumulan-se entre si.

A continuação será mostrado que os dois casos acima mencionados são as únicas possibilidades que pode ter um ponto irregular que não seja ponto de acumulação de pontos cônicos

Teorema 2.4.2. Seja $S_{\mathcal{P}}$ um espaço de papel associado a uma dobradura $(P, \mathcal{P})$ e $q \in S_{\mathcal{P}}$ um ponto irregular que não é ponto de acumulação de pontos cônicos. então q é ponto de acumulação de $\infty$-vértices ou $S_{\mathcal{P}}$ contém uma superfície de gênero infinito.

Demonstração. Seja $\mathcal{P}=\left\{\left\langle\alpha_{m}, \alpha_{m}^{\prime}\right\rangle\right\}_{m}$. É fácil ver que $\pi_{\mathcal{P}}^{-1}(q) \cap \bigcup_{m}\left(\alpha_{m} \cup \alpha_{m}^{\prime}\right)$ é infinito. Como a soma dos perímetros dos polígonos de $P$ é finito, existe uma sequência de pareamentos distintos $\left(\left\langle\alpha_{n}, \alpha_{n}^{\prime}\right\rangle\right)_{n \geq 1}$ em $\mathcal{P}, \alpha_{n}=\left[x_{n}, y_{n}\right], \alpha_{n}^{\prime}=\left[x_{n}^{\prime}, y_{n}^{\prime}\right]$, tais que $\left|\left\langle\alpha_{n}, \alpha_{n}^{\prime}\right\rangle\right| \longrightarrow 0$ e $\pi_{\mathcal{P}}\left(x_{n}\right)=\pi_{\mathcal{P}}\left(x_{n}^{\prime}\right)=q$. Não sendo $q$ ponto de acumulação de pontos cônicos pode-se assumir sem perda de generalidade que $q_{n}=\pi_{\mathcal{P}}\left(y_{n}\right)=\pi_{\mathcal{P}}\left(y_{n}^{\prime}\right)$ são pontos irregulares para todo $n \geq 1$, os quais são todos $\infty$-vértices.

Se existe uma sub-sequência $\left(q_{n_{j}}\right)_{j \geq 1}$ tal que $q_{n_{j}} \neq q$, para cada $j \geq 1$, tem-se a primeira afirmação. Se em vez disso $q_{n_{j}}=q$ para todos $j \geq 1$, a família $\left(\pi_{\mathcal{P}}\left(\alpha_{n_{j}}\right)\right)_{j}$ é uma coleção de círculos com um único ponto común, o ponto $q$, cujos diámetros decrecem à 0 . Neste caso, qualquer vizinhança fechada arbitrariamente pequenha de $q$ em $G_{\mathcal{P}}$ deve conter infinitos círculos e, em consequência, tal vizinhança não pode ser um dendrito. Isto implica que a cicatriz $G_{\mathcal{P}}$ não é um dendrito local, pelo qual $S_{\mathcal{P}}$ contém uma superfície de gênero infinito, ver a proposição 2.2 .11 e a proposição 2.2.12.

Um ponto irregular $q$ num espaço de papel $S_{\mathcal{P}}$ dize-se que é isolado, se $q$ é isolado relativo ao conjunto de pontos irregulares $\mathcal{Q}$, isto é, se existe $r>0$ tal que na bola $B_{S_{\mathcal{P}}}(q, r)$ o ponto $q$ é a única irregularidade. Do teorema anterior conclui-se que todo ponto irregular isolado num espaṇo de papel de gênero finito deve ser um ponto de acumulação co conjunto de pontos cônicos $\mathcal{V}$. Neste caso pode-se dizer ainda mais: Tal ponto irregular deve ser ponto de acumulação de pontos 1-cônicos do espaço de papel. Para provar este fato usaremos o seguinte resultado elementar, o qual diz essencialmente que todo caminho simple num dendrito cujo ponto final é um ponto de corte pode ser estendido um pouco mais.

Lema 2.4.3. Seja $G$ um dendrito e $\gamma:[0, T] \longrightarrow G$ um caminho simple tal que $\gamma(T)$ é um ponto de corte de $G$. Então existe $\hat{T}>T$ e um caminho simple $\hat{\gamma}:[0, \hat{T}] \longrightarrow G$ tal que $\hat{\gamma}_{[0, T]}=\gamma$ e $\hat{\gamma}(\hat{T})$ é uma extremidade de $G$. 
Demonstração. Como $\gamma(T)$ separa $G$, considere-se uma componente conexa $C$ de $G \backslash\{\gamma(T)\}$ que não contém $\gamma([0, \gamma(T)) . \bar{C}=C \cup\{\gamma(T)\}$ é um subcontinuum de $G$, logo um dendrito. Se $d$ denota a distância de $G$, existe $x_{0} \in C$ tal que $d\left(\gamma(T), x_{0}\right)=\sup _{x \in C} d(\gamma(T), x)$. Parametrize-se o arco que conecta o ponto $\gamma(T)$ com o ponto $x_{0}$ por um caminho simple $\gamma_{1}:[T, \hat{T}] \longrightarrow \bar{C}$ tal que $\left|\gamma_{1}\right|=$ $d\left(\gamma(T), x_{0}\right)$, logo a concatenação dos caminhos $\hat{\gamma}:=\gamma * \gamma_{1}$ é simple e $\hat{\gamma}_{[0, \hat{T}]}=\gamma$. Falta mostrar que $\gamma(\hat{T})$ é uma extremidade de $G$. Se não, usando-se o argumento acima consegue-se extender o caminho $\gamma_{1}$ para um caminho com extremidades $\gamma(T)$ e $y \in C$, com $d(\gamma(T), y)>d\left(\gamma(T), x_{0}\right)$ o qual é uma contradição.

Teorema 2.4.4. Considere-se um espaço de papel $S_{\mathcal{P}}$ de gênero finito associado a uma dobradura $(P, \mathcal{P})$. Se $q \in S_{\mathcal{P}}$ é um ponto irregular isolado, então q é ponto de acumulação de pontos 1-cônicos.

Demonstração. Seja $G_{\mathcal{P}}$ a cicatriz de $S_{\mathcal{P}}$ e suponha por absurdo que $q \in G_{\mathcal{P}}$ não é ponto de acumulação de pontos 1-cônicos, logo existe $r>0$ tal que a bola fechada $\bar{B}_{G_{\mathcal{P}}}(q, r)$ não contém nem pontos 1-cônicos nem pontos irregulares diferentes de $q$. Tomando $r$ suficientemente pequenho podese assumir também que $\bar{B}_{G_{\mathcal{P}}}(q, r)$ não contém pontos 2-cônicos e que tal bola é um dendrito (Ver proposição 2.2.11). Como $q$ é ponto de acumulação de pontos cônicos (Ver Teorema 2.4.2), existe uma sequência $\left(p_{n}\right)_{n \geq 0} \subset \bar{B}_{G_{\mathcal{P}}}(q, r)$, tal que $d_{G_{\mathcal{P}}}\left(p_{n}, q\right) \searrow 0$, sendo cada $p_{n}$ um ponto $k_{n}$-cônico, com $k_{n} \geq 3$. Além disso, pode-se assumir que algum dos seguintes dois casos é satisfeito:

1) Existe um arco $\delta \subset G_{\mathcal{P}}$ que conecta $p_{0}$ com $q$ e contém a sequência $\left(p_{n}\right)_{n \geq 0}$, sendo estos e o ponto irregular $q$ os únicos pontos não-planares em $\delta$.

2) Existem uma sequência de $\operatorname{arcos}\left(\delta_{n}\right)_{n \geq 0}$ em $G_{\mathcal{P}}$, onde cada arco $\delta_{n}$ conecta o ponto $p_{n}$ com o ponto $q$ e no interior de $\delta_{n}$ não existem pontos não-planares.
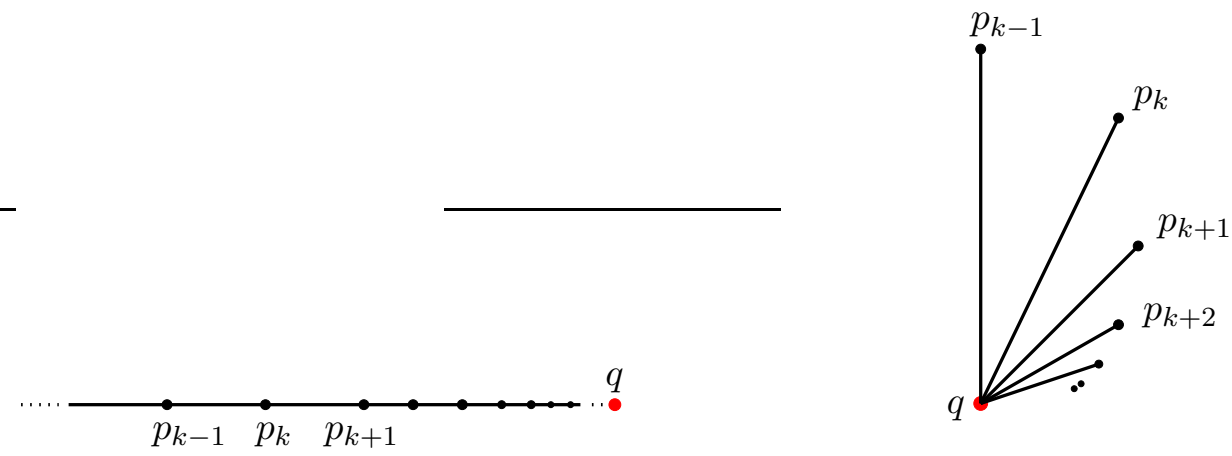

Figura 2.15: Desenho que ilustra o casos 1) e o caso 2) respectivamente.

Na figura 2.15 são mostrados os dois casos mencionados. A prova do teorema será feita só para o caso 1) pois o argumento para o caso 2) é análogo. Observe-se que no caso 1), como $d_{G_{\mathcal{p}}}\left(p_{n}, q\right)>$ $d_{G_{\mathcal{P}}}\left(p_{n+1}, q\right)$, então $\delta \backslash\{q\}$ é a concatenação dos arcos que conecta $p_{n} \operatorname{com} p_{n+1}$, para todo $n \geq 0$, ver a figura 2.15 à esquerda.

Como $k_{n} \geq 3$ para cada $n \geq 0$, existem arcos simples $\gamma_{n} \subset B_{G_{\mathcal{P}}}(q, r)$ com ponto inicial $p_{n}$ tal que $\gamma_{n} \cap \delta=\left\{p_{n}\right\}$. Usando-se o lema 2.4 .3 pode-se assumir que o ponto final do arco $\gamma_{n}$ é uma extremidade do dendrito $\bar{B}_{G_{\mathcal{P}}}(q, r)$ ou o ponto final de $\gamma_{n}$ está em $\mathbb{S}_{G_{\mathcal{P}}}(q, r)$. Procura-se provar que a primeira posibilidade é impossivel. Em efeito, uma extremidade numa cicatriz de um espaço de papel deve ser um ponto 1-cônico ou um ponto irregular, como por hipótese em $\bar{B}_{G_{\mathcal{P}}}(q, r)$ não há pontos 1-cônicos, então o ponto final de $\gamma_{n}$ deve ser uma irregularidade, mas nesse caso, deve ser o ponto $q$. Isto implica que existem dois arcos distintos, um dele é $\gamma_{n}$ e o outro um arco contido 


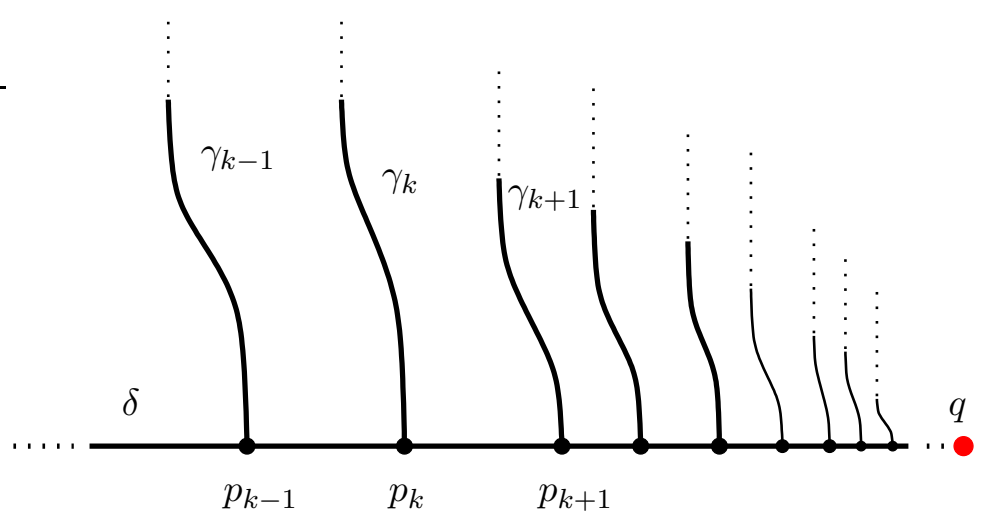

Figura 2.16: Desenho do arco $\delta$ e os arcos $\gamma_{n}$.

em $\delta$, que conectam o ponto $p_{n}$ com o ponto $q$ o qual contradiz que $\bar{B}_{G_{\mathcal{P}}}(q, r)$ seja um dendrito (proposição 1.1.17 ii)). Portanto, para cada $n \geq 1$ o arco $\gamma_{n}$ tem ponto final em $\mathbb{S}_{G_{\mathcal{P}}}(q, r)$.

Usando novamente a proposição 1.1.17 ii), é claro que $\gamma_{n} \cap \gamma_{m}=\emptyset$, se $n \neq m$ : Se não, existe um arco contido em $\delta$ que conecta $p_{n}$ com $p_{m}$, e outro arco contido em $\gamma_{n} \cup \gamma_{m}$ conectando os mesmos pontos. Assim, sendo $m_{G_{\mathcal{P}}}$ uma medida e usando a proposição 2.2.5 iv),

$$
|\partial P|=m_{G_{\mathcal{P}}}\left(G_{\mathcal{P}}\right) \geq m_{G_{\mathcal{P}}}\left(\bigcup_{n \geq 1} \gamma_{n}\right)=\sum_{n \geq 1} m_{G_{\mathcal{P}}}\left(\gamma_{n}\right)=2 \sum_{n \geq 1}\left|\gamma_{n}\right|_{G_{\mathcal{P}}}
$$

Como $d_{G_{\mathcal{P}}}\left(q, \mathbb{S}_{G_{\mathcal{P}}}(q, r)\right)=r$ e $d_{G_{\mathcal{P}}}\left(p_{n}, q\right) \longrightarrow 0$ quando $n \longrightarrow+\infty$, deve existir $\varepsilon>0$ tal que $d_{G_{\mathcal{P}}}\left(p_{n}, \mathbb{S}_{G_{\mathcal{P}}}(q, r)\right)>\varepsilon$, para todo $n \geq 1$. Isto implica que $\left|\gamma_{n}\right|_{G_{\mathcal{P}}} \geq \varepsilon$, para cada $n \geq 1$, pelo qual $|\partial P|=\infty$. Assim, chegamos a uma contradição pelo qual nós concluímos que $q$ tem que ser ponto de acumulação de pontos 1-cônicos.

Para terminar a seção, mostra-se um exemplo de esfera de papel que somente tem pontos planares, pontos irregulares e pontos 3-cônicos. Este exemplo não contradiz o Teorema 2.4.4 pois seu conjunto de irregularidades é um conjunto de Cantor, logo nenhun dele é isolado.

Exemplo 2.4.5. Sejam $a_{n}>0$, tais que $\sum_{n \geq 0} 2^{n+1} a_{n}=1$. Seja $P$ um triângulo equilátero de lado com comprimento igual à um e $\mathcal{P}$ a coleção de pareamentos mostrados na figura 2.17 a esquerda.
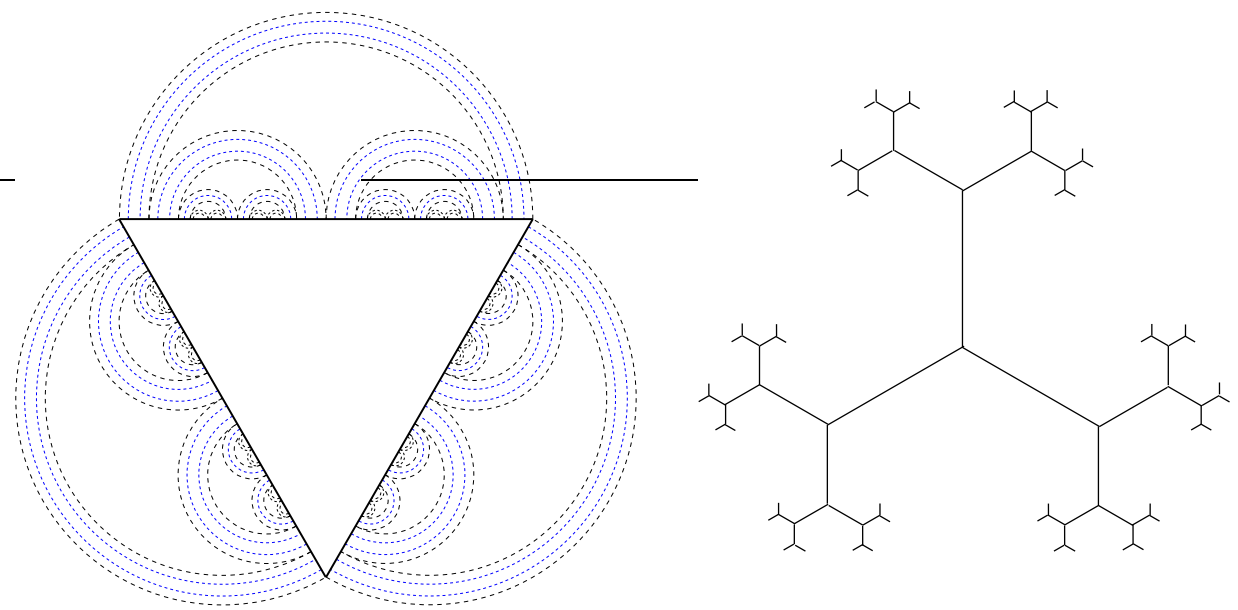

Figura 2.17: Espaço de papel com infinitos pontos 3-cônicos, um Cantor de pontos irregulares e nenhum ponto 1-cônico; ao lado um esboço de sua cicatriz. 
Para entender com mais detalhe a dobradura de papel basta olhar os pareamentos em um lado do triângulo: Começar com um pareamento $\left\langle\alpha, \alpha^{\prime}\right\rangle$ de comprimento $a_{0}$ tal que a extremidade esquerda de $\alpha$ seja um vértice de $P$, a extremidade direita de $\alpha^{\prime}$ seja outro vértice de $P$ e ambas estão contidos num mesmo lado de $P$; seja $B$ o segmento no meio entre $\alpha$ e $\alpha^{\prime}$, e $z_{1}$ o ponto meio de $B$. A continuação considere dois pareamentos $\left\langle\alpha_{0}, \alpha_{0}^{\prime}\right\rangle$ e $\left\langle\alpha_{1}, \alpha_{1}^{\prime}\right\rangle$, ambos com comprimento $\left|\left\langle\alpha_{0}, \alpha_{0}^{\prime}\right\rangle\right|=\left|\left\langle\alpha_{1}, \alpha_{1}^{\prime}\right\rangle\right|=a_{1}$, tais que,

1. $\alpha_{i}, \alpha_{i}^{\prime} \subset B, i=0,1$;

2. A extremidade esquerda de $\alpha_{0}$ e a extremidade direita de $\alpha_{1}^{\prime}$ coincidem com as extremidades esquerda e direita do subsegmento $B$ respectivamente;

3. A extremidade direita de $\alpha_{0}^{\prime}$ e a extremidade esquerda de $\alpha_{1}$ coincidem com o ponto meio $z$.

Denote-se o subsegmento entre $\alpha_{i}$ e $\alpha_{i}^{\prime}$ por $B_{i}$ e seu ponto meio por $z_{i}$, para $i=0,1$; observe-se que com estos dois pareamentos, o ponto $z$ é projetado num ponto 3-cônico. No seguinte paso, considere-se os cuatro pareamentos $\left\langle\alpha_{00}, \alpha_{00}^{\prime}\right\rangle,\left\langle\alpha_{01}, \alpha_{01}^{\prime}\right\rangle,\left\langle\alpha_{10}, \alpha_{10}^{\prime}\right\rangle$ e $\left\langle\alpha_{11}, \alpha_{11}^{\prime}\right\rangle$, todos de comprimento $a_{2}$ descritos a continução:

1. $\alpha_{i_{1} i_{2}}, \alpha_{i_{1} i_{2}}^{\prime} \subset B_{i_{1}}, i_{1}, i_{2}=0,1$;

2. A extremidade esquerda de $\alpha_{00}$ e a extremidade direita de $\alpha_{01}^{\prime}$ coincidem com as extremidades esquerda e direita do subsegmento $B_{0}$ respectivamente;

3. A extremidade esquerda de $\alpha_{10}$ e a extremidade direita de $\alpha_{11}$ coincidem com as extremidades esquerda e direita do subsegmento $B_{1}$ respectivamente;

4. A extremidade direita de $\alpha_{00}^{\prime}$ e a extremidade esquerda de $\alpha_{01}$ coincidem com o ponto meio $z_{0}$

5. A extremidade direita de $\alpha_{10}^{\prime}$ e a extremidade esquerda de $\alpha_{11}^{\prime}$ coincidem com o ponto meio $z_{1}$.

Agora cada ponto $z_{i}, i=0,1$ é projetado num ponto 3-cônico. De maneira similar, denote-se por $B_{i_{1} i_{2}}$ o subsegmento no meio de $\alpha_{i_{1} i_{2}}$ e $\alpha_{i_{1} i_{2}}^{\prime}$ e por $z_{i_{1} i_{2}}$ seu ponto meio, sendo $i_{1} i_{2}=00,01,10,11$. A mesma construção é repetida infinitas vezes até cobrer o lado do triângulo com a coleção de pareamentos $\left\langle\alpha_{i_{1} i_{2} \ldots i_{k}}, \alpha_{i_{1} i_{2} \ldots i_{k}}^{\prime}\right\rangle, i_{1}, i_{2}, \ldots, i_{k}=0,1, k \geq 1$. A figura 2.18 mostra um acercamento de três dos pareamentos descritos acima.

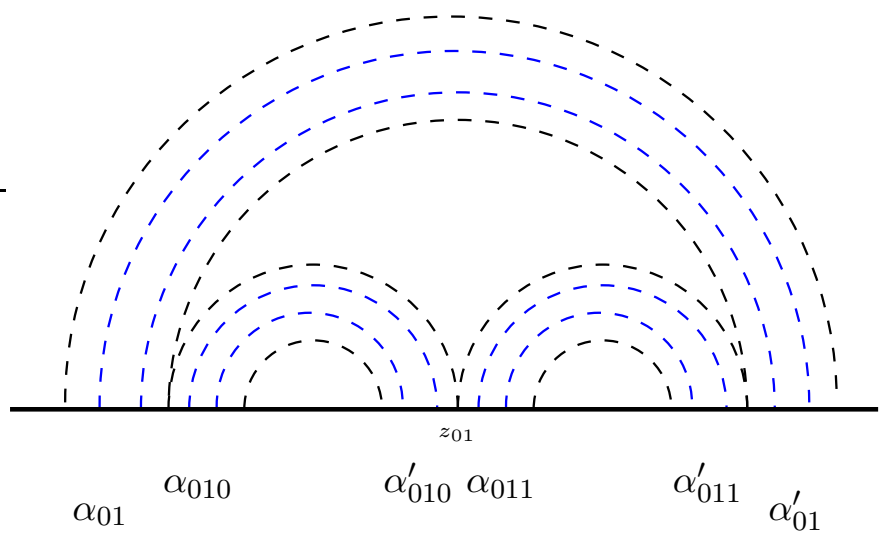

Figura 2.18: Detalhe dos pareamentos $\left\langle\alpha_{01}, \alpha_{01}^{\prime}\right\rangle$ e $\left\langle\alpha_{01 i_{3}}, \alpha_{01 i_{3}}^{\prime}\right\rangle, i_{3}=0,1$.

Assim, dado uma palavra $i_{1} i_{2} \ldots i_{k} \in\{0,1\}^{k}$, tem-se que $p_{i_{1} i_{2} \ldots i_{k}}=\pi_{\mathcal{P}}\left(z_{i_{1} i_{2} \ldots i_{k}}\right)$ é um ponto 3 -cônico do espaço do papel e estos são todos os pontos cônicos de $S_{\mathcal{P}}$. Por outro lado, num lado 
do triângulo e dado $\omega \in\{0,1\}^{\mathbb{N}}$, existe um único ponto $\hat{q}_{\omega}$ na interseção $\bigcap_{k \geq 1} B_{\omega_{\mid[1, k]}}$; a $\pi_{\mathcal{P}}$-projeção deste ponto é o ponto $q_{\omega}$ o qual é o limite da sequência $\left\{p_{\omega_{\mid[1, k]}}\right\}_{k}$, logo um ponto irregular. 


\section{Capítulo 3}

\section{Esfera de papel furada}

Considere-se uma dobradura de papel simple $(P, \mathcal{P})$ e a correspondente esfera de papel $S_{\mathcal{P}}$. Suponha que seu conjunto de pontos irregulares $\mathcal{Q}$ é finito e não vazío, logo o conjunto $\mathcal{V}_{+}$de pontos de curvatura positiva é infinito e acumula-se em cada ponto de $\mathcal{Q}$ (Teorema 2.4.4). Neste capítulo será estudiado a superfície de Riemann $S_{\infty}:=S_{\mathcal{P}} \backslash\left(\mathcal{Q} \cup \mathcal{V}_{+}\right)$com a estrutura complexa induzida como subespaço de $S_{\mathcal{P}} \backslash \mathcal{Q}$; tal espaço será chamado de esfera de papel furada ou simplemente de esfera furada. Sendo $S_{\infty}$ uma superfície do tipo infinito, pode-se assumir sem perda de generalidade que $S_{\infty}=\mathbb{H} / G_{\infty}$, onde $G_{\infty}$ é um modelo Fuchsiano desta superfície. Denote-se por $\lambda_{\infty}(z)|d z|$ à métrica de Poincaré de $S_{\infty}$ e por $\ell_{\infty}(\cdot)$ à função de comprimento de caminhos com esta métrica. Observe-se que em principio, nem o grupo Fuchsiano $G_{\infty}$ nem a métrica de Poincaré de $S_{\infty}$ são conhecidos. Por outro lado é importante resaltar aínda que a estrutura complexa possa ser extendida ao conjunto $\mathcal{Q}$, com o qual $S_{\mathcal{P}}$ é conformemente equivalente à $\widehat{\mathbb{C}}$, a distância esférica e a distância do espaço de papel $d^{\mathcal{P}}$ não são necessariamente comparávels, pelo menos não em nosso conhecimento.

\subsection{Decomposição de $S_{\infty}$}

Suponha que a esfera de papel $S_{\mathcal{P}}$ tem $m$ pontos singulares $q^{1}, q^{2}, \ldots, q^{m}$. Pelo Teorema 2.4.4, cada ponto $q^{j}$ é ponto de acumulação do conjunto de pontos 1-cônicos de $S_{\mathcal{P}}$, logo pode-se escrever o conjunto $\mathcal{V}_{+}$como uma união disjunta finita $\mathcal{V}_{+}=\mathcal{V}_{+}^{1} \sqcup \mathcal{V}_{+}^{2} \sqcup \ldots \sqcup \mathcal{V}_{+}^{m}$, onde cada ponto $q^{j}$ é o único ponto de acumulação do conjunto $\mathcal{V}_{+}^{j}, 1 \leq j \leq m$. Em conseqüência cada conjunto $\mathcal{V}_{+}^{j}$ pode ser ordenado como uma sequência $\mathcal{V}_{+}^{j}=\left\{p_{1}^{j}, p_{2}^{j}, \ldots\right\}$, de forma que $\lim _{k \rightarrow+\infty} p_{k}^{j}=q^{j}$, para todo $j=1, \ldots, m$. Nesta primeira seção será descrita uma multicurva $\Gamma_{\infty} \in S_{\infty}$ relativamente natural que separa à superfície em calças justas; a multicurva dependerá essencialmente da partição do conjunto $\mathcal{V}_{+}$e do ordenamento feito em cada um dos subconjuntos da partição, com o qual assumiremos sempre que estos já foram fixados como está explicado acima.

Suponha-se inicialmente que o número de pontos irregulares é $m \geq 3$. É possível escolher a partição $\mathcal{V}_{+}=\bigsqcup_{1 \leq j \leq m} \mathcal{V}_{+}^{j}$ de maneira que, se $D_{0}^{j}$ denota o menor sub-continuum em $G_{\mathcal{P}}$ que contém $\left\{q^{j}\right\} \cup \mathcal{V}_{+}^{j}$, então $D_{0}^{j_{1}} \cap D_{0}^{j_{2}}=\emptyset$ sempre que $j_{1} \neq j_{2}$ : Tome-se $r>0$ tal que as bolas fechadas em $G_{\mathcal{P}}, \bar{B}_{G_{\mathcal{P}}}\left(q^{1}, r\right), \ldots, \bar{B}_{G_{\mathcal{P}}}\left(q^{m}, r\right)$ sejam dendritos dois a dois disjuntos; como fora delas só ficam um número finito de pontos de $\mathcal{V}_{+}$, basta distribuir estos pontos conectando-os com um arco em $G_{\mathcal{P}}$ à alguma dessas bolas até obter a partição procurada. Dado qualquer $1 \leq j \leq m$ e $k \geq 1$, seja $D_{k}^{j}$ o menor subcontinuum em $G_{\mathcal{P}}$ que contém o subconjunto $\left\{q^{j}\right\} \cup\left\{p_{k+1}^{j}, p_{k+2}^{j}, \ldots\right\}$, e $E_{k}^{j}$ o resto dos pontos em $\mathcal{Q} \cup \mathcal{V}_{+}$, é dizer, $E_{k}^{j}=\overline{\mathcal{V}} \backslash D_{k}^{j}$. Claramente $D_{0}^{j} \supset D_{1}^{j} \supset D_{2}^{j} \supset \ldots$, pelo qual $D_{k_{1}}^{j_{1}} \cap D_{k_{2}}^{j_{2}}=\emptyset$ se $j_{1} \neq j_{2}, k_{1}, k_{2} \geq 0$.

A ideia agora consiste em usar cada sub-continuum $D_{k}^{j}$ para determinar uma única curva geodésica $\gamma_{k}^{j}$ na superfície $S_{\infty}$, para todo $j, k$. Sendo $D_{k}^{j}$ um conjunto conexo, pela Proposição 1.3.8 existe uma única curva fechada simples $\hat{\gamma}_{k}^{j} \subset S_{\mathcal{P}}$ que separa os conjuntos $D_{k}^{j}$ e $E_{k}^{j}$ a qual é uma 
geodésica na superfície $S_{\mathcal{P}} \backslash\left(D_{k}^{j} \cup E_{k}^{j}\right)$. Como $\hat{\gamma}_{k}^{j} \subset S_{\infty}$, tome-se $\gamma_{k}^{j}$ definida como a única geodésica em $S_{\infty}$ na classe de homotopía de $\hat{\gamma}_{k}^{j}$, com o qual esta será também fechada e simples. Observe-se que o Lema 1.3.9 implica que a sequência de geodésicas $\left(\gamma_{k}^{j}\right)_{k \geq 0}$ são concêntricas no sentido que, para todo $k \geq 0$, as curvas $\gamma_{n}^{j}, n>k$, estão na mesma componente de $S_{\mathcal{P}} \backslash \gamma_{k}^{j}$. A figura 3.1 mostra um esboço das geodésicas $\gamma_{k}^{j}$ e $\gamma_{k+1}^{j}$ em um caso particular. Por construção, as curvas $\gamma_{0}^{j}, \gamma_{0}^{j}, \ldots, \gamma_{0}^{j}$ são disjuntas duas a duas, logo o mesmo acontece com a família de curvas $\left\{\gamma_{k}^{j} / 1 \leq j \leq m, k \geq 0\right\}$. Para finalizar, a superfície $S^{0}=S_{\mathcal{P}} \backslash \bigcup_{j=1}^{m} D_{0}^{j}$ é do tipo $(0,0, m)$, logo pose-se escolher à vontade $m-3$ curvas $\hat{\gamma}_{1}^{0}, \hat{\gamma}_{2}^{0}, \ldots, \hat{\gamma}_{m-3}^{0}$ que separem à $S^{0}$ em calças, depois tome-se as geodésicas $\gamma_{1}^{0}, \ldots, \gamma_{m-3}^{0}$ em $S_{\infty}$ nas respectivas classes de homotopía. Tome-se $\Gamma_{\infty}$ como a coleção de geodésicas acima descrita, logo esta multicurva separa a esfera furada $S_{\infty}$ em calças.
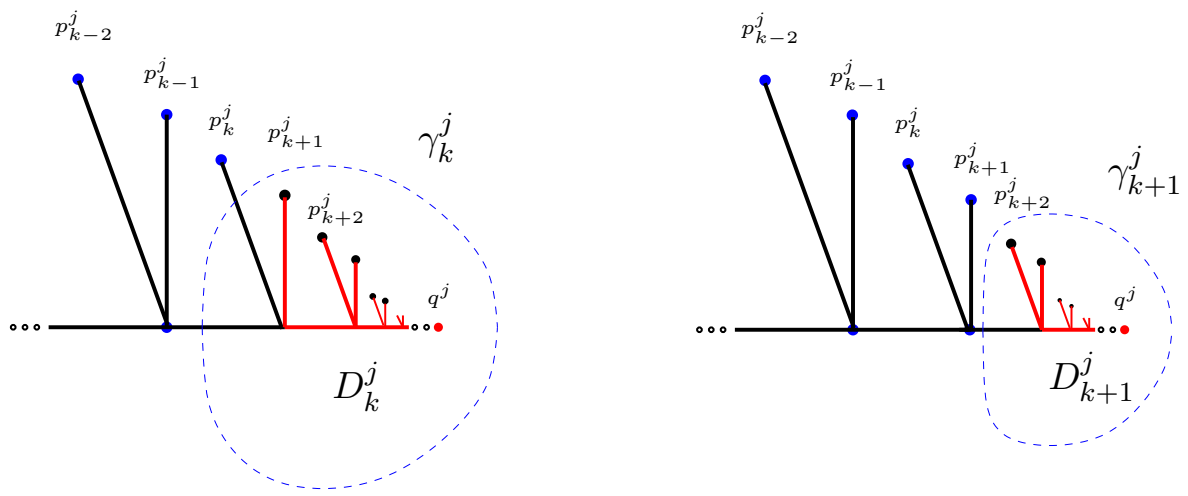

Figura 3.1: Construção das geodésicas $\gamma_{k}^{j}$.

Observe-se que foi reproduzido a prova da Proposição 1.4.11 para esta superfície: O espaço dos fins de $S_{\infty}$ é homeomorfo ao subespaço $\overline{\mathcal{V}}_{+}=\mathcal{V}_{+} \cup \mathcal{Q}$, sendo cada fin finito um furo o qual a sua vez é identificado com cada ponto de $\mathcal{V}_{+}$, e sendo cada fin infinito da superfície identificado com cada ponto irregular $q^{1}, \ldots, q^{m}$. Assim conclui-se que a esfera de papel furada com $m$ pontos irregulares é uma superfície gaita de fole justa de ordem $m$. Escolha-se o modelo Fuchsiano $G_{\infty}$ de $S_{\infty}$ como na definição 1.4.4, de maneira que cada elemento $g_{k}^{j} \in G_{\infty}$ corresponda com a geodésica $\gamma_{k}^{j}$, para todo par de índices $j, k$.
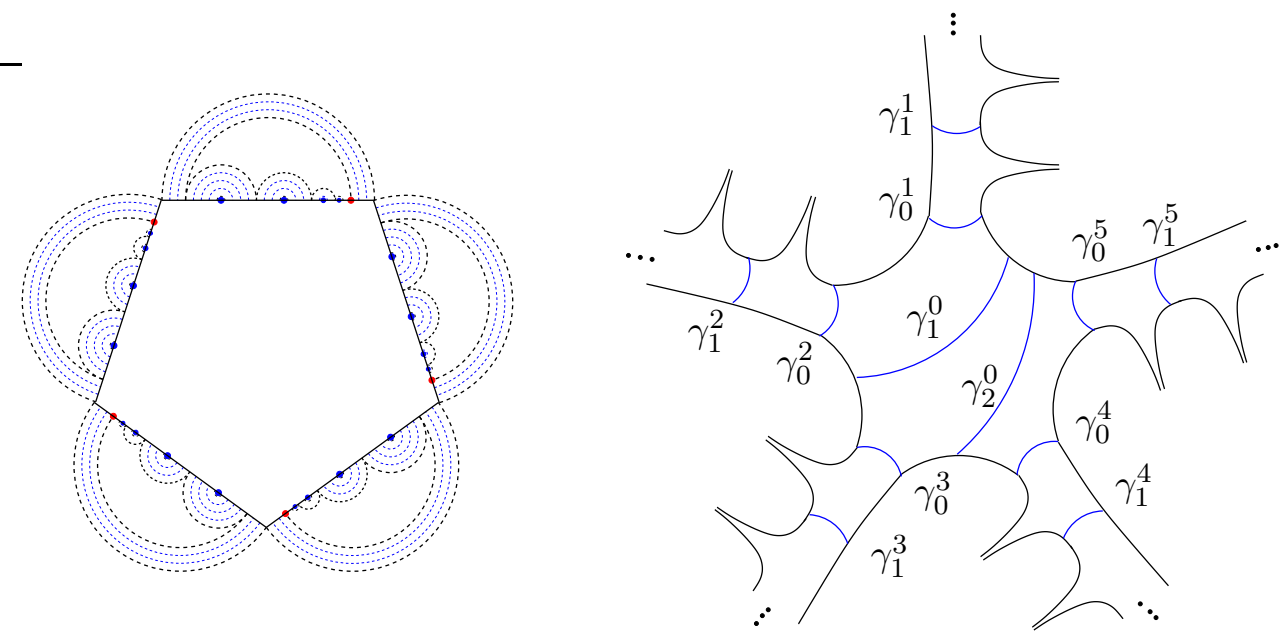

Figura 3.2: Uma esfera de papel furada com 5 fins infinitos

Quando a esfera de papel $S_{\mathcal{P}}$ tem um ou dois pontos irregulares a construção da multicurva $\Gamma_{\infty}$ será similar ao caso anterior com $m \geq 3$ pontos irregulares, a diferença está em que não será 
necessario particionar o conjunto de pontos $\mathcal{V}_{+}$: Ordene-se este conjunto como uma sequência $\left\{p_{n}\right\}_{n}$ de forma que $\mathcal{Q}=\left\{\lim _{n \rightarrow+\infty} p_{n}\right\}$, se $m=1$ e $\mathcal{Q}=\left\{\lim _{n \rightarrow+\infty} p_{n}, \lim _{n \rightarrow-\infty} p_{n}\right\}$, se $m=2$, logo defina-se $D_{k}$ como o menor subcontinuum em $G_{\mathcal{P}}$ que contém $\overline{\left\{p_{n}\right\}_{n>k}}$ e $E_{k}$ o complemento de $D_{k}$ em $\overline{\mathcal{V}}_{+}$(No caso $m=1$ será conveniente escrever $\mathcal{V}_{+}$como uma sequência que comença no ponto $\left.p_{-1}, p_{0}, p_{1} \ldots\right)$. A multicurva $\Gamma_{\infty}=\left\{\gamma_{k}\right\}_{k}$ construida de maneira similar ao caso $m \geq 3$ usando os conjuntos $D_{k}$ e $E_{k}$, separará em calças a esfera furada $S_{\infty}$. Novamente esta será uma gaita de fole de ordem um e dois respectivamente, é dizer uma flauta justa se existe um único ponto irregular ou uma biflauta justa se existem dois pontos irregulares, ver figura 3.3.
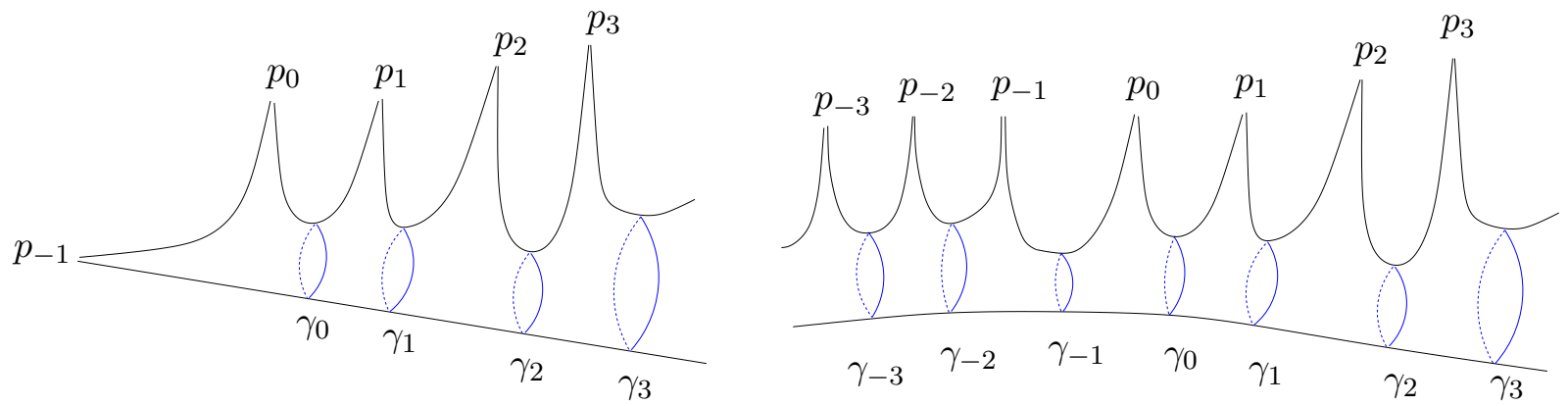

Figura 3.3: Esfera de papel furado com um e dois fins infinitos respectivamente.

Em conclusão, toda esfera de papel furada será uma superfície gaita de fole justa cujo ordem é igual ao número de pontos irregulares do espaço de papel $S_{\mathcal{P}}$. Uma pergunta interesante sería saber se o recíproco vale, isto é, dada uma superfície gaita de fole justa $S$ de ordem $m$ qualquer, ela pode ser realizada por alguma esfera de papel furada com $m$ irregularidades? E se a resposta for negativa, que condições precisa satisfazer a gaita de fole para ser realizada por uma esfera de papel furada? ou, existe uma esfera furada $S_{\infty}$ e uma aplicação quaseconforme $f: S_{\infty} \longrightarrow S$ ? Espera-se responder algumas destas perguntas numa futura pesquisa. Por enquanto, um primeiro passo consiste em entender os possívels comprimentos hiperbólicos das curvas geodésicas na família $\Gamma_{\infty}$ que faz a decomposição em calças da mesma.

\subsection{Modelo base}

A continuação serão calculadas estimativas superiores para os comprimentos hiperbólicos das curvas $\Gamma_{\infty}$ de uma esfera de papel furada particular que chamaremos de modelo base. A razão de iniciar com este exemplo particular deve-se a simplicidade deste modelo o qual permitirá entender facilmente os argumentos usados para o calculo das mesmas estimativas superiores numa esfera furada geral. Este modelo base corresponde a uma esfera de papel com um único ponto irregular o qual é um $\infty$-vértice e é possivelmente o exemplo mais básico de um espaço de papel com irregularidades.

Comenzamos então com a descrição do espaço de papel do modelo base. Considere-se uma sequência decrecente $\left(a_{k}\right)_{k \geq 1}$ de números reais positivos tal que

$$
\sum_{k \geq 1} a_{k}=\frac{L}{2}<+\infty
$$

Seja $P$ o quadrado cujos vértices são os pontos $0, L, i \cdot L$ e $L+i \cdot L$, e $\mathcal{P}$ a coleção de pareamentos que consiste num pareamento $\left\langle\alpha_{-1}, \alpha_{-1}^{\prime}\right\rangle$ que dobra o lado superior do quadrado $P$ pela mitade, outro pareamento $\left\langle\alpha_{0}, \alpha_{0}^{\prime}\right\rangle$ que identifica os lados verticais do $P$ pela translação $z \longmapsto z+L$, e a familía de pareamentos $\left\{\left\langle\alpha_{k}, \alpha_{k}^{\prime}\right\rangle\right\}_{k \geq 1}$ contidas no lado inferior de $P$, com as seguintes características:

i) Para cada $k \geq 1,\left|\left\langle\alpha_{k}, \alpha_{k}^{\prime}\right\rangle\right|=a_{k}$. 
ii) Para cada $k \geq 1$, a extremidade esquerda de $\alpha_{k}$ coincide com a extremidade direita de $\alpha_{k}^{\prime}$, de modo que $\alpha_{k}^{\prime} \cup \alpha_{k}$ é um subsegmento do lado inferior de $P$.

iii) Se $k_{1}<k_{2}$, o segmento $\alpha_{k_{2}}^{\prime} \cup \alpha_{k_{2}}$ está na esquerda do segmento $\alpha_{k_{1}}^{\prime} \cup \alpha_{k_{1}}$.

O modelo base corresponde à esfera de papel furada do $S_{\mathcal{P}}$. A figura 3.4 mostra a dobradura de papel acima descrita e sua respectiva cicatriz. Observe-se que a união de todos os segmentos $\alpha_{k}^{\prime} \cup \alpha_{k}, k \geq 1$, cobre todo o lado inferior do $P$ exceto no vértice 0 . $\mathrm{O}$ conjunto $\mathcal{V}_{+}$consiste em todos os pontos 1-cônicos $p_{k}, k \geq 1$, correspondentes as dobradoras feitas no lado inferior do quadrado, o ponto 1 cônico $p_{-1}$ correspondente a dobradura do lado superior e um ponto 2-cônico $p_{0}$ o qual é a $\pi_{\mathcal{P}}$-projeção dos vértices superiores do $P$ (observe-se que este ponto tem curvatura igual a $\pi$ como os outros). A irregularidade é o ponto $q=\pi_{\mathcal{P}}(0)$; observe-se que $\pi_{\mathcal{P}}^{-1}(q)=\{0\} \cup\left\{c_{k}\right\}_{k \geq 0}$, onde $c_{k}=2 \sum_{n>k} a_{n}$. Por outro lado já o conjunto $\mathcal{V}_{+}$foi ordenado de forma que $\lim _{k \rightarrow+\infty} p_{k}=q$, pois $d^{\mathcal{P}}\left(p_{k}, q\right)=a_{k}$.
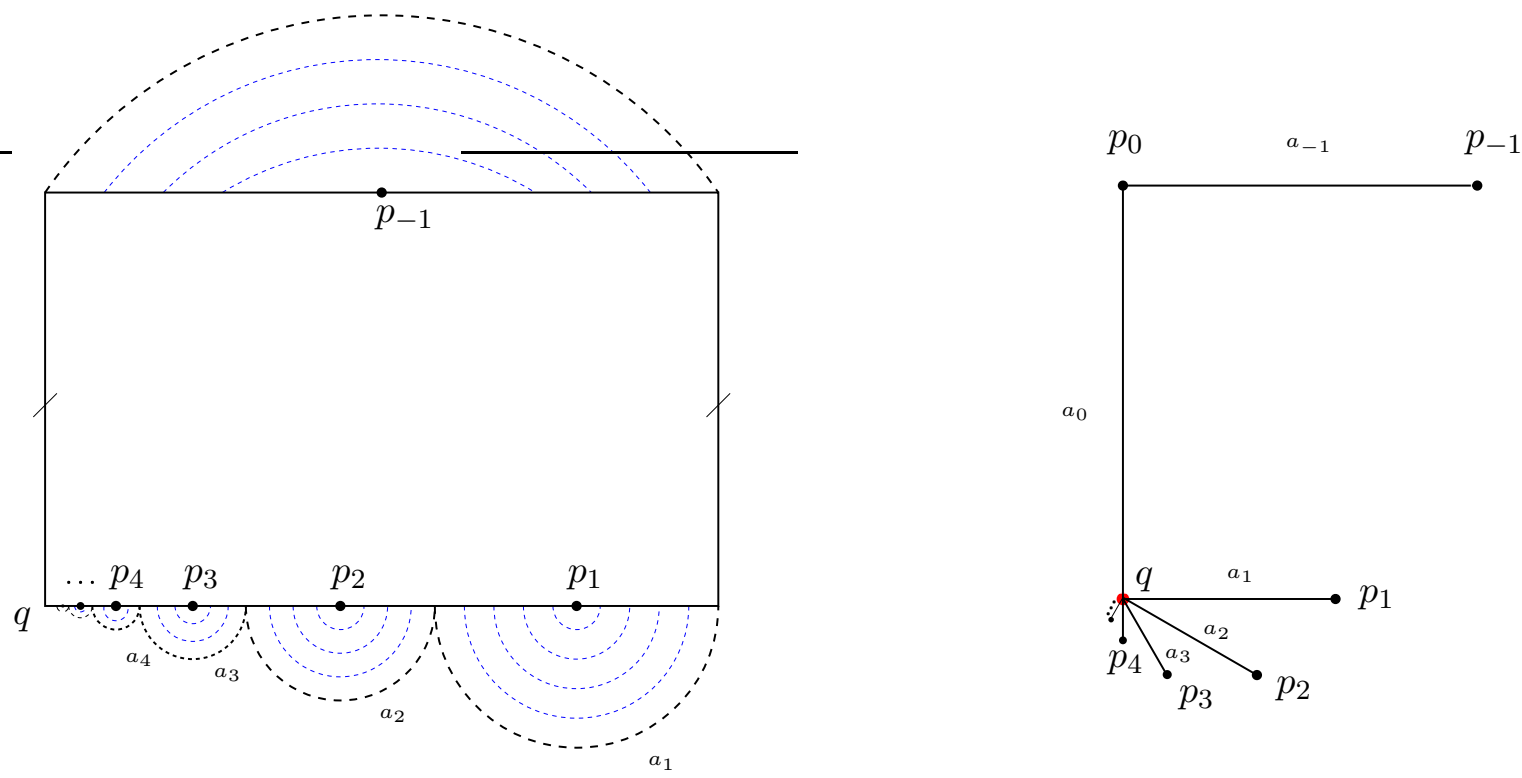

Figura 3.4: Esfera de papel do modelo base e sua cicatriz

Considere-se a multicurva $\Gamma=\left(\gamma_{k}\right)_{k \geq 0}$ que separa à $S_{\infty}$ numa sequência de calças como foi explicado na seção anterior. Neste caso cada geodésica $\gamma_{k}$ separa os conjuntos de furos $\left\{p_{-1}, p_{0}, p_{1}, \ldots, p_{k}\right\}$ e $\{q\} \cup\left\{p_{k+1}, p_{k+2}, \ldots\right\}$. A figura 3.5 mostra um esboço de uma destas geodésicas no espaço $S_{\infty}$.

Procuramos estimar o comprimento hiperbólico $\ell_{\infty}\left(\gamma_{k}\right)$ para cada $k \geq 0$. Lembre-se que não são conhecidas as representações matriciais dos elementos $g_{k} \in G_{\infty}$, logo não é possível usar a Proposição 1.3.1 iv), para calcular exatamente qual é o valos deste comprimento. A continuação será explicado o argumento que servirá para conseguir as estimativas de $\ell_{\infty}\left(\gamma_{k}\right)$; este argumento possívelmente é devido à L. Ahlfors ou A. Beurling. Lembre-se que num anel de módulo finito $A$ existe uma única geodésica $\alpha_{A}$ cujo comprimento é $\ell_{A}\left(\alpha_{A}\right)=\frac{\pi}{\operatorname{Mod} A}$.

Definição 3.2.1. Seja $S$ uma superfície hiperbólica e $A \hookrightarrow S$ uma subsuperfície annular mergulhada em $S$. Se $\gamma \subset S$ é uma curva fechada dize-se que o anel $A$ é redutivel à $\gamma$ se as curvas $\gamma$ e a curva $\alpha_{A}$ são livremente homotópicas em $S$.

Como numa superfície hiperbólica $S$ uma geodésica fechada sempre realiza o menor comprimento na sua classe de homotopía, se $A \subset S$ é um anel redutível à geodésica fechada simples $\gamma \subset S$, então $\ell_{S}(\gamma) \leq \ell_{S}\left(\alpha_{A}\right)$. Sendo neste caso $A$ uma subsuperfície de $S$, a Proposição 1.2.23 implica que 

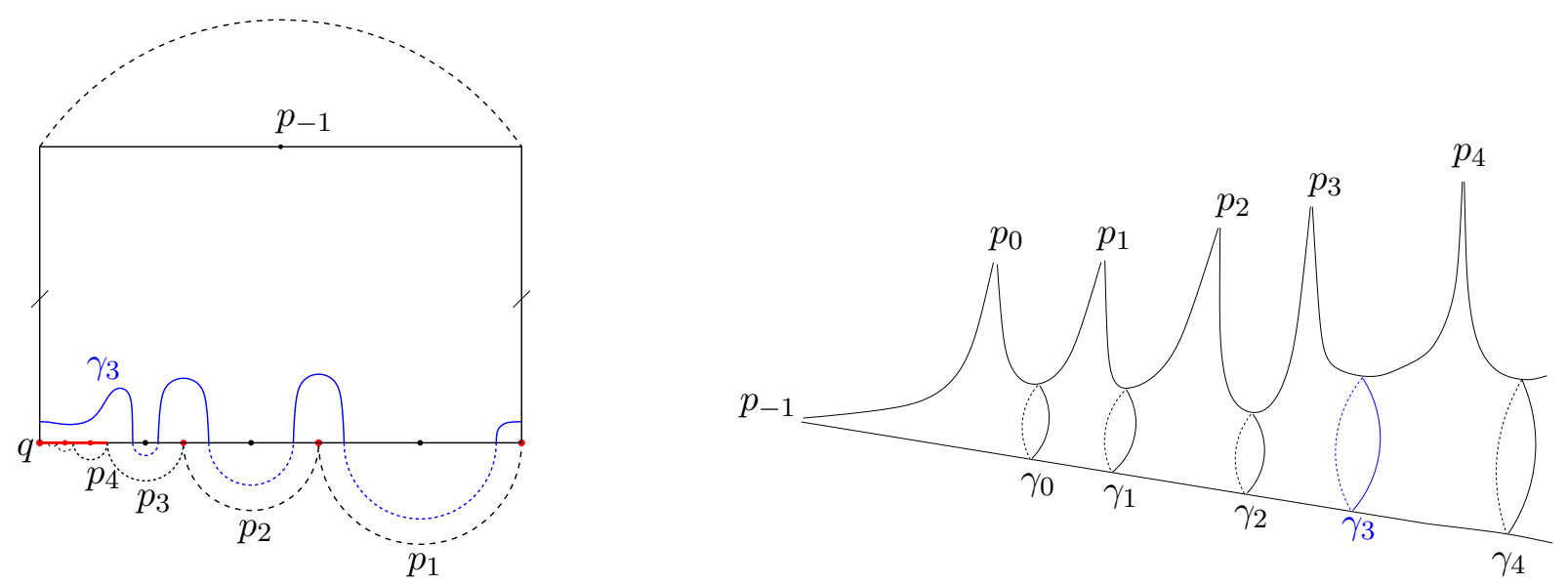

Figura 3.5: Possivel desenho da geodésica $\gamma_{3}$ em azul. No desenho a esquerda pode-se ver em vermelho as componentes de $\pi_{\mathcal{P}}^{-1}\left(D_{3}\right)$

$\ell_{S}\left(\alpha_{A}\right) \leq \ell_{A}\left(\alpha_{A}\right)$. Usando agora a Proposição 1.3.3, considere-se uma densidade Boreliana $\rho \in m_{A}$ (Lembre-se que $\mathcal{A}_{\rho}(A)$ denota a $\rho$-área de $A$ e $\mathcal{L}_{\rho}(A)$ denota a $\rho$-distância entre as componentes de fronteira de $A)$, logo,

$$
\operatorname{Mod} A \geq \frac{\mathcal{L}_{\rho}(A)^{2}}{\mathcal{A}_{\rho}(A)}
$$

Combinando as três desigualdades anteriores hemos demostrado o seguinte resultado.

Lema 3.2.2. Sejam $S$ uma superfície hiperbólica e $\gamma \subset S$ uma geodésica fechada simples. Se $A \subset S$ é um anel redutivel à $\gamma$ e se $\rho(z)|d z|$ é uma densidade Boreliana em A, então,

$$
\ell_{S}(\gamma) \leq \pi \cdot \frac{\mathcal{A}_{\rho}(A)}{\mathcal{L}_{\rho}(A)^{2}}
$$

Voltando ao modelo base $S_{\infty}$ precisa-se conseguir para cada $k \geq 0$, um subanel $A_{k} \subset S_{\infty}$ e uma densidade $\rho_{k} \in m_{A_{k}}$, ambas convenientes em algum sentido, de forma que o anel $A_{k}$ seja redutível à geodésica $\gamma_{k}$, e assim fazer úso da desigualdade 3.1 do lema acima. Aqui tal vez a parte delicada seja a escolha da densidade Boreliana de forma que a estimativa seja "boa"e ao mesmo tempo fácil de calcular. Por exemplo, a densidade Euclideana $\rho_{e}(z)|d z|$ definida sobre um espaço de papel $S_{\mathcal{P}}$ por $\rho_{e}(z)=1$, para todo $z \in S_{\mathcal{P}}$, resulta ser a densidade mais natural num espaço de papel pela seguinte razão: esta indúz a distância $d^{\mathcal{P}}$ do $S_{\mathcal{P}}$ e para todo Boreliano $A \subset S_{\mathcal{P}}$, a $\rho_{e}$-área de $A$ é a área Euclideana de $\pi_{\mathcal{P}}^{-1}(A) \subset P$. Para a primeira curva de $\Gamma_{\infty}$, a geodésica $\gamma_{0}$, considere-se a banda $\hat{A}_{0}=\left\{z \in P / \frac{L-s}{2}<\operatorname{Im} z<\frac{L+s}{2}\right\}$, onde $s \in(0, L]$, logo o anel $A_{0}:=\pi_{\mathcal{P}}\left(\hat{A}_{0}\right)$ (Observe-se que $A_{0}$ separa $p_{-1}$ e $p_{0}$ dos outros furos) é redutível à $\gamma_{0}$, com o qual tem-se $\mathcal{A}_{\rho_{e}}\left(A_{0}\right)=s \cdot L$ e $\mathcal{L}_{\rho_{e}}\left(A_{0}\right)=s$. Logo a desigualdade 3.1 implica que $\ell_{\infty}\left(\gamma_{0}\right) \leq \pi \frac{L}{s}$; em particular para $s=L, \ell_{\infty}\left(\gamma_{0}\right) \leq \pi$.

Para cada $k \geq 1$, observe-se que o conjunto $D_{k}$ definido como o menor sub-continuum em $G_{\mathcal{P}}$ que contém os pontos $q, p_{k+1}, p_{k+2}, \ldots$, é a união dos arcos em $G_{\mathcal{P}}$ que conectam cada ponto 1cônico $p_{n}$ com o ponto irregular $q$, para $n>k$, $\log$ o $\pi_{\mathcal{P}}^{-1}\left(D_{k}\right)=\left[0, c_{k}\right] \cup\left\{c_{k-1}, \ldots, c_{1}, c_{0}\right\}$. Por outro lado $d^{\mathcal{P}}\left(E_{k}, D_{k}\right)=d^{\mathcal{P}}\left(p_{k}, q\right)=a_{k}$, assim que, para $0<r \leq a_{k}$, o anel $A_{k}=B_{d^{\mathcal{P}}}\left(D_{k}, r\right) \backslash D_{k}$ separa ambos conjuntos, sendo assim o anel $A_{k}$ é redutível à $\gamma_{k}$. Usando novamente a densidade $\rho_{e}$, tem-se $\mathcal{L}_{\rho_{e}}\left(A_{k}\right)=r$ e, como $\pi_{\mathcal{P}}^{-1}\left(A_{k}\right)=R \cup C_{k} \cup C_{k-1} \cup \ldots C_{1} \cup C_{0}$, onde $R=\left[0, c_{k}\right] \times(0, r)$, $C_{j}=B_{P}\left(c_{j}, r\right) \backslash\left\{c_{j}\right\}$ para $j=0,1, \ldots, k-1$, e $C_{k}=B_{P}\left(c_{k}, r\right) \cap\left\{z \in P / \operatorname{Re} z>c_{k}\right\}$, então 


$$
\begin{aligned}
\mathcal{A}_{\rho_{e}}\left(A_{k}\right) & =\mathcal{A}_{\text {eucl. }}(R)+\sum_{j=0}^{k} \mathcal{A}_{\text {eucl. }}\left(C_{j}\right) \\
& =c_{k} \cdot r+k \cdot \frac{\pi}{2} \cdot r^{2}
\end{aligned}
$$

Logo usando novamente 3.1, tem-se $\ell_{\infty}\left(\gamma_{k}\right) \leq M_{k}=\pi\left(\frac{c_{k}}{r}+k \frac{\pi}{2}\right)$, para todo $k \geq 1$. Aínda foi obtido uma estimativa para $\ell_{\infty}\left(\gamma_{k}\right)$ com a densidade Euclideana $\rho_{e}$, é claro que as estimativas são insuficientes para entender o comportamento asintótico da sequência de comprimentos $\left(\ell_{\infty}\left(\gamma_{k}\right)\right)_{k>1}$. Em efeito $M_{k} \longrightarrow+\infty$ quando $k \rightarrow+\infty$, mas isto não implica necessariamente que os comprimentos das curvas $\gamma_{k}$ crescem também junto com as cotas $M_{k}$. O problema aquí está no número de peças circulares $C_{0}, \ldots, C_{k}$, os quais crescem proporcionalmente ao índice $k$; aínda os raios das peças sejam cada vez menor, a relação área/raio da desigualdade 3.1 faz que o valor $M_{k}$ seja também proporcional à $k$.

Para melhorar as estimativas das curvas $\gamma_{k}$, procuraremos densidades que de algum jeito controlem o crescimento das componentes do conjunto $\pi_{\mathcal{P}}^{-1}\left(D_{k}\right)$ para evitar um resultado similar as estimativas feitas com a densidade $\rho_{e}$. Considere-se $k \geq 1$ e um número real $t>1$ arbitrario. Tome-se agora o anel $A_{k}^{t}$, dado por

$$
A_{k}^{t}:=B_{d^{\mathcal{P}}}\left(D_{k}, a_{k}\right) \backslash \bar{B}_{d^{\mathcal{P}}}\left(D_{k}, \frac{a_{k}}{t^{k}}\right),
$$

o qual novamente é redutível à geodésica $\gamma_{k}$. Neste caso $\pi_{\mathcal{P}}^{-1}\left(A_{k}^{t}\right)=R^{t} \cup \bigcup_{j=0}^{k} C_{j}^{t}$, sendo

$$
\begin{aligned}
R^{t} & =\left[0, c_{k}\right] \times\left(\frac{a_{k}}{t^{k}}, a_{k}\right) \\
C_{0}^{t} & =\left\{r e^{i \theta} / \frac{a_{k}}{t^{k}}<r<a_{k}, \quad \frac{\pi}{2} \leq \theta \leq \pi\right\} \\
C_{k}^{t} & =\left\{r e^{i \theta} / \frac{a_{k}}{t^{k}}<r<a_{k}, 0 \leq \theta \leq \frac{\pi}{2}\right\} \\
C_{j}^{t} & =\left\{r e^{i \theta} / \frac{a_{k}}{t^{k}}<r<a_{k}, 0 \leq \theta \leq \pi\right\}, j=1,2, \ldots, k-1 .
\end{aligned}
$$

Na figura 3.6 é mostrado por exemplo um desenho do anel $A_{3}^{t}$

Em vez de usar a densidade $\rho_{e}$ em $A_{k}^{t}$, tome-se a função $\rho_{k}^{t}: A_{k}^{t} \longrightarrow[0,+\infty)$ definida por:

$$
\rho_{k}^{t}(z):=\left\{\begin{array}{lll}
\frac{1}{d^{\mathcal{P}}\left(z, D_{k}\right)} ; & \text { se } & \pi_{\mathcal{P}}^{-1}(z) \in \bigcup_{j=0}^{k} C_{k}^{t} \\
\frac{k \cdot \log t}{a_{k}\left(1-\frac{1}{t^{k}}\right)} ; & \text { se } & \pi_{\mathcal{P}}^{-1}(z) \in R^{t}
\end{array}\right.
$$

Observe-se que a densidade $\rho_{k}^{t}$ é somente descontínua na fronteira relativa ao anel da peça retangular $R^{t}$, pelo qual $\rho_{k}^{t}$ é uma função Borel-mensurável. A ventagem da densidade $\rho_{k}^{t}$ está nas regiões circulares $C_{j}^{t}$ a fórmula $\frac{1}{d^{\mathcal{P}}\left(z, D_{k}\right)}$ pois alí a relação $\rho_{k}^{t}$-área/ $\rho_{k}^{t}$-largura ao quadrado é proporcional à $\frac{1}{k}$. Na parte retangular $R^{t}$ a densidade $\rho_{k}^{t}$ é a densidade plana multiplicada por o fator $\frac{k \cdot \log t}{a_{k}\left(1-\frac{1}{t^{k}}\right)}$; a escolha deste fator particular será esclarecido na prova do seguinte lema.

Lema 3.2.3. Para todo $k \geq 1$ e $t>1, \mathcal{L}_{\rho_{k}^{t}}\left(A_{k}^{t}\right)=k \cdot \log t$.

Demonstração. Considere-se a região circular $C_{j}^{t}, 1 \leq j<k$. Cada $z \in C_{j}^{t}$ pode-se escrever em coordenadas polares como $z=c_{j}+r \cdot e^{i \theta}$, com $\frac{a_{k}}{t^{k}}<r<a_{k}$ e $0 \leq \theta \leq \pi$. Observe-se que para 


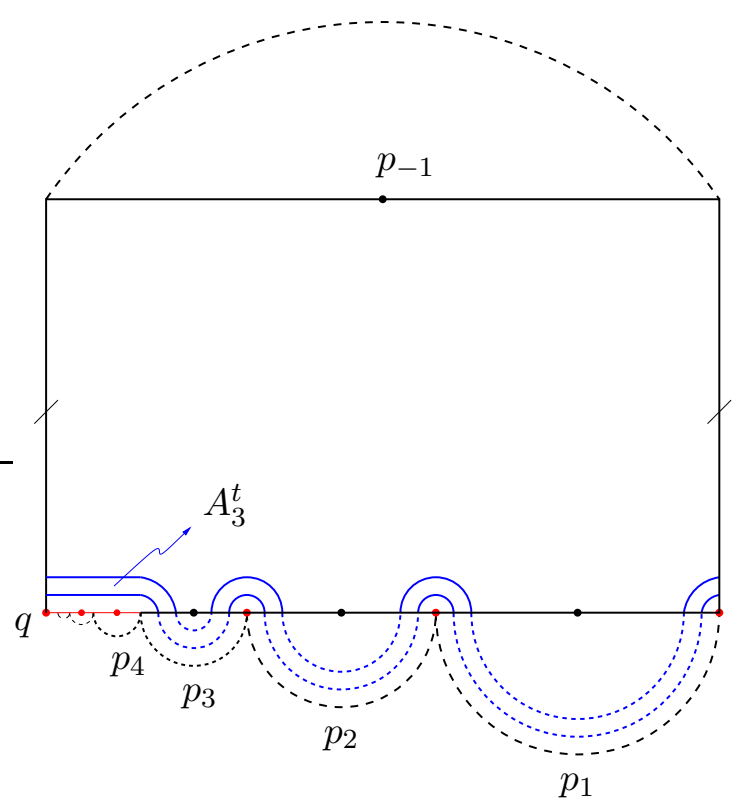

Figura 3.6: Anel $A_{3}^{t}$ em azul. Em vermelho mostra-se as componentes de $\pi_{\mathcal{P}}^{-1}\left(D_{3}\right)$

$z \in C_{j}^{t}, d\left(z, D_{k}\right)=\left|z-c_{j}\right|=r$. Fixando um ângulo $\theta_{0} \in[0, \pi]$, seja $\beta_{\theta}(s)=c_{j}+s \cdot e^{i \theta_{0}}, \frac{a_{k}}{t^{k}} \leq s \leq a_{k}$, isto é, o segmento que conecta as duas componentes de $\partial C_{i}^{t} \cap \partial \pi^{-1}\left(A_{k}^{t}\right)$, na semi-reta que parte de $c_{j}$ fazendo um ângulo $\theta_{0}$ com o eixo real, ver figura 3.7 à esquerda.
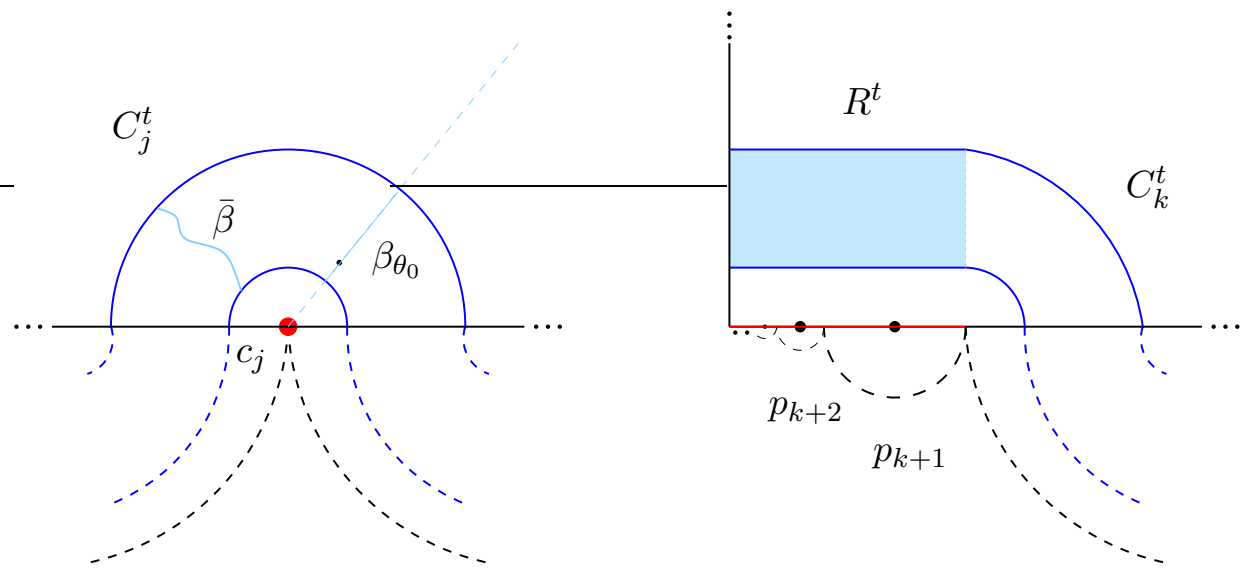

Figura 3.7: Região circular $C_{j}^{t}$ e região retangular $R^{t}$

Logo o $\rho_{k}^{t}$-comprimento de $\beta_{\theta_{0}}$ é

$$
\int_{\bar{\beta}} \rho_{k}^{t}(z)|d z|=\int_{\beta} \frac{|d z|}{d^{\mathcal{P}}\left(z, D_{k}\right)}=\int_{\frac{a_{k}}{t^{k}}}^{a_{k}} \frac{d s}{s}=\log \frac{a_{k}}{a_{k} / t^{k}}=k \cdot \log t
$$

Se $\bar{\beta}(s)=c_{j}+r(s) \cdot e^{i \theta(s)} \in C_{j}^{t}, s \in I$ é qualquer outro caminho que conecta as mesmas duas componenntes, assumiendo sem perda de generalidade que $r^{\prime}(s) \geq 0$, o $\rho_{k}^{t}$-comprimento de $\bar{\beta}(s)$ é,

$$
\int_{\beta} \rho_{k}^{t}(z)|d z|=\int_{I} \frac{\left|r^{\prime}(s)+i \cdot r(s) \cdot \theta^{\prime}(s)\right|}{r(s)} d s \geq \int_{I} \frac{r^{\prime}(s)}{r(s)} d s=k \cdot \log t
$$

Logo $\mathcal{L}_{\rho_{k}^{t}}\left(C_{j}^{t}\right)=k \cdot \log t$, para todo $1 \leq j \leq k-1$. Similarmente se prova que $\mathcal{L}_{\rho_{k}^{t}}\left(C_{0}^{t}\right)=\mathcal{L}_{\rho_{k}^{t}}\left(C_{k}^{t}\right)=$ $k \cdot \log t$. Em $R^{t}$, a métrica $\rho_{k}^{t}$ é a mesma métrica plana $\rho_{e}$, mas multiplicada por um fator $\frac{k \cdot \log t}{a_{k}\left(1-\frac{1}{t^{k}}\right)}$. Agora ficará claro por que a escolha desse fator: $\mathcal{L}_{\rho_{e}}\left(R^{t}\right)=a_{k}\left(1-\frac{1}{t^{k}}\right), \log \mathcal{L}_{\rho_{k}^{t}}\left(R^{t}\right)=k \cdot \log t$. A maneira como são colados as diferentes peças do anel mostra que $\mathcal{L}_{\rho_{k}^{t}}\left(A_{k}^{t}\right)=k \cdot \log t$. 
A $\rho_{k}^{t}$-área do anel $A_{k}^{t}$ é calculado no seguinte lema.

Lema 3.2.4. Para todo $k \geq 1$ et $>1, \mathcal{A}_{\rho_{k}^{t}}\left(A_{k}^{t}\right)=\frac{2 k^{2} \cdot \log ^{2} t}{1-\frac{1}{t^{k}}} \cdot \frac{\sum_{n>k} a_{n}}{a_{k}}+\pi \cdot k^{2} \cdot \log t$

Demonstração. A área euclideana de $R^{t}$ é $A_{\rho_{e}}\left(R^{t}\right)=2 \cdot a_{k} \cdot\left(1-\frac{1}{t^{k}}\right) \sum_{n>k} a_{n}$. Portanto,

$$
\begin{aligned}
\mathcal{A}_{\rho_{k}^{t}}\left(R^{t}\right) & =\frac{k^{2} \cdot \log ^{2} t}{a_{k}^{2}\left(1-\frac{1}{t^{k}}\right)^{2}} \cdot \mathcal{A}_{\rho_{e}}\left(R^{t}\right) \\
& =\frac{2 \cdot k^{2} \cdot \log ^{2} t}{1-\frac{1}{t^{k}}} \cdot \frac{\sum_{n>k} a_{n}}{a_{k}}
\end{aligned}
$$

Para $1 \leq j \leq k-1$, novamente escrevendo os pontos de $C_{j}^{t}$ como $z=c_{j}+r \cdot e^{i \cdot \theta}$ tem-se,

$$
\begin{aligned}
\mathcal{A}_{\rho_{k}^{t}}\left(C_{j}^{t}\right) & =\iint_{C_{j}^{t}} \frac{d x d y}{d^{\mathcal{P}}\left(z, D_{k}\right)^{2}} \\
& =\int_{0}^{\pi} \int_{\frac{a_{k}}{t^{k}}}^{a_{k}} \frac{1}{r^{2}} \cdot r d r d \theta \\
& =\int_{0}^{\pi} d \theta \cdot \int_{\frac{a_{k}}{t^{k}}}^{a_{k}} \frac{d r}{r} \\
& =\pi \cdot k \cdot \log t
\end{aligned}
$$

Similarmente $\mathcal{A}_{\rho_{k}^{t}}\left(C_{j}^{t}\right)=\frac{\pi}{2} \cdot k \cdot \log t$, para $j=0, k$. Assim,

$$
\begin{aligned}
\mathcal{A}_{\rho_{k}^{t}}\left(A_{k}^{t}\right) & =\mathcal{A}_{\rho_{k}^{t}}\left(R^{t}\right)+\sum_{j=0}^{k} \mathcal{A}_{\rho_{k}^{t}}\left(C_{j}^{t}\right) \\
& =\frac{k^{2} \cdot \log ^{2} t}{1-\frac{1}{t^{k}}} \cdot \frac{\sum_{n>k} a_{n}}{a_{k}}+\pi \cdot k^{2} \log t
\end{aligned}
$$

Feitos os cálculos da $\rho_{k}^{t}$-área e a $\rho_{k}^{t}$-largura do anel $A_{k}^{t}$, aplicando a desigualdade 3.1 do Lema 3.2.2, tem-se:

$$
\ell_{\infty}\left(\gamma_{k}\right) \leq \pi \cdot \frac{\mathcal{A}_{\rho_{k}^{t}}\left(A_{k}^{t}\right)}{\mathcal{L}_{\rho_{k}^{t}}\left(A_{k}^{t}\right)^{2}}=\pi\left(\frac{2 \sum_{n>k} a_{n}}{a_{k}} \cdot \frac{1}{1-\frac{1}{t^{k}}}+\frac{\pi}{\log t}\right)
$$

Como a desigualdade acima é valida para qualquer valor do parámetro $t>1$, fazendo agora $t \longrightarrow+\infty$, temos provado o seguinte resultado:

Teorema 3.2.5. No modelo base $S_{\infty}$ tem-se, para cada $k \geq 1$,

$$
\ell_{\infty}\left(\gamma_{k}\right) \leq 2 \pi \cdot \frac{1}{a_{k}} \cdot \sum_{n>k} a_{n}
$$

A melhora obtida na estimativa anterior com relação a densidade Euclideana é clara: tomando $r=a_{k}$ na estimativa com $\rho_{e}$, esta fica igual à $M_{k}=2 \pi \cdot \frac{1}{a_{k}} \cdot \sum_{n>k} a_{n}+\frac{\pi^{2}}{2} \cdot k$, onde o termo $\frac{\pi^{2}}{2} \cdot k$ 
corresponde a contribução dada pelas peças circulares do anel, enquanto que com a densidade $\rho_{k}^{t}$, tal contribuição fica anulada ao fazer $t \rightarrow+\infty$

Exemplo 3.2.6. Seja $0<\lambda<1$ e suponha que $a_{n} \asymp \lambda^{n}$, é dizer, existem $A_{0}, A_{1}>0$ tais que para todo $n \geq 1$,

$$
A_{0} \cdot \lambda^{n} \leq a_{n} \leq A_{1} \cdot \lambda^{n}
$$

Logo para cada $k \geq 1$,

$$
\ell_{\infty}\left(\gamma_{k}\right) \leq 2 \pi \cdot \frac{A_{1}}{A_{0}} \cdot \frac{\sum_{n>k} \lambda^{n}}{\lambda^{k}}=\frac{A_{1}}{A_{0}} \cdot \frac{2 \lambda \pi}{1-\lambda}
$$

Em particular se $a_{n}=\frac{1}{2^{n}}, n \geq 1$, então $\ell_{\infty}\left(\gamma_{k}\right) \leq 2 \pi$, para cada $k \geq 1$.

Exemplo 3.2.7. Suponha agora que $a_{n}=\frac{1}{n(n+1)}$, então $\sum_{n>k} a_{n}=\frac{1}{k}$, com o qual, para cada $k \geq 1$,

$$
\ell_{\infty}\left(\gamma_{k}\right) \leq 2 k \pi
$$

Neste exemplo não fica claro se $\left\{\ell_{\infty}\left(\gamma_{k}\right)\right\}_{k}$ sería limitado ou não.

Em vista dos dois exemplos anteriores, gostariamos de um criterio simple que permita determinar quando a coleção $\left\{\ell_{\infty}\left(\gamma_{k}\right) / k \geq 1\right\}$ está limitada superiormente. Uma versão do Lema de StolzCesàro fornecerá este criterio; ainda sua prova seja elementar, esta será feita a continuação.

Lema 3.2.8 (Stolz-Cesàro). Sejam $\left(a_{k}\right)_{k \geq 1},\left(b_{k}\right)_{k \geq 1}$ duas sequências de números reais positivos que convergem à 0 e suponha que $\left(a_{k}\right)_{k \geq 1}$ é estritamente decrescente.

$$
\text { Se } \limsup _{k \rightarrow+\infty} \frac{b_{k}-b_{k+1}}{a_{k}-a_{k+1}}=\lambda \text {, então } \limsup _{k \rightarrow+\infty} \frac{b_{k}}{a_{k}} \leq \lambda .
$$

Demonstração. Dado $\varepsilon>0$, considere-se $N \geq 1$ tal que para todo $k \geq N$,

$$
b_{k}-b_{k+1} \leq(\lambda+\varepsilon)\left(a_{k}-a_{k+1}\right)
$$

Logo para $n>k \geq N$ qualquer,

$$
b_{k}-b_{k+1}=\sum_{i=k}^{n}\left(b_{i}-b_{i+1}\right) \leq(\lambda+\varepsilon) \sum_{i=k}^{n}\left(a_{i}-a_{i+1}\right)=(\lambda+\varepsilon)\left(a_{k}-a_{k+1}\right)
$$

Fazendo $n \rightarrow+\infty$, tem-se $\frac{b_{k}}{a_{k}} \leq \lambda-\varepsilon$, para qualquer $k \geq N$.

Sendo $\varepsilon$ arbitrário, tem-se $\limsup _{k \rightarrow+\infty} \frac{b_{k}}{a_{k}} \leq \lambda$.

Proposição 3.2.9. Na esfera de papel furada $S_{\infty}$ do modelos base,

i) Se $\limsup _{k \rightarrow+\infty} \frac{a_{k+1}}{a_{k}}<1$, então $\sup _{k \geq 1} \ell_{\infty}\left(\gamma_{k}\right)<+\infty$.

ii) $S e \lim _{k \rightarrow+\infty} \frac{a_{k+1}}{a_{k}}=0$, ent $\tilde{a} o \lim _{k \rightarrow+\infty} \ell_{\infty}\left(\gamma_{k}\right)=0$. 
Demonstração. Considere-se $b_{k}:=\sum_{n>k} a_{n}, \operatorname{logo} b_{k} \rightarrow 0$ quando $k \rightarrow+\infty$. Lembre-se que a sequência $\left(a_{k}\right)_{k \geq 1}$ é decrescente. Como para todo $k \geq 1$

$$
\frac{b_{k}-b_{k+1}}{a_{k}-a_{k+1}}=\frac{a_{k+1}}{a_{k}-a_{k+1}}=\frac{\frac{a_{k+1}}{a_{k}}}{1-\frac{a_{k+1}}{a_{k}}},
$$

$\limsup _{k \rightarrow+\infty} \frac{a_{k+1}}{a_{k}}=\lambda<1$ implica que $\limsup _{k \rightarrow+\infty} \frac{b_{k}-b_{k+1}}{a_{k}-a_{k+1}} \leq \frac{\lambda}{1-\lambda}$, logo pelo Lema 3.2.8 e pela desigualdade 3.2 ,

$$
\limsup _{k \rightarrow+\infty} \ell_{\infty}\left(\gamma_{k}\right) \leq 2 \pi \limsup _{k \rightarrow+\infty} \frac{b_{k}}{a_{k}} \leq \frac{2 \pi \lambda}{1-\lambda}
$$

Assim, $\sup _{k \geq 1} \ell_{\infty}\left(\gamma_{k}\right)<+\infty$ e, no caso $\lambda=0, \lim _{k \rightarrow+\infty} \ell_{\infty}\left(\gamma_{k}\right)=0$.

Exemplo 3.2.10. Sejam $A_{0}, A_{1}>0$ tais que para todo $k \geq 1$,

$$
\frac{A_{0}}{k !} \leq a_{k} \leq \frac{A_{1}}{k !}
$$

Neste caso $\lim _{k \rightarrow+\infty} \frac{a_{k+1}}{a_{k}}=\lim _{k \rightarrow+\infty} \frac{A_{1}}{A_{0}} \cdot \frac{1}{k+1}=0, \log \lim _{k \rightarrow+\infty} \ell_{\infty}\left(\gamma_{k}\right)=0$.

Exemplo 3.2.11. Se a sequência $\left(a_{k}\right)_{k \geq 1}$ decresce à 0 polinomialmente, isto é, existem $A_{0}, A_{1}>0$ e $s>1$ tais que para todo $k \geq 1$,

$$
\frac{A_{0}}{k^{s}} \leq a_{k} \leq \frac{A_{1}}{k^{s}}
$$

então tem-se que,

$$
\begin{aligned}
\frac{1}{a_{k}} \cdot \sum_{n>k} a_{n} & \geq \frac{A_{0}}{A_{1}} \cdot \sum_{n>k}\left(\frac{k}{n}\right)^{s} \\
& =\frac{A_{0}}{A_{1}} \cdot \sum_{n \geq 1} \frac{1}{\left(1+\frac{n}{k}\right)^{s}} \\
& \geq \frac{A_{0}}{A_{1}} \cdot \sum_{n=1}^{k} \frac{1}{\left(1+\frac{n}{k}\right)^{s}} \\
& \geq \frac{A_{0}}{A_{1} \cdot 2^{s}} \cdot k
\end{aligned}
$$

Assim neste caso $\lim _{k \rightarrow+\infty} \frac{1}{a_{k}} \cdot \sum_{n>k} a_{n}=+\infty$, pelo qual o criterio 3.2 .9 não permite decidir se a família $\left\{\ell_{\infty}\left(\gamma_{k}\right) / k \geq 1\right\}$ está limitada superiormente. Cresceram os comprimentos das geodésicas $\gamma_{k}$ para infinito neste caso? Se a resposta for afirmativa, as curvas crescem no máximo linearmente; em efeito, para todo $k \geq 1$, 


$$
\begin{aligned}
\frac{1}{a_{k}} \cdot \sum_{n>k} a_{n} & \leq \frac{A_{1}}{A_{0}} \cdot \sum_{n \geq 1} \frac{1}{\left(1+\frac{n}{k}\right)^{s}} \\
& =\frac{A_{1}}{A_{0}} \cdot \sum_{i \geq 1} \sum_{j=1}^{k} \frac{1}{\left(i+\frac{j}{k}\right)^{s}} \\
& \leq \frac{A_{1}}{A_{0}} \cdot \sum_{i \geq 1} \frac{k}{\left(i+\frac{1}{k}\right)^{s}} \\
& \leq \frac{A_{1}}{A_{0}} \cdot k \cdot \sum_{i \geq 1} \frac{1}{i^{s}}
\end{aligned}
$$

$\operatorname{Logo} \limsup _{k \rightarrow+\infty} \frac{\ell_{\infty}\left(\gamma_{k}\right)}{k} \leq \frac{2 \pi \cdot A_{1}}{A_{0}} \cdot \zeta(s)$, sendo $\zeta(s):=\sum_{i \geq 1} \frac{1}{i^{s}}$

Fechamos esta seção com um corolario que permite determinar se o modelo Fuchsiano da flauta $S_{\infty}$ no modelo base é do primeiro tipo. Este é conseqüência imediata da Proposição 3.2.9 e da proposição 1.4.3.

Corolario 3.2.12. Se $\limsup _{k \rightarrow+\infty} \frac{a_{k+1}}{a_{k}}<1$, então o modelo Fuchsiano $\mathbf{G}_{\infty}$ de $S_{\infty}$ é um grupo Fuchsiano do primeiro tipo.

\subsection{Estimativas superiores das coordenadas de comprimento}

Nesta seção procura-se generalizar o Teorema 3.2.5 para o caso de uma esfera de papel furada qualquer associada à dobradura $(P, \mathcal{P})$. Suponha-se que $q^{1}, \ldots, q^{m}$ são os pontos irregulares de $S_{\mathcal{P}}$ e lembre-se que $\Gamma_{\infty}$ denota a multicurva que decompoe a $S_{\infty}$ em calças. Uma vez fixado um superíndice $j=1, \ldots, m$, precisa-se conseguir estimativas superiores para a família $\left\{\ell_{\infty}\left(\gamma_{k}^{j}\right)\right\}_{k \geq 0}$, pelo menos para todo $k$ suficientemente grande. A ideia novamente consiste em fazer úso do Lema 3.2.2 construindo para cada $k$ uma região annular $A_{j, k}^{t} \subset S_{\infty}$ redutível à $\gamma_{k}^{j}$ e uma densidade $\rho_{j, k}^{t} \in m_{A_{j, k}^{t}}$, sendo $t>1$ um parámetro real conveniente para optimizar a estimativa dada pelo lema. No modelo base, o anel $A_{k}^{t}=A_{1, k}^{t}$ foi definido como $A_{k}^{t}=B_{d^{\mathcal{P}}}\left(D_{k}, e_{k}\right) \backslash \bar{B}_{d^{\mathcal{P}}}\left(D_{k}, \frac{e_{k}}{t^{k}}\right)$, onde $e_{k}$ é a $d^{\mathcal{P}}$-distância entre os conjuntos $E_{k}$ e $D_{k}$; neste caso a estimativa superior de $\ell_{\infty}\left(\gamma_{k}\right)$ depende do quociente $\frac{m_{k}}{e_{k}}$, onde $m_{k}=m_{G_{\mathcal{P}}}\left(D_{k}\right)=\sum_{n>k} a_{n}$. Por tal razão é natural esperar no caso geral que, ao definir $A_{j, k}^{t}$ como o conjunto $B_{d^{\mathcal{P}}}\left(D_{k}^{j}, e_{k}^{j}\right) \backslash \bar{B}_{d^{\mathcal{P}}}\left(D_{k}^{j}, \frac{e_{k}^{j}}{t_{t_{k}^{j}}^{j}}\right)$, sendo $\#_{k}^{j}$ uma potencia adequada, e ao usar o Lema 3.2.2 $\operatorname{com} \rho_{j, k}^{t}(z)|d z|$ similar a densidade definida na seção anterior, pode-se conseguir uma estimativa da curva $\gamma_{k}^{j}$ proporcional ao quociente $\frac{m_{G_{\mathcal{P}}}\left(D_{k}^{j}\right)}{e_{k}^{j}}$, onde $e_{k}^{j}$ é definido por:

$$
e_{k}^{j}:=d_{\mathcal{P}}\left(D_{k}^{j}, E_{k}^{j}\right)
$$

Aquí é necessario ser cuidadoso com o argumento pois ao ser agora $P$ um multipolígono qualquer e ao estar $\pi_{\mathcal{P}}^{-1}\left(D_{k}^{j}\right)$ contido possívelmente em diferentes lados de $P$, o número $e_{k}^{j}$ tal vez não seja suficientemente pequenho para garantir que o conjunto $A_{j, k}^{t}$ como foi definido acima seja uma região annular, com o qual o argumento inteiro falha. Assim, na hora de construir o anel $A_{j, k}^{t}$, este dependerá de um raio $h_{k}^{j}$ a definir mais na frente o qual será menor ou igual que a distância $e_{k}^{j}$. Para simplificar a notação, denote-se por $m_{k}^{j}$ o valor: 


$$
m_{k}^{j}:=m_{G_{\mathcal{P}}}\left(D_{k}^{j}\right)
$$

Lembre-se que $m_{G_{\mathcal{P}}}(D):=m_{\partial P}\left(\pi_{\mathcal{P}}^{-1}(D)\right)$, para todo $D \subset G_{\mathcal{P}}$ Borel-mensurável, onde $m_{\partial P}$ denota a medida de Lebesgue 1-dimensional em $\partial P$.

Proposição 3.3.1. Para todo $j=1,2, \ldots, m$,

$$
\lim _{k \rightarrow+\infty} e_{k}^{j}=\lim _{k \rightarrow+\infty} m_{k}^{j}=0 .
$$

Demonstração. Como $\lim _{k \rightarrow+\infty} p_{k}^{j}=q^{j}$ e como,

$$
0 \leq e_{k}^{j}=d^{\mathcal{P}}\left(D_{k}^{j}, E_{k}^{j}\right) \leq d^{\mathcal{P}}\left(q^{j}, p_{k}^{j}\right),
$$

é claro que $\lim _{k \rightarrow+\infty} e_{k}^{j}=0$. Para ver que $\lim _{k \rightarrow+\infty} m_{k}^{j}=0$ basta provar que $\lim _{k \rightarrow+\infty} \operatorname{diam}_{G_{\mathcal{P}}} D_{k}^{j}=0$ pois, como $D_{k}^{j} \supset D_{k+1}^{j}, k \geq 1$, então $\bigcap_{k \geq 1} D_{k}^{j}=\left\{q^{j}\right\}$, com o qual,

$$
\lim _{k \rightarrow+\infty} m_{k}^{j}=\lim _{k \rightarrow+\infty} m_{G_{\mathcal{P}}}\left(D_{k}^{j}\right)=m_{G_{\mathcal{P}}}\left(\left\{q^{j}\right\}\right)=0
$$

Demostremos então o seguinte: Para todo $k \geq 1$ e $x \in D_{k}^{j}, d_{G_{\mathcal{P}}}\left(q^{j}, x\right) \leq \max _{n>k} d_{G_{\mathcal{P}}}\left(q^{j}, p_{n}^{j}\right)$. Seja $x_{0} \in D_{k}^{j}$ um ponto tal que $d_{G_{\mathcal{P}}}\left(q^{j}, x_{0}\right) \leq \max _{x \in D_{k}^{j}} d_{G_{\mathcal{P}}}\left(q^{j}, x\right)$ e $\delta:[0,1] \longrightarrow D_{k}^{j}$ um caminho com extremidades $\delta(0)=x_{0}$ e $\delta(1)=q^{j}$ que realiza esta distância. O Lema 2.3.4 implica que o ponto $x_{0}$ é uma extremidade do dendrito $D_{k}^{j}$, logo existe $t_{1} \in(0,1]$ tal que $\delta(t)$ é um ponto planar de $S_{\mathcal{P}}$ para $0<t<t_{1}$ e $\delta\left(t_{1}\right)$ é um ponto não-planar de $S_{\mathcal{P}}$, com o qual $\delta\left(\left[0, t_{1}\right)\right)$ é um aberto em $D_{k}^{j}$ que não o separa. Portanto $A+D_{k}^{j} \backslash \delta\left(\left[0, t_{1}\right)\right)$ é um subcontinuum proprio de $D_{k}^{j}$. Se $x_{0} \neq p_{n}^{j}$ para $n>k$, então $A$ contém $\left\{q^{j}\right\} \cup\left\{p_{k}^{j}\right\}_{n>k}$ o qual contradíz a definição do continuum $D_{k}^{j}$. Logo $x_{0}=p_{n}^{j}$ para algum $n>k$.

Como $m_{k}^{j} \rightarrow 0$ quando $k \rightarrow+\infty$, podemos tomar $K_{0}$ suficientemente grande de forma que para todo $k \geq K_{0}$, cada componente conexa de $\pi_{\mathcal{P}}^{-1}\left(D_{k}^{j}\right)$ seja um segmento ou um ponto contido no máximo em dois lados consecutivos do multipolígono $P$. Assim, se escrevemos à $\pi_{\mathcal{P}}^{-1}\left(D_{k}^{j}\right)$ como a união $\pi_{\mathcal{P}}^{-1}\left(D_{k}^{j}\right)=\bigsqcup_{i \in I} L_{i}$ de suas componentes conexas (Aquí será excluido da notação os índices $j, k$, abusando um pouco do idioma), cada componente $L_{i}$ é um segmento poligonal com extremidades $a_{i}^{-}$e $a_{i}^{+}$, sendo a primeira a extremidade esquerda e a segunda a direita,relativo à orientação do $\partial P$, e podemos clasificar cada uma destas componentes em cuatro tipos:

Tipo 1: $L_{i}$ é um ponto, com o qual $a_{i}^{-}=a_{i}^{+}=a_{i}$.

Tipo 2: $L_{i}$ é um segmento cujo interior está contido num lado só do multipolígono $P$.

Tipo 3: $L_{i}$ é um segmento cujo interior está contido em dois lados do multipolígono $P$ e contém um único vértice $v_{i}$ de $P$ com ângulo $\phi_{i}:=\xi_{P}\left(v_{i}\right)>\pi$.

Tipo 4: $L_{i}$ é um segmento cujo interior está contido em dois lados do multipolígono $P$ e contém um único vértice $v_{i}$ de $P$ com ângulo $\phi_{i}:=\xi_{P}\left(v_{i}\right)<\pi$.

Em consequência o conjunto de índices $I$ pode-se escrever como a união $I=I_{1} \sqcup I_{2} \sqcup I_{3} \sqcup I_{4}$ dependendo do tipo da componente $L_{i}$. Observe-se que no modelo base só existem componentes do 
tipo 1 e 2. No espaço de papel da figura 3.8, para $k=5$ tem-se $\pi_{\mathcal{P}}^{-1}\left(D_{5}^{1}\right)=\bigsqcup_{i=1}^{7} L_{i}$, onde $1,2,4,6 \in I_{1}$, $5 \in I_{2}, 3 \in I_{3}$ e $7 \in I_{4}$.

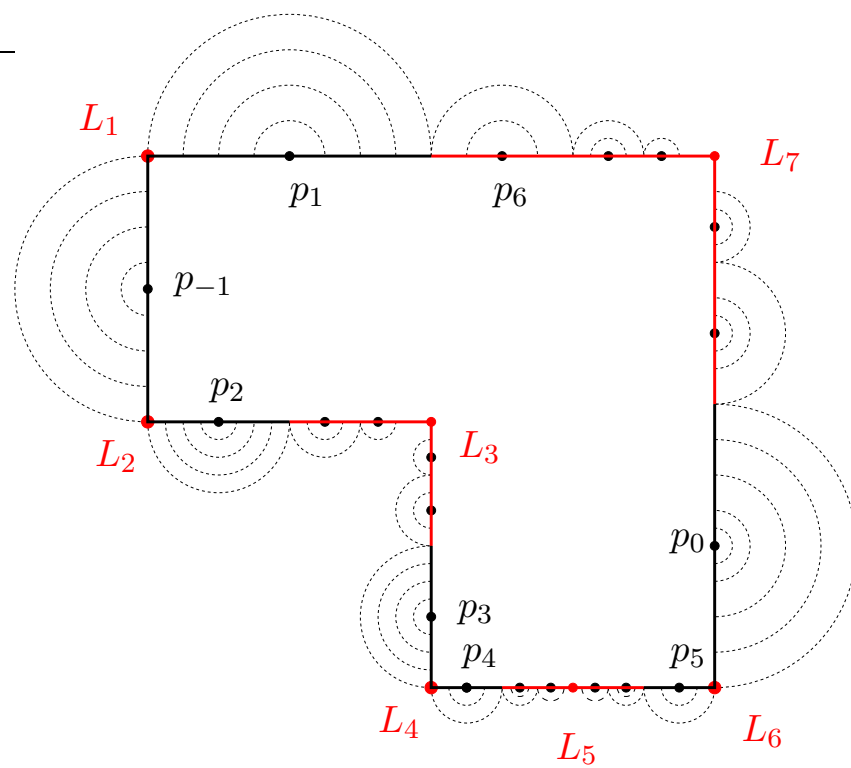

Figura 3.8: Em vermelho mostra-se as componentes de $\pi_{\mathcal{P}}^{-1}\left(D_{5}^{1}\right)$

Para construir o anel $A_{j, k}^{t}$ e posteriormente a densidade $\rho_{j, k}^{t}$, gostariamos ter uma boa descrição dos discos $B_{P}\left(L_{i}, h\right)$, para $h>0$ pequenho. Nos casos das componentes $L_{i}$ do tipo 1 e 2 e até do tipo 3 os discos $B_{P}\left(L_{i}, h\right)$ são relativamentes simples como mostra a figura 3.9: é fácil decompoer estos discos em peças retangulares e peças circulares. Quando $i \in I_{4}$, esta divisão do disco $B_{P}\left(L_{i}, h\right)$ não fica muito clara se o ângulo $\phi_{i}$ é pequenho, ver figura 3.10. Observe-se que esta diferenciação entre peças retangulares e circulares foi chave para a definição da densidade $\rho_{k}^{j}$ no modelo base.
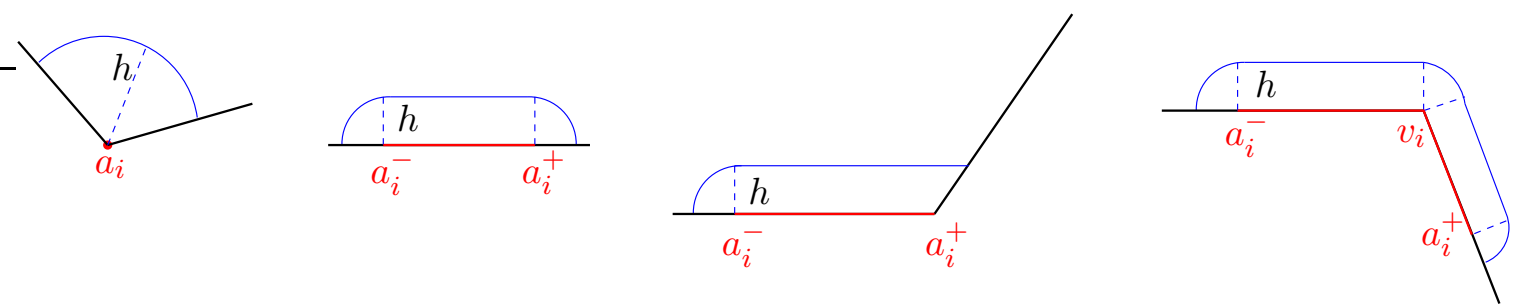

Figura 3.9: $O$ disco $B_{P}\left(L_{i}, h\right)$ com centro $L_{i}$ (em vermelho) do tipo 1, 2 e 3

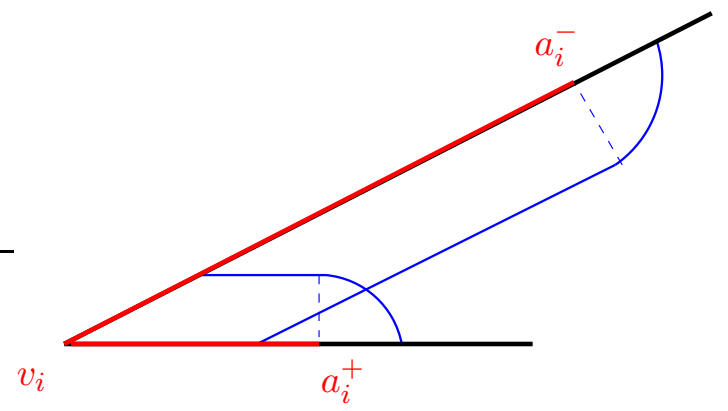

Figura 3.10: $B_{P}\left(L_{i}, h\right)$ para uma componente $L_{i}$ do tipo 4 e $h$ grande.

Para o caso geral também procuramos fazer esta divisão nos discos $B_{P}\left(L_{i}, h\right)$ em partes circulare 
e retangulares, indiferentemente do tipo da componente $L_{i}$, pelo qual, além dos valores $m_{k}^{j}$ e $e_{k}^{j}$ já definidos, usaremos o seguinte parámetro: Se $I_{4}=\emptyset$, defina-se $f_{k}^{j}:=\infty$ e se $I_{4} \neq \emptyset$, então,

$$
f_{k}^{j}:=\frac{1}{2} \cdot \min _{i \in I_{4}}\left\{d_{P}\left(v_{i}, a_{i}^{-}\right) \cdot \tan \left(\frac{\phi_{i}}{2}\right), d_{P}\left(v_{i}, a_{i}^{+}\right) \cdot \tan \left(\frac{\phi_{i}}{2}\right)\right\}
$$

Por último, defina-se:

$$
h_{k}^{j}:=\min \left\{e_{k}^{j}, f_{k}^{j}\right\}
$$

Uma vez definidos todos estos parámetros podemos enunciar o resultado principal desta seção.

Teorema 3.3.2 (Criterio para as estimativas de $\ell_{\infty}\left(\gamma_{k}^{j}\right)$ ). Considere-se uma esfera de papel furada $S_{\infty}$ associada à esfera de papel $S_{\mathcal{P}}$ com $m$ pontos irregulares. Então existe $K_{0} \geq 1$ tal que para todo $1 \leq j \leq m$ e $k \geq K_{0}$,

$$
\ell_{\infty}\left(\gamma_{k}^{j}\right) \leq \pi \cdot \frac{m_{k}^{j}}{h_{k}^{j}}
$$

A prova deste resultado, aínda o argumentos seja o mesmo usado no modelo base, precisa de alguns lemas técnicos, pelo qual será desenvolvida na próxima seção. Por enquanto mostraremos no que resta da seção alguns exemplos onde é aplicado o criterio acima.

Exemplo 3.3.3. Seja $P$ um triângulo isosceles cujos vértices são $v, x_{1}$ e $x_{2}$, sendo o lado com extremidades $x_{1}$ e $x_{2}$ a base do triângulo e os lados $\left[v, x_{1}\right]$ e $\left[v, x_{2}\right]$ os lados de igual comprimento. Suponha que o ângulo no vértice $v$ é $\phi \in(0, \pi)$. Considere-se $\mathcal{P}=\left\{\left\langle\alpha_{n}, \alpha_{n}^{\prime}\right\rangle\right\}_{n \geq-1}$ a dobradura de papel mostrada na figura 3.11, onde $\left|\left\langle\alpha_{2 n-1}, \alpha_{2 n-1}^{\prime}\right\rangle\right|=\left|\left\langle\alpha_{2 n}, \alpha_{2 n}^{\prime}\right\rangle\right|=\frac{1}{2^{n}}$, para cada $n \geq 1$.

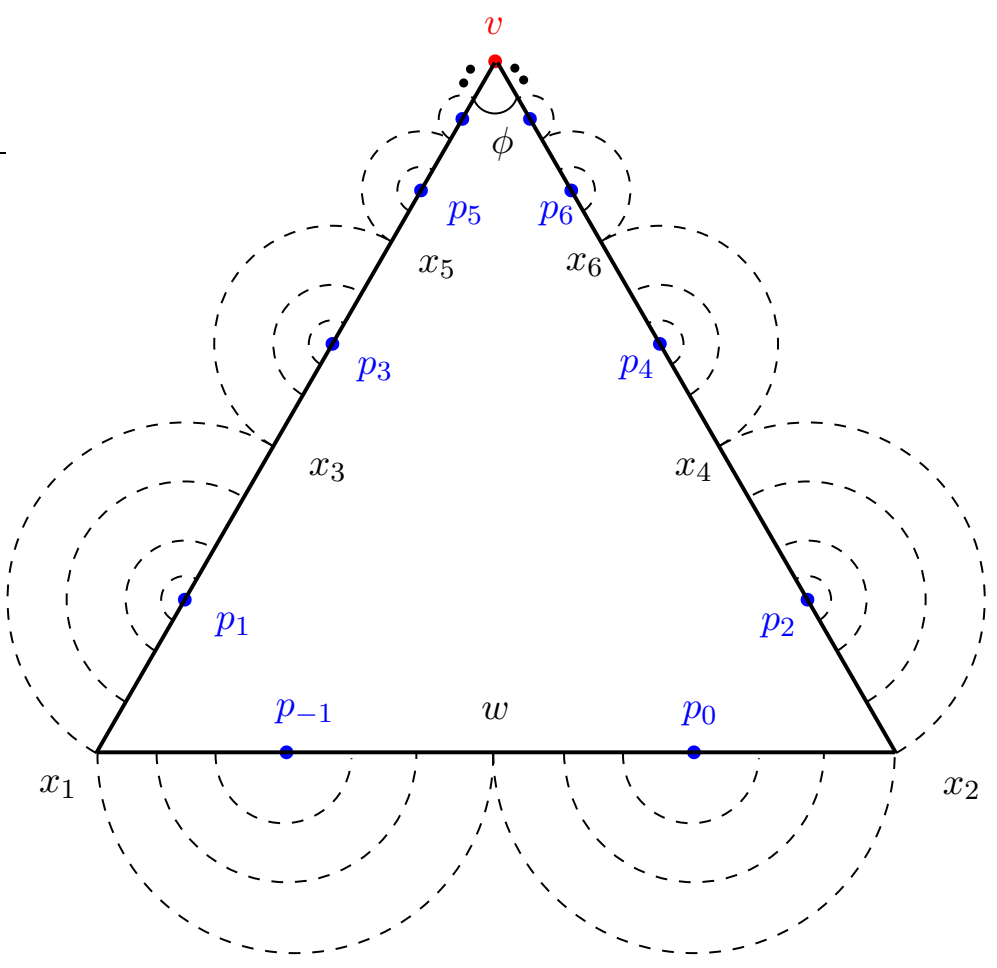

Figura 3.11: Esfera de papel do exemplo 3.3.3

Aquí os pares $\alpha_{n} \cup \alpha_{n}^{\prime}$ estão contidos no lado $\left[v, x_{1}\right]$ quando $n$ seja impar e no lado $\left[v, x_{2}\right]$ quando $n$ seja par. O espaço de papel $S_{\mathcal{P}}$ tem uma única irregularidade $q=\pi_{\mathcal{P}}(v)$. Para cada $k \geq 1$, observe-se que, 


$$
\begin{gathered}
\pi_{\mathcal{P}}^{-1}\left(D_{2 k-1}\right)=\left[v, x_{2 k+1}\right] \cup\left[v, x_{2 k}\right] \cup\left\{w, x_{1}, x_{2}, \ldots, x_{2 k-1}\right\}, \\
\pi_{\mathcal{P}}^{-1}\left(D_{2 k}\right)=\left[v, x_{2 k+1}\right] \cup\left[v, x_{2 k+2}\right] \cup\left\{w, x_{1}, x_{2}, \ldots, x_{2 k}\right\},
\end{gathered}
$$

Pelo qual, $m_{2 k-1}=4 \sum_{n>k} \frac{1}{2^{n}}+\frac{2}{2^{k}}=\frac{6}{2^{k}}$ e $m_{2 k}=4 \sum_{n>k} \frac{1}{2^{n}}=\frac{4}{2^{k}}$. Tem-se também que $e_{2 k-1}=$ $e_{2 k}=d^{\mathcal{P}}\left(p_{2 k-1}, q\right)=\frac{1}{2^{k}}$ e $f_{2 k-1}=f_{2 k}=\frac{1}{2} d^{\mathcal{P}}\left(v, x_{2 k+1}\right) \cdot \tan \frac{\phi}{2}=\frac{1}{2^{k}} \tan \frac{\phi}{2}$. Assim $h_{k}=e_{k}$ se, e somente se, $\phi \geq \frac{\pi}{2}$. Neste caso, aplicando o Teorema 3.3.2 tem-se,

$$
\ell_{\infty}\left(\gamma_{2 k-1}\right) \leq 6 \pi \text { e } \ell_{\infty}\left(\gamma_{2 k}\right) \leq 4 \pi
$$

Se em cambio $\phi<\frac{\pi}{2}$, então para todo $k$ suficientemente grande,

$$
\ell_{\infty}\left(\gamma_{2 k-1}\right) \leq \frac{6 \pi}{\tan \frac{\phi}{2}} \text { e } \ell_{\infty}\left(\gamma_{2 k}\right) \leq \frac{4 \pi}{\tan \frac{\phi}{2}}
$$

Exemplo 3.3.4. Considere-se $P$ o quadrado unitario e $\mathcal{P}=\bigcup_{j=1,2,3,4}\left(\left\{\left\langle\alpha_{k}^{j}, \alpha_{k}^{j^{\prime}}\right\rangle\right\}_{k \geq 1} \cup\left\{\left\langle\beta_{k}^{j}, \beta_{k}^{j^{\prime}}\right\rangle\right\}_{k \geq 0}\right)$ o pareamento mostrado na figura 3.12, onde cada superíndice $j$ corresponde com uma subfamília de pareamentos que cobrem cada lado e estos são os mesmos a menos de uma rotação. Os subsegmentos $\alpha_{k}^{j}$ e $\alpha_{k}^{j^{\prime}}$ produzem um ponto de dobradura $p_{k}^{j}$ e o segmento $\alpha_{k}^{j} \cup \alpha_{k}^{j^{\prime}}$ ubica-se entre os subsegmentos $\beta_{k-1}^{j}$ e $\beta_{k}^{j}$, ficando o primeiro na esquerda e o segundo na direita (relativo à orientação do $\partial P$ ); a sua vez, o subsegmento $\beta_{k-1}^{j^{\prime}}$ ubica-se na direita do subsegmento $\beta_{k}^{j^{\prime}}$. Sejam $a, b \in(0,1)$ tais que para cada $k \geq 1$ e $j=1,2,3,4,\left|\left\langle\alpha_{k}^{j}, \alpha_{k}^{j^{\prime}}\right\rangle\right|=a^{k}$ e $\left|\left\langle\beta_{k}^{j}, \beta_{k}^{j^{\prime}}\right\rangle\right|=b^{k}$. Observe-se que $S_{\mathcal{P}}$ tem quatro pontos irregulares $q^{j}, j=1,2,3,4$, um em cada lado do quadrado, os quais são pontos de acumulação de pontos 1-cônicos e 3-cônicos. Logo a esfera furada $S_{\infty}$ corresponde com uma gaita de fole justa de ordem 4.

Fixe um superíndice $j$ e observe que para todo $k \geq 0, \pi_{\mathcal{P}}^{-1}\left(D_{k}^{j}\right)$ consiste num único segmento contido no lado $j$, logo é do tipo 2. Assim tem-se $h_{k}^{j}=e_{k}^{j}=b^{k}$. Por outro lado é fácil ver que $m_{k}^{j}=2 \sum_{n>k} b^{k}+2 \sum_{n>k} a^{k}$. Portanto,

$$
\ell_{\infty}\left(\gamma_{k}^{j}\right) \leq 2 \pi\left[\frac{b}{1-b}+\left(\frac{a}{b}\right)^{k} \frac{a}{1-a}\right]
$$

Observe-se que se $a \leq b$, então todas as curvas tem comprimento limitado por acima.

O seguinte resultado é a generalização do Corolario 3.2.12 no modelo base para o caso geral de uma esfera de papel furada qualquer.

Proposição 3.3.5. Seja $S_{\mathcal{P}}$ uma esfera de papel cujo conjunto de pontos irregulares $\mathcal{Q}$ é finito. Com a notação da seção 3.1 e desta seção, suponha que para todo $1 \leq j \leq m$,

$$
\limsup _{k \rightarrow+\infty} \frac{m_{k}^{j}}{h_{k}^{j}}<+\infty
$$

Então o modelo Fuchsiano $\mathbf{G}_{\infty}$ da esfera furada $S_{\infty}$ é do primeiro tipo. Além disso, a estrutura complexa de $S_{\mathcal{P}} \backslash \mathcal{Q}$ extende-se a toda a esfera $S_{\mathcal{P}}$. 


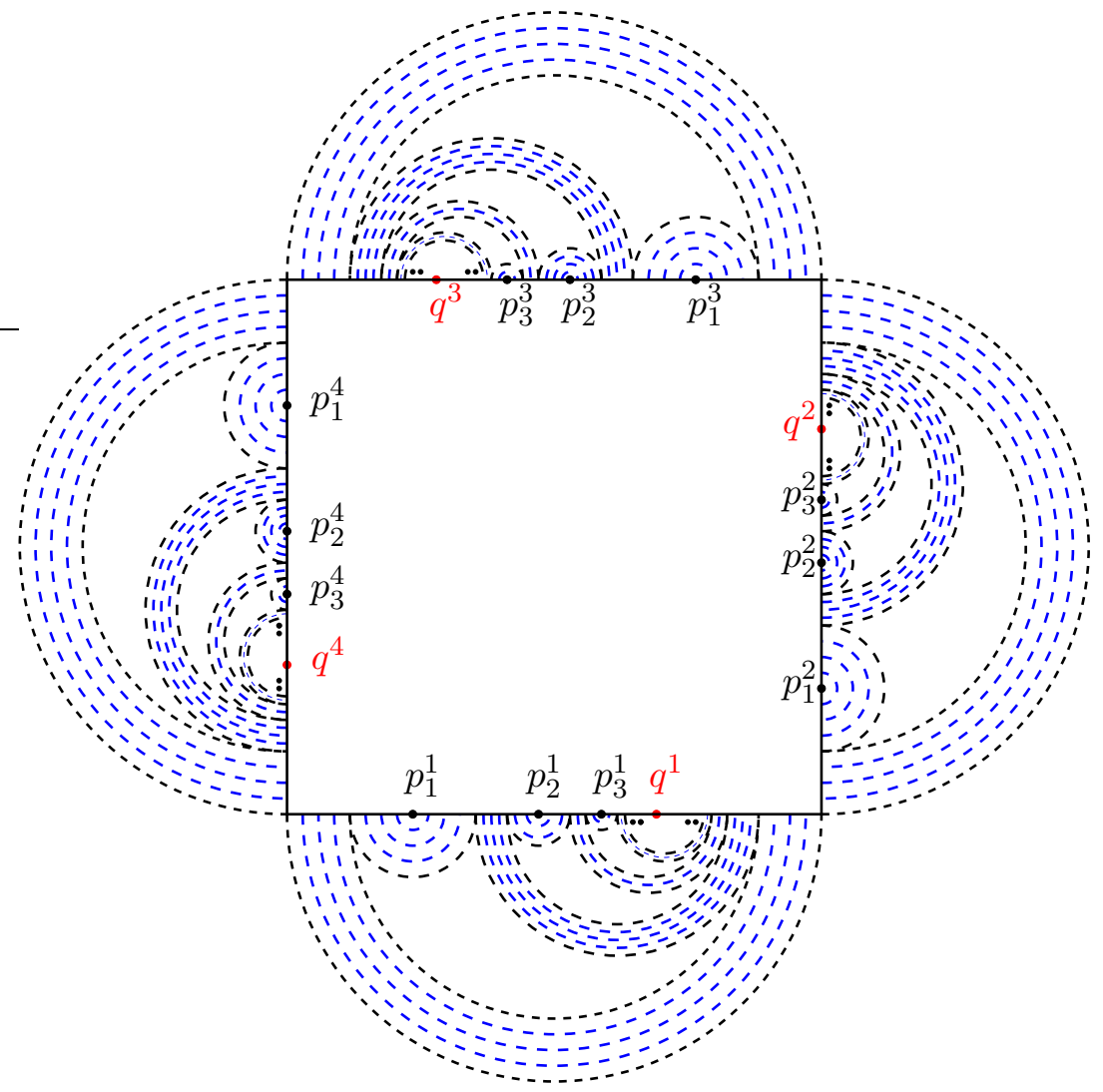

Figura 3.12: Esfera de papel do exemplo 3.3.4

Demonstração. Tome $M=\max _{1 \leq j \leq m} \sup _{k \geq 0} \ell_{\infty}\left(\gamma_{k}^{j}\right)$. Por hipótese, o Teorema 3.3 .2 implica que $M<+\infty$. Como $\eta\left(\ell_{\infty}\left(\gamma_{k}^{j}\right)\right) \geq \eta(M)>0$, para todo $k \geq 0$ e $1 \leq j \leq m$, então para cada $j=1, \ldots m$,

$$
\sum_{k=0}^{+\infty} \eta\left(\ell_{\infty}\left(\gamma_{k}^{j}\right)\right)=+\infty
$$

A Proposição 1.4.6 implica que $G_{\infty}$ é um grupo Fuchsiano do primeiro tipo. Para provar que a estrutura complexa extende-se ao ponto irregular $q^{j}$, considere-se cada colar $\mathbb{A}_{k}=\mathbb{A}_{\gamma_{k}^{j}}, k \geq 1$. Pelo Lema do colar, esta coleção é duas a duas disjunta, de fato esta coleção consiste numa família concentrica de aneis com respeito ao ponto $q^{j}$. Sendo $\ell_{\infty}\left(\gamma_{k}^{j}\right) \leq M$, então existe uma constante $c>0$ tal que $\operatorname{Mod} \mathbb{A}_{k} \geq c$ para todo $k \geq 1$. Logo $\sum_{k \geq 1} \operatorname{Mod} \mathbb{A}_{k}=\infty$ o que implica que em $S_{\mathcal{P}} \backslash \mathcal{Q}$, o fin $q^{j}$ corresponde a um furo, logo pelo Teorema da singularidade removível, a estrutura complexa pode-se extender a este ponto.

A seguinte proposição dá uma condição suficiente para que as distâncias $d_{\mathcal{T}}$ e $d_{\mathcal{L}}$ não sejam topológicamente equivalentes em $\mathcal{T}\left(S_{\infty}\right)$.

Corolario 3.3.6. Se existe $1 \leq j \leq m$ tal que,

$$
\liminf _{k \rightarrow+\infty} \frac{m_{k}^{j}}{e_{k}^{j}}=0,
$$

então, no espaço de Teichmüller $\mathcal{T}\left(S_{\infty}\right)$, a métricas de Teichmüller $d_{\mathcal{T}}$ e a métrica do comprimento espectral $d_{\mathcal{L}}$ não são topológicamente equivalentes. 
Demonstração. O Teorema 3.3.2 implica que existe uma subsequência de geodésica $\left(\gamma_{k_{n}}\right)_{n=1}^{+\infty}$ tal que $\ell_{\infty}\left(\gamma_{k_{n}}\right) \searrow 0$ quando $n \rightarrow+\infty$, logo a Proposição 1.3 .13 implica que $d_{\mathcal{T}}$ e $d_{\mathcal{L}}$ geram diferentes topologías em $\mathcal{T}\left(S_{\infty}\right)$.

\subsection{Prova do Teorema 3.3.2}

Suponha em principio que a esfera de papel $S_{\mathcal{P}}$ é obtido como quociente métrico de um único polígono $P$. Suponha também que foi fixado um superíndice $j=1, \ldots, m$ correspondente ao ponto irregular $q^{j}$, logo no desenvolvimento da prova do Teorema 3.3.2 será surpimido tal superíndice para aliviar a notação. Lembre-se que existe $K_{0} \geq 1$ tal que para $k \geq K_{0}$, as componentes $L_{i}$ de $\pi_{\mathcal{P}}^{-1}\left(D_{k}\right)$ são tipo 1, 2, 3 ou 4. O primeiro objetivo será mostrar que tais componentes estão suficientemente espalhados na fronteira de $P$. Para conseguir isso olhemos primeiro as extremidades $a_{i}^{-}$e $a_{i}^{+}$de cada $L_{i}$.

Lema 3.4.1. Para todo $i \in I, \pi_{\mathcal{P}}\left(a_{i}^{ \pm}\right) \in \mathcal{V} \cup\{q\}$, mas não são pontos 1-cônicos.

Demonstração. Dado $i \in I$ queremos ver que $\pi_{\mathcal{P}}\left(a_{i}^{-}\right) \in \mathcal{V} \cup\{q\}$. Suponha por absurdo que $\pi_{\mathcal{P}}\left(a_{i}^{-}\right)$é um ponto planar, logo existe $\left\langle\alpha, \alpha^{\prime}\right\rangle \in \mathcal{P}$ tal que $a_{i}^{-} \in$ int $\alpha$. Tome-se $\varepsilon>0$ suficientemente pequenho de forma que $\left(a_{i}^{-}-\varepsilon, a_{i}^{-}\right) \not \subset D_{k}$. Pelo Lema 2.4.3 o caminho simple $\delta \subset D_{k}$ que conecta $q$ com $\pi_{\mathcal{P}}\left(a_{i}^{-}\right)$extende-se a um caminho simple $\bar{\delta} \subset D_{k}$ com ponto final uma extremidade do dendrito $D_{k}$, o que resulta numa contradição pois $\bar{\delta}$ deve conter à $\pi_{\mathcal{P}}\left(\left(a_{i}^{-}-\varepsilon, a_{i}^{-}\right)\right)$. Portanto $\pi_{\mathcal{P}}\left(a_{i}^{-}\right) \in \mathcal{V} \cup\{q\}$.

Usando novamente redução ao absurdo, suponha que $\pi_{\mathcal{P}}\left(a_{i}^{-}\right)$é um ponto 1-cônico. Seja $\left\langle\alpha, \alpha^{\prime}\right\rangle \in$ $\mathcal{P}$ tal que $\pi_{\mathcal{P}}\left(a_{i}^{-}\right)$é o ponto de dobradura deste pareamento; suponha que $\alpha$ está na esquerda $\alpha^{\prime}$. Se $i \notin I_{1}$, pode-se tomar $\varepsilon>0$ suficientemente pequenho tal que $\left[a_{i}^{-}, a_{i}^{-}+\varepsilon\right) \subset L_{i} \cap \alpha^{\prime}$ e $\left(a_{i}^{-}-\varepsilon, a_{i}^{-}\right] \subset \alpha$. Claramente $\pi_{\mathcal{P}}\left(\left(a_{i}^{-}-\varepsilon, a_{i}^{-}\right]\right)=\pi_{\mathcal{P}}\left(\left[a_{i}^{-}, a_{i}^{-}+\varepsilon\right)\right)$, porém $\pi_{\mathcal{P}}\left(\left(a_{i}^{-}-\varepsilon, a_{i}^{-}\right]\right) \not \subset D_{k}$ pela definição da extremidade esquerda $a_{i}^{-}$enquanto que $\pi_{\mathcal{P}}\left(\left[a_{i}^{-}, a_{i}^{-}+\varepsilon\right)\right) \subset D_{k}$, com o qual temos uma contradição. Se $i \in I_{1}$ tem-se $a_{i}^{-}=a_{i}^{+}$. Um argumento similar ao usado para demostrar que $\pi_{\mathcal{P}}\left(a_{i}^{-}\right)$não é planar leva-nos também a uma contradição. Conclui-se assim que $\pi_{\mathcal{P}}\left(a_{i}^{-}\right)$não é um ponto 1-cônico. A demostração para a extremidade $a_{i}^{+}$é análoga.

Lema 3.4.2. Sejam $i_{1}, i_{2}, \ldots, i_{n} \in I$ tais que $\pi_{\mathcal{P}}^{-1}(q) \cap\left(L_{i_{1}} \cup L_{i_{2}} \cup \ldots \cup L_{i_{n}}\right)=\emptyset$, então existem $i_{n+1} \in I, i_{n+1} \neq i_{1}, \ldots, i_{n}, 1 \leq l \leq n, z \in L_{i_{n+1}}$ e $z^{\prime} \in L_{i_{l}}$, tais que $z \mathcal{P} z^{\prime}$.

Demonstração. Observe-se que $A=\pi_{\mathcal{P}}\left(L_{i_{1}} \cup \ldots L_{i_{n}}\right) \subsetneq D_{k}$ é fechado e não vazío em $D_{k}$. Como $\pi_{\mathcal{P}}^{-1}(q) \cap L_{i_{l}}$ para todo $1 \leq l \leq n$, pelo Lema 3.4.1 não podem existir componentes $L_{i}$, com $i \neq i_{l}$, à uma $d_{\partial P}$-distância arbitrariamente pequenha de $L_{i_{l}}$. Em consequência existe $\varepsilon>0$ tal que $\left(a_{i_{l}}^{-}-\varepsilon, a_{i_{l}}^{+}+\varepsilon\right) \cap L_{i}=\emptyset$ para todo $i \neq i_{1}, \ldots, i_{n}$ e $l=1, \ldots, n$.

Lembre-se que $d_{\mid G_{\mathcal{P}}}^{\mathcal{P}}=\left(d_{\partial P}\right)^{\mathcal{P}}$, ver Proposição $\left.2.2 .5 i i\right)$. Como $A$ não pode ser aberto em $D_{k}$ pois por definição $D_{k}$ é conexo, devem existir $i_{n+1} \neq i_{1}, \ldots, i_{n}, z \in L_{i_{n+1}}$ e $z^{\prime} \in L_{i_{1}} \cup \ldots \cup L_{i_{n}}$ tais que $d^{\mathcal{P}}\left(z, z^{\prime}\right)=0$ e isto só vai a acontecer se $z \mathcal{P} z^{\prime}$.

Sejam $L_{i_{1}}$ e $L_{i_{2}}$ dois componentes consecutivos de $\pi_{\mathcal{P}}^{-1}\left(D_{k}\right)$, é dizer, tais que no intervalo $\left(a_{i_{1}}^{+}, a_{i_{2}}^{-}\right)$ não existe outra componente de $\pi_{\mathcal{P}}^{-1}\left(D_{k}\right)$. Dize-se que o intervalo $\left(a_{i_{1}}^{+}, a_{i_{2}}^{-}\right)$é uma brecha do $D_{k}$ se existe $\left\langle\alpha, \alpha^{\prime}\right\rangle \in \mathcal{P}$ tal que int $\alpha=\left(a_{i_{1}}^{+}, a_{i_{2}}^{-}\right)$. Um exemplo de brecha do $D_{k}$ para todo $k \geq 6$ é mostrado na figura 3.13; observe-se que neste exemplo $P$ consiste numa união disjunta de dois polígonos e não é um polígo no só como estamos assumindo até agora.

Lema 3.4.3. Se $P$ é um polígono, não existem brechas do $D_{k}$ para todo $k \geq K_{0}$. 


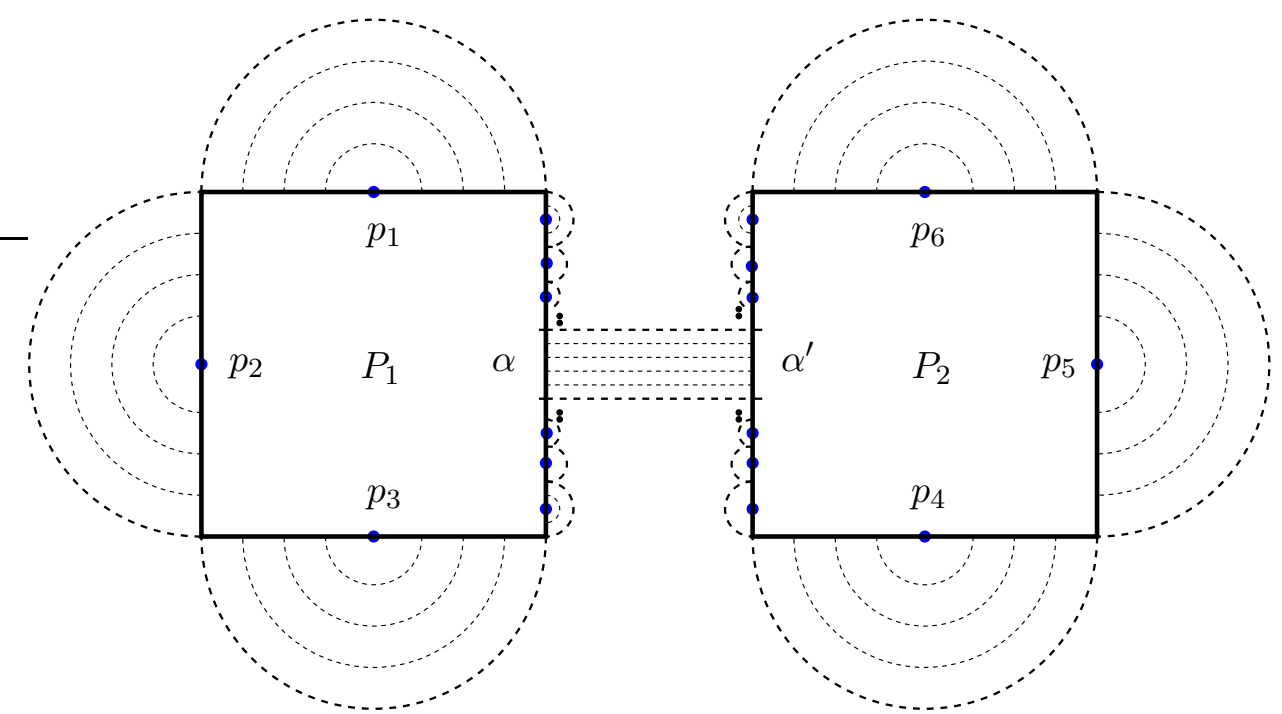

Figura 3.13: Desenho de uma brecha de $D_{k}$, para todo $k \geq 1$. As extremidades dos intevalos $\alpha$ e $\alpha^{\prime}$ são pre-imagems da irregularidade do espaço de papel $S_{\mathcal{P}}$.

Demonstração. Suponha por redução ao absurdo que existe algum $k$ e um pareamento $\left\langle\alpha, \alpha^{\prime}\right\rangle \in \mathcal{P}$ tal que int $\alpha$ é uma brecha de $D_{k}$. Observe-se que $\partial P \backslash\left(\right.$ int $\alpha \cup$ int $\left.\alpha^{\prime}\right)$ é a união disjunta de dois arcos fechados $T_{1}$ e $T_{2}$, cada um dos quais contém pelo menos uma componente de $\pi_{\mathcal{P}}^{-1}\left(D_{k}\right)$.

Lembre-se que a relação $\mathcal{P}$ em $\partial P$ é desvinculada, ver Teorema 2.3.1. Em consequência a relação $\sim_{\mathcal{P}}$ também é desvinculada em $\partial P$, ver Lema 2.3.3, pelo qual, ou $\pi_{\mathcal{P}}^{-1}(q) \cap T_{1}=\emptyset$ ou $\pi_{\mathcal{P}}^{-1}(q) \cap T_{2}=\emptyset$. Suponha que $\pi_{\mathcal{P}}^{-1}(q) \cap T_{1}=\emptyset$ e $\pi_{\mathcal{P}}^{-1}(q) \cap T_{2} \neq \emptyset$.

Seja $L_{i_{1}} \subset T_{1}$ uma componente de $\pi_{\mathcal{P}}^{-1}\left(D_{k}\right)$. Pelo Lema 3.4 .2 existem $i_{1} \neq i_{2}, z_{1} \in L_{i_{1}}$ e $z^{\prime} \in L_{i_{2}}$ tais que $z_{1} \mathcal{P} z_{1}^{\prime}$. Sendo $\mathcal{P}$ desvinculada em $\partial P$ tem-se que $L_{i_{2}} \subset T_{1}$. Usando novamente o Lema 3.4.2 e o fato de $\mathcal{P}$ ser desvinculada em $\partial P$ obtem-se $i_{3} \neq i_{1}, i_{2}$ tal que $L_{i_{3}} \subset T_{1}$. Repetindo o argumento infinitas vezes construye-se uma sequência de componentes diferentes $\left(L_{i_{n}}\right)_{n \geq 1}$ de $\pi_{\mathcal{P}}^{-1}\left(D_{k}\right)$, todas contidas em $T_{1}$. Considerando que cada $L_{i_{n}}$ contém pelo menos uma $\pi_{\mathcal{P}}$-preimagem de um ponto cônico (Lema 3.4.1), existe $z_{1} \in T_{1}$ ponto de acumulação de $\pi_{\mathcal{P}}^{-1}\left(\mathcal{V} \cap D_{k}\right)$. Isto implica que $\pi_{\mathcal{P}}\left(z_{1}\right)=q$ o qual é uma contradição.

Lema 3.4.4. Para todo $k \geq K_{0}$ e $i_{1}, i_{2} \in I(k)=I, i_{1} \neq i_{2}$,

$$
d_{\partial P}\left(L_{i_{1}}, L_{i_{2}}\right) \geq 2 e_{k}
$$

Em consequência o número de componentes conexas de $\pi_{\mathcal{P}}^{-1}\left(D_{k}\right)$ é finito para cada $k \geq K_{0}$.

Demonstração. Basta provar que dadas duas componentes consecutivas $L_{i_{1}}$ e $L_{i_{2}}$ de $\pi_{\mathcal{P}}^{-1}\left(D_{k}\right)$, sendo $L_{i_{1}}$ à esquerda de $L_{i_{2}}$, então $d_{\partial P}\left(a_{i_{1}}^{+}, a_{i_{2}}^{-}\right) \geq 2 e_{k}$. Pelo Lema 3.4 .3 (Lembre-se que estamos assumindo que $P$ é um polígono) o intervalo $\left(a_{i_{1}}^{+}, a_{i_{2}}^{-}\right)$não é uma brecha, logo existe $w \in\left(a_{i_{1}}^{+}, a_{i_{2}}^{-}\right)$ tal que $\pi_{\mathcal{P}}(w) \in \overline{\mathcal{V}}$. Como $L_{i_{1}}$ e $L_{i_{2}}$ são consecutivos, então $\pi_{\mathcal{P}}(w) \notin D_{k}, \operatorname{logo} \pi_{\mathcal{P}}(w) \in E_{k}$. Assim,

$$
d_{\partial P}\left(a_{i_{1}}^{+}, a_{i_{2}}^{-}\right)=d_{\partial P}\left(a_{i_{1}}^{+}, w\right)+d_{\partial P}\left(w, a_{i_{2}}^{-}\right) \geq 2 e_{k}
$$

Dado que $e_{k}>0$ e $|\partial P|$ é finito, não podem existir infinitas componentes $L_{i}$ de $\pi_{\mathcal{P}}^{-1}\left(D_{k}\right)$.

Para cada $k \geq 1$ denote-se por $\#_{k}$ ao número de componentes de $\pi_{\mathcal{P}}^{-1}\left(D_{k}\right)$. No modelo base e no exemplo 3.3 .3 a sequência $\left(\#_{k}\right)_{k}$ cresce linearmente com relação à $k$ : $\#_{k}=k+1$ no caso do modelo base e $\#_{k}=k+3$ no exemplo 3.3.3. No exemplo 3.3.4 em cambio, $\#_{k}^{j}$ é constante igual à 1 , para $j=1,2,3,4$. Fixando para todo $k \geq K_{0}$ um índice $i_{1} \in I$ (a verdade a notação certa deveria 
ser $I(j, k)$ ou $I(k)$, mas continuaremos suprimindo o superíndice $j$ nesta seção e do índice $k$ para o conjunto $I$ ), podemos ordenar os elementos de $I$ como $i_{1}, i_{2}, \ldots, i_{\#_{k}}$, de forma que $L_{i_{l}}$ está na esquerda de $L_{i_{l+1}}$ e são consecutivos, para $1 \leq l \leq \#_{k}$, tomando $i_{\#_{k}+1}=i_{1}$.

Lema 3.4.5. Para todo $k \geq K_{0}$ e $1 \leq l \leq \#_{k}, a_{i_{l}}^{+} \sim_{\mathcal{P}} a_{i_{l+1}}^{-}$.

Demonstração. Pelo Lema 3.4.4, a esquerda e a direita de cada componente $L_{i}, i \in I$, somente há $\pi_{\mathcal{P}}$-preimagems de pontos planares de $S_{\mathcal{P}}$ numa vizinhança de $L_{i}$. Sendo $\#_{k}$ finito, existe $\varepsilon>0$ tal que, para cada $i \in I$, existem pareamentos $\left\langle\alpha_{i}^{-},{\alpha_{i}^{-{ }^{\prime}}}\right\rangle,\left\langle\alpha_{i}^{+}, \alpha_{i}^{+^{\prime}}\right\rangle \in \mathcal{P}$ que satisfazem o seguinte:

1. $\beta_{i}^{-}:=\left(a_{i}^{-}-\varepsilon, a_{i}^{-}\right) \subset \alpha_{i}^{-}$, sendo $a_{i}^{-}$uma extremidade de $\alpha_{i}^{-}$.

2. $\beta_{i}^{+}:=\left(a_{i}^{+}, a_{i}^{+}+\varepsilon\right) \subset \alpha_{i}^{+}$, sendo $a_{i}^{+}$uma extremidade de $\alpha_{i}^{+}$.

3. $\beta_{i}^{+} \cap \pi_{\mathcal{P}}^{-1}\left(D_{k}\right)=\emptyset$ e $\beta_{i}^{-} \cap \pi_{\mathcal{P}}^{-1}\left(D_{k}\right)=\emptyset$.

Denote-se por $\left\langle\beta_{i}^{+}, \beta_{i}^{+^{\prime}}\right\rangle,\left\langle\beta_{i}^{-}, \beta_{i}^{-^{\prime}}\right\rangle$ os respectivos subpareamentos de $\left\langle\alpha_{i}^{+}, \alpha_{i}^{+^{\prime}}\right\rangle$ e $\left\langle\alpha_{i}^{-}, \alpha_{i}^{-^{\prime}}\right\rangle$,

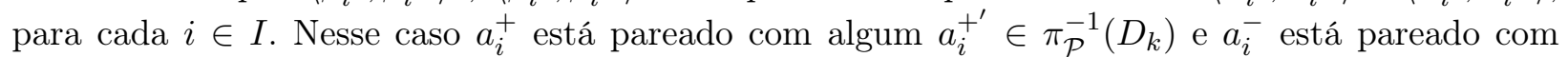
$\operatorname{algum}{a_{i}^{-}}^{\prime} \in \pi_{\mathcal{P}}^{-1}\left(D_{k}\right)$.

Dado qualquer $1 \leq l \leq \#_{k}$ considere-se o subespaço $\partial P \backslash\left(\beta_{i_{l}}^{+} \cup \beta_{i_{l}}^{+^{\prime}}\right)$, o qual contém $\pi_{\mathcal{P}}^{-1}\left(D_{k}\right)$. Éste, a sua vez é a união disjunta de dois arcos fechados $T_{1}$ e $T_{2}$ com $L_{i_{l}} \subset T_{2}$. Observe-se que $\beta_{i_{l}}^{+^{\prime}}=\beta_{i_{s}}^{-^{\prime}}$ para algum $1 \leq s \leq \#_{k}$, com o qual $L_{i_{s}} \subset T_{2}$ pois, no caso contrario, existiriam $z \in T_{1}$ e $z^{\prime} \in T_{2}$ tal que $d^{\mathcal{P}}\left(z, z^{\prime}\right)=0$, logo o par de pares $\left\{z, z^{\prime}\right\}$ e $\left\{b_{i_{l}}^{+}, \beta_{i_{l}}^{-}\right\}$seriam $\sim_{\mathcal{P}}$-vinculados, o qual é uma contradição. Portanto $i_{s}=i_{l+1}$ e assim $a_{i_{l}}^{+} \sim_{\mathcal{P}} a_{i_{l+1}}^{-}$.

Observação 3.4.6. Para $k \geq K_{0}$, o valor $\varepsilon>0$ usado na demostração do lema anterior vai ser maior ou igual que $e_{k}$, com o qual, $\left(a_{i_{l}}^{+}+r\right) \mathcal{P}\left(a_{i_{l+1}}^{-}-r\right)$, para todo $0<r<e_{k}$ e $1 \leq l \leq \#_{k}$.

A seguir serão construido os aneis $A_{k}^{t} \subset S_{\infty}$ necessarios para aplicar o Lema 3.2.2 e conseguir uma estimativa superior de $\ell_{\infty}\left(\gamma_{k}\right)$. Como $e_{k} \rightarrow 0$ quando $k \rightarrow+\infty$, pode-se tomar $K_{0}$ suficientemente grande de maneira que, para $k \geq K_{0}$, a distância de um lado de $P$ a qualquer outro lado de $P$ que não seja adjacente a este seja maior ou igual a $2 e_{k}$. Lembre-se que cada componente de $\pi_{\mathcal{P}}^{-1}\left(D_{k}\right)$ está contido no máximo em dois lados consecutivos de $P$ pelo qual, se $L_{j}$ é uma componente de $\pi_{\mathcal{P}}^{-1}\left(D_{k}\right)$ e se $T_{1}$ e $T_{2}$ são dois lados consecutivos que contém à $L_{j}$, então não perda de generalidade em supor que para qualquer outra componente $L_{i}$ de $\pi_{\mathcal{P}}^{-1}\left(D_{k}\right)$ que não esté contenido em $T_{1} \cup T_{2}$, tem-se $d_{P}\left(L_{i}, L_{j}\right) \geq 2 e_{k}$.

Sejam $T_{1}$ e $T_{2}$ dois lados de $P$ consecutivos. Dado $k \geq K_{0}$, suponha que existe $j \in I_{4}$ tal que $L_{j} \subset T_{1} \cup T_{2}$, $\log$ o o vértice $v_{j}$ é o vértice común entre $T_{1}$ e $T_{2}$ e $L_{j}=\left[a_{j}^{-}, a_{j}^{+}\right]=\left[a_{j}^{-}, v_{j}\right] \cup\left[v_{j}, a_{j}^{+}\right]$. Na figura 3.14, se $a_{j}$ denota a extremidade de $L_{j}$ que está mais perto do vértice $v_{j}$ e $M$ é o ponto de interseção da bissetriz de $v_{j}$ com a perpendicular ao lado de $P$ que contém o ponto $a_{j}$ e pasa por $a_{j}$, tem-se:

$$
d_{P}\left(a_{j}, M\right)=d_{P}\left(a_{j}, v_{j}\right) \cdot \tan \frac{\phi_{j}}{2} \geq 2 f_{k}
$$

Assim, para todo $x \in T_{1} \backslash L_{j}, d_{P}\left(x, T_{2}\right) \geq 2 f_{k}$ e igualmente, para todo $y \in T_{2} \backslash L_{j}$, tem-se $d_{P}\left(y, T_{1}\right) \geq 2 f_{k}$. Em particular, se $L_{i}$ é outra componente de $\pi_{\mathcal{P}}^{-1}\left(D_{k}\right)$ que intersecta o lado $T_{1}$ ou o lado $T_{2}$, então $d_{P}\left(L_{i}, L_{j}\right) \geq 2 h_{k}$.

Se existe $j \in I_{3}$ tal que $L_{j} \subset T_{1} \cup T_{2}$, é fácil ver que para $x \in T_{1} \backslash L_{j}$ e $y \in T_{2} \backslash L_{j}$, tem-se $d_{P}\left(x, L_{j}\right)=d_{P}\left(x, a_{j}^{-}\right)$e $d_{P}\left(y, L_{j}\right)=d_{P}\left(a_{j}^{+}, y\right)$. Logo para qualquer componente $L_{i}$ de $\pi_{\mathcal{P}}^{-1}\left(D_{k}\right)$ que intersecta o lado $T_{1}$ ou o lado $T_{2}, d_{P}\left(L_{i}, L_{j}\right) \geq 2 h_{k}$. 


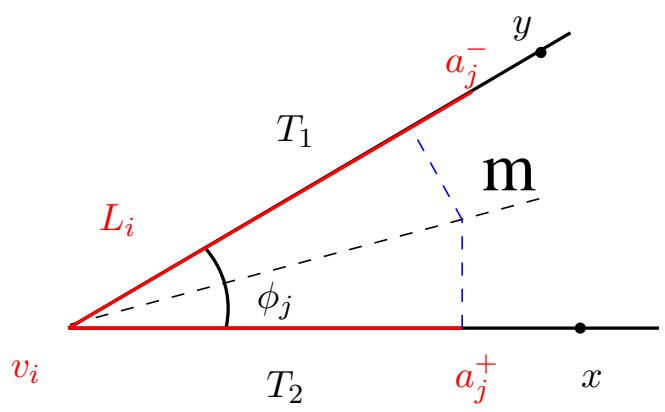

Figura 3.14

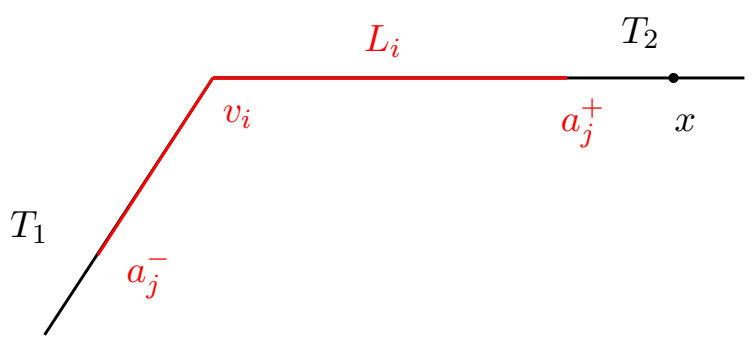

Figura 3.15

Suponha agora que não existe $j \in I_{3} \cup I_{4}$ tal que $L_{j} \subset T_{1} \cup T_{2}$ e seja $v$ o vértice común entre os lados $T_{1}$ e $T_{2}$. Sejam $j_{1}, j_{2} \in I$ (se existem!) tais que $a_{j_{1}}^{+}$é a extremidade direita de uma componente de $\pi_{\mathcal{P}}^{-1}\left(D_{k}\right)$ contida em $T_{1}$ más próxima de $v$, e $a_{j_{2}}^{-}$é a extremidade esquerda de uma componente de $\pi_{\mathcal{P}}^{-1}\left(D_{k}\right)$ contida em $T_{2}$ más próxima de $v$, ver figura 3.16 .

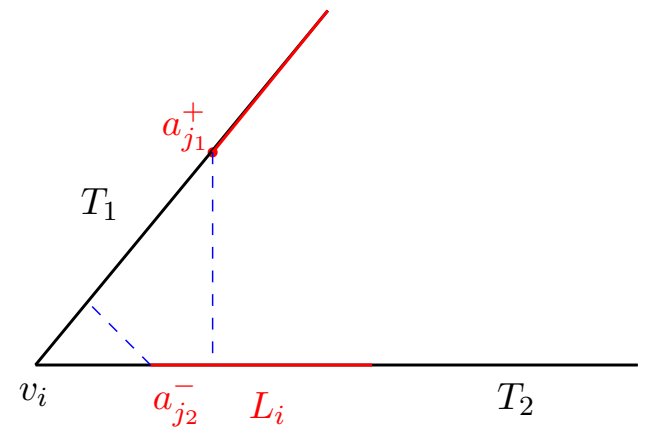

Figura 3.16

Como $D_{k} \supset D_{k+1}$ para todo $k$ e como $e_{k} \rightarrow 0$ quando $k \rightarrow+\infty$, escolha-se $K_{0}$ maior de ser necessario tal que para $k \geq K_{0}, d_{P}\left(T_{1} \cap \pi_{\mathcal{P}}^{-1}\left(D_{k}\right), T_{2}\right) \geq 2 e_{k}$ e $d_{P}\left(T_{2} \cap \pi_{\mathcal{P}}^{-1}\left(D_{k}\right), T_{1}\right) \geq 2 e_{k}$. Logo, neste caso tem-se também que $d_{P}\left(L_{i}, L_{j}\right) \geq 2 e_{k}$ se $L_{i}$ é uma componente de $\pi_{\mathcal{P}}^{-1}\left(D_{k}\right)$ que intersecta à $T_{1}$ e $L_{j}$ é uma componente de $\pi_{\mathcal{P}}^{-1}\left(D_{k}\right)$ que intersecta à $T_{2}$. Como $h_{k}=\min \left\{e_{k}, f_{k}\right\}$ conclui-se o seguinte.

Lema 3.4.7. Existe $K_{0} \geq 1$ tal que para $k \geq K_{0}$, se $L_{i}$ e $L_{j}$ são dois componentes diferentes de $\pi_{\mathcal{P}}^{-1}\left(D_{k}\right)$, então:

$$
d_{P}\left(L_{i}, L_{j}\right) \geq 2 h_{k}
$$

Com o qual $B_{d_{P}}\left(L_{i}, h_{k}\right) \cap B_{d_{P}}\left(L_{j}, h_{k}\right)=\emptyset$. 
A continuação provaremos que as diferentes bolas $B_{d_{P}}\left(L_{i}, h\right)$ são $\pi_{\mathcal{P}}$-colados num disco em $S_{\mathcal{P}}$.

Lema 3.4.8. Para $k \geq K_{0}$ e $0<h<h_{k}, B_{S_{\mathcal{P}}}\left(D_{k}, h\right)$ é um disco topológico. Além disso,

$$
B_{S_{\mathcal{P}}}\left(D_{k}, h\right)=\pi_{\mathcal{P}}\left(\bigsqcup_{i \in I} B_{d_{P}}\left(L_{i}, h\right)\right)
$$

Demonstração. Claramente $\pi_{\mathcal{P}}\left(\bigsqcup_{i \in I} B_{d_{P}}\left(L_{i}, h\right)\right) \subset B_{S_{\mathcal{P}}}\left(D_{k}, h\right)$. Se $z \notin B_{S_{\mathcal{P}}}\left(D_{k}, h\right)$ e $x \in \pi_{\mathcal{P}}^{-1}(z)$, então $d_{P}\left(x, \sqcup_{i \in I} L_{i}\right) \geq h$ : Qualquer $\mathcal{P}$-itinerário que conecte o ponto $z$ com $\pi_{\mathcal{P}}^{-1}\left(D_{k}\right)$ terá um $d_{P^{-}}$ custo maior ou igual que $h$. Assim tem-se a igualdade 3.4.

Para $0<r<h$ e $1 \leq l \leq \#_{k}$, denote-se por $\mathbb{S}_{l}(r)$ o conjunto:

$$
\mathbb{S}_{l}(r):=\mathbb{S}_{P}\left(L_{i_{l}}, r\right)=\left\{z \in P / d_{P}\left(z, L_{i_{l}}\right)=r\right\}
$$

Logo $\mathbb{S}_{l}(r)$ é um arco simple em $P$ cujo interior está no interior do polígono $P$ e suas extremidades estão em $\partial P$, sendo a extremidade esquerda o ponto $a_{i_{l}}^{-}-r$ e a extremidade direita o ponto $a_{i_{l}}^{+}+r$. Como consequência do Lema 3.4.5 (ver também a observação 3.4.6), a extremidade direita $a_{i_{l}}^{+}+r$ de $\mathbb{S}_{l}(r)$ é colado com a extremidade esquerda $a_{i_{l+1}}^{-}-r$ de $\mathbb{S}_{l+1}(r)$, para todo $1 \leq l \leq \#_{k}$. Portanto, sendo $\mathbb{S}_{l_{1}}(r)$ e $\mathbb{S}_{l_{2}}(r)$ conjuntos disjuntos quando $l_{1} \neq l_{2}$ (Lema 3.4.7), então o conjunto $\pi_{\mathcal{P}}\left(\mathbb{S}_{P}\left(\pi_{\mathcal{P}}^{-1}\left(D_{k}\right), r\right)\right)=\pi_{\mathcal{P}}\left(\bigsqcup_{l=1}^{\#_{k}} \mathbb{S}_{l}(r)\right)$ é homeomorfo a um círculo.

Sendo $B_{S_{\mathcal{P}}}\left(D_{k}, h\right)=D_{k} \sqcup \bigsqcup_{0<r<h} \pi_{\mathcal{P}}\left(\mathbb{S}_{P}\left(\pi_{\mathcal{P}}^{-1}\left(D_{k}\right), r\right)\right)$, conclui-se que a bola $B_{S_{\mathcal{P}}}\left(D_{k}, h\right)$ é em efeito homeomorfo a um disco.

Na figura 3.9 já foram mostrados os desenho das bolas $B_{d_{P}}\left(L_{i}, h\right)$ nos casos de componentes do tipo 1, 2 e 3, para $h \leq h_{k}$. Nos três casos pode-se ver que o disco divide-se facilmente em peças circulares e peças retangulares. Como foi definido o parámetro $f_{k}$, observe-se da figura 3.17 que quando $L_{i}$ é uma componente do tipo 4 , a bola $B_{d_{P}}\left(L_{i}, h\right)$ divide-se também em peças que não sobrepõem uns aos outros: dois peças circulares e uma peça "retangular"no meio.

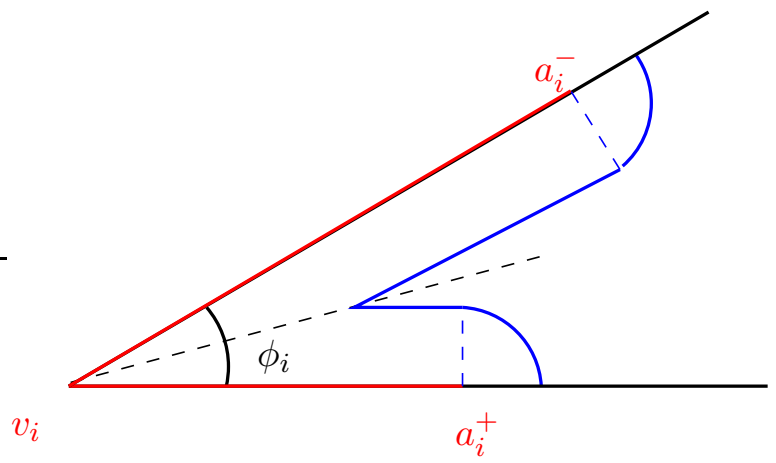

Figura 3.17: Desenho do disco $B_{d_{P}}\left(L_{i}, h\right)$ para $i \in I_{4}$ e $h \leq f_{k}$.

Indiferentemente do tipo da componente $L_{i}$ de $\pi_{\mathcal{P}}^{-1}\left(D_{k}\right)$, queremos pensar no disco $B_{d_{P}}\left(L_{i}, h\right)$ como a união de cuatro peças: uma circular na esquerda $C_{i,-}$, uma circular direita $C_{i,+}$, uma peça circular central $C_{i, 0}$ e uma retangular $R_{i}$, onde,

1) Se $L_{i}$ é do tipo 1, a peça retangular tem comprimento 0 , é dizer, é um segmento radial. 
2) Se $L_{i}$ é do tipo 2, a peça circular central tem ângulo zero, logo também é um segmento, e se algumas das extremidades de $L_{i}$ é um vértice do $P$ de ângulo menor que $\frac{\pi}{2}$, a peça circular correspondente também tem ângulo zero.

3) Se $L_{i}$ é do tipo 3, neste caso existem as cuatro peças, mas a peça retangular consiste na união de dois retangulos $R_{i}=R_{i,-} \cup R_{i,+}$; a peça circular central $C_{i, 0}$ está entre $R_{i,-}$ e $R_{i,+}$.

4) Se $L_{i}$ é do tipo 4, igual que no ítem 2), a peça circular central tem ângulo zero. A peça retangular $R_{i}$ na verdade é a união de dos trapézios $R_{i,-}$ e $R_{i,+}$.

Tome-se $K_{0}$ suficientemente grande de forma que todos os lemas e observações precedentes sejam válidos. Dado $k \geq K_{0}$ e $t>1$, defina-se o conjunto $A_{k}^{t}$ como:

$$
A_{k}^{t}:=B_{d^{\mathcal{P}}}\left(D_{k}, h_{k}\right) \backslash \bar{B}_{d^{\mathcal{P}}}\left(D_{k}, \frac{h_{k}}{t^{\#}}\right)
$$

A Proposição 3.4.8 garante que o conjunto $A_{k}^{t}$ é um anel. Este será a união das $\pi_{\mathcal{P}}$-projeções dos conjuntos $B_{P}\left(L_{i}, h_{k}\right) \backslash \bar{B}_{P}\left(L_{i}, \frac{h_{k}}{t^{\#}}\right), i \in I$. Igual que acima, consideraremos cada um destos pedaços divididos em cuatro peças: Três circulares $C_{i,-}^{t}, C_{i, 0}^{t}$ e $C_{i,+}^{t}$, e uma retangular $R_{i}^{t}$. As figuras 3.18 e 3.19 mostram um desenho das diferentes peças. Por outro lado, como $h_{k} \leq e_{k}$, tem-se que $A_{k}^{t} \subset S_{\infty}$ é redutível à curva geodésica $\gamma_{k}$, para cada $k \geq K_{0}$.
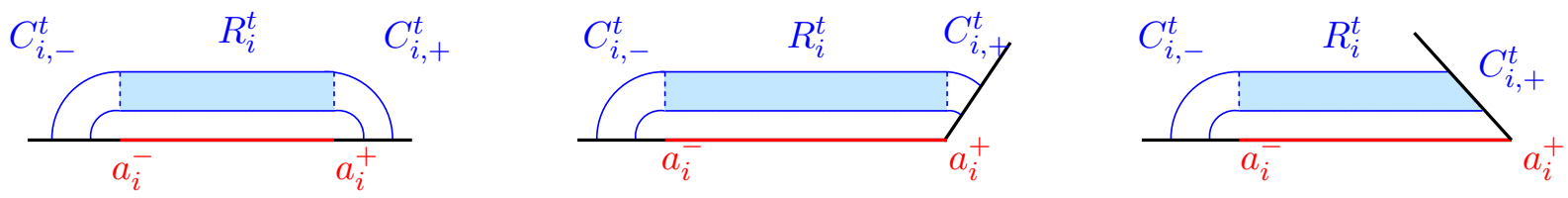

Figura 3.18: Semianel para uma componente $L_{i}$ do tipo 2
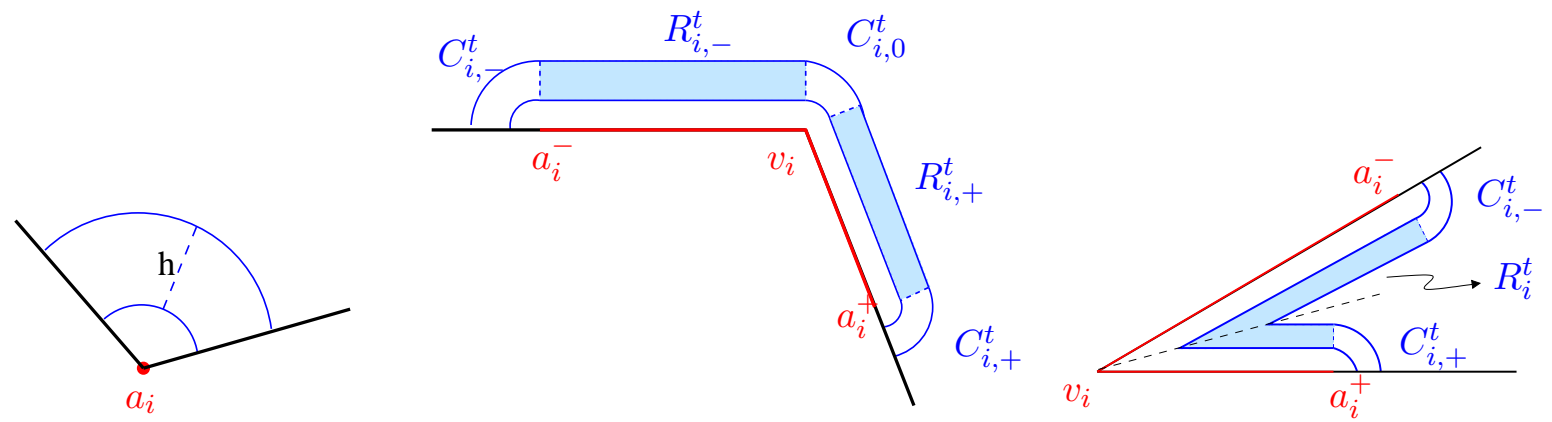

Figura 3.19: Semianel para uma componente $L_{i}$ do tipo 1,3 e 4 respectivamente

Até agora foi tratado somente o caso onde $P$ é um polígono. No caso geral onde $P$ é um multipolígono, escolha-se um árvore de extensão do grafo $\Upsilon_{\mathcal{P}}$ como foi explicado na seção 2.3 de forma que todos os polígonos de $P$ sejam colados num disco poligonal $P^{\prime}$; suponha que $\delta_{1}, \ldots, \delta_{s}$ são os arcos contidos no interior de $P^{\prime}$ dos pareamentos correspondentes as arestas do árvore $\Upsilon_{\mathcal{P}}$. Como $m_{k} \rightarrow 0$ quando $k \rightarrow+\infty$, pode-se assumir sem perda de generalidade que $\pi_{\mathcal{P}}^{-1}\left(D_{k}\right) \subset \partial P^{\prime}$ para $k \geq K_{0}$. Todos os lemas desenvolvidos nesta seção valem também para os espaço $\left(P^{\prime}, d_{P^{\prime}}\right)$ e $P^{\prime} / \mathcal{P}^{\prime}$. Observe-se que no caso do Lema 3.4.3, se $\pi_{\mathcal{P}}^{-1}\left(D_{k}\right)$ têm brechas (como na figura 3.13), estes devem estar no conjunto $\left\{\operatorname{int} \delta_{1}, \operatorname{int} \delta_{2}, \ldots, \operatorname{int} \delta_{s}\right\}$, com o qual conclui-se que tomando $K_{0}$ suficientemente grande, as brechas de $\pi_{\mathcal{P}}^{-1}\left(D_{k}\right)$ serão as mesmas para todo $k \geq K_{0}$ e o minimo comprimento de uma brecha é maior que $2 \cdot e_{k}$. Em consequência, elas não serão um problema para a construção dos aneis $A_{k}^{t}$. 
Demostração do Teorema 3.3.2. Considere-se $k \geq K_{0}$. Seja $\rho_{k}^{t}(z)|d z|$ a densidade Boreliana sobre o anel $A_{k}^{t}$ definido por:

$$
\rho_{k}^{t}(z):=\left\{\begin{array}{ccc}
\frac{1}{d^{\mathcal{P}}\left(z, D_{k}\right)} ; & \text { se } & \pi_{\mathcal{P}}^{-1}(z) \in \bigcup_{l=1}^{\# k}\left(C_{i_{l},-}^{t} \cup C_{i_{l}, 0}^{t} \cup C_{i_{l},+}^{t}\right) \\
\frac{\#_{k} \cdot \log t}{h_{k}\left(1-\frac{1}{t^{\#} k}\right)} ; & \text { se } & \pi_{\mathcal{P}}^{-1}(z) \in \bigcup_{l=1}^{\#_{k}} R_{i_{l}}^{t}
\end{array}\right.
$$

Onde para todo $1 \leq l \leq \#_{k}, R_{i_{l}}^{t}=R_{i_{l}} \cap A_{k}^{t}$ e $C_{i_{l}, p}^{t}=C_{i_{l}, p}^{t} \cap A_{K}^{t}$, sendo $p=-, 0,+$.

O mesmo argumento usado no Lema 3.2.3 mostra que,

$$
\mathcal{L}_{\rho_{k}^{t}}\left(A_{k}^{t}\right)=\#_{k} \cdot \log t
$$

Similarmente é fácil ver que para cada $1 \leq l \leq \#_{k}$, a $\rho_{k}^{t}$-área de uma peça circular $C_{i_{l}, p}^{t}$, $p=-, 0,+$, satisfaz a desigualdade:

$$
\mathcal{A}_{\rho_{k}^{t}}\left(C_{i_{l}, p}^{t}\right) \leq 2 \pi \cdot \#_{k} \cdot \log t
$$

Com relação a $\rho_{k}^{t}$-área de cada peça retangular $R_{i_{l}}^{t}$, tem-se o seguinte:

1) Se $i_{l} \in I_{1}, \mathcal{A}_{\rho_{k}^{t}}\left(R_{i_{l}}^{t}\right)=0$.

2) Se $i_{l} \in I_{2}, \mathcal{A}_{\rho_{k}^{t}}\left(R_{i_{l}}^{t}\right) \leq \frac{\#_{k}^{2} \log ^{2} t}{h_{k}^{2}\left(1-\frac{1}{t^{\#}}\right)^{2}} \cdot h_{k}\left(1-\frac{1}{t^{\# k}}\right) \cdot m_{\partial P}\left(L_{i_{l}}\right)=\frac{\#_{k}^{2} \log ^{2} t}{h_{k}\left(1-\frac{1}{t^{\# k}}\right)} \cdot m_{\partial P}\left(L_{i_{l}}\right)$.

3) Se $i_{l} \in I_{3}, \mathcal{A}_{\rho_{k}^{t}}\left(R_{i_{l}}^{t}\right)=\mathcal{A}_{\rho_{k}^{t}}\left(R_{i_{l},-}^{t}\right)+\mathcal{A}_{\rho_{k}^{t}}\left(R_{i_{l},+}^{t}\right)=\frac{\#_{k}^{2} \log ^{2} t}{h_{k}\left(1-\frac{1}{t^{\#}}\right)} \cdot m_{\partial P}\left(L_{i_{l}}\right)$.

4) Se $i_{l} \in I_{4}$, ao ver a figura 3.20 , é facil ver que $\mathcal{A}_{\rho_{k}^{t}}\left(R_{i_{l}}^{t}\right) \leq \frac{\#_{k}^{2} \log ^{2} t}{h_{k}\left(1-\frac{1}{t^{\# k}}\right)} \cdot m_{\partial P}\left(L_{i_{l}}\right)$.

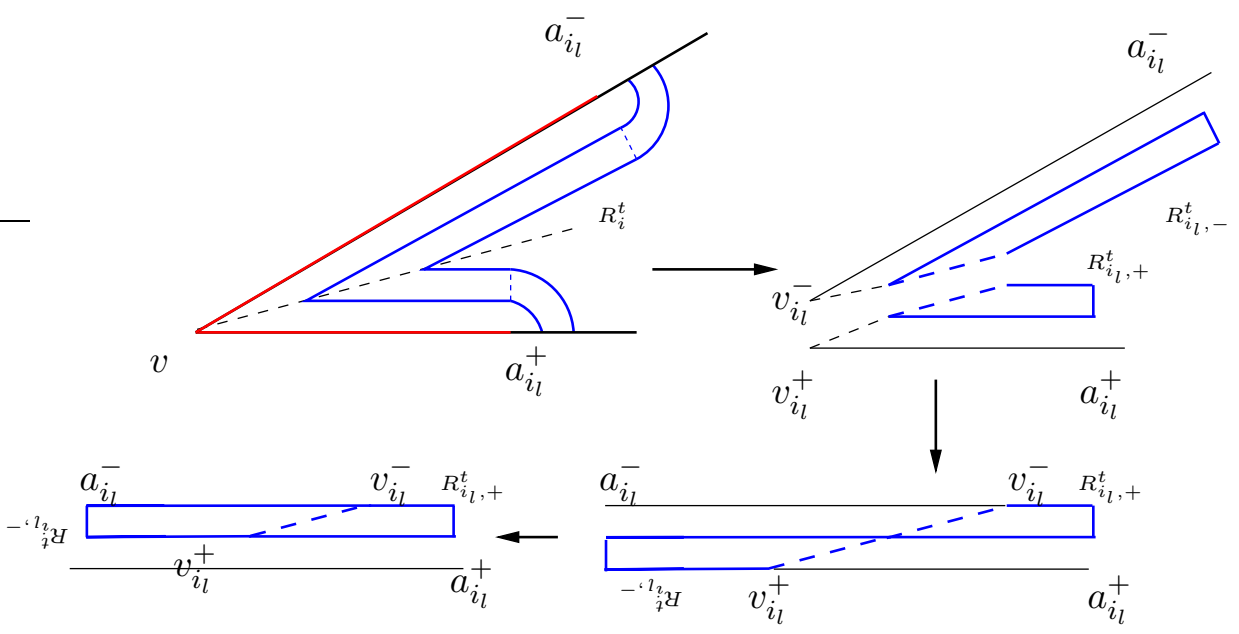

Figura 3.20: Transformação da peça retangular $R_{i}^{t}$ num retangulo de verdade.

Como $m_{k}=m_{G_{\mathcal{P}}}\left(D_{k}\right)=\sum_{l=1}^{\#_{k}} m_{\partial P}\left(L_{i_{l}}\right)$, então, 


$$
\mathcal{A}_{\rho_{k}^{t}}\left(\bigcup_{l=1}^{\#_{k}}\left(R_{i_{l}}^{t}\right)\right) \leq \frac{\#_{k}^{2} \log ^{2} t}{h_{k}\left(1-\frac{1}{t^{\#}}\right)} \cdot m_{k}
$$

Como por cada componente $L_{i}$ tem-se três peças circulares, então existem em total $3 \cdot \#_{k}$ peças circulares, com o qual,

$$
\mathcal{A}_{\rho_{k}^{t}}\left(\bigcup_{l=1}^{\#_{k}} C_{i_{l}, p}^{t}\right) \leq 3 \cdot \#_{k} \cdot 2 \pi \cdot \#_{k} \cdot \log t=6 \pi \cdot \#_{k}^{2} \cdot \log t
$$

Aplicando o Lema 3.2.2 tem-se para todo $t>1$ que,

$$
\begin{aligned}
\ell_{\infty}\left(\gamma_{k}\right) & \leq \pi \cdot \frac{\mathcal{A}_{\rho_{k}^{t}}\left(A_{k}^{t}\right)}{\mathcal{L}_{\rho_{k}^{t}}\left(A_{k}^{t}\right)^{2}} \\
& \leq \frac{\pi}{\#_{k}^{2} \cdot \log ^{2} t}\left(\frac{\#_{k}^{2} \log ^{2} t}{h_{k}\left(1-\frac{1}{t^{\#}}\right)} \cdot m_{k}+6 \pi \cdot \#_{k}^{2} \cdot \log t\right) \\
& \leq \frac{\pi}{1-\frac{1}{t^{\#}}} \frac{m_{k}}{h_{k}}+\frac{6 \pi^{2}}{\log t}
\end{aligned}
$$

Ao fazer $t \rightarrow+\infty$ na desigualdade acima, conclui-se que,

$$
\ell_{\infty}\left(\gamma_{k}\right) \leq \pi \cdot \frac{m_{k}}{h_{k}}
$$

\subsection{Estimativas inferiores das coordenadas de comprimento}

Considere-se novamente a esfera furada $S_{\infty}$ do modelo base. Na seção 3.2 foi estimado cotas superiores para $\ell_{\infty}\left(\gamma_{k}\right), k \geq 1$. Gostariamos agora calcular estimativas inferiores para os comprimentos hiperbólicos das mesmas geodésicas. Para conseguir isto basta estimar por acima o comprimento hiperbólico de outra geodésica que intersecte transversalmente a curva $\gamma_{k}$ : Se $\beta$ é uma curva geodésica fechada simples que intersecta à $\gamma_{k}$, então $\gamma_{k}$ deve atravessar o colar $\mathbb{A}_{\beta}$ de $\beta$, com o qual,

$$
\ell_{\infty}\left(\gamma_{k}\right) \geq 2 i\left(\gamma_{k}, \beta\right) \cdot \eta\left(\ell_{\infty}(\beta)\right),
$$

onde $i\left(\gamma_{k}, \beta\right)$ denota o número de intersećão das curvas $\gamma_{k}$ e $\beta$. Assim, se $M$ é uma estimativa superior de $\ell_{\infty}(\beta)$, é dizer, se $\ell_{\infty}(\beta) \leq M$, como a função colar $\eta$ é estritamente decrescente, tem-se

$$
\ell_{\infty}\left(\gamma_{k}\right) \geq 2 i\left(\gamma_{k}, \beta\right) \cdot \eta(M)
$$

Fixe um inteiro $k \geq 1$ e considere-se a família de curvas fechadas simples $\hat{\beta}_{k, l}, l \geq 1$, em $S_{\infty}$ mostradas na figura $3.4, \log$ tome-se para cada $l \geq 1$ a curva geodésica $\beta_{k, l}$ na classe de homotopía da curva $\hat{\beta}_{k, l}$. Observe-se que cada $\beta_{k, l}$ separa em $S_{\mathcal{P}}$ o conjunto $\left\{p_{k}, p_{k+1}, \ldots, p_{k+l}\right\}$ do ponto irregular $q$ e do resto de pontos cônicos, isto é, do conjunto $\{q\} \cup\left\{p_{-1}, p_{0}, p_{1}, \ldots, p_{k-1}\right\} \cup$ $\left\{p_{k+l+1}, p_{k+l+2}, \ldots \ldots\right\}$.

As curvas $\left(\hat{\beta}_{k, l}\right)_{l \geq 1}$ podem ser escolhidas de forma que todas elas sejam mutuamente disjuntas, $\operatorname{logo}$ pela Proposição $1.3 .1 \mathrm{v}$ ), tem-se que para $1 \leq l_{1}<l_{2}, \beta_{k, l_{1}} \cap \beta_{k, l_{2}}=\emptyset$. Por outro lado cada uma das curvas $\hat{\beta}_{k, l}$ intersecta à $\gamma_{k}$ em dois pontos, pelo qual da mesma Proposição 1.3.1 


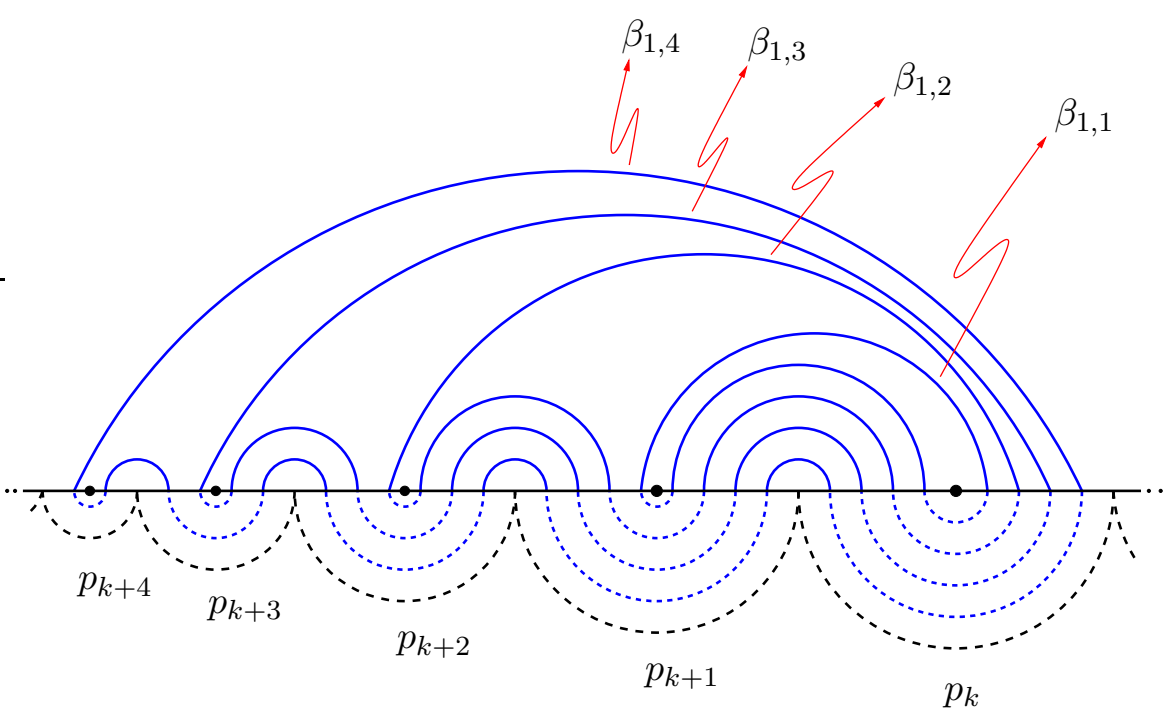

Figura 3.21: Desenho das curvas $\beta_{k, l}$

$\mathrm{v}), i\left(\gamma_{k}, \beta_{k, l}\right)=2$, para todo $k, l \geq 1$. A figura 3.22 mostra um possível desenho das primeiras geodésicas $\beta_{k, l}$ em $S_{\infty}$.

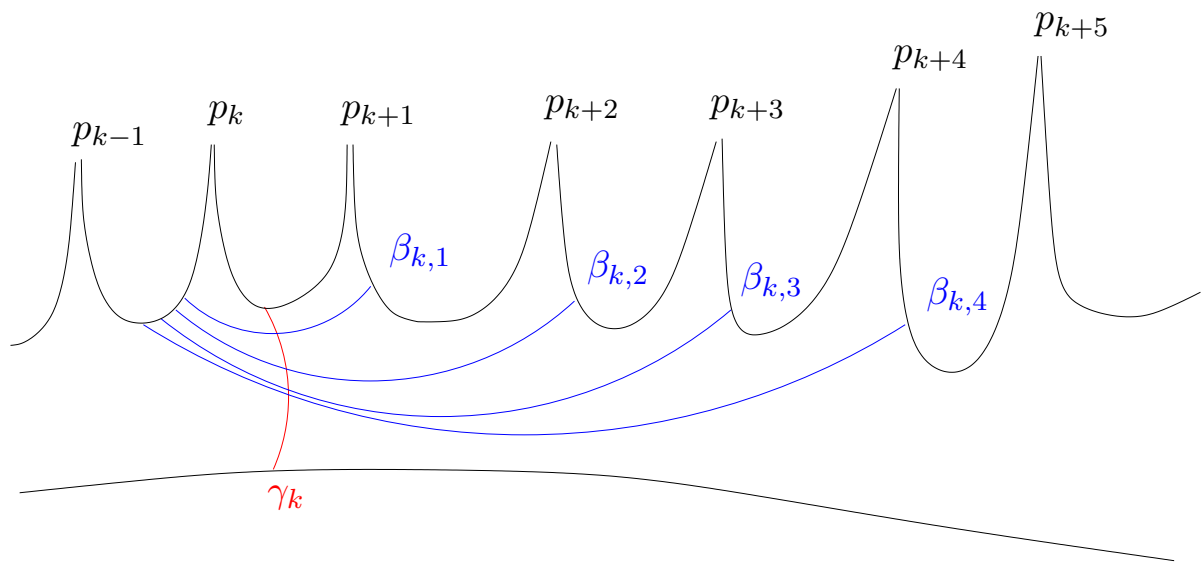

Figura 3.22: Curvas geod́esica $\beta_{k, l}$, para $l=1,2,3,4$.

Pelo Lema do colar, ver Teorema 1.3.5, para cada $l_{1}, l_{2} \geq 1$ com $l_{1} \neq l_{2}$, tem-se que os colares $\mathbb{A}_{\beta_{k, l_{1}}}$ e $\mathbb{A}_{\beta_{k, l_{2}}}$ são disjuntos. Dado que $\gamma_{k}$ atravessa cada um destos colares tem-se a seguinte desigualdade,

$$
\ell_{\infty}\left(\gamma_{k}\right) \geq 4 \sum_{l \geq 1} \eta\left(\ell_{\infty}\left(\beta_{k, l}\right)\right)
$$

Claramente basta somente uma estimativa superior do comprimento de uma só das curvas $\beta_{k, l}$ para obter a uma estimativa inferior de $\ell_{\infty}\left(\gamma_{k}\right)$, porém em principio, a soma infinita acima daria uma estimativa inferior melhor. vamos prosseguir em seguida com o calculo de estimativas superiores para cada $\ell_{\infty}\left(\beta_{k, l}\right)$. Dado $t>1$, denote-se por $x_{1}^{t}, x_{2}^{t}, x_{3}^{t}, z_{1}^{t}, z_{2}^{t}, z_{3}^{t}, z_{4}^{t} \in P$ os seguintes pontos:

$$
\begin{aligned}
& x_{1}^{t}:=2 \sum_{n>k+l} a_{n}+\frac{a_{k+l}}{t^{l}}, \quad x_{2}^{t}:=2 \sum_{n \geq k} a_{n}-a_{k+l}, \quad x_{3}^{t}:=2 \sum_{n \geq k} a_{n}-\frac{a_{k+l}}{t^{l}}, \\
& z_{1}^{t}:=x_{1}^{t}+2 a_{k+l} \cdot i, \quad z_{2}^{t}:=x_{2}^{t}+a_{k+l} \cdot i, \quad z_{3}^{t}:=x_{3}^{t}+2 a_{k+l} \cdot i, \quad z_{4}^{t}:=\hat{p}_{k+l}+a_{k+l} \cdot i,
\end{aligned}
$$


onde $\hat{p}_{k+l}=\pi_{\mathcal{P}}^{-1}\left(p_{k+l}\right)$. Denote-se por $c_{j}, j=1, \ldots, l$, os pontos $c_{j}:=2 \sum_{n \geq k+j} a_{n}$, por $C_{j}^{t} \subset P \circ$ semi-anel,

$$
C_{j}^{t}:=\left\{z \in P / \frac{a_{k+l}}{t^{l}}<\left|z-c_{j}\right|<a_{k+l}\right\},
$$

e por $R^{t}$ a região aberta em $P$ limitada pela poligonal com vértices $x_{1}^{t}, z_{1}^{t}, z_{3}^{t}, x_{3}^{t}, x_{2}^{t}, z_{2}^{t}, z_{4}^{t}$ e $\hat{p}_{k+l}$. Ver a figura 3.23 para o caso $l=3$.

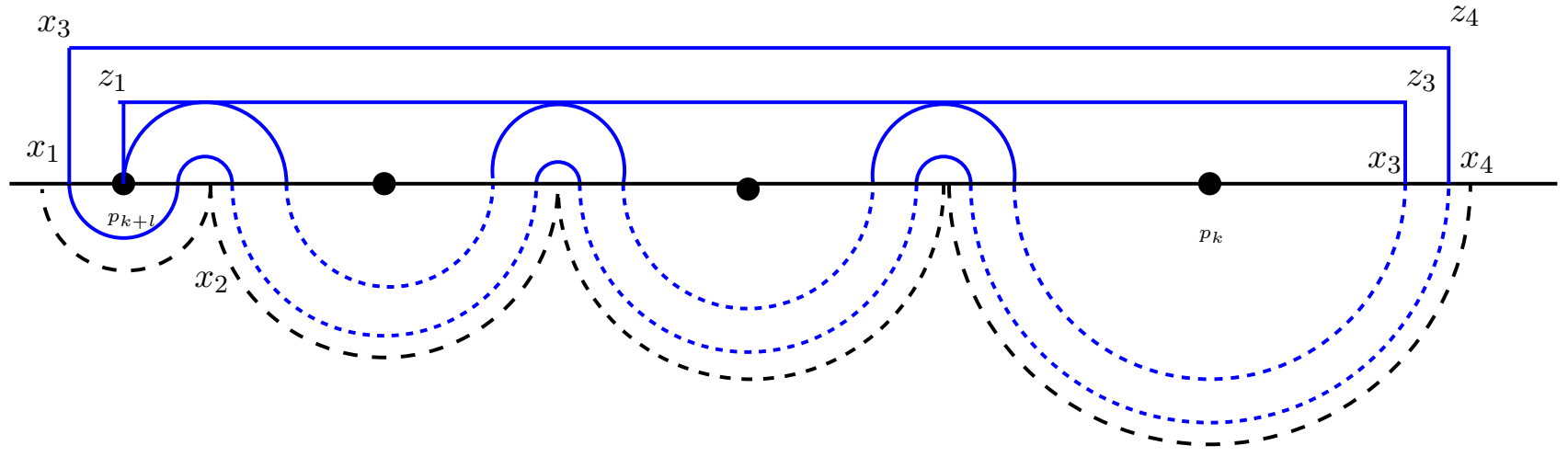

Figura 3.23: Desenho do anel $B_{k, 3, t}$.

Observe-se que $c_{1}, c_{2}, \ldots, c_{l} \in \pi_{\mathcal{P}}^{-1}(q)$. A união das regiões $R^{t} \cup \bigcup_{j=1}^{l} C_{j}^{t}$ é projetada por $\pi_{\mathcal{P}}$ num anel $B_{k, l, t} \subset S_{\infty}$ que separa os furos de $S_{\infty}$ do mesmo jeito que $\beta_{k, l}$. Considere-se em $B_{k, l, t}$ a densidade Boreliana $\rho_{k, l}^{t}(z)|d z|$ dada por:

$$
\rho_{k, l}^{t}(z):=\left\{\begin{array}{lll}
\frac{1}{d^{\mathcal{P}}\left(z, D_{k}\right)} ; & \text { se } & \pi_{\mathcal{P}}^{-1}(z) \in \bigcup_{j=1}^{l} C_{j}^{t} \\
\frac{l \cdot \log t}{a_{k+l}\left(1-\frac{1}{t^{l}}\right)} ; & \text { se } & \pi_{\mathcal{P}}^{-1}(z) \in R^{t}
\end{array}\right.
$$

Logo usando novamente a desigualdade 3.1 , tem-se $\ell_{\infty}\left(\beta_{k, l}\right) \leq \pi \cdot \frac{\mathcal{A}_{\rho_{k, l}^{t}}\left(B_{k, l, t}\right)}{\mathcal{L}_{\rho_{k, l}^{t}}\left(B_{k, l, t}\right)^{2}}$. O cálculo de $\mathcal{A}_{\rho_{k, l}^{t}}\left(B_{k, l, t}\right)$ e $\mathcal{L}_{\rho_{k, l}^{t}}\left(B_{k, l, t}\right)$ é similar aos feitos nos Lemas 3.2.3 e 3.2.4, pelo qual serão obviados os detalhes: $\mathcal{L}_{\rho_{k, l}^{t}}\left(B_{k, l, t}\right)=l \cdot \log t, \mathcal{A}_{\rho_{k, l}^{t}}\left(C_{j}^{t}\right)=\pi \cdot l \cdot \log t$, para $1 \leq j \leq l$ e, com relação à $R^{t}$,

$$
\begin{aligned}
\mathcal{A}_{\rho_{k, l}^{t}}\left(R^{t}\right) & =\frac{l^{2} \log ^{2} t}{a_{k+l}^{2}\left(1-\frac{1}{t^{l}}\right)^{2}}\left[2 a_{k+l}^{2}\left(1-\frac{1}{t^{l}}\right)+a_{k+l}\left(x_{3}^{t}-x_{1}^{t}\right)\right] \\
& =\frac{l^{2} \log ^{2} t}{a_{k+l}^{2}\left(1-\frac{1}{t^{l}}\right)^{2}}\left[2 a_{k+l}^{2}\left(1-\frac{1}{t^{l}}\right)+2 \sum_{n=k}^{k+l-1} a_{n}+2 a_{k+l}\left(1-\frac{1}{t^{l}}\right)\right] \\
& =l^{2} \log ^{2} t\left[\frac{4}{\left(1-\frac{1}{t^{l}}\right)}+\frac{2}{\left(1-\frac{1}{t^{l}}\right)^{2}} \cdot \frac{\sum_{k \leq n<n+k} a_{n}}{a_{k+l}}\right]
\end{aligned}
$$

$\operatorname{Logo} \mathcal{A}_{\rho_{k, l}^{t}}\left(B_{k, l, t}\right)=\mathcal{A}_{\rho_{k, l}^{t}}\left(R^{t}\right)+\pi \cdot l^{2} \log t$, pelo qual, para todo $k, l \geq 1, t>1$, 


$$
\begin{aligned}
\ell_{\infty}\left(\beta_{k, l}\right) \leq & \pi \cdot \frac{\mathcal{A}_{\rho_{k, l}^{t}}\left(B_{k, l, t}\right)}{\mathcal{L}_{\rho_{k, l}^{t}}\left(B_{k, l, t}\right)^{2}} \\
& \pi\left[\frac{4}{\left(1-\frac{1}{t^{l}}\right)}+\frac{2}{\left(1-\frac{1}{t^{l}}\right)^{2}} \cdot \frac{\sum_{k \leq n<n+k} a_{n}}{a_{k+l}}+\frac{\pi}{\log t}\right]
\end{aligned}
$$

Ao fazer $t \rightarrow+\infty$ na desigualdade acima, conclui-se o seguinte resultado.

Proposição 3.5.1. Na esfera de papel furada do modelo base,

$$
\ell_{\infty}\left(\beta_{k, l}\right) \leq 4 \pi+2 \pi \cdot \frac{1}{a_{k+l}} \sum_{n=k}^{k+l-1} a_{n},
$$

para todo $k, l \geq 1$. Em consequência, para cada $k \geq 1$,

$$
\ell_{\infty}\left(\gamma_{k}\right) \geq 4 \sum_{l \geq 1} \eta\left(4 \pi+2 \pi \cdot \frac{1}{a_{k+l}} \sum_{n=k}^{k+l-1} a_{n}\right)
$$

Exemplo 3.5.2. Suponha que $a_{n}=\lambda^{n}, n \geq 1$, para algum $0<\lambda<1$, como no exemplo 3.2.6. $\operatorname{Logo} \sum_{n=k}^{k+l-1} a_{n}=\lambda^{k} \cdot \frac{1-\lambda^{l}}{1-\lambda}$, pelo qual,

$$
\ell_{\infty}\left(\gamma_{k}\right) \geq 4 \sum_{l \geq 1} \eta\left(4 \pi+2 \pi \frac{1-\lambda^{l}}{\lambda^{l}(1-\lambda)}\right)
$$

Observe-se que a expresão na direita na desigualdade não depende do índice $k$, assim, junto com a estimativa superior do exemplo 3.2.6, tem-se,

$$
4 \sum_{l \geq 1} \eta\left(4 \pi+2 \pi \frac{1-\lambda^{l}}{\lambda^{l}(1-\lambda)}\right) \leq \ell_{\infty}\left(\gamma_{k}\right) \leq 2 \pi,
$$

para cada $k \geq 1$. No caso particular $a_{n}=\frac{1}{2^{n}}, n \geq 1$,

$$
6,97 \times 10^{-6} \approx 4 \sum_{l \geq 1} \log \operatorname{coth}\left(2^{l} \pi\right) \leq \ell_{\infty}\left(\gamma_{k}\right) \leq 2 \pi
$$

O próximo criterio permite determinar se a família $\left\{\ell_{\infty}\left(\gamma_{k}\right)\right\}_{k \geq 0}$ na esfera furada $S_{\infty}$ está limitada por constantes positivas.

Corolario 3.5.3. Se $0<\liminf _{k \rightarrow+\infty} \frac{a_{k+1}}{a_{k}} \leq \limsup _{k \rightarrow+\infty} \frac{a_{k+1}}{a_{k}}<1$, então existem $m, M>0$ tais que, para todo $k \geq 0$,

$$
m \leq \ell_{\infty}\left(\gamma_{k}\right) \leq M
$$


Em consequência, no espaço de Teichmüller $\mathcal{T}\left(S_{\infty}\right)$ as distâncias $d_{\mathcal{T}}$ e $d_{\mathcal{L}}$ são topológicamente equivalentes.

Demonstração. $\limsup _{k \rightarrow+\infty} \frac{a_{k+1}}{a_{k}}<1$ garante a existencia da constante $M<+\infty$ pela Proposição 3.2.9. Como consequência da proposição 3.5.1,

$$
\ell_{\infty}\left(\beta_{k, 1}\right) \leq 4 \pi+2 \pi \frac{a_{k}}{a_{k+1}}
$$

Mas como $\liminf _{k \rightarrow+\infty} \frac{a_{k+1}}{a_{k}}>0$, existe $m^{\prime}>0$ tal que $\sup _{k \geq 1} \frac{a_{k}}{a_{k+1}} \leq m^{\prime}$. Assim, para $k \geq 1$.

$$
\ell_{\infty}\left(\gamma_{k}\right) \geq \eta\left(4 \pi+2 \pi \frac{a_{k}}{a_{k+1}}\right) \geq m:=\eta\left(4 \pi+2 \pi m^{\prime}\right)
$$

O fato de que as distâncias $d_{\mathcal{T}}$ e $d_{\mathcal{L}}$ geram a mesma topología em $\mathcal{T}\left(S_{\infty}\right)$ é consequência imediata do Teorema de Shiga (ver o Teorema 1.3.12).

É tentador pensar em usar a Proposição 3.5.1 para determinar se a família $\left\{\ell_{\infty}\left(\gamma_{k}\right)\right\}_{k \geq 0}$ não está limitada superiormente (Por exemplo quando $a_{n} \asymp \frac{1}{n^{s}}$ ). Isso não será possível pela seguinte razão: Como $a_{n} \geq a_{n+1}$ para todo $n \geq 1$, então $\sum_{n=k}^{n+l-1} \frac{a_{n}}{a_{k+l}} \geq l-1$, logo,

$$
\sum_{l \geq 1} \eta\left(4 \pi+2 \pi \frac{1}{a_{k+l}} \sum_{n=k}^{k+l-1} a_{n}\right)<\sum_{l \geq 1} \eta(2 \pi+2 \pi l) \approx 1,7 \times 10^{-4}
$$

Portanto não é possível fazer que a soma na desigualdade da Proposição 3.5.1 diverga para infinito. ¿É possível conseguir melhores estimativas inferiores dos comprimentos das geodésica $\gamma_{k}$ no modelo base usando tal vez outro argumento?

O argumento anterior para conseguir estimativas inferiores dos comprimentos das curvas em $\Gamma_{\infty}$ no modelo base sirve também para obter estimativas inferiores das geodésicas da decomposição em calças de uma esfera de papel furada qualquer. Seja $S_{\mathcal{P}}$ uma esfera de papel com pontos irregulares $q^{1}, \ldots, q^{m}$. Suponha que queremos estimar por baixo o comprimento hiperbólico de $\gamma_{k}^{j}$ para $j=$ $1, \ldots, m$ e $k \geq 1$. Basta estimar por acima o comprimento hiperbólico de uma geodésica fechada simples em $\chi_{k, l}^{j} \subset S_{\infty}$ que separe os furos $p_{k}^{j}$ e $p_{k+l}^{j}$ do resto dos furos para algum $l \geq 1$, ver a figura 3.24, pois pelo Lema do colar, se $\ell_{\infty}\left(\chi_{k, l}^{j}\right) \leq M$, então,

$$
\ell_{\infty}\left(\gamma_{k}^{j}\right) \geq 4 \eta(M)
$$

Denote-se por $\hat{p}_{n}^{j}$ a $\pi_{\mathcal{P}}$-preimagem do ponto cônico $p_{n}^{j}$. Suponha que $\hat{p}_{k}^{j}$ e $\hat{p}_{k+l}^{j}$ estão contidos num mesmo lado do multipolígono $P$ e que $\left\langle\alpha_{k}, \alpha_{k}^{\prime}\right\rangle$ e $\left\langle\alpha_{k+l}, \alpha_{k+l}^{\prime}\right\rangle$ são os respectivos pareamentos cujos pontos de dobraduras são os pontos $p_{k}^{j}$ e $p_{k+l}^{j}$. Dado $t>0$ pequenho, considere-se o anel $A$ mostrado na figura 3.25 .

Aquí, se $a_{k, l}$ denota o mínimo entre $\left|\left\langle\alpha_{k}, \alpha_{k}^{\prime}\right\rangle\right|$ e $\left|\left\langle\alpha_{k+l}, \alpha_{k+l}^{\prime}\right\rangle\right|$, então,

$$
\begin{array}{r}
x_{1}:=\hat{p}_{k+l}^{j}-a_{k, l}, \quad x_{2}:=\hat{p}_{k+l}^{j}+a_{k, l}, \quad x_{3}:=\hat{p}_{k}^{j}-a_{k, l}, \quad x_{4}:=\hat{p}_{k}^{j}+a_{k, l}, \\
z_{1}:=x_{2}+i t, \quad z_{2}:=x_{3}+i t, \quad z_{3}:=\hat{p}_{k+l}^{j}+i\left(t+a_{k, l}\right), \\
z_{4}:=\hat{p}_{k}^{j}+i\left(t+a_{k, l}\right), \quad z_{5}:=x_{1}+i\left(t+2 a_{k, l}\right), \quad z_{6}:=x_{4}+i\left(t+2 a_{k, l}\right) .
\end{array}
$$




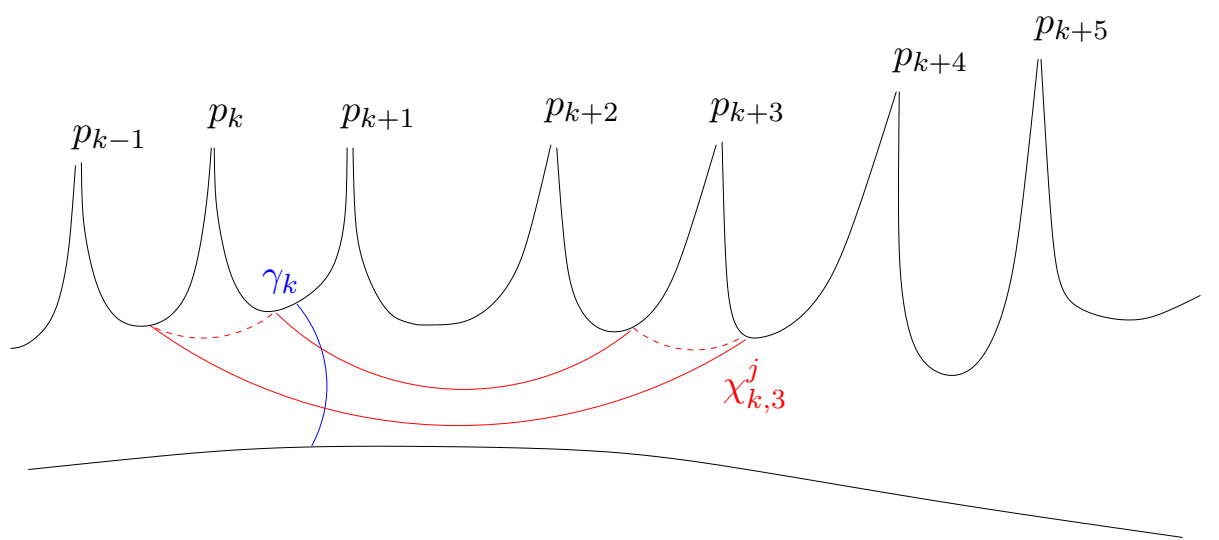

Figura 3.24: Desenho da geodésica $\chi_{k, 3}^{j}$ em vermelho. Em azul a geodésica $\gamma_{k}^{j}$.

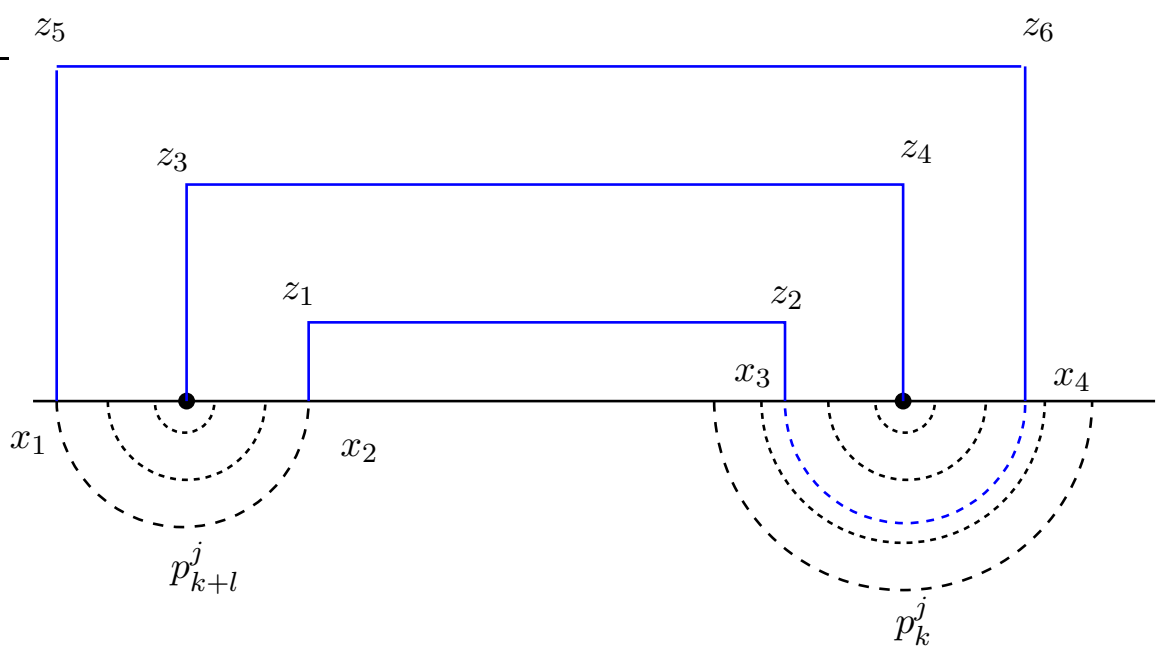

Figura 3.25: Desenho do anel A que separa $p_{k}^{j}$ e $p_{k+l}^{j}$ do resto dos furos

A fronteira exterior do anel $A$ é a curva fechada que pasa pelos pontos $x_{1} \sim_{\mathcal{P}} x_{2}, z_{1}, z_{2}, x_{3} \sim_{\mathcal{P}}$ $x_{4}, z_{6}, z_{5}$, enquanto que a fronteira interior do $A$ é os segmento poligonal que pasa por $p_{k+l}^{j}, z_{3}, z_{4}, p_{k}^{j}$. $\mathrm{O}$ anel $A$ é redutível a geodésica $\chi_{k, l}^{j}$ procurada. Usando a densidade Euclideana $\rho_{e}(z)|d z|$, tem-se $\mathcal{A}_{\rho_{e}}(A)=4 a_{k, l}^{2}+4 a_{k, l} \cdot t+2 a_{k, l} \cdot d_{P}\left(\hat{p}_{k}^{j}, \hat{p}_{k+l}^{j}\right)$. Como $\mathcal{L}_{\rho}(A)=a_{k, l}$, usando novamente o Lema 3.2.2,

$$
\begin{aligned}
\ell_{\infty}\left(\chi_{k, l}^{j}\right) & \leq \pi \cdot \frac{\mathcal{A}_{\rho_{e}}(A)}{\mathcal{L}_{\rho_{e}}(A)^{2}} \\
& =\pi \cdot \frac{4 a_{k, l}^{2}+4 a_{k, l} \cdot t+2 a_{k, l} \cdot d_{P}\left(\hat{p}_{k}^{j}, \hat{p}_{k+l}^{j}\right)}{a_{k, l}^{2}} \\
& =2 \pi\left(2+2 \frac{t}{a_{k, l}}+\frac{d_{P}\left(\hat{p}_{k}^{j}, \hat{p}_{k+l}^{j}\right)}{a_{k, l}}\right)
\end{aligned}
$$

Ao fazer $t \rightarrow 0$, conclui-se o seguinte resultado.

Proposição 3.5.4. Nas condições acima,

$$
\ell_{\infty}\left(\chi_{k, l}^{j}\right) \leq 4 \pi+2 \pi \cdot \frac{d_{P}\left(\hat{p}_{k}^{j}, \hat{p}_{k+l}^{j}\right)}{a_{k, l}}
$$


Exemplo 3.5.5. Considere-se a esfera furada do exemplo 3.3.4. Fixado um superíndice $j=1,2,3,4$, e dado qualquer $k \geq 1$, é fácil ver que $d_{P}\left(\hat{p}_{k}^{j}, \hat{p}_{k+1}^{j}\right)=a^{k}+a^{k+1}+b^{k}$ e que $a_{k, l}=a^{k+1}$. Logo usando a proposição acima tem-se,

$$
\ell_{\infty}\left(\chi_{k, 1}^{j}\right) \leq 6 \pi+\frac{2 \pi}{a}\left(1+\left(\frac{b}{a}\right)^{k}\right)
$$

Observe-se que se $b \leq a$, então as curvas $\chi_{k, 1}^{j}$ têm comprimento uniformemente limitados por acima. Isto implica que existe $m>0$ tal que $m \leq \ell_{\infty}\left(\gamma_{k}^{j}\right)$ para todo $k \geq 0$ e $j=1,2,3,4$.

\subsection{A ferradura justa furada}

Seja $S_{\mathcal{P}}$ a esfera de papel associada à ferradura justa da seção 2.1. Chamaremos a este espaço também de ferradura justa e à correspondente esfera de papel furada $S_{\infty}$ de ferradura justa furada. Para finalizar a tese faremos um análisis das coordenadas de comprimento e as coordenadas de torção da ferradura justa furada. Antes de estimar tais coordenadas, alguns comentarios sobre a esfera de papel $S_{\mathcal{P}}$.

Lembre-se que $S_{\mathcal{P}}$ tem um único ponto irregular $q=\pi_{\mathcal{P}}(q)$ o qual é um $\infty$-vértice e ao mesmo tempo é ponto de acumulação do seus pontos cônicos $\left\{p_{n}\right\}_{n \in \mathbb{Z}^{*}}$, todos os quais são pontos 1-cônicos. É facil ver, usando o Teorema 2.2.11 que a estrutura complexa de $S_{\mathcal{P}} \backslash\{q\}$ extende-se ao ponto irregular $q$ com o qual, pelo Teorema de uniformização, existe um biholomorfismo $\varphi: S_{\mathcal{P}} \longrightarrow \hat{\mathbb{C}}$. Suponha que $\varphi(q)=\infty$ e denote por $w_{n}, n \neq 0$, as imagens $w_{n}:=\varphi\left(p_{n}\right)$. É importante destacar que não é conhecido uma expressão do biholomorfismo $\varphi$ nem da distribuição dos pontos $w_{n}, n \neq 0$, sobre o plano. Porém em [dCH12] prova-se a existencia de um módulo de continuidade para à aplicação $\varphi$, isto é, uma função $\phi:[0, \delta) \longrightarrow[0,+\infty)$ estritamente crescente com $\phi(0)=0$, que satisfaz o seguinte: Se $x_{0} \in S_{\mathcal{P}}$, então para todo $x \in B_{S_{\mathcal{P}}}\left(x_{0}, \delta\right)$,

$$
d_{\widehat{\mathbb{C}}}\left(\varphi(x), \varphi\left(x_{0}\right)\right) \leq \phi\left(d^{\mathcal{P}}\left(x, x_{0}\right)\right)
$$

A relação exata entre a distância esférica $d_{\mathbb{C}}$ e a distância $d^{\mathcal{P}}$ do espaço de papel aínda não é bem conhecida.

Por outro lado, seja $\hat{R}$ a reflexão em torno à diagonal $\Delta$ do quadrado $P$. Observe-se que $\hat{R}\left(\pi_{\mathcal{P}}^{-1}(q)\right)=\pi_{\mathcal{P}}^{-1}(q)$ e $\hat{R}\left(\pi_{\mathcal{P}}^{-1}(\mathcal{V})\right)=\pi_{\mathcal{P}}^{-1}(\mathcal{V})$, logo $\hat{R}$ pode ser $\pi_{\mathcal{P}}$-projetado numa aplicação continua $R: S_{\mathcal{P}} \longrightarrow S_{\mathcal{P}}$ a qual é de fato um homeomorfismo que reverte orientação cujo conjunto de pontos fixos consiste no círculo topológico $\Delta_{\mathcal{P}}:=\pi_{\mathcal{P}}(\Delta)$. Olhando as cartas locais que determinan a estrutura complexa de $S_{\mathcal{P}}$, é fácil verificar que $R$ é uma aplicação anti-holomorfa, logo a função $h: \hat{\mathbb{C}} \longrightarrow \hat{\mathbb{C}}$ dada por $h:=\varphi \circ R \circ \varphi^{-1}$ também é anti-holomorfa. Assim existem $a, b, c, d \in \mathbb{C}$, $a d-b c \neq 0$, tais que $h(z)=\frac{a \bar{z}+b}{c \bar{z}+d}$. Uma função anti-holomorfa na esfera de Riemann ou não tem pontos fixos, ou tem um ponto fixo, ou têm dois pontos fixos ou têm um círculo em $\mathbb{C}$ de pontos fixos, ver [Mas88], capítulo 1. No caso da função $h$ tem que ser a última possibilidade, com o qual $\varphi\left(\Delta_{\mathcal{P}}\right)$ é um círculo mesmo na esfera de Riemann e $h$ é a reflexão em torno a este círculo. Fazendo uma conjugação pode-se assumir que $\varphi\left(\Delta_{\mathcal{P}}\right)=\hat{\mathbb{R}}$, com o qual $h(z)=\bar{z}$, para todo $z \in \mathbb{C}$. Em consequência, como $R\left(p_{n}\right)=p_{-n}$ para todo $n \geq 1$, conclui-se que $w_{-n}=\bar{w}_{n}$. A figura 3.26 mostra uma possível distribuição das imagens $w_{n}, n \neq 0$, dos pontos 1-cônicos de $S_{\mathcal{P}}$ no plano $\mathbb{C}$; observe-se que se pode assumir que $\varphi\left(p_{1}\right)=w_{1}=i$. Possívelmente esta distribuição simétrica dos pontos $w_{n}$ sobre o plano complexo com relação a reta real pode contribuir de alguma forma no estudo de mais propriedades da ferradura justa e em particular, no conhecimento das características da função de uniformização $\varphi$ sobre a esfera furada $S_{\infty}$. 


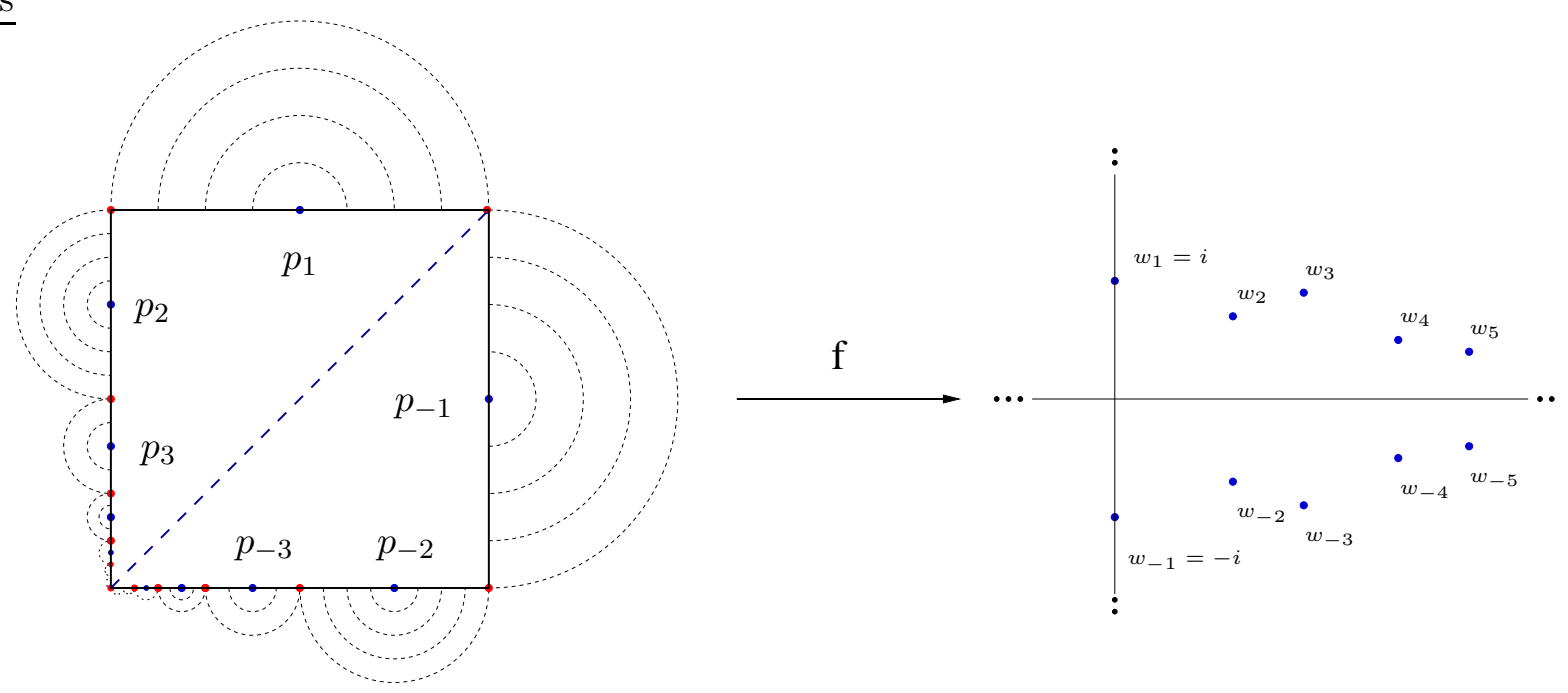

Figura 3.26: Possível distribuição dos pontos $w_{ \pm k}=\varphi\left(p_{ \pm k}\right)$

Por enquanto voltemos à ferradura justa furada $S_{\infty}$ e suas estimativas das coordenadas de comprimento. Dado que $S_{\mathcal{P}}$ tem somente um ponto irregular, a ferradura justa furada corresponde à uma flauta justa. Escolhemo a multicurva $\Gamma_{\infty}=\left\{\gamma_{k}\right\}_{k \geq 1}$ que separa em calças a $S_{\infty}$ como foi explicado na seção 3.1, de maneira que, para todo $k \geq 1$,

i) $\gamma_{2 k-1}$ separa os furos $\left\{p_{ \pm 1}, p_{ \pm 2}, \ldots, p_{ \pm k}\right\}$ de $\{q\} \cup\left\{p_{ \pm n}\right\}_{n>k}$.

ii) $\gamma_{2 k}$ separa os furos $\left\{p_{ \pm 1}, p_{ \pm 2}, \ldots, p_{ \pm k}, p_{k+1}\right\}$ de $\left\{q, p_{-k-1}\right\} \cup\left\{p_{ \pm n}\right\}_{n>k+1}$.

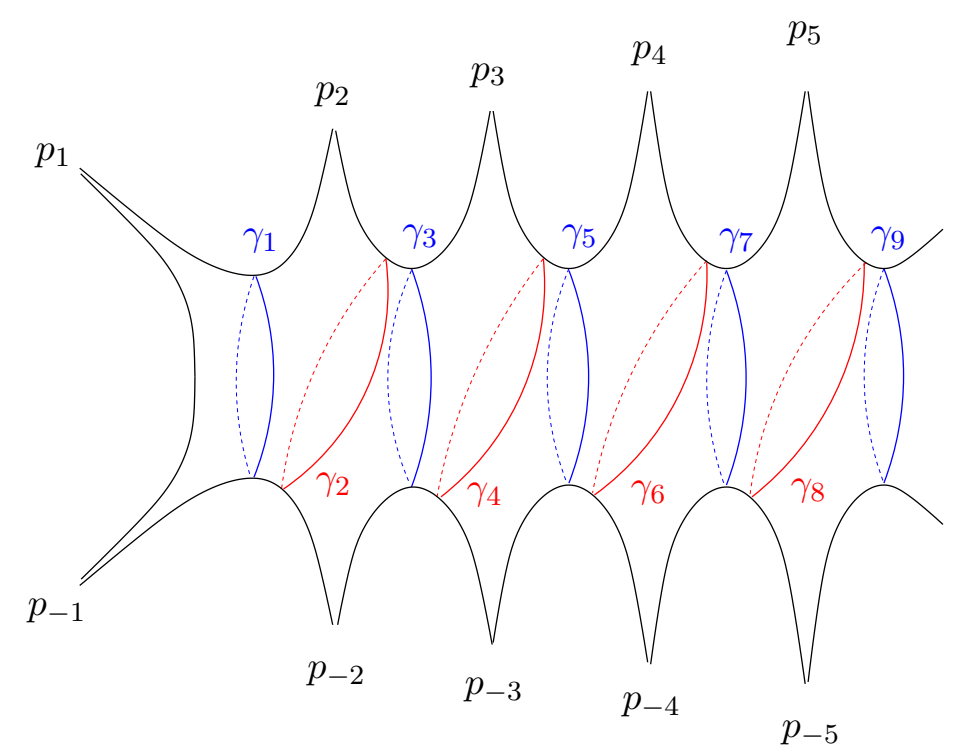

Figura 3.27: Ferradura justa furada e as curvas geodésicas $\gamma_{k}$.

A figura 3.27 mostra um desenho da ferradura justa furada com a decomposição em calças feita pela multicurva $\Gamma_{\infty}$. Usaremos o Teorema 3.3.1 para estimar por acima os comprimentos hiperbólicos das geodésicas $\gamma_{k}$. Para todo $k \geq 2$, é fácil ver que $m_{2 k-1}=\frac{4}{2^{k}}$ e $m_{2 k}=\frac{3}{2^{k}}$. As componentes de $\pi_{\mathcal{P}}^{-1}\left(D_{k}\right)$ são todas do tipo 1 com a exceção de um único componente que é do tipo 4 ; a pessar disso observe-se que para $k \geq 2, f_{2 k-1}=e_{2 k-1}=\frac{1}{2^{k}}$ e $f_{2 k}=e_{2 k}=\frac{1}{2^{k+1}}$. Portanto, 


$$
\ell_{\infty}\left(\gamma_{2 k-1}\right) \leq 4 \pi, \quad \ell_{\infty}\left(\gamma_{2 k}\right) \leq 6 \pi
$$

Observe-se que se $\gamma \subset S_{\infty}$ é uma curva homotópica à $\gamma_{2 k-1}$, a curva $R(\gamma)$ também é homotópica a $\gamma_{2 k-1}$. Isto implica em particular que $R\left(\gamma_{2 k-1}\right)=\gamma_{2 k-1}$, com o qual a imagem pelo $\varphi$ de esta curva deve ser simétrica à reta real $\mathbb{R}$ e intersectar-lhe ortogonalmente. Com respeito a curva $\gamma_{2 k}, R\left(\gamma_{2 k}\right)$ será outra geodésica em $S_{\infty}$ que separa os furos $\left\{p_{ \pm 1}, p_{ \pm 2}, \ldots, p_{ \pm k}, p_{-k-1}\right\}$ dos furos $\left\{q, p_{k+1}\right\} \cup\left\{p_{n}\right\}_{n>k+1}$.

Considere-se agora geodésicas $\beta_{k, l}, k \geq 2$ e $l \geq 1$, similares as curvas da seção 3.5, onde $\beta_{k, l}$ separa os furos $\left\{p_{k}, p_{k+1}, \ldots, p_{k+l}\right\}$ do resto dos furos. Seja $\beta_{-k+l}$ a geodésica fechada simples homotópica à $R\left(\beta_{k, l}\right)$; esta separa $\left\{p_{-k}, p_{-k-1}, \ldots, p_{-k-l}\right\}$ do resto dos furos. A figura 3.28 mostra um desenho de tais geodésicas. Pela Proposição 1.3.1 ii) e v), fixado $k \geq 2$, a família de geodésicas $\left\{\beta_{ \pm k+l}\right\}_{l \geq 1}$ são disjuntas duas a duas. Por outro lado $\gamma_{2 k-1}$ intersecta transversalmente a todas elas enquanto que $\gamma_{2 k}$ intersecta transversalmente a todas exceto a $\beta_{k, 1}$. Usando o argumento da seção 3.5, a Proposição 3.5.1 e o exemplo 3.5.2, tem-se,

$$
\ell_{\infty}\left(\beta_{ \pm k+l}\right) \leq 2^{l+2} \cdot \pi,
$$

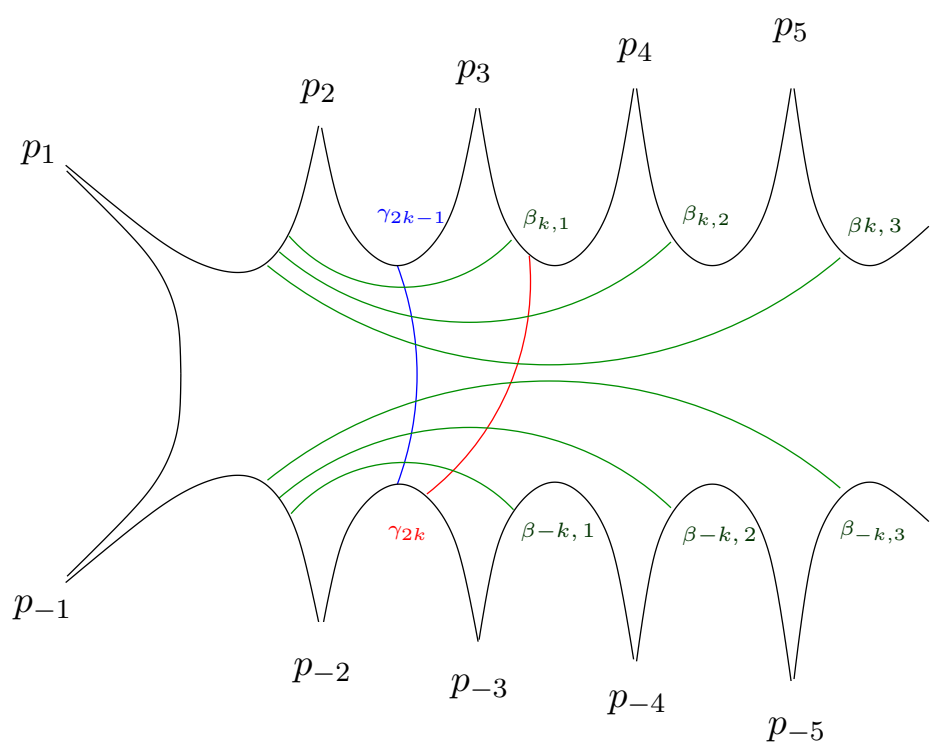

Figura 3.28: Geodésicas $\beta_{ \pm k, l}$, para $l=1,2,3$ e $k=2$.

com o qual,

$$
\begin{gathered}
\ell_{\infty}\left(\gamma_{2 k-1}\right) \geq 8 \sum_{l \geq 1} \eta\left(2^{l+2} \pi\right)=8 \sum_{l \geq 1} \log \operatorname{coth}\left(2^{l} \pi\right) \approx 1,4 \times 10^{-5} \\
\ell_{\infty}\left(\gamma_{2 k}\right) \geq 4 \eta(8 \pi)+8 \sum_{\geq 2} \eta\left(2^{l+2} \pi\right)=4 \log \operatorname{coth}(2 \pi)+8 \sum_{l \geq 2} \log \operatorname{coth}\left(2^{l} \pi\right)
\end{gathered}
$$

Em qualquer caso existem constantes $m, M>0$ tais que para todo $k \geq 1$,

$$
m \leq \ell_{\infty}\left(\gamma_{k}\right) \leq M
$$

Assim, o modelo Fuchsiano $G_{\infty}$ de $S_{\infty}$ é um grupo Fuchsiano do primeiro tipo e as métricas $d_{\mathcal{T}}$ e $d_{\mathcal{L}}$ do espaço de Teichmüller quaseconforme $\mathcal{T}\left(S_{\infty}\right)$ são topológicamente equivalentes. Por outro lado, se $\Sigma\left(S_{\infty}\right)$ denota a coleção de todas as geodésicas fechadas em $S_{\infty}$, cada elemento de $\Sigma\left(S_{\infty}\right)$ 
diferente de $\gamma_{k}$, deve intersectar transversalmente à alguma das curvas $\gamma_{k}$ com o qual conclui-se também que $\inf _{\gamma \in \Sigma\left(S_{\infty}\right)} \ell_{\infty}(\gamma)>0$.

Com relação as coordenadas de torção $\tau=\left(\tau_{k}\right)_{k \geq 1}$ da flauta $S_{\infty}$, mostraremos que existe $T>0$ tal que $\left|\tau_{k}\right| \leq T$ para todo $k \geq 1$. Observe-se que ao considerar os núcleos de Nielsen de duas calças consecutivas de $S_{\infty}$ e colar elas, tem-se uma superfície $S_{k}$ do tipo $(0,2,2)$ como as mostradas na figura 3.29. Assim que é suficiente estimar por acima os comprimentos hiperbólico das curvas geodésicas $\alpha_{k} \subset S_{k}$ que separam dois furos consecutivos do resto dos furos de $S_{\infty}$, para logo usar a fórmula de Okai na Proposição 1.3.10 no caso onde $\ell_{3}=\ell_{4}=0$, e assim conseguir estimativas para as coordenadas $\tau_{k}, k \geq 1$.
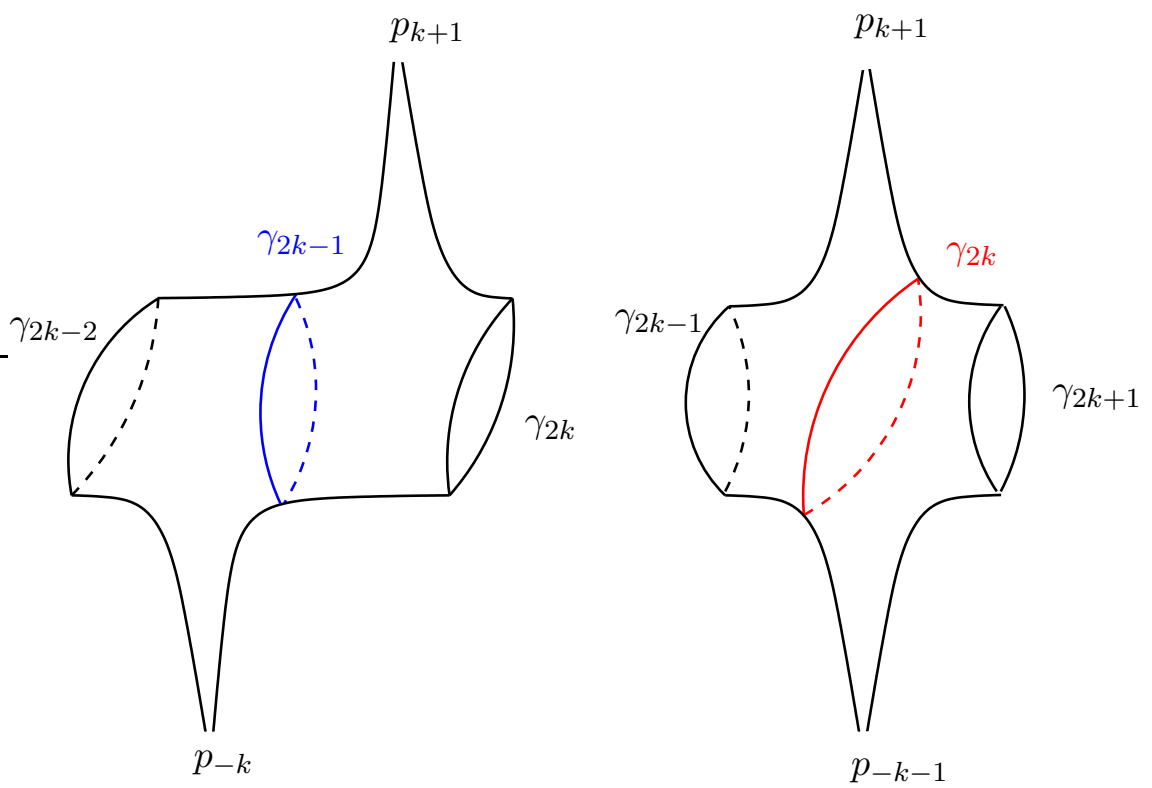

Figura 3.29: Duas calças consecutivas de $S_{\infty}$ coladas. A primeira tem como geodésica central à $\gamma_{2 k-1}$ e a segunda à $\gamma_{2 k}$. As geodésicas $\alpha_{2 k-1}$ e $\alpha_{2 k}$ separan os furos do bordo em cada superfície.

Observe-se que $\alpha_{2 k-1}$ limita um disco que contém os furos $p_{-k}$ e $p_{k+1}$ enquanto que $\alpha_{2 k}$ limita um disco que contém os furos $p_{k+1}$ e $p_{-k-1}$. Os aneis $A_{2 k-1}$ e $A_{2 k}$ mostrados na figura 3.30 são redutívels as curvas $\alpha_{2 k-1}$ e $\alpha_{2 k}$ respectivamente. Usando o Lema 3.2.2 com estos aneis e a densidade $\rho_{e}(z)|d z|$, tem-se,

$$
\ell_{\infty}\left(\alpha_{2 k-1}\right) \leq 18 \pi, \quad \ell_{\infty}\left(\alpha_{2 k}\right) \leq 12 \pi
$$

Igualmente, como as curvas $\alpha_{k}$ intersectan as curvas $\gamma_{k}$ para todo $k \geq 2$, e estas têm comprimentos limitados superiormente por uma constante, então as curvas $\alpha_{k}$ também são limitados inferiormente por uma constante. Assim a familia $\left\{\ell_{\infty}\left(\alpha_{k}\right)\right\}_{k \geq 1}$ está limitada por constantes positivas. Segue-se da fórmula de Okai, que a sequência $\tau=\left(\tau_{k}\right)_{k \geq 1}$ deve estar limitada.

Em particular, observe-se que as geodésicas $\alpha_{2 k}$ são transformados pela reflexão $R$ numa curva homotópica em $S_{\infty}$ a elas mesmas. Portanto elas deven ser invariantes pela reflexão $R$ e assim, as geodésicas $\alpha_{2 k}$ devem ser simétrica com respeito a curva $\Delta_{\mathcal{P}} \backslash\{q\}$. Por outro lado, sendo as curvas $\left\{\alpha_{2 k}\right\}_{k}$ com comprimento limitado superiormente, tal vez pode-se provar que existe $M>0$ tal que $\left|\operatorname{Im} w_{ \pm k}\right| \leq M$, para todo $k \geq 1$.

Para finalizar, a ferradura justa pertence a uma família infinita $\left\{f_{i}\right\}_{i \in I}$ de homeomorfismos pseudo-Anosov generalizados chamada de família unimodal (ver [dCH04] para mais detalhes), onde cada $f_{i}$ esta definido sobre uma esfera de papel $S_{i}$ que pode ou não ter pontos irregulares; aquí o conjunto de índices $I$ é um subconjunto enumerável e denso do intervalo $(\sqrt{2}, 2]$ e $f_{2}$ corresponde 

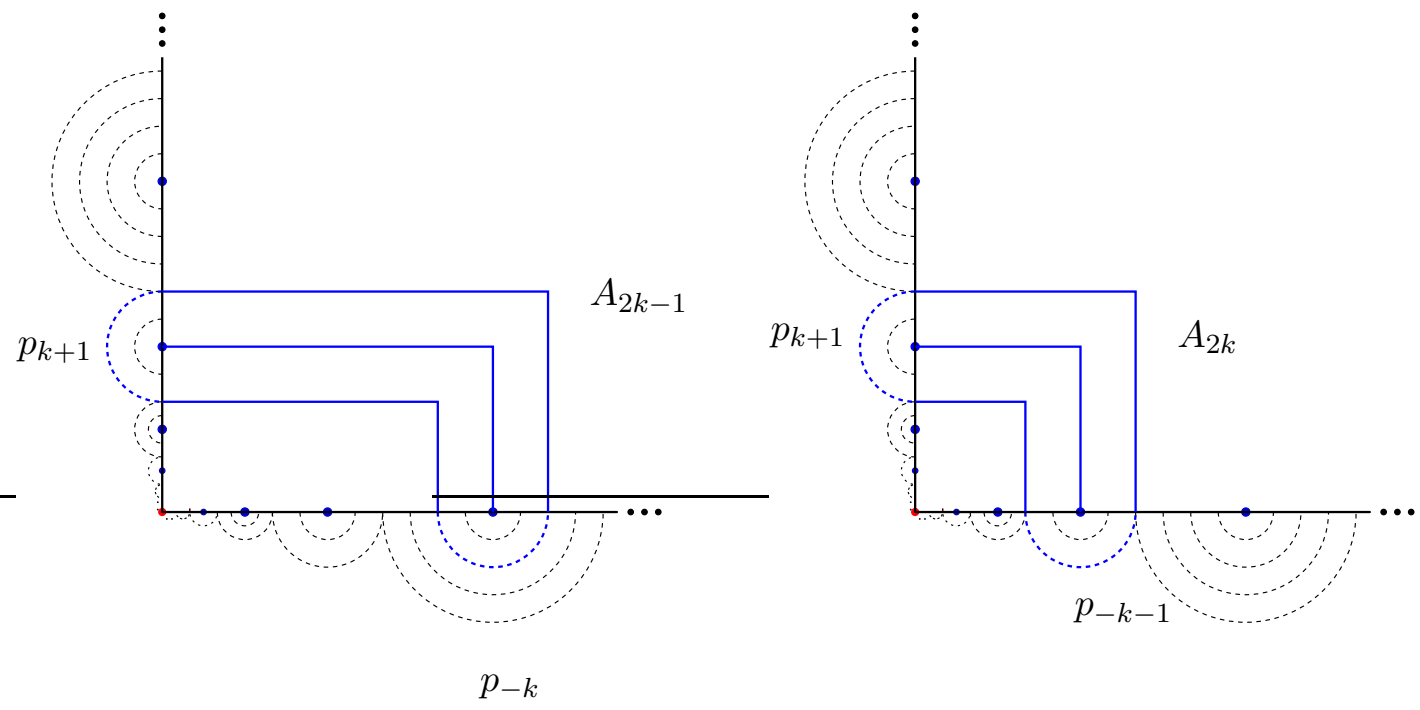

Figura 3.30: Aneis $A_{2 k-1}$ na esquerda, e $A_{2 k}$ na direita, na ferradura justa furada

exatamente com a ferradura justa. Quando o espaço de papel $S_{i}$ tem irregularidades, estos correspondem aos pontos de acumulação das singularidades das folheações mensuradas invariantes pela aplicação $f_{i}$ e localmente os pareamentos perto de uma irregularidade são como os pareamento da ferradura justa ou como os pareamento do exemplo 3.3.4, onde todos eles têm comprimentos proporcionais a potencias $\lambda^{n}$, sendo $\lambda>1$ a constante de expansividade na folheação inestável do homeomorfismo $f_{i}$. Usando as técnicas desenvolvidas nesta tese, principalmente o Teorema $3.3 .2 \mathrm{e}$ a Proposição 3.5.4, não deve ser difícil demostrar o seguinte resultado.

Teorema 3.6.1. Se $S_{\infty}$ é a esfera de papel furada associada a uma esfera de papel da família unimodal com pontos irregulares, então existem constantes $m, M>0$ tais que, para cada geodésica $\gamma$ na multicurva $\Gamma_{\infty}$ que decompoe à $S_{\infty}$ em calças como foi descrito na seção 3.1, tem-se,

$$
m \leq \ell_{\infty}(\gamma) \leq M
$$




\section{Referências Bibliográficas}

[Ahl73] L.V. Ahlfors. Conformals invariants: Topics in geometric function theory. McGraw-Hill, New York, 1973. 21

$\left[\right.$ ALP $\left.^{+} 11\right]$ D. Alessandrini, L. Liu, A. Papadopoulos, W. Su e Z. Sun. On Fenchel-Nielsen coordinates on Teichmüller spaces of surfaces of infinite type. Ann. Acad. Sci. Fenn. Math., 36, no. 2:621-659, 2011. 3, 25

[ALPS12a] Daniele Alessandrini, Lixin Liu, Athanase Papadopoulos e Weixu Su. On local comparison between various metrics on Teichmüller spaces. Geometriae Dedicata, 157(1):91110, 2012. 3

[ALPS12b] Daniele Alessandrini, Lixin Liu, Athanase Papadopoulos e Weixu Su. On various Teichmüller spaces of a surface of infinite topological type. Proceedings of the American Mathematical Society, 140(2):561-574, 2012. 3, 25

[ALPS16] Daniele Alessandrini, Lixin Liu, Athanase Papadopoulos e Weixu Su. On the inclusion of the quasiconformal Teichmüller space into the length-spectrum Teichmüller space. Monatshefte für Mathematik, 179(2):165-189, 2016. 3, 25

[APLS11] Daniele Alessandrini, Athanase Papadopoulos, Lixin Liu e Weixu Su. The behaviour of Fenchel-Nielsen distance under a change of pants decomposition. arXiv preprint arXiv:1105.0202, 2011. 3, 23

[Bas90] A. Basmajian. Constructing pairs of pants. Annales Academiae Scientiarum Fennicae. Series A I. Mathematica, 15, 1990. 27

[Bas93] A. Basmajian. Hyperbolic structure for surfaces of infinite type. American Math Soc, $336,1993.3,5,11,12,18,26,27,30$

[Bea83] A. Beardon. The geometry of discrete groups. Springer-Verlag, New York, 1983. 13, 20

[Ber12] M. Vinhas Bertolini. Espaços de papel. Dissertação de Mestrado, IME, São Paulo University, 2012. 3, 33, 38, 43

[Ber16] M. Vinhas Bertolini. Groups representations and Real Trees. Tese de Doutorado, IME, São Paulo University, 2016. 4

[BH95] M. Bestvina e M. Handel. Train-tracks for surface homeomorphisms. Topology, 34(1):109-140, 1995. 1

[BK08] Ara Basmajian e Youngju Kim. Geometrically infinite surfaces with discrete length spectra. Geometriae Dedicata, 137(1):219, 2008. 27

[Bon09] F. Bonahon. Low dimensional geometry. AMS, Providence, 2009. 5

[BŠ17] Ara Basmajian e Dragomir Šarić. Geodesically complete hyperbolic structures. Em Mathematical Proceedings of the Cambridge Philosophical Society, páginas 1-24. Cambridge University Press, 2017. 3, 27 
[Bus92] P. Buser. Geometry and spectra of compact Riemann surfaces. Progress in mathematics, Brikhauser, Boston Inc, Boston MA, 1992. 5, 20

[CGL06] R. Chamanara, F. P. Gardiner e N. Lakic. A hyperelliptic realization of the horseshoe and baker maps. Ergodic Theory and Dynamical Systems, 26(6):1749-1768, 2006. 33, 41

[Cha04] R. Chamanara. Affine automorphism groups of surfaces of inifinite type. In the tradition of Ahlfors and Bers, III, 355, 2004. 40

[Com11] Mark Comerford. Short separating geodesics for multiply connected domains. Central European Journal of Mathematics, 9(5):984-996, 2011. 22

[dC05] Andre de Carvalho. Extensions, quotients and generalized pseudo-Anosov maps. Graphs and patterns in mathematics and theoretical physics, 73:315-338, 2005. iii, v, 1, 3, 33, 35,36

[dCH04] André de Carvalho e Toby Hall. Unimodal generalized pseudo-Anosov maps. Geometry E Topology, 8(3):1127-1188, 2004. 36, 87

[dCH10] André de Carvalho e Toby Hall. Paper surfaces and dynamical limits. Proceedings of the National Academy of Sciences, 107(32):14030-14035, 08 2010. 1, 2, 3, 36

[dCH12] André de Carvalho e Toby Hall. Paper folding, Riemann surfaces and convergence of pseudo-Anosov sequences. Geometry $\& 3$ Topology, 16(4):1881-1966, 2012. 3, 33, 38, 41, $43,44,45,46,47,84$

[dCH13] André de Carvalho e Toby Hall. Riemann surfaces out of paper. Proceedings of the London Mathematical Society, 108(3):541-574, 2013. 3, 43

[Evr17] Özgür Evren. Inequivalent topologies on the Teichmüller space of the flute surface. Proceedings of the American Mathematical Society, 145(6):2607-2621, 2017. 3, 27

[FM11] Benson Farb e Dan Margalit. A primer on mapping class groups (pms-49). Princeton University Press, 2011. 5, 20

[Haa96] Andrew Haas. Dirichlet points, Garnett points, and infinite ends of hyperbolic surfaces. i. Annales Academiae Scientiarum Fennicae. Series A I. Mathematica, 21(1):3-29, 1996. 27

[Hem] Joachim A. Hempel. On the uniformization of the n-punctured sphere. Bulletin of the London Mathematical Society, 20(2):97-115. 2

[HS07] Andrew Haas e Perry Susskind. The geometry at infinity of a hyperbolic Riemann surface of infinite type. Geometriae Dedicata, 130(1):1-24, Dec 2007. 27, 31, 32

[Iva01] D. Burago, Y. Burago, S. Ivanov. A course in metric geometry. American mathematical society, providence,RI, 2001. 5, 6, 8, 9

[J.H06] J.H.Hubbard. Teichmüller theory and applications of geometry, topology, and dynamics:Teichmüller theory. Matrix editions, 2006. 4, 5, 14, 19, 20, 22

[Kat88] Svetlana Katok. Fucshian group. Chicago Press, 1988. 5, 13

[Kee74] Linda Keen. Collars on riemann surfaces. Em Discontinuous groups and Riemann surfaces (Proc. Conf., Univ. Maryland, College Park, Md., 1973), páginas 263-268, 1974. 21 
[Kin11] Erina Kinjo. On Teichmüller metric and the length spectrums of topologically infinite Riemann surfaces. Kodai Math. J., 34(2):179-190, 06 2011. 3, 25

[Lix99] Liu Lixin. On the length spectrums of non-compact Riemann surfaces. Ann. Acad. Sci. Fenn. Math., 24(1):11-22, 1999. 25

[LSW08] Lixin Liu, Zongliang Sun e Hanbai Wei. Topological equivalence of metrics in Teichmüller space. Em ANNALES-ACADEMIAE SCIENTIARUM FENNICAE MATHEMATICA, volume 33, página 159. ACADEMIA SCIENTIARUM FENNICA, 2008. 25

[Mas88] Bernard Maskit. Kleinian group. Springer-Verlag, New York, 1988. 5, 14, 15, 84

[Moo25] R.L Moore. Concerning upper semi-continuous collections of continua. Trans. Amer. Math. Soc, 27:416-428, 1925. 8

[Oka93] T. Okai. Effects of change of pants decompositions on their Fenchel-Nielsen coordinates. Kobe J. Math, 10:215-223, 1993. 23

[Ota96] Jean-Pierre Otal. Le théorème d'hyperbolisation pour les variétés fibrées de dimension 3. Astérisque, 1996. 4

[PRT $\left.{ }^{+} 11\right]$ Ana Portilla, José M Rodríguez, Eva Tourís et al. A real variable characterization of Gromov hyperbolicity of flute surfaces. Osaka Journal of Mathematics, 48(1):179-207, 2011. 27

[Ran16] Anja Randecker. Geometry and topology of wild translation surfaces. KIT Scientific Publishing, 2016. 1, 41

[Sar15] D. Saric. Fenchel-Nielsen coordinates for asymptotically conformal deformations. ArXiv e-prints, Julho 2015. 3

[Shi03] H. Shiga. On a distance defined by the length sprectrum on Teichmüller space. Annales Academiae Scientiarum Fennicae. Series A I. Mathematica, 28:315-326, 2003. 3, 25

[Why63] G. T. Whyburn. Analytic topology. American mathematical society, providence,RI, 1963. 8,9

[YT92] Imayoshi Y e M. Taniguchi. An introduction to Teichmüller spaces. Springer-Verlag, Tokio, 1992. 5, 22, 30

[Zor06] Anton Zorich. Flat surfaces. Frontiers in number theory, physics, and geometry I, páginas 439-585, 2006. 1, 40

[ZT88] P G Zograf e L A Takhtadzhyan. On Liouville's equation, accessory parameters, and the geometry of Teichmüller space for Riemann surfaces of genus 0. Mathematics of the USSR-Sbornik, 60(1):143, 1988. 2 